\title{
Contributions of Local Farming to Urban Sustainability in the Northeast United States
}

\author{
Goldstein, Benjamin Paul; Hauschild, Michael Zwicky; Fernandez, John E.; Birkved, Morten
}

\section{Published in:}

Environmental Science and Technology

Link to article, DOI:

10.1021/acs.est.7b01011

Publication date:

2017

Document Version

Peer reviewed version

Link back to DTU Orbit

Citation (APA):

Goldstein, B. P., Hauschild, M. Z., Fernandez, J. E., \& Birkved, M. (2017). Contributions of Local Farming to Urban Sustainability in the Northeast United States. Environmental Science and Technology, 51(13), 7340-7349. https://doi.org/10.1021/acs.est.7b01011

\section{General rights}

Copyright and moral rights for the publications made accessible in the public portal are retained by the authors and/or other copyright owners and it is a condition of accessing publications that users recognise and abide by the legal requirements associated with these rights.

- Users may download and print one copy of any publication from the public portal for the purpose of private study or research.

- You may not further distribute the material or use it for any profit-making activity or commercial gain

- You may freely distribute the URL identifying the publication in the public portal 


\section{Contributions of local farming to urban}

\section{2 sustainability in the Northeast United States}

3 Benjamin P. Goldstein ${ }^{* \dagger}$, Michael Z. Hauschild , John E. Fernández $z^{\dagger}$, Morten Birkved

$4{ }^{\dagger}$ Technical University of Denmark, Quantitative Sustainability Assessment Division,

5 Produktionstorvet, Building 424, Kongens Lyngby, 2800, Denmark

6 Massachusetts Institute of Technology, Department of Architecture, 77 Massachusetts Avenue,

7 5-419, Cambridge, 02139, United States

$8 \quad *$ Corresponding author: Phone: +45 45254561; fax: +45 45933435; e-mail: bgol@dtu.dk 


\section{Abstract}

10 Food consumption is an important contributor to a city's environmental impacts (carbon 11 emissions, land occupation, water use, etc.) Urban farming (UF) has been advocated as a means

12 to increase urban sustainability by reducing food-related transport and tapping into local

13 resources. Taking Boston as an illustrative Northeast US city, we developed a novel method to

14 estimate sub-urban, food-borne carbon and land footprints using multi-region-input-output

15 modeling and nutritional surveys. Computer simulations utilizing primary data explored UF's

16 ability to reduce these footprints using select farming technologies, building on previous city-

17 scale UF assessments which have hitherto been dependent on proxy data for UF. We found that

18 UF generated meagre food-related carbon footprint reductions (1.1-2.9\% of baseline $2211 \mathrm{~kg}$

$19 \mathrm{CO}_{2}$ equivalents/capita/annum) and land occupation increases $\left(<1 \%\right.$ of baseline $9000 \mathrm{~m}^{2}$ land

20 occupation/capita/annum) under optimal production scenarios, informing evidence-based urban

21 design in the region. Notwithstanding UF's marginal environmental gains, UF could help Boston

22 meet national nutritional guidelines for vegetable intake, generate an estimated \$160 million US

23 in revenue to growers and act as a pedagogical and community building tool, though these

24 benefits would hinge on large-scale UF proliferation, likely undergirded by environmental 25 remediation of marginal lands in the city. 


\section{Introduction}

Food consumption is a key driver of a city's environmental burdens ${ }^{1,2}$, and in the United

29 States (US) per capita impacts are amongst the highest globally ${ }^{3,4}$. Many cities in the Northeast

30 US are promoting urban farming (UF) - food production within the city, allowing for material

31 and energy exchange between city and farm ${ }^{5}$ - as a joint environmental and social sustainability

32 exercise $e^{6,7}$. Up to $20 \%$ of global food supply already comes from within cities, primarily in the

33 Global South ${ }^{8,9}$, but the potential in the Global North's cities to produce their own food on the

34 ground and buildings is believed to be substantial ${ }^{10-12}$. Hypothetical assessments of UF at the

35 city-scale have demonstrated reduced food related GHG emissions ${ }^{13,14}$ and land occupation ${ }^{14}$,

36 giving the impression that pro-UF policies can contribute to more sustainable urban food supply

37 networks. Despite UF's perceived environmental benefits, the recent spurt of pro-UF actions by

38 the cities of the Northeast US that include codification in land use laws ${ }^{7,15}$ and multi-city

39 commitments to increased local food production ${ }^{6}$ require deeper reflection about their systemic

40 environmental implications.

UF advocates tend to focus on the distance from farm to fork, equating local food with

42 environmentally sustainable food ${ }^{16,17}$, oversimplifying the complexity of food sustainability to a

43 single aspect. Reducing distribution burdens and wastage by co-locating food production and

44 consumption can lead to environmentally leaner production networks ${ }^{18,19}$, but contrasting results

45 have been found when large energy inputs are needed for space heating and lighting ${ }^{20,21}$. UF in

46 the Northeast US has demonstrated lower embodied greenhouse gas (GHG) emissions compared

47 to conventional supply networks in some instances, but with tradeoffs in other indicators (land

48 occupation, water scarcity) and potentially significant burdens from farm capital ${ }^{21}$. UF studies at

49 neighborhood and city scales have estimated reductions in food-borne GHG emissions ${ }^{13,14}$ and 
50 land occupation ${ }^{14}$, although these findings are not transferable to the US Northeast due to

51 climatic differences. The use of data for conventional agricultural production (minus transport

52 and wastage) as a proxy for UF production due to data gaps ${ }^{13,14}$ biased the assessments in UF's

53 favor.

This article provides a level of analysis that has been absent in previous UF sustainability

55 work. We used primary data from multiple urban farms in the US Northeast to evaluate the

56 environmental tradeoffs of substituting UF for conventional produce at the city-scale in this

57 region (assessing strictly horticultural products), including interactions with the host city's

58 material and energy systems. Multi-region input-output based environmental life cycle

59 assessment (MRIO-LCA) was combined with nutritional surveys to model baseline food-borne

60 environmental burdens at sub-urban granularity, in contrast to earlier work that has assumed

61 equivalency between per capita city and national food intakes. Potential nutritional and economic

62 benefits of UF were also considered.

63 Boston, US was used as a representative case city for the Northeast US. Though denser

64 than many cities in the region, Boston's monocentric layout typifies most Northeast US cities,

65 particularly in the densely populated Northeast Megalopolis. Importantly, Boston's climate

66 mirrors that of the Northeast US, with an outdoor growing season roughly from April through

67 October, and cold winters necessitating indoor growing reliant on external heating from the

68 region's predominantly fossil-fuel driven grid.

69 Methods

70 Two overarching tasks were performed here: estimating baseline environmental impacts

71 from Boston's food demands and modeling UF in Boston.

\section{Baseline environmental performance}


EXIOBASE v2.3 MRIO model was applied to estimate Boston's food related

74 environmental burdens in 2010. EXIOBASE is a trade-linked model accounting for global

75 economic activity in 2007, detailing domestic production, bilateral trade and final consumption

76 of 43 regions accounting for $\sim 90 \%$ of global GDP ${ }^{22}$. MRIO-LCA has been described in detail

77 elsewhere ${ }^{23,24}$, but the method's core are environmental extensions coupling production activities

78 to resource and pollution intensities per unit economic output, facilitating the allocation of

79 environmental impacts and resource draws to end consumers. Such top-down analysis is suitable

80 for consumption based environmental accounting of large systems, having been applied at the

81 national ${ }^{25-27}$ and urban scales ${ }^{28,29}$. We chose EXIOBASE due to the high level of disaggregation

82 (200 products), including pertinent food items.

83 The assessed indicators were land use and global warming potential (GWP). Land use

84 accounts for crop, pasture and forest land occupation in $\mathrm{m}^{2}$. The GWP extension includes $\mathrm{CO}_{2}$,

$85 \mathrm{CH}_{4}, \mathrm{~N}_{2} \mathrm{O}$ and $\mathrm{SF}_{6}$ emissions, employing IPCC 2013 methodology to convert emissions to the

86 radiative forcing in equivalent mass $\mathrm{CO}_{2}$ over a 100 year time horizon $\left(\mathrm{kg} \mathrm{CO}_{2} \mathrm{e}\right)$.

87 Input-output models take the product of national footprint multipliers (e.g. $\mathrm{kg} \mathrm{CO}_{2} \mathrm{e} / \$$

88 final demand product) and final consumption (\$ final demand for product) to estimate demand-

89 side footprints, insinuating that doubling food expenditures doubles food consumption and

90 environmental stress. Whilst there is a correlation between income and food related

91 environmental burdens at the national scale, it appears to follow a logarithmic trend, hinting at an

92 income level beyond which food intake and environmental impacts plateau ${ }^{1,3}$. For a wealthy

93 nation such as the US with a low Engel's ratio ${ }^{30}$ (food expenditures as a percentage of total

94 income), assuming a linear relationship between food expenditures and consumption is

95 erroneous. US nutritional surveys show slight differences between the food consumption of high 
96 and low income residents, most notably for environmentally intensive foods (less than $10 \%$

97 difference between the groups for per capita meat and dairy intake by mass) $)^{31}$, despite markedly

98 elevated food expenditures by wealthy Americans ${ }^{32}$. Lastly, the higher prevalence of obesity in

99 poorer Americans highlights the incongruences between food expenditures and intake ${ }^{33}$.

100 We circumnavigated this challenge using a top down approach, ascribing total GWP and

101 land use from US final food consumption to total available calories in the US, akin to Jones and

102 colleagues ${ }^{28,29}$. Using a concordance matrix linking calorific availability for over 200 foods with

103 products in the EXIOBASE model (e.g. calories of grains with the EXIOBASE product

104 'Cereals'), embodied environmental intensity per calorie was estimated. Total calories available

105 for different foods were taken as the product of the 2007 US population and average US calorific

106 intake for the years 2007-2010 from the Center for Disease Control's National Health and

107 Nutrition Examination Survey (NHANES) ${ }^{34}$ corrected for supply chain losses using US

108 Department of Agriculture (USDA) loss adjusted availability data ${ }^{35}$. Tables S1 to S24 in the

109 supplementary information document this process.

110 Intakes of foods for US demographics based on sex and age group were taken from the

111 NHANES data for 2007-2010. Sex and age were chosen to develop population sub-groups as

112 these are both strong determinants of food volume consumed (males tend to eat more than

113 females at most ages) and dietary habits (e.g. dark green vegetable intake is nearly zero before

114 age 14 and then proceeds parabolically with age $)^{34}$. This sub-grouping also allowed for more

115 nuanced modelling than the low/high income binary afforded by the publically available

116 NHANES data relating income to food intake ${ }^{31}$. Demographics data for Boston at the block-

117 group level (geographies of population 600-3000) were taken from the 2010 US Census.

118 Combining census data, calorific intakes for different demographics and embodied GWP and 
119 land use per calorie delivered, food-borne environmental burdens for 560 block-groups in Boston

120 were calculated. Figure 1 outlines this workflow while the supporting information details the

121 data manipulation and calculations.

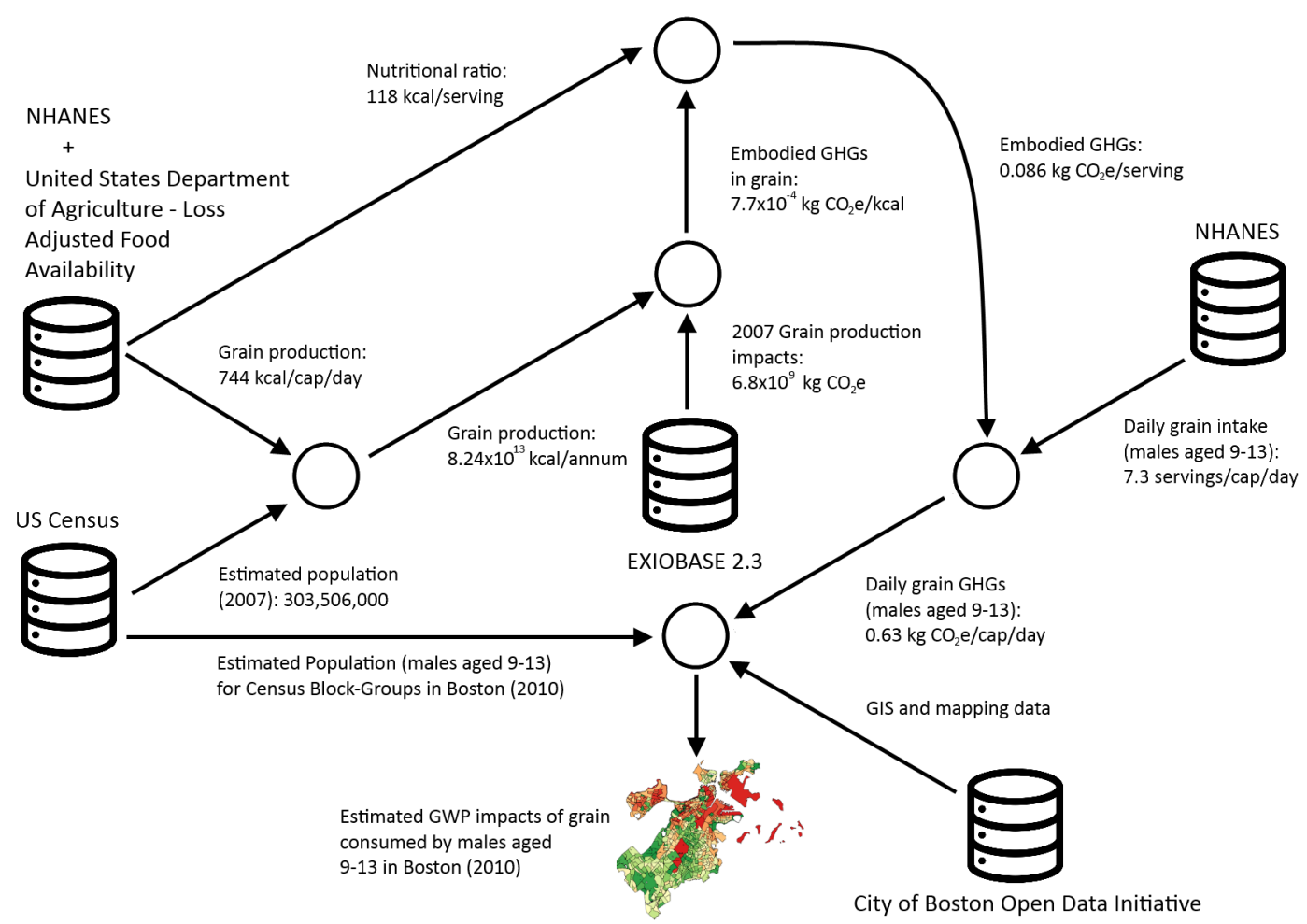

123 Figure 1. Workflow in generating baseline environmental performance, using grain consumption

124 by adolescent males as an example.

\section{Modelling UF in Boston}

126 We assessed two UF forms: empty-lot and rooftop farms, both open to the ambient

127 environment. Data were also collected on additional UF forms (ground/rooftop greenhouses and

128 automated precision agriculture), but were not included in the model since they displayed worse

129 environmental performance compared to conventional produce ${ }^{21}$, and therefore, were poor

130 candidates when assessing UF's substitution benefits. Resource use and yield data for two

131 empty-lot farms and one rooftop farm were collected over the 2015 growing season for 14

132 vegetables covering $32 \%$ by mass ( $44 \%$ excluding potatoes) and $24 \%$ by calories ( $50 \%$ 
133 excluding potatoes) of total average US vegetable consumption ${ }^{35}$. Although only some of the 14

134 vegetables were environmentally preferable to conventional when produced with UF (see

135 supporting information), including all vegetables was representative of actual production

136 scenarios where farmers are free to choose their crops.

137 GWP and land use impacts from UF production were modeled with process-LCA from

138 cradle to point of purchase, aligning with the EXIOBASE scope (transport impacts were added

139 manually to EXIOBASE results) and capturing the majority of food related impacts ${ }^{36-38}$.

140 Conventional crop GHG intensities were taken as mean values from Keolian and Heller's review

141 of food LCAs ${ }^{39}$, corrected for distribution losses and average transport distances. Land use was

142 taken as direct agricultural land occupation from USDA production data ${ }^{40}$, corrected for

143 distribution losses ${ }^{35}$, and excluded final distribution burdens. LCA modelling details are in the

144 supporting information.

145 Ground space potentially available for UF was determined using additive and subtractive

146 approaches. The additive approach assessed over 160,000 individual properties in Boston,

147 calculating total UF space as the sum of properties with land uses amenable to UF (vacant lots,

148 pasture, open land, cropland, transitional, etc.) The subtractive approach started with the city's

149 entire land area and subtracted land uses unsuitable for UF (steep slopes, impermeable surfaces,

150 protected parkland, etc.) to arrive at an upper estimate from the opposite direction. Rooftop UF

151 space was estimated using a dataset of 80,000 buildings in Boston. Lacking structural data, the

152 year of construction was used as a proxy for load bearing ability. We tested cutoff years from

1531900 to 2000 to quantify the effect of this choice on model results since this range covered $\sim 80 \%$

154 of the city's buildings. Buildings over 30 meters high, having sloped roofs or historically

155 protected were assumed unusable for UF. As some buildings lacked data on roof-type, 100-run 
156 Monte-Carlo simulations were performed for each cutoff year, examining the impact of

157 probabilistic roof-type assignment. The supporting information details the UF area estimates.

158 In considering UF interactions with the city we included avoided runoff, municipal

159 organic solid waste assimilation and building energy impacts. High and low estimates of runoff

160 reduction were taken as the average rainfall in Boston times the formally impermeable UF area,

161 using upper and lower retention values from previous studies ${ }^{41,42}$. Solid waste assimilative

162 capacity was taken from primary data on urban farm compost consumption converted to mass of

163 original organic waste. The same dataset used in calculating roof space includes heating and air

164 conditioning data which were combined with commercial ${ }^{43}$ and residential ${ }^{44}$ energy surveys to

165 estimate building energy loads. Previous studies of heating and cooling savings from vegetated

166 roofs were used to estimate energy savings from building situated $\mathrm{UF}^{45}$. UF interactions with

167 Boston's hydrological, waste and energy systems are detailed in the supporting information.

168 We modeled the most efficient application of Boston's UF space towards both land use

169 and GWP reduction. An algorithm was run whereby each block-group produced vegetables that

170 resulted in the largest reduction in GWP or land use depending on optimization goal, while

171 respecting local demands for each crop as a constraint. Space was allocated to a vegetable until

172 the block-group was satiated (at which point the next best vegetable for the optimization goal

173 was produced), UF space was exhausted or all vegetable needs were met. After all blocks-groups

174 had the chance to produce for themselves, those with extra capacity produced for those lacking

175 space until total vegetable needs for the city were met or Boston's UF space was exhausted. See

176 supplementary information for detailed explanation of optimization algorithm. 
178 were run. Within each scenario 10 different building age cutoffs were considered using 100-run

179 Monte-Carlo simulations. Table 1 outlines these scenarios.

\begin{tabular}{ll}
\hline Scenario & Description \\
\hline GWP(+) & Optimization for GWP reduction using additive \\
& method to estimate UF space \\
GWP(-) & Optimization for GWP reduction using subtractive \\
& method to estimate UF space \\
Land(+) & Optimization for land use reduction using additive \\
& method to estimate UF space \\
\hline Land(-) & Optimization for land use reduction using \\
& subtractive method to estimate UF space \\
\hline
\end{tabular}

$181 \quad$ Results and Discussion

182 Figure 2a shows the average, baseline GWP for Boston's food demands according to the

183 NHANES usual daily intakes for different demographics. Calculated GWP was $2211 \pm 55 \mathrm{~kg}$

$184 \mathrm{CO}_{2} \mathrm{e} / \mathrm{cap} / \mathrm{a}$ aligning with national assessments using EXIOBASE ${ }^{27}$, with the main impacts

185 emanating from the meat and dairy products (54\%). Figure $2 \mathrm{c}$ focuses on GWP impacts for the

186 individual block-groups which varied between 2078-2211 $\mathrm{kg} \mathrm{CO}_{2} \mathrm{e} / \mathrm{cap}$, where those with greater

187 proportions of adults and males sat at the upper end. The influence of meat and dairy agrees with

188 other assessments of the US diet ${ }^{29,38,39}$. GWP estimates are larger than process-LCA accounts of

189 the US $\operatorname{diet}^{39}$, but well aligned with other input-output analyses of US food consumption ${ }^{28,38}$, a

190 result of the latter method's enhanced value-chain coverage when building inventories. The tight

191 spread around Boston's mean and proximity of city and national averages support the use of the

192 latter as a proxy for urban level impacts, though caution is warranted when using this

193 simplification in settings with substantial income inequality. 
$\left.19510554 \mathrm{~m}^{2} / \mathrm{cap} / \mathrm{a}\right)$, agreeing with the earlier national EXIOBASE work ${ }^{27}$ and studies that have

196 pegged average US food-related land occupation between $0.86-1 \mathrm{ha} / \mathrm{a}^{46,47}$. Meat and dairy were

197 again dominant ( $50 \%)$, while fruits and vegetables were also key $(20 \%)$. The focus on animal

198 based products agrees with previous work, but the percentage of total land burdens is reduced.

199 Peters et al.'s assessments of US diets have found that animal products accounted for

200 approximately $75 \%$ of the $\sim 1$ ha/a land use burdens ${ }^{46,47}$, and Eshel and colleague's calculate

201 over $1 \mathrm{ha} / \mathrm{cap} / \mathrm{a}$ for animal-sourced foods alone ${ }^{48}$. Misalignment with these other studies might

202 stem from the calorie allocation method employed here, since EXIOBASE products divided

203 between animal- and vegetal-sourced foods (e.g. 'Food products nec' a catch-all EXIOBASE

204 product for various processed foods, accounting for $39 \%$ of total land use) are disproportionately

205 allotted to the latter, due to the poorer energetic returns per unit area when moving up trophic

206 levels ${ }^{47,48}$.

207
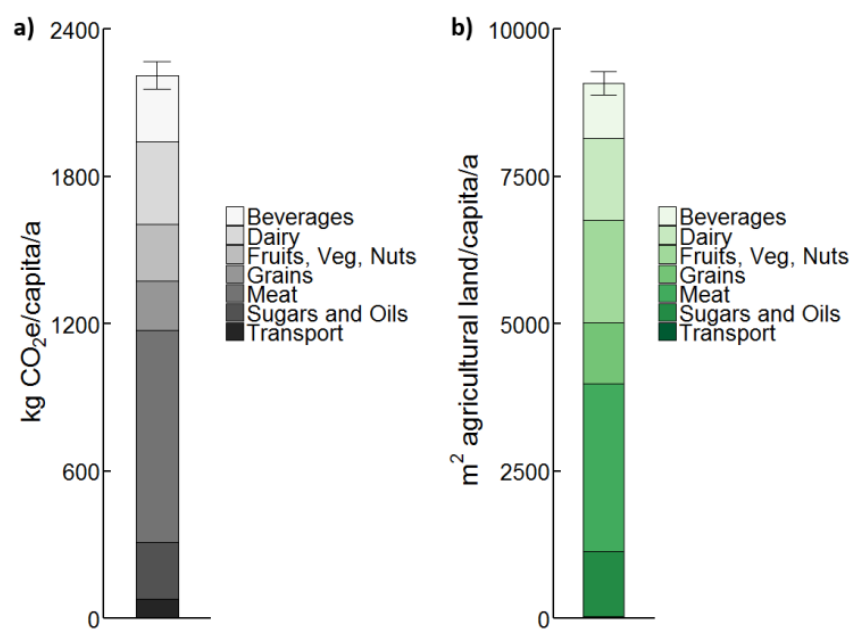

c)

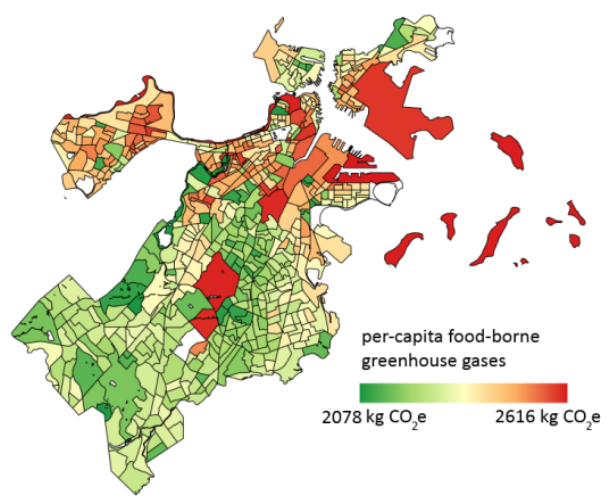

208 Figure 2a-c. Average baseline food related (A) GWP and (B) land use impacts for Boston in

2092010 based on NHANES demographic usual daily intakes. Error bars represent standard error 
210 amongst city population. (C) Average GWP at the block-group level, with uninhabited blocks

211 shown as white.

\section{Available UF space}

213 Figures 3a-b show Boston's available ground UF space calculated with subtractive and

214 additive methods estimated at 8846 and 2000 acres (28.8\% and 6.7\% Boston's area),

215 respectively. Naturally, the lower density block-groups with dispersed built forms tended to have

216 more UF potential, but appreciable area was also found in the former industrial areas and port

217 lands. These estimates ignored contaminated land that would likely be precluded from UF

218 without remediation, but are suitable approximations of where UF could be placed without

219 disturbing Boston's built form. Figure 3c-d presents 100-run Monte Carlo average UF available

220 roof area in each block-group for the lowest (1900) and highest (2000) construction cutoff years,

221 respectively. A 1900 cutoff resulted in 8828 available UF buildings with average area $195 \mathrm{~m}^{2}$

222 netting $424 \pm 8$ total acres. Using 2000 as a cutoff year left only 700 buildings with a mean area of

$223379 \mathrm{~m}^{2}$, covering a mere $26 \pm 3$ acres. The majority of Boston's buildings were built prior to 1920 ,

224 and accordingly, estimated rooftop UF space remained below 200 acres at cutoffs above this year

225 (see supplementary information figures for further details). 
a)

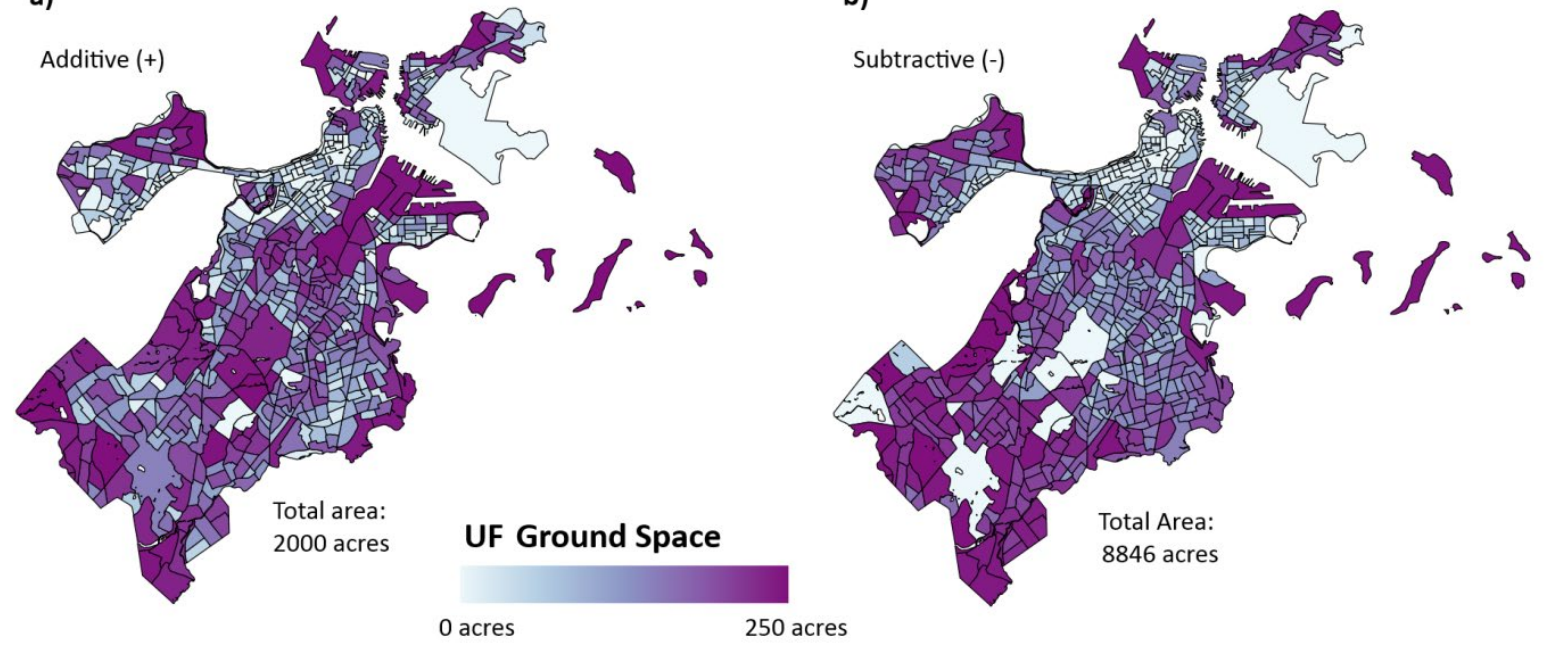

c)

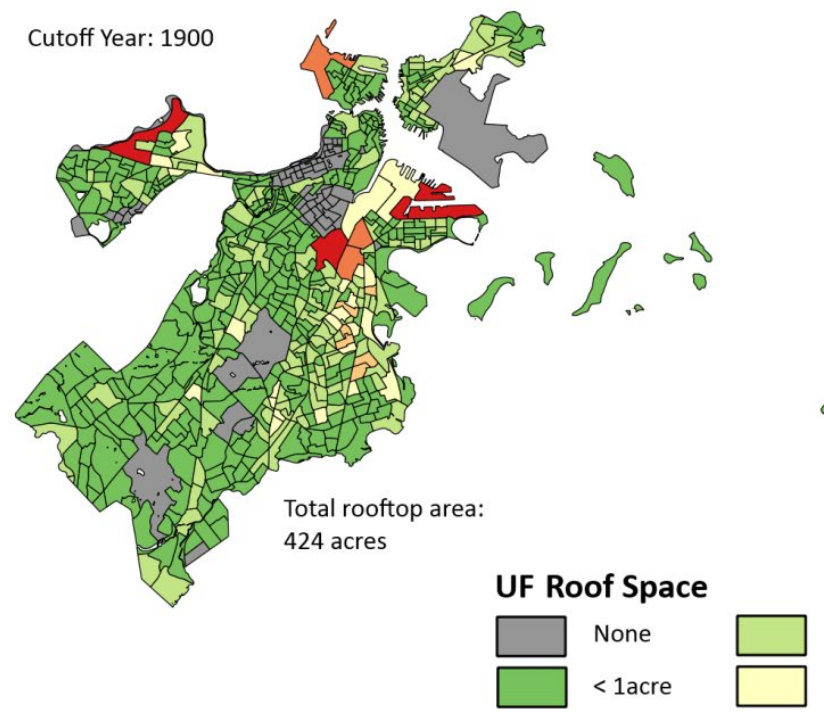

d)

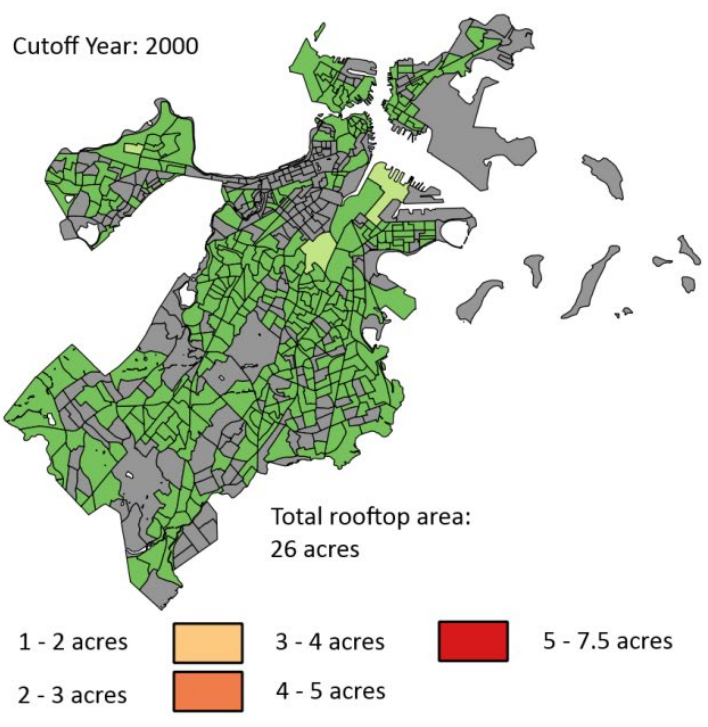

228 Figure 3a-d. Ground UF space in individual block-groups in Boston using (a) additive, (b)

229 subtractive and rooftop space using construction year cutoffs of (c) 1900 and (d) 2000.

\section{Environmental performance of $\mathbf{U F}$}

231 Figure 4a exhibits the changes in GWP potential through the introduction of UF into

232 Boston for the four scenarios. Results average all Monte-Carlo runs and all years for each 
233 scenario. The GWP(+) scenario provided $20 \%$ greater GHG reductions compared to the Land $(+)$

234 (18066 \pm 432 vs. $15045 \pm 523$ tons $\left.\mathrm{CO}_{2} \mathrm{e} / \mathrm{a}\right)$. With the subtractive method both GWP and land

235 optimizations approached each other ( 24000 tons $\left.\mathrm{CO}_{2} \mathrm{e} / \mathrm{a}\right)$ since they both produced until

236 Boston's demands for the UF crops are met, with slight differences due to allocation choices

237 (ground vs. roof) for select vegetables. In the best cases, UF reduced Boston's total food-borne

238 GWP burdens by approximately $1.1 \%$ (12\% of fruit and vegetable burdens) when limited by

239 space, and by $1.3 \%$ (15\% of fruit and vegetable burdens) when producing until vegetable

240 demands were met.
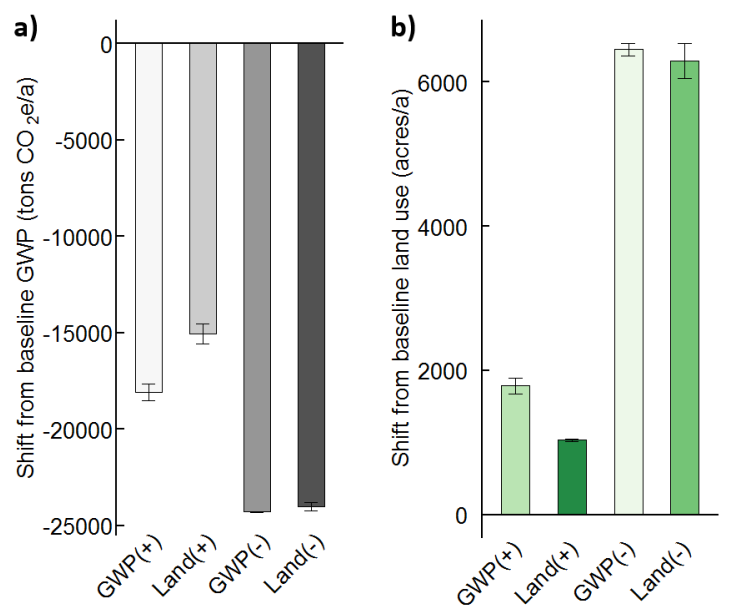

242 Figure 4. Impacts of UF on (a) GWP and (b) land use for all model scenarios. Error bars show

243 variance over all building construction cutoff years.

Figure $4 \mathrm{~b}$ shows the change in land use for the four scenarios. In all cases UF led to net

246 increases in land occupation. The Land $(+)$ optimization minimized these to $57 \%$ of those from

247 the GWP(+) scenario (1033 vs. 1786 acres/a increase). Akin to the GWP results, land use for

248 both optimization scenarios approached each other using the upper bound of UF space ( $\sim 6400$

249 acre/a increase). In the context of Boston's total food-related land occupation, these increases 
were a mere $0.07-0.5 \%$, and although hinting at UF's potential to worsen a city's environmental

251 performance, are not reason to outright discount UF as a food source for Boston. Land use

252 increases stemmed from the low-yield, ground-based UF which is the dominant farm type in the

253 model. Whilst UF frees some land beyond city boundaries, the practice requires more land within

254 city borders to produce an equivalent volume, highlighting the comparative advantage of

255 conventional production. Although already appropriated from the wild and hence imparting low

256 ecological 'costs' in converting to UF, it is worth considering whether vacant urban land is best

257 utilized for UF when solar farms net significantly greater environmental benefits per unit area,

258 particularly for GHG reductions ${ }^{21}$, but this could change in regions with lower GHG grid

259 intensities (the Northeast US is primarily fossil fuel supplied). Rooftop UF performed quite well

260 compared to conventional agriculture (exceptions being low yield vegetables where embodied

261 land use in capital is large), but the relatively small rooftop area cultivated was not enough to

262 counteract increases from ground UF. Although UF increased food related land use, the

263 conversion of urban space to farms could increase urban biodiversity ${ }^{49,50}$ and contribute to green

264 corridors through the city, potentially justifying the practice.

\section{UF impacts on Boston's energy and material metabolism}

266 Naturally, the more buildings employed for UF, the larger the building energy related

267 GWP reductions in Boston. In the Land(+) scenario, building energy savings accounted for 19\%

268 and 1\% of total GWP reductions to the city using 1900 and 2000 as construction cutoffs,

269 respectively, compared to $17 \%$ and $1 \%$ for the $\mathrm{GWP}(+)$ simulations. Both optimizations resulted

270 in approximately the same building energy GWP reductions $\left(3.2 \times 10^{6} \mathrm{~kg} \mathrm{CO}_{2} \mathrm{e}\right)$, but they took on

271 increased importance for the land optimization due to its poorer GWP performance. When the

272 simulations ran until Boston vegetable demands were met, building energy reductions 
273 contributed a maximum of 5\% to total GWP reductions as building UF took on a diminished

274 share of total production. In terms of contributions to total building energy demand, reduction

275 from UF's was in the single digits. UF's potential urban heat island mitigation was excluded

276 here, which could reduce ambient temperatures by $1-2^{\circ} \mathrm{C}^{51}$, affecting cooling energy loading.

277 However, cooling energy pales in comparison to heating demands in New England (1\% and 59\%

278 of total residential end use, respectively in Massachusetts ${ }^{43,44}$, hinting at the limited ability of

279 UF to affect baseline urban energy metabolism, although more detailed modeling is required.

280 Figures 5a-b outline UF's impacts on surface runoff and solid waste flows in Boston.

281 Building space was highly influential on these interactions since it is the majority of UF area that

282 shifts from non- to permeable and its significant compost needs due to soil losses and expanded

283 shale grow-media devoid of nutrients ${ }^{21}$. Here we focus on building cutoff years of 1900 and 2000

284 (other years shown in the supplementary information graphics). Figure 4a shows that the

$285 \mathrm{GWP}(+)$ and Land(+) simulations (averaged due to similarity) provided significantly greater

286 runoff retention, since they were forced to use all available building area. The subtractive

287 scenarios provided less runoff reduction as they favored ground UF when optimizing

288 (particularly the GWP(+) scenario) and did not convert any impermeable area on the ground to

289 UF. The maximum estimated runoff retention was $2.0 \mathrm{Mm}^{3} / \mathrm{a}$, or $2.0 \%$ of annual stormwater

$290 \quad\left(1.11 \mathrm{~m}\right.$ annual precipitation falling on $67.8 \mathrm{~km}^{2}$ impermeable area and $57.4 \mathrm{~km}^{2}$ permeable with

$29150 \%$ assumed retention $\left.{ }^{49}\right)$.

292 Yard and kitchen solid waste assimilation as compost (also averaged for land and GWP

293 optimizations) was highest for the subtractive scenarios $(\sim 12000$ tons/a), decreasing as building

294 space was removed to approximately 8200 tons/a by cutoff year 2000. Additive scenarios

295 provided lower waste assimilation capacity (10648-4026 tons/a) and were more sensitive to 
296 building space removal as this constituted a larger proportion of UF area. By our estimates UF

297 could absorb at most 9\% of Boston's municipal organic solid waste fraction at 2009 generation 298 rates $^{52}$.
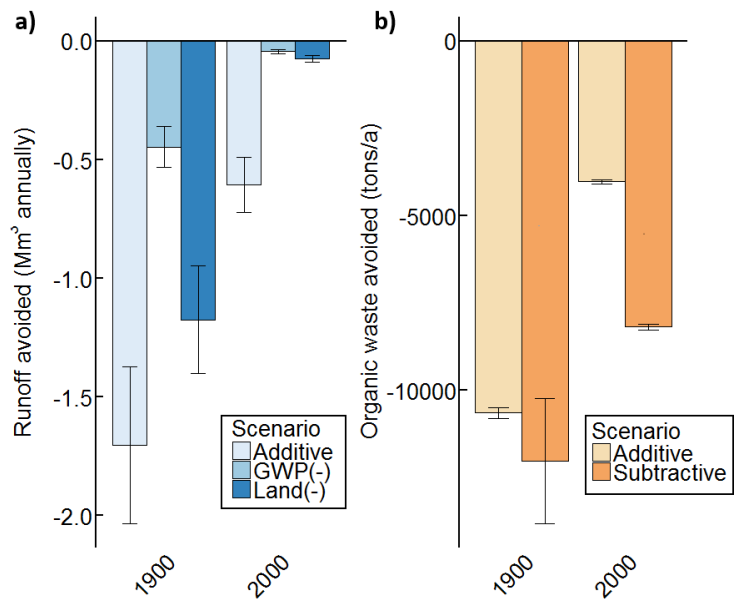

300 Figure 5a-b. Effects of UF on Boston's material metabolism for (a) runoff and (b) organic solid 301 waste uptake for cutoff years 1900 and 2000. Error bars display range for high and low retention 302 values for the runoff and variance over 100 Monte Carlo simulations for waste uptake.

303 Alternative motives for $\mathbf{U F}$

Given UF's meager improvements in food related GWP and potential exacerbation of

305 land use impacts, urban designers in the Northeast US should reconsider their enthusiasm for UF

306 as a component of an environmentally sustainable urban food system, especially compared to

307 higher environmental gains from other land applications ${ }^{21}$. Urban farms in the region do not

308 tackle the animal-sourced foods that drive dietary environmental burdens. Other cities in the

309 Global North are actively promoting reduced meat intake as explicit environmental initiatives,

310 recognizing the importance of diet, and not technology, as means to more sustainable cities ${ }^{53,54}$.

311 In a US context, shifting from average to vegetarian and vegan diets would reduce GWP by $30 \%$

312 and $50 \%$, respectively ${ }^{39}$ and reduce land use by a factor two or greater ${ }^{47}$. 
314 meagre, though the latter's ability to stymie sewage overflow events during heavy rains is

315 notable ${ }^{55}$. UF incorporated a sizeable amount of organic solid waste, although meaningful shifts

316 towards a circular metabolism should tackle wastewater management systems, where most

317 imported nutrients end up ${ }^{56,57}$.

318 Notwithstanding, UF is also often promoted as a social enterprise in the US Northeast ${ }^{7}$.

319 The slight environmental gains should be compared against performance in other domains to see

320 if current policies are justified given alternative motivations. In Boston, a significant percentage

321 of residents do not meet recommended fruit and vegetable guidelines, and some of the city's

322 neighborhoods have elevated poverty $\operatorname{rates}^{58}$. Here we test UF's potential contributions towards

323 alleviating these challenges.

324 Nutritional Improvements

325 UF's nutritional contributions were assessed as the percentage of USDA recommended

326 annual vegetable intake met for the three vegetable types grown by our case farms: 'dark green'

327 (e.g. spinach, kale, broccoli), 'red and orange' (tomatoes, carrots, squash) and 'other' (lettuce,

328 onions, cucumbers $)^{59}$. USDA guidelines for these vegetable types at different ages and sexes

329 were combined with census data to calculate Boston's total vegetable needs. We estimated that

330 Boston currently consumes $64 \%, 64 \%$ and $85 \%$ of its dark green, red/orange and other vegetable

331 needs, respectively (see Table S47 in the supplementary information). Nutrition optimization

332 algorithms were run for both additive $(+)$ and subtractive (-) grow area estimates, where the

333 farms supplied equal nutritional requirements for each vegetable type.

334 Figure 6 shows average nutritional output for the previous scenarios and nutrition

335 optimizations. Both GWP and land optimizations provided appreciable percentages of red/orange 
336 and other vegetable needs, but dark greens were not produced in volumes greater than $11 \%$ of

337 recommended intakes. The Nutrition(+) optimization reduced red/orange and other vegetable

338 production, but provided a fourfold jump in dark green cultivation, while the Nutrition(-)

339 scenario supplied all of Boston's needs in the three categories.

340

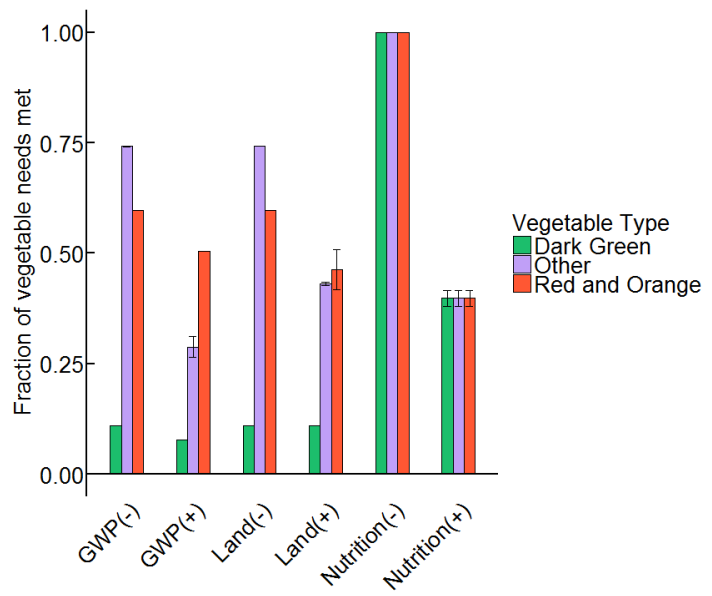

341 Figure 6. Fraction of vegetable needs met by UF in Boston for the different scenarios according

342 to USDA dietary guidelines.

343 In terms of GWP reduction the nutritional scenario was similar to the Land $(+)$ simulation

344 (15726 \pm 733 tons $\left.\mathrm{CO}_{2} \mathrm{e} / \mathrm{a}\right)$ and provided the largest reductions of all scenarios when producing

345 until all nutritional needs were assuaged $\left(39312 \pm 25\right.$ tons $\mathrm{CO}_{2} \mathrm{e} / \mathrm{a}$ or $2.9 \%$ of average dietary

346 GWP), since it substituted the greatest volume of conventional produce. When producing to meet

347 all nutritional demands, land use impacts were reduced relative to the other optimizations

348 (3746 \pm 77 acres/a), since the Nutrition(-) scenario grew significantly more dark green vegetables,

349 which provide high marginal land use reductions. The scale of interactions with the city

350 remained largely unchanged, but the Nutrition(-) scenario had $25 \%$ greater solid waste

351 assimilation capacity since cultivated area was the largest of all scenarios. 
We also tested when UF acts as an ancillary food supply that can be used to alleviate the

353 aforementioned gaps between USDA guidelines and current consumption. As UF would not

354 substitute conventional produce here, no crediting was provided to the city and full burdens of

355 UF production were ascribed to Boston. By our estimates Boston could actually close its

356 nutritional gap for these food groups within the UF space estimated by the additive method, with

357 the downside of increasing land use by $2608 \pm 89$ acres/a $(0.2 \%)$ and GWP by $2950 \pm 138$ tons

$358 \mathrm{CO}_{2} \mathrm{e} / \mathrm{a}(0.2 \%)$. However, the ecological costs should be weighed against the benefits of closing

359 nutritional gaps, particularly in inner city neighborhoods bereft of fresh vegetable choices where

360 lifestyle related diseases are more prominent ${ }^{60,61}$. Nutritional gaps would remain for other

361 vegetable types ('starchy' and 'legumes') and fruits, but promoting UF as a public health

362 measure appears justified.

\section{Economic benefits}

364 Lastly we looked at the ability for UF to provide economic returns to the block-groups

365 for all of the GWP, land and nutritional optimization scenarios. Because supplying Boston's

366 vegetable demands or nutritional needs required $\sim 50 \%$ and $64 \%$ of total UF area, respectively,

367 we also explored Boston's potential to export beyond its borders to the larger metropolitan area.

368 Vegetable prices were taken US Bureau of Labor Statistics and USDA data ${ }^{62,63}$. The 191 acres of

369 UF applied to surface parking in the additive scenarios were removed here, since this area

370 already generates revenue.

371 Figure 7a shows that when restricted to intra-block-group trading, estimated UF market

372 value was lowest $\left(\sim 1.5 \times 10^{7}\right.$ USD) for the GWP(-) and Land(-) trials, as more block-groups were

373 self-sufficient. Market value for internal trading is maximized $\left(\sim 4.9 \times 10^{7}\right.$ USD) when the model

374 aimed to meet its nutritional needs, as this left the most block-groups in production deficits, 
375 necessitating purchases from block-groups with surplus production capacity. Figure $7 \mathrm{~b}$ shows an

376 estimated market value of $\sim 1.6 \times 10^{8}$ USD when the city used all UF space, with exports to the

377 metropolitan region accounting for $\sim 90 \%$ of that when producing to meet current vegetable

378 demands and dropping to $67 \%$ when satisfying nutritional needs. Situating this within the

379 Boston-Cambridge-Newton metropolitan area, estimated UF market value amounted to less than

$380 \quad 0.5 \%$ of regional GDP ${ }^{64}$. Notwithstanding, Figure $7 \mathrm{c}$ maps potential UF revenue in the

381 Nutrition(-) scenario along with household poverty rates in Boston, demonstrating UF as a latent

382 revenue stream to some of Boston's impoverished neighborhoods. $\sim 2.5^{*} 10^{7}$ USD could be

383 generated in low income block-groups housing $\sim 81$ thousand residents (1/3 of Boston's residents

384 in low income blocks). However, most of the market value $\left(\sim 1.0^{*} 10^{8}\right.$ USD) would benefit blocks

385 with poverty rates below $25 \%$.

a)

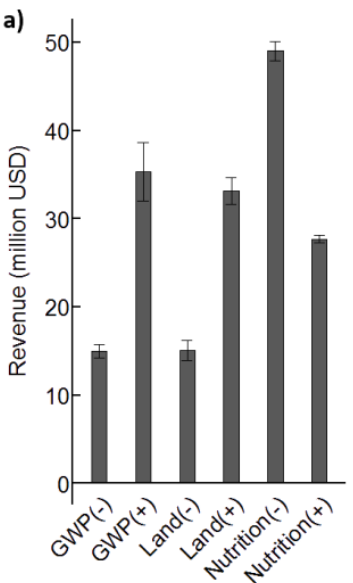

(No Exporting) b)

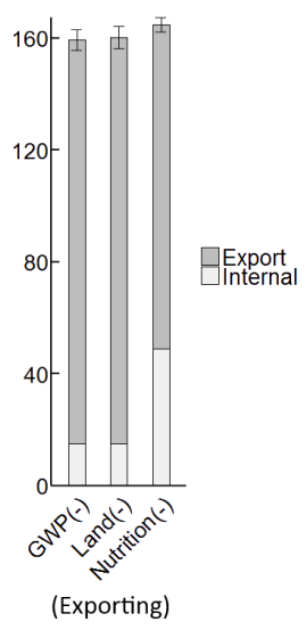

c)

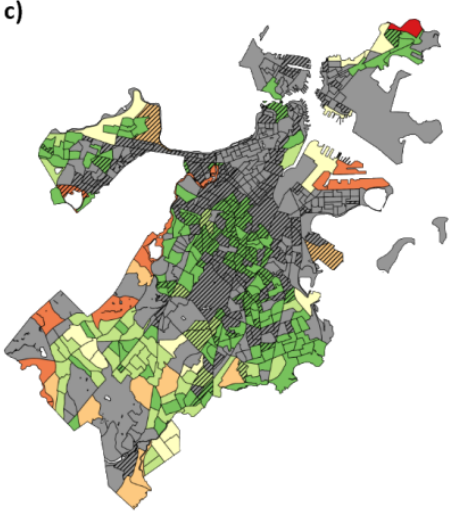

Nutrition(-) with exports
Poverty Rate

$\square<25 \%$

W/. $>25 \%$

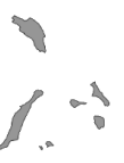

Market Value

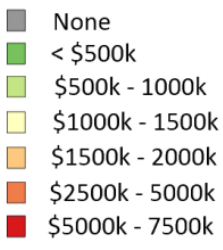

387 Figure 7a-c. Potential UF revenue in Boston when (a) limited to intra-block-group trading and

388 (b) exports outside of city allowed. (c) Revenue production (with exporting) in block-groups

389 after supplying cities nutritional needs with household poverty rates overlaid.

390 UF and study challenges 
392 well as aerial deposition of contaminants from the concentration of industry and traffic in urban

$393 \operatorname{areas}^{65,66}$. Of particular concern is the legacy of lead in soil from lead-containing fossil fuel

394 combustion, although minimal uptake outside of the root zone occurs, and oral intake can be

395 obviated through discarding of root portions and proper rinsing of edible portions ${ }^{67}$. Polycyclic

396 aromatic hydrocarbons pose a similar issue, more so from aerial deposition than plant uptake,

397 and can usually be made safe for consumption by rinsing edible portions ${ }^{67}$. Actual ingestion of

398 toxic substances through UF remains understudied, and is a serious concern despite these

399 positive signs. The presence of contamination is site specific, but it is correlated to age and

400 density of the city ${ }^{66}$, and in Northeast US cities the amount of current UF suitable area is

401 certainly lower than our estimates.

402 UF is also at odds with other more economically competitive land uses that are usually

403 preferred by municipal governments, further reducing long term production capacity ${ }^{68}$. Securing

404 UF's role as a nutrition source in the Northeast US will likely require more than making the

405 practice legal, but active protection of UF suitable space to avoid transitory UF application. This

406 could easily be done for city-owned vacant properties as a start.

407 By including potentially contaminated land in our models this study represents an

408 optimistic take on the potential for UF to affect a city's environmental performance. At the same

409 time, using process-based LCA for crop production may have underestimated the burdens of

410 both UF and substituted vegetables due to inventory gaps, depressing or inflating UF substitution

411 effects. Furthermore, UF practice is constantly evolving, with improvements to current systems

412 and new systems entering the market ${ }^{17}$. Although previous work has demonstrated that

413 technologically advanced urban farms in the study region are the most burdensome due to energy 
414 impacts $^{21}$, future developments might shift the balance in the opposite direction. Our findings are

415 only a snapshot of the current best-practices in the study region, which should be reevaluated as

416 UF technology and the region's electrical grid mix evolve. However, given the marginal impacts

417 of UF in this study, such shifts need to be seismic in order for UF contribution meaningfully to

418 making Northeast US urban food consumption more environmentally sustainable.

419 Results should also be viewed in light of Boston's relatively dense built form, which

420 produces high competition for the scarce open space remaining, reducing UF's tenability in the

421 city and its environmental and nutritional impacts. Less-dense or warmer Northeast US cities

422 may have greater production capacities per capita and resultant UF benefits, requiring care in

423 directly applying our results directly to other Northeast US cities. A more complete assessment

424 of local farming would look beyond political boundaries, including low-density suburbs and 425 peri-urban regions where higher production volumes are possible ${ }^{14,19}$. Regional food system 426 strategies, such as Vancouver, Canada's ${ }^{69}$, could help distinct political entities coordinate their 427 disparate land use regimes to maximize production and more effectively harness residual 428 resources, increasing local farming's benefits. Although focusing on Boston's geopolitical 429 boundaries precluded such a regional perspective, this study reveals the current limits of a lone, 430 urban municipality to reduce the environmental burdens of its food demands through technology.

431 Despite these methodological challenges, we have shown that when embedded within a 432 complex city system, UF's environmental performance is more nuanced than the previous 433 studies at the farm scale or using hypothetical UF data at the city scale would suggest. We have 434 demonstrated that it cannot be assumed that UF by default results in leaner supply chains. Policy 435 makers and other urban designers in the Northeast US will hopefully benefit from this and future 436 work when considering UF as a sustainable design intervention in the region. 
$438 \mathrm{CO}_{2} \mathrm{e}$, carbon dioxide equivalents; UF, urban farming; USDA, United States Department of

439 Agriculture; NHANES, National Health and Nutrition Examination Survey; MRIO, multi-

440 region-input-output; LCA, life cycle assessment; GWP, global warming potential.

\section{Acknowledgments}

442 The authors thank the reviewers and handling editor for helping strengthen the manuscript, Diana

443 Ivanova, Richard Wood, Sarah Schmidt and Jannick Schmidt for input on the MRIO modeling, 444 and Alexis Ernstoff for assistance with the NHANES data.

\section{Supporting Information Available}

446 Background data for the development of the food borne environmental footprints. Description of

447 methods for urban agriculture area estimates, urban agriculture life cycle assessment and

448 algorithms for urban farming proliferation in the city. Raw results from the models of impacts of 449 urban farming in the city.

\section{REFERENCES}

451 (1) Goldstein, B.; Birkved, M.; Fernández, J.; Hauschild, M. Surveying the Environmental 452 Footprint of Urban Food Consumption. J. Ind. Ecol. 2017, 21 (1), 151-165.

453 (2) Seto, K. C.; Ramankutty, N. Hidden linkages between urbanization and food systems. $454 \quad$ Science (80-. ). 2016, 352 (6288), 943-945.

455 (3) Tilman, D.; Clark, M. Global diets link environmental sustainability and human health. $456 \quad$ Nature 2014, 515, 518-522.

457 (4) Springmann, M.; Godfray, H. C. J.; Rayner, M.; Scarborough, P. Analysis and valuation of 
the health and climate change cobenefits of dietary change. Proc. Natl. Acad. Sci. U. S. A. 2016, 113 (15), 4146-4151.

(5) Koc, M.; Macrae, R.; Mougeot, L. J. A.; Welsh, J. For Hunger-proof Cities Sustainable Urban Food Systems; International Development Research Centre: Ottawa, CA, 1999.

(6) City of Milan. Milan Urban Food Policy Pact http://bit.ly/1SNW8PZ (accessed Feb 22, 2016).

Goldstein, M.; Bellis, J.; Morse, S.; Myers, A.; Ura, E. Urban Agriculture - a sixteen city survey of urban agriculture practices across the country; Atlanta, US, 2011.

(8) Armar-Klemesu, M. Urban agriculture and food security, nutrition and health. In Growing

(9) Schnitzler, W.; Holmer, R.; Heinrich, V. Urban agriculture-an essential element in feeding cities, growing food: urban agriculture on the policy agenda. A reader on urban agriculture; Bakker, N., Dubbling, M., Guendel, S., Sabel Koschella, U., de Zeeuw, H., Eds.; DSE, 2000; pp 99-117.

(10) Orsini, F.; Gasperi, D.; Marchetti, L.; Piovene, C.; Draghetti, S.; Ramazzotti, S.; Bazzocchi, G.; Gianquinto, G. Exploring the production capacity of rooftop gardens (RTGs) in urban agriculture: the potential impact on food and nutrition security, biodiversity and other ecosystem services in the city of Bologna. Food Secur. 2014, 6 (6), 781-792.

(11) Haberman, D.; Gillies, L.; Canter, A.; Rinner, V.; Pancrazi, L.; Martellozzo, F. The 477 
Information 2014, 3 (3), 1101-1117.

479 (12) Martellozzo, F.; Landry, J.-S.; Plouffe, D.; Seufert, V.; Rowhani, P.; Ramankutty, N. Urban agriculture: a global analysis of the space constraint to meet urban vegetable demand. Environ. Res. Lett. 2014, 9 (6), 64025.

(13) Kulak, M.; Graves, A.; Chatterton, J. Reducing greenhouse gas emissions with urban agriculture: A Life Cycle Assessment perspective. Landsc. Urban Plan. 2013, 111, 68-78.

(14) Benis, K.; Ferrão, P. Potential mitigation of the environmental impacts of food systems

(15) City of Boston. Article 89 Made Easy; 2014.

(16) Born, B.; Purcell, M. Avoiding the Local Trap: Scale and Food Systems in Planning Research. J. Plan. Educ. Res. 2006, 26 (2), 195-207.

(17) Goldstein, B.; Hauschild, M.; Fernández, J.; Birkved, M. Urban versus conventional agriculture, taxonomy of resource profiles: a review. Agron. Sustain. Dev. 2016, 36 (9), 119.

(18) Sanyé-Mengual, E.; Oliver-, J.; Montero, J. I.; Oliver-sola, J.; Montero, J. I.; Rieradevall, J. An environmenal and economic life cycle assessment of rooftop greenhouse (RTG) implementation in Barcelona, Spain. Assessing new forms of urban agriculture from the greenhouse structure to the final product level. Int. J. Life Cycle Assess. 2015, 20 (3), 350366. 
(19) Hall, G.; Rothwell, A.; Grant, T.; Isaacs, B.; Ford, L.; Dixon, J.; Kirk, M.; Friel, S. Potential environmental and population health impacts of local urban food systems under climate change: a life cycle analysis case study of lettuce and chicken. Agric. Food Secur. 2014, 3 $(1), 6$.

(20) Shiina, T.; Roy, H.; Nakamura, N.; Thammawong, M.; Orikasa, T. Life Cycle Inventory Analysis of Leafy Vegetables Grown in Two Types of Plant Factories. In Proceedings of the XXVIII International Horticultural Congress on Science and Horticulture for People; 2011; pp 115-122.

(21) Goldstein, B.; Hauschild, M.; Fernández, J.; Birkved, M. Testing the environmental performance of urban agriculture as a food supply in northern climates. J. Clean. Prod. 2016, 135, 984-994.

(22) Wood, R.; Stadler, K.; Bulavskaya, T.; Lutter, S.; Giljum, S.; de Koning, A.; Kuenen, J.; Schütz, H.; Acosta-Fernández, J.; Usubiaga, A.; et al. Global sustainability accountingdeveloping EXIOBASE for multi-regional footprint analysis. Sustain. 2015, 7 (1), 138-163.

(23) Turner, K.; Lenzen, M.; Wiedmann, T.; Barrett, J. Examining the global environmental impact of regional consumption activities - Part 1 : A technical note on combining input output and ecological footprint analysis. Ecol. Econ. 2007, 62, 37-44.

(24) Peters, G. P.; Hertwich, E. G. Production factors and pollution embodied in trade: Theoretical development; Trondheim, 2004.

(25) Hertwich, E. G.; Peters, G. P. Carbon footprint of nations: A global, trade-linked analysis. Environ. Sci. Technol. 2009, 43 (16), 6414-6420. 
519 (26) Hertwich, E. G. the Life Cycle Environmental Impacts of Consumption. Econ. Syst. Res.

$520 \quad$ 2011, $23(1), 27-47$.

521 (27) Ivanova, D.; Stadler, K.; Steen-Olsen, K.; Wood, R.; Vita, G.; Tukker, A.; Hertwich, E. G. 522 Environmental Impact Assessment of Household Consumption. J. Ind. Ecol. 2015, 0 (0), 1523

524 (28) Jones, C.; Kammen, D. M. Spatial distribution of U.S. household carbon footprints reveals 525

(29) Jones, C. M.; Kammen, D. M. Quantifying carbon footprint reduction opportunities for U.S. households and communities. Environ. Sci. Technol. 2011, 45 (9), 4088-4095.

(30) Clements, K.; Si, J. Engel's law, diet diversity and the quality of food consumption; 2015.

532 (32) U.S. Bureau of Labor Statistics. Consumer Expenditures in 2013; 2015.

533 (33) Levine, J. A. Poverty and obesity in the U.S. Diabetes 2011, 60 (11), 2667-2668.

534 (34) Centers for Disease Control and Prevention. About the National Health and Nutrition 535

(35) USDA. Food Availability (Per Capita) Data System http://www.ers.usda.gov/data537 products/food-availability-(per-capita)-data-system/.aspx (accessed Sep 23, 2016). 
(36) Garnett, T. Where are the best opportunities for reducing greenhouse gas emissions in the food system (including the food chain)? Food Policy 2011, 36, S23-S32.

(37) Muñoz, I.; Milà i Canals, L.; Fernández-Alba, A. R. Life cycle assessment of the average Spanish diet including human excretion. Int. J. Life Cycle Assess. 2010, 15 (8), 794-805.

(38) Weber, C. L.; Matthews, H. S. Food-miles and the relative climate impacts of food choices in the United States. Environ. Sci. Technol. 2008, 42 (10), 3508-3513.

(39) Heller, M. C.; Keoleian, G. a. Greenhouse Gas Emission Estimates of U.S. Dietary Choices and Food Loss. J. Ind. Ecol. 2015, 19 (3), 391-401.

(40) United States Department of Agriculture - National Agricultural Statistics Services. Vegetables 2014 Summary; 2015.

(41) Uhl, M.; Schiedt, L. Green Roof Storm Water Retention -Monitoring Results. In 11th International Conference on Urban Drainage; Edinburgh, 2008.

(42) Morgan, S.; Celik, S.; Retzlaff, W. Green Roof Storm-Water Runoff Quantity and Quality. J. Environ. Eng. 2013, 139 (2), 471-478.

(43) U.S. Energy Information Administration. Commercial buildings energy consumption survey http://www.eia.gov/consumption/commercial/ (accessed Aug 4, 2016).

(44) U.S. Energy Information Administration. Residential energy consumption survey https://www.eia.gov/consumption/residential/ (accessed Aug 4, 2016).

(45) La Roche, P.; Berardi, U. Comfort and energy savings with active green roofs. Energy Build. 2014, 82, 492-504. 
(46) Peters, C. J.; Wilkins, J. L.; Fick, G. W. Testing a complete-diet model for estimating the land resource requirements of food consumption and agricultural carrying capacity: The New York State example. Renew. Agric. Food Syst. 2006, 22 (2), 145-153.

(47) Peters, C. J.; Picardy, J.; Darrouzet-Nardi, A. F.; Wilkins, J. L.; Griffin, T. S.; Fick, G. W. Carrying capacity of U.S. agricultural land: Ten diet scenarios. Elementa 2016, 116 (4), 115.

(48) Eshel, G.; Shepon, a.; Makov, T.; Milo, R. Land, irrigation water, greenhouse gas, and reactive nitrogen burdens of meat, eggs, and dairy production in the United States. Proc. Natl. Acad. Sci. 2014, 1402183111-.

(49) Forman, R. Urban Ecology: Science of Cities; Cambridge University Press: Cambridge, UK, 2014.

(50) Oberndorfer, E.; Lundholm, J.; Bass, B.; Coffman, R. R.; Doshi, H.; Dunnett, N.; Gaffin, S.; Köhler, M.; Liu, K. K. Y.; Rowe, B. Green Roofs as Urban Ecosystems: Ecological Structures, Functions, and Services. Bioscience 2007, 57 (10), 823.

(51) Ackerman, K. The Potential for Urban Agriculture in New York City; New York, New York, USA, 2012.

(52) Massachusetts Department of Environmental Protection. Massachusetts 2010-2020 Solid Waste Master Plan; 2013.

(53) Martinko, K. New mayor of Turin, Italy, wants her city to go vegetarian http://www.treehugger.com/culture/new-mayor-turin-italy-wants-her-city-go- 
vegetarian.html (accessed Nov 20, 2016).

579

(54) Meat Free Mondays. City government of Oslo joins Meat Free Monday http://www.meatfreemondays.com/city-government-of-oslo-joins-meat-free-monday/ (accessed Nov 20, 2016).

(55) Boston Sewer and Water Commission. Combined sewer systems \& outfall maps http://www.bwsc.org/about_bwsc/systems/outfall_maps/outfall_maps.asp (accessed Aug 2, 2016).

(56) Forkes, J. Nitrogen balance for the urban food metabolism of Toronto, Canada. Resour. Conserv. Recycl. 2007, 52 (1), 74-94.

(57) Kalmykova, Y.; Harder, R.; Borgestedt, H.; Svanäng, I. Pathways and Management of Phosphorus in Urban Areas. J. Ind. Ecol. 2012, 16 (6), 928-939.

(58) Boston Public Health Commission. Health of Boston 2014-2015; Boston, Massachusetts, 2015.

(59) U.S. Department of Health and Human Services and U.S. Department of Agriculture. 20152020 Dietary Guidelines for Americans. 8th Edition; 2015.

(60) Gordon, C.; Purciel-Hill, M.; Ghai, N. R.; Kaufman, L.; Graham, R.; Van Wye, G. Measuring food deserts in New York City’s low-income neighborhoods. Heal. Place 2011, 17 (2), 696-700.

(61) Mejia, N.; Lightstone, A. S.; Basurto-Davila, R.; Morales, D. M.; Sturm, R. Neighborhood Food Environment, Diet, and Obesity Among Los Angeles County Adults, 2011. Prev. 
Chronic Dis. 2015, 12 (E143), 1-10.

602
(62) U.S. Bureau of Labor Statistics. Average Retail Food and Energy Prices, U.S. city average and Midwest Region http://www.bls.gov/regions/midatlantic/data/AverageRetailFoodAndEnergyPrices_USandMidwest_Table.htm (accessed Sep 30, 2016).

(63) United States Department of Agriculture - Agricultural Marketing Service. National Retail Report - Specialty Crops: Volume X - Number 40; 2016.

(64) U.S. Department of Commerce - Bureau of Economic Analysis. Regional Economic Accounts http://www.bea.gov/regional/index.htm (accessed Oct 31, 2016).

(65) Motelay-Massei, A.; Ollivon, D.; Garban, B.; Teil, M. J.; Blanchard, M.; Chevreuil, M. Distribution and spatial trends of PAHs and PCBs in soils in the Seine River basin, France. Chemosphere 2004, 55 (4), 555-565.

(66) Clark, H. F.; Brabander, D. J.; Erdil, R. M. Sources, sinks, and exposure pathways of lead in urban garden soil. J. Environ. Qual. 2006, 35 (6), 2066-2074.

(67) Wortman, S. E.; Lovell, S. T. Environmental challenges threatening the growth of urban agriculture in the United States. J. Environ. Qual. 2013, 42 (5), 1283-1294.

(68) Kaufman, J.; Bailkey, M. Farming Inside Cities : Entrepreneurial Urban Agriculture in the United States; 2000. 


\section{Supplementary Information}

Contributions of local farming to urban sustainability in the Northeastern United States

Benjamin P. Goldstein ${ }^{*}$, Michael Z. Hauschild ${ }^{\star}$, John E. Fernández ${ }^{\star}$, Morten Birkved ${ }^{\star}$

†Technical University of Denmark, Quantitative Sustainability Assessment Division, Produktionstorvet, Building 424, Kongens Lyngby, 2800, Denmark

¥Massachusetts Institute of Technology, Department of Architecture, 77 Massachusetts Avenue, 5-419, Cambridge, 02139, United States

*Corresponding author: Phone: +45 45254561; fax: +45 45933435; e-mail: bgol@dtu.dk 
34 Pages

65 Tables

13 Figures 


\section{Contents}

Linking MRIO food impacts to different nutritional categories

Determining food related GWP and land use impacts for Boston final consumption

Comparative performance of UF and conventional agriculture

UF Space Availability

Urban metabolism interactions

City-wide optimization simulations

Raw Results 


\section{Linking MRIO food impacts to different nutritional categories}

As outlined in the article, ascribing the embodied impacts of from food consumption to different food products is done using kilo calories. The starting point of the assessment are the individual categories of nutrition as outlined by the United States Department of Agriculture's (USDA) 2015-2020 dietary guidelines ${ }^{1}$.

USDA loss-adjusted food availability (LAFA) data ${ }^{2}$ provide kilo calories per nutritional equivalent for individual foods within the broader nutritional categories (e.g. kcal of broccoli per cup equivalent of 'dark green vegetables'), which are then used to develop availability weighted averages of kilo calories per nutritional equivalent. Food losses are also included in the weighted average, so that the kilo calories per nutritional unit approximate the amount of kilo calories provided by the economy for consumption and not just those actually consumed. Tables S1-19 outline the calculations of embodied kilo calories in the nutritional group.

\begin{tabular}{|c|c|c|c|c|c|c|c|}
\hline \multicolumn{8}{|c|}{ Table S1 - Vegetables: Dark Green } \\
\hline & \multirow[b]{2}{*}{$\begin{array}{l}\text { Loss from retail/ } \\
\text { institutional to } \\
\text { consumer level }\end{array}$} & \multicolumn{2}{|c|}{ Loss at consumer level } & \multirow[b]{2}{*}{$\begin{array}{c}\text { Kilo calories } \\
\text { available daily }\end{array}$} & \multirow[b]{2}{*}{$\begin{array}{l}\text { Food pattern } \\
\text { equivalents available } \\
\text { daily }\end{array}$} & \multirow[b]{2}{*}{$\begin{array}{l}\text { Produced kilo } \\
\text { calories }\end{array}$} & \multirow[b]{2}{*}{ Calorific density } \\
\hline & & Nonedible share & $\begin{array}{l}\text { Other (cooking } \\
\text { loss and uneaten } \\
\text { food) }\end{array}$ & & & & \\
\hline Component & -- Percent -- & -- Percent -- & -- Percent -- & -- Number - & -- Cups -- & kcal & $\begin{array}{l}\text { kcal produced/cup } \\
\text { consumed }\end{array}$ \\
\hline Fresh broccoli & 12 & 39 & 12 & 0.947 & 0.031 & 2.195 & 71.855 \\
\hline Fresh collard greens & 37 & 43 & 38 & 0.029 & 0.002 & 0.248 & 100.999 \\
\hline Fresh escarole & 48 & 14 & 24 & 0.030 & 0.004 & 0.092 & 24.664 \\
\hline Fresh kale & 39 & 39 & 38 & 0.022 & 0.001 & 0.154 & 200.145 \\
\hline Fresh leaf lettuce & 14 & 21 & 24 & 0.995 & 0.077 & 2.089 & 27.290 \\
\hline Fresh mustard greens & 64 & 7 & 38 & 0.023 & 0.002 & 0.116 & 75.014 \\
\hline Fresh spinach & 14 & 28 & 9 & 0.222 & 0.016 & 0.412 & 25.959 \\
\hline Fresh turnip greens & 41 & 30 & 38 & 0.031 & 0.002 & 0.164 & 95.294 \\
\hline Frozen broccoli & 6 & - & 12 & 0.585 & 0.011 & 0.707 & 62.863 \\
\hline Frozen spinach & 6 & - & 34 & 0.131 & 0.002 & 0.212 & 104.771 \\
\hline & & & & & & Weighted Average: & 43.617 \\
\hline
\end{tabular}

\begin{tabular}{|c|c|c|c|c|c|c|c|}
\hline & \multirow[b]{2}{*}{$\begin{array}{l}\text { Loss from retail/ } \\
\text { institutional to } \\
\text { consumer level }\end{array}$} & \multicolumn{2}{|c|}{ Loss at consumer level } & \multirow[b]{2}{*}{$\begin{array}{l}\text { Kilo calories } \\
\text { available daily }\end{array}$} & \multirow[b]{2}{*}{$\begin{array}{c}\text { Food pattern } \\
\text { equivalents available } \\
\text { daily }\end{array}$} & \multirow[b]{2}{*}{$\begin{array}{l}\text { Produced kilo } \\
\text { calories }\end{array}$} & \multirow[b]{2}{*}{ Calorific density } \\
\hline & & Nonedible share & $\begin{array}{l}\text { Other (cooking } \\
\text { loss and uneaten } \\
\text { food) }\end{array}$ & & & & \\
\hline Component & -- Percent -- & -- Percent -- & -- Percent -- & -- Number - & -- Cups -- & kcal & $\begin{array}{c}\text { kcal produced/cup } \\
\text { consumed }\end{array}$ \\
\hline Fresh artichokes & 19 & 60 & 18 & 0.149 & 0.002 & 0.840 & 428.102 \\
\hline Fresh asparagus & 9 & 47 & 18 & 0.084 & 0.003 & 0.264 & 85.157 \\
\hline Fresh green bell pepper & 8 & 18 & 39 & 0.427 & 0.014 & 1.078 & 75.645 \\
\hline Fresh Brussels sprouts & 19 & 10 & 12 & 0.094 & 0.002 & 0.149 & 59.965 \\
\hline Fresh cabbage & 14 & 20 & 24 & 1.093 & 0.050 & 2.271 & 45.721 \\
\hline Fresh cauliflower & 14 & 61 & 9 & 0.125 & 0.005 & 0.485 & 104.660 \\
\hline Fresh celery & 5 & 11 & 39 & 0.546 & 0.034 & 1.151 & 33.721 \\
\hline Fresh cucumbers & 6 & 27 & 32 & 0.332 & 0.024 & 0.864 & 36.376 \\
\hline Fresh eggplant & 21 & 19 & 26 & 0.101 & 0.005 & 0.233 & 46.222 \\
\hline Fresh garlic & 7 & 13 & 43 & 1.665 & 0.008 & 4.087 & 498.354 \\
\hline Fresh head lettuce & 9 & 16 & 24 & 1.557 & 0.097 & 2.843 & 29.216 \\
\hline Fresh mushrooms & 13 & 3 & 21 & 0.421 & 0.020 & 0.634 & 31.651 \\
\hline Fresh okra & 24 & 14 & 20 & 0.081 & 0.002 & 0.163 & 66.153 \\
\hline Canned olives & 6 & 0 & 25 & 2.126 & 0.014 & 3.016 & 219.858 \\
\hline Fresh onions & 10 & 10 & 43 & 4.283 & 0.067 & 10.099 & 150.892 \\
\hline Fresh radishes & 21 & 10 & 47 & 0.035 & 0.002 & 0.102 & 55.947 \\
\hline Fresh snap beans & 19 & 12 & 24 & 0.415 & 0.013 & 0.796 & 59.478 \\
\hline Fresh squash & 12 & 17 & 25 & 0.373 & 0.021 & 0.735 & 35.456 \\
\hline Canned asparagus & 6 & 0 & 2 & 0.026 & 0.001 & 0.028 & 49.935 \\
\hline Canned snap beans & 6 & 0 & 24 & 0.416 & 0.014 & 0.582 & 41.993 \\
\hline Canned cabbage & 6 & 0 & 16 & 0.083 & 0.003 & 0.105 & 34.195 \\
\hline Canned cucumbers & 6 & 0 & 3 & 0.876 & 0.011 & 0.960 & 89.384 \\
\hline Canned mushrooms & 6 & 0 & 9 & 0.250 & 0.006 & 0.292 & 45.593 \\
\hline Frozen asparagus & 6 & 0 & 26 & 0.007 & 0.000 & 0.011 & 46.003 \\
\hline Frozen snap beans & 6 & 0 & 24 & 0.444 & 0.012 & 0.622 & 53.191 \\
\hline Frozen cauliflower & 6 & 0 & 27 & 0.041 & 0.001 & 0.060 & 49.548 \\
\hline Dehydrated onions & 6 & 0 & 4 & 0.442 & 0.005 & 0.490 & 108.599 \\
\hline Fresh avocados & 9 & 26 & 32 & 2.516 & 0.011 & 6.607 & 622.305 \\
\hline & & & & & & Weighted Average & 88.607 \\
\hline
\end{tabular}

\begin{tabular}{|c|c|c|c|c|c|c|c|}
\hline \multicolumn{8}{|c|}{ Table S3 - Vegetables: Red and Orange } \\
\hline & \multirow{2}{*}{$\begin{array}{l}\text { Loss from retail/ } \\
\text { institutional to } \\
\text { consumer level }\end{array}$} & \multicolumn{2}{|c|}{ Loss at consumer level } & \multirow{2}{*}{$\begin{array}{c}\text { Kilo calories } \\
\text { available daily }\end{array}$} & \multirow{2}{*}{$\begin{array}{c}\text { Food pattern } \\
\text { equivalents } \\
\text { available daily }\end{array}$} & \multirow{2}{*}{$\begin{array}{l}\text { Produced kilo } \\
\text { calories }\end{array}$} & \multirow[b]{2}{*}{ Calorific density } \\
\hline & & Nonedible share & $\begin{array}{l}\text { Other (cooking loss } \\
\text { and uneaten food) }\end{array}$ & & & & \\
\hline Component & -- Percent -- & -- Percent -- & -- Percent -- & -- Number - & -- Cups -- & kcal & $\begin{array}{l}\text { kcal produced/cup } \\
\text { consumed }\end{array}$ \\
\hline Fresh red bell pepper & 8 & 18 & 39 & 0.427 & 0.014 & 1.077 & 75.644 \\
\hline Fresh carrots & 5 & 11 & 34 & 2.056 & 0.039 & 3.942 & 99.668 \\
\hline Fresh pumpkin & 11 & 30 & 69 & 0.012 & 0.001 & 1.436 & 3379.731 \\
\hline Fresh tomatoes & 13 & 9 & 7 & 3.370 & 0.082 & 4.621 & 56.220 \\
\hline Canned carrots & 6 & 0 & 31 & 0.136 & 0.003 & 0.210 & 55.504 \\
\hline Canned chili peppers & 6 & 0 & 4 & 1.022 & 0.035 & 1.132 & 32.136 \\
\hline Canned tomatoes & 6 & 0 & 28 & 3.750 & 0.098 & 5.541 & 56.146 \\
\hline Other canned vegetables & 6 & 0 & 16 & 0.503 & 0.010 & 0.637 & 62.056 \\
\hline Frozen carrots & 6 & 0 & 34 & 0.236 & 0.004 & 0.381 & 87.040 \\
\hline & & & & & & Weighted Average & 66.720 \\
\hline
\end{tabular}

\begin{tabular}{|c|c|c|c|c|c|c|c|}
\hline \multicolumn{8}{|c|}{ Table S4 - Vegetables: Starchy } \\
\hline & \multirow[b]{2}{*}{$\begin{array}{l}\text { Loss from retail/ } \\
\text { institutional to } \\
\text { consumer level }\end{array}$} & \multicolumn{2}{|c|}{ Loss at consumer level } & \multirow[b]{2}{*}{$\begin{array}{l}\text { Kilo calories } \\
\text { available daily }\end{array}$} & \multirow[b]{2}{*}{$\begin{array}{l}\text { Food pattern } \\
\text { equivalents available } \\
\text { daily }\end{array}$} & \multirow[b]{2}{*}{$\begin{array}{l}\text { Produced kilo } \\
\text { calories }\end{array}$} & \multirow[b]{2}{*}{ Calorific density } \\
\hline & & Nonedible share & $\begin{array}{l}\text { Other (cooking } \\
\text { loss and uneaten } \\
\text { food) }\end{array}$ & & & & \\
\hline Component & -- Percent -- & -- Percent -- & -- Percent -- & -- Number -- & -- Cups -- & $k c a l$ & $\begin{array}{l}\text { kcal produced/cup } \\
\text { consumed }\end{array}$ \\
\hline Fresh sweet corn & 1 & 64 & 32 & 0.361 & 0.003 & 9.091 & 3144.228 \\
\hline Fresh potatoes & 6 & 10 & 16 & 28.391 & 0.229 & 41.031 & 178.492 \\
\hline Fresh sweet potatoes & 14 & 28 & 44 & 1.172 & 0.010 & 4.879 & 474.417 \\
\hline
\end{tabular}




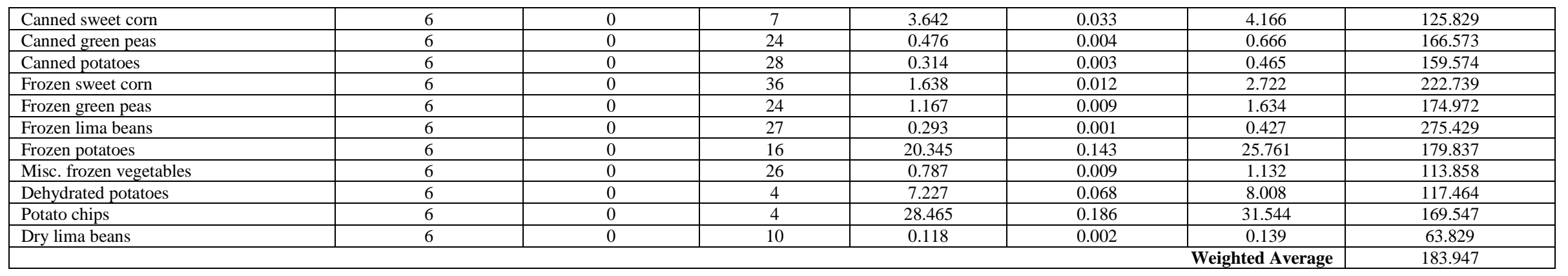

\begin{tabular}{|c|c|c|c|c|c|c|c|}
\hline abole s. Fruts - & \multirow[b]{2}{*}{$\begin{array}{l}\text { Loss from retail/ } \\
\text { institutional to } \\
\text { consumer level }\end{array}$} & \multicolumn{2}{|c|}{ Loss at consumer level } & \multirow[b]{2}{*}{$\begin{array}{l}\text { Kilo calories } \\
\text { available daily }\end{array}$} & \multirow[b]{2}{*}{$\begin{array}{l}\text { Food pattern } \\
\text { equivalents available } \\
\text { daily }\end{array}$} & \multirow[b]{2}{*}{$\begin{array}{l}\text { Produced kilo } \\
\text { calories }\end{array}$} & \multirow[b]{2}{*}{ Calorific density } \\
\hline & & Nonedible share & $\begin{array}{l}\text { Other (cooking } \\
\text { loss and uneaten } \\
\text { food) }\end{array}$ & & & & \\
\hline Component & -- Percent -- & -- Percent -- & -- Percent -- & -- Number -- & -- Cups -- & kcal & $\begin{array}{c}\text { kcal produced/cup } \\
\text { consumed }\end{array}$ \\
\hline Fresh oranges & 12 & 27 & 36 & 1.382 & 0.016 & 4.225 & 261.314 \\
\hline Fresh tangerines & 20 & 26 & 52 & 0.279 & 0.003 & 1.595 & 588.447 \\
\hline Fresh grapefruit & 13 & 50 & 20 & 0.286 & 0.003 & 1.095 & 313.588 \\
\hline Fresh lemons & 7 & 47 & 44 & 0.081 & 0.001 & 0.964 & 728.504 \\
\hline Fresh limes & 8 & 16 & 44 & 0.293 & 0.015 & 0.800 & 54.536 \\
\hline Fresh blueberries & 5 & 5 & 8 & 0.314 & 0.004 & 0.380 & 101.898 \\
\hline Fresh cranberries & 6 & 2 & 26 & 0.038 & 0.001 & 0.055 & 75.332 \\
\hline Fresh honeydew & 23 & 54 & 43 & 0.017 & 0.000 & 0.754 & 2697.253 \\
\hline Fresh kiwi & 13 & 14 & 45 & 0.108 & 0.001 & 0.302 & 212.224 \\
\hline Fresh raspberries & 10 & 4 & 20 & 0.074 & 0.001 & 0.108 & 93.312 \\
\hline Fresh strawberries & 10 & 6 & 35 & 1.220 & 0.025 & 2.291 & 92.591 \\
\hline Fresh watermelon & 17 & 48 & 13 & 1.573 & 0.034 & 4.845 & 141.727 \\
\hline Frozen blackberries & 6 & 0 & 40 & 0.042 & 0.000 & 0.074 & 171.986 \\
\hline Frozen raspberries & 6 & $\overline{0}$ & 24 & 0.191 & 0.003 & 0.268 & 102.184 \\
\hline Frozen strawberries & 6 & 0 & 24 & 0.151 & 0.003 & 0.211 & 72.788 \\
\hline Frozen other berries & 6 & 0 & 30 & 0.023 & 0.000 & 0.035 & 147.416 \\
\hline & & & & & & Veighted Average & 162.457 \\
\hline
\end{tabular}

\begin{tabular}{|l|}
\hline Table S6. Fruits - Juice \\
\hline
\end{tabular}

\begin{tabular}{|c|c|c|c|c|c|c|c|}
\hline & \multirow[b]{2}{*}{$\begin{array}{l}\text { Loss from retail/ } \\
\text { institutional to } \\
\text { consumer level }\end{array}$} & \multicolumn{2}{|c|}{ Loss at consumer level } & \multirow[b]{2}{*}{$\begin{array}{l}\text { Kilo calories } \\
\text { available daily }\end{array}$} & \multirow[b]{2}{*}{$\begin{array}{c}\text { Food pattern } \\
\text { equivalents available } \\
\text { daily }^{5}\end{array}$} & \multirow[b]{2}{*}{$\begin{array}{l}\text { Produced kilo } \\
\text { calories }\end{array}$} & \multirow[b]{2}{*}{ Calorific density } \\
\hline & & Nonedible share & $\begin{array}{l}\text { Other (cooking } \\
\text { loss and uneaten } \\
\text { food) }\end{array}$ & & & & \\
\hline Component & -- Percent -- & -- Percent -- & -- Percent -- & -- Number -- & -- Cups -- & kcal & $\begin{array}{l}\text { kcal produced/cup } \\
\text { consumed }\end{array}$ \\
\hline Orange juice & 6 & 0 & 10 & 18.071 & 0.161 & 21.361 & 132.388 \\
\hline Grapefruit juice & 6 & 0 & 10 & 1.252 & 0.013 & 1.480 & 113.475 \\
\hline Lemon juice & 6 & 0 & 10 & 0.283 & 0.005 & 0.335 & 63.830 \\
\hline Lime juice & 6 & 0 & 10 & 0.050 & 0.001 & 0.059 & 70.922 \\
\hline Apple juice & 6 & 0 & 10 & 9.712 & 0.085 & 11.480 & 134.752 \\
\hline Cranberry juice & 6 & 0 & 10 & 0.990 & 0.009 & 1.170 & 137.116 \\
\hline Grape juice & 6 & 0 & 10 & 3.163 & 0.021 & 3.739 & 179.669 \\
\hline Pineapple juice & 6 & 0 & 10 & 1.055 & 0.008 & 1.247 & 156.028 \\
\hline Prune juice & 6 & 0 & 32 & 0.223 & 0.001 & 0.349 & 284.731 \\
\hline & & & & & & Veighted Average & 135.490 \\
\hline
\end{tabular}

\begin{tabular}{|l}
\hline Table S7. Fruits: Other \\
\hline
\end{tabular}

\begin{tabular}{|c|c|c|c|c|c|c|c|}
\hline & \multirow[b]{2}{*}{$\begin{array}{l}\text { Loss from retail/ } \\
\text { institutional to } \\
\text { consumer level }\end{array}$} & \multicolumn{2}{|c|}{ Loss at consumer level } & \multirow[b]{2}{*}{$\begin{array}{l}\text { Kilo calories } \\
\text { available daily }{ }^{4}\end{array}$} & \multirow[b]{2}{*}{$\begin{array}{l}\text { Food pattern } \\
\text { equivalents } \\
\text { available daily }\end{array}$} & \multirow[b]{2}{*}{$\begin{array}{l}\text { Produced kilo } \\
\text { calories }\end{array}$} & \multirow[b]{2}{*}{ Calorific density } \\
\hline & & Nonedible share & $\begin{array}{l}\text { Other (cooking } \\
\text { loss and } \\
\text { uneaten food) }\end{array}$ & & & & \\
\hline Component & -- Percent -- & -- Percent -- & -- Percent -- & -- Number -- & -- Cups -- & kcal & $\begin{array}{l}\text { kcal produced/cup } \\
\text { consumed }\end{array}$ \\
\hline Fresh apples & 9 & 10 & 20 & 6.597 & 0.118 & 10.314 & 87.546 \\
\hline Fresh apricots & 35 & 7 & 10 & 0.045 & 0.001 & 0.084 & 141.924 \\
\hline Fresh bananas & 8 & 36 & 20 & 11.644 & 0.091 & 28.754 & 314.855 \\
\hline Fresh cantaloupe & 12 & 49 & 43 & 0.261 & 0.005 & 3.716 & 804.376 \\
\hline Fresh cherries & 4 & 9 & 51 & 0.294 & 0.003 & 0.766 & 221.060 \\
\hline Fresh grapes & 8 & 4 & 33 & 3.670 & 0.035 & 6.303 & 178.627 \\
\hline Fresh mangoes & 14 & 31 & 13 & 0.714 & 0.007 & 1.490 & 206.683 \\
\hline Fresh papaya & 55 & 33 & 20 & 0.116 & 0.002 & 0.547 & 292.434 \\
\hline Fresh peaches & 12 & 7 & 42 & 1.045 & 0.016 & 2.326 & 146.909 \\
\hline Fresh pears & 18 & 10 & 20 & 1.209 & 0.013 & 2.095 & 156.860 \\
\hline Fresh pineapple & 15 & 49 & 37 & 0.352 & 0.004 & 2.945 & 686.082 \\
\hline Fresh plums & 17 & 6 & 27 & 0.304 & 0.004 & 0.548 & 137.189 \\
\hline Canned apples & 6 & 0 & 8 & 2.210 & 0.022 & 2.555 & 117.946 \\
\hline Canned apricots & 6 & 0 & 27 & 0.032 & 0.001 & 0.046 & 72.865 \\
\hline Canned sweet cherries & 6 & 0 & 32 & 0.006 & 0.000 & 0.010 & 178.348 \\
\hline Canned tart cherries & 6 & 0 & 32 & 0.051 & 0.001 & 0.079 & 137.672 \\
\hline Canned peaches & 6 & 0 & 9 & 1.176 & 0.020 & 1.374 & 68.974 \\
\hline Canned pears & 6 & 0 & 9 & 1.099 & 0.015 & 1.285 & 83.002 \\
\hline Canned pineapple & 6 & 0 & 9 & 1.335 & 0.017 & 1.561 & 92.354 \\
\hline Canned plums & 6 & 0 & 26 & 0.007 & 0.000 & 0.011 & 146.636 \\
\hline Frozen blueberries & 6 & 0 & 29 & 0.158 & 0.002 & 0.237 & 118.370 \\
\hline Frozen sweet cherries & 6 & 0 & 29 & 0.149 & 0.001 & 0.223 & 346.119 \\
\hline Frozen tart cherries & 6 & 0 & 29 & 0.187 & 0.003 & 0.280 & 106.383 \\
\hline Frozen apples & 6 & 0 & 35 & 0.195 & 0.002 & 0.320 & 135.843 \\
\hline Frozen apricots & 6 & 0 & 35 & 0.015 & 0.000 & 0.025 & 135.843 \\
\hline Frozen peaches & 6 & 0 & 35 & 0.258 & 0.003 & 0.422 & 135.843 \\
\hline Frozen plums & 6 & 0 & 10 & 0.002 & 0.000 & 0.002 & 98.109 \\
\hline Dried apples & 6 & 0 & 11 & 0.287 & 0.003 & 0.343 & 124.313 \\
\hline Dried apricots & 6 & 0 & 11 & 0.312 & 0.002 & 0.373 & 187.664 \\
\hline Dried dates & 6 & 10 & 25 & 0.265 & 0.001 & 0.434 & 338.789 \\
\hline Dried figs & 6 & 0 & 25 & 0.203 & 0.001 & 0.288 & 263.830 \\
\hline Dried peaches & 6 & 0 & 11 & 0.118 & 0.001 & 0.141 & 228.305 \\
\hline Dried plums & 6 & 0 & 11 & 0.732 & 0.004 & 0.875 & 249.821 \\
\hline Raisins & 6 & 0 & 26 & 3.653 & 0.017 & 5.252 & 311.961 \\
\hline
\end{tabular}




\begin{tabular}{|c|c|c|c|c|c|c|c|}
\hline \multicolumn{8}{|l|}{ Table S8. Grains } \\
\hline & \multirow[b]{2}{*}{$\begin{array}{l}\text { Loss from retail/ } \\
\text { institutional to } \\
\text { consumer level }\end{array}$} & \multicolumn{2}{|c|}{ Loss at consumer level } & \multirow[b]{2}{*}{$\begin{array}{l}\text { Kilo calories } \\
\text { available daily }\end{array}$} & \multirow[b]{2}{*}{$\begin{array}{l}\text { Food pattern } \\
\text { equivalents } \\
\text { available daily }\end{array}$} & \multirow[b]{2}{*}{$\begin{array}{l}\text { Produced kilo } \\
\text { calories }\end{array}$} & \multirow[b]{2}{*}{ Calorific density } \\
\hline & & Nonedible share & $\begin{array}{l}\text { Other (cooking } \\
\text { loss and uneaten } \\
\text { food) }\end{array}$ & & & & \\
\hline Component & -- Percent -- & -- Percent -- & -- Percent -- & -- Number -- & $O z$ & kcal & $\begin{array}{c}\text { kcal produced/Oz } \\
\text { consumed }\end{array}$ \\
\hline White and whole wheat flour & 12 & 0 & 20 & 376.435 & 4.591 & 534.709 & 116.477 \\
\hline Rye flour & 12 & 0 & 20 & 2.152 & 0.027 & 3.056 & 113.636 \\
\hline Rice & 12 & 0 & 33 & 54.012 & 0.524 & 91.607 & 174.695 \\
\hline Corn flour and meal & 12 & 0 & 20 & 60.937 & 0.952 & 86.558 & 90.909 \\
\hline Corn hominy and grits & 12 & 0 & 20 & 28.810 & 0.389 & 40.923 & 105.114 \\
\hline Corn starch & 12 & 0 & 20 & 11.548 & 0.241 & 16.404 & 68.182 \\
\hline Barley products & 12 & 20 & 14 & 1.716 & 0.017 & 2.955 & 172.176 \\
\hline Oat products & 12 & 20 & 14 & 12.920 & 0.121 & 22.246 & 184.229 \\
\hline Durum flour & 12 & 0 & 20 & 40.140 & 0.379 & 57.018 & 150.568 \\
\hline & & & & & & eighted Average & 118.150 \\
\hline
\end{tabular}

\begin{tabular}{|c|c|c|c|c|c|c|c|}
\hline \multicolumn{8}{|l|}{ Table S9. Dairy - Cheese } \\
\hline & \multirow[b]{2}{*}{$\begin{array}{l}\text { Loss from retail/ } \\
\text { institutional to } \\
\text { consumer level }\end{array}$} & \multicolumn{2}{|c|}{ Loss at consumer level } & \multirow[b]{2}{*}{$\begin{array}{l}\text { Kilo calories } \\
\text { available daily }\end{array}$} & \multirow[b]{2}{*}{$\begin{array}{l}\text { Food pattern } \\
\text { equivalents available } \\
\text { daily }^{5}\end{array}$} & \multirow[b]{2}{*}{$\begin{array}{l}\text { Produced kilo } \\
\text { calories }\end{array}$} & \multirow[b]{2}{*}{ Calorific density } \\
\hline & & Nonedible share & $\begin{array}{l}\text { Other (cooking } \\
\text { loss and uneaten } \\
\text { food) }\end{array}$ & & & & \\
\hline Component & -- Percent -- & -- Percent -- & -- Percent -- & -- Number -- & Сup & $k c a l$ & $\begin{array}{c}\text { kcal produced/cup } \\
\text { consumed }\end{array}$ \\
\hline Cheddar cheese & 6 & 0 & 11 & 42.890 & 0.248 & 51.267 & 206.789 \\
\hline Other American cheese & 6 & 0 & 28 & 8.707 & 0.053 & 12.866 & 241.578 \\
\hline Provolone cheese & 6 & 0 & 14 & 3.751 & 0.025 & 4.640 & 184.315 \\
\hline Romano cheese & 6 & 0 & 8 & 1.154 & 0.007 & 1.335 & 190.796 \\
\hline Parmesan cheese & 6 & 0 & 8 & 2.818 & 0.016 & 3.258 & 206.984 \\
\hline Mozzarella cheese & 6 & 0 & 31 & 25.454 & 0.204 & 39.245 & 192.414 \\
\hline Ricotta cheese & 6 & 0 & 12 & 1.384 & 0.007 & 1.673 & 233.922 \\
\hline Other Italian cheese & 6 & 0 & 16 & 0.671 & 0.004 & 0.850 & 205.547 \\
\hline Swiss cheese & 6 & 0 & 50 & 2.803 & 0.017 & 5.965 & 344.681 \\
\hline Brick cheese & 6 & 0 & 40 & 0.063 & 0.000 & 0.111 & 280.142 \\
\hline Muenster cheese & 6 & 0 & 35 & 0.933 & 0.006 & 1.526 & 255.319 \\
\hline Blue cheese & 6 & 0 & 43 & 0.678 & 0.005 & 1.266 & 279.955 \\
\hline Other miscellaneous cheese & 6 & 0 & 42 & 3.554 & 0.023 & 6.519 & 287.417 \\
\hline Regular cottage cheese & 12 & 0 & 31 & 0.705 & 0.002 & 1.162 & 602.767 \\
\hline Low-fat cottage cheese & 12 & 0 & 4 & 1.063 & 0.003 & 1.259 & 384.706 \\
\hline & & & & & & Neighted Average & 214.276 \\
\hline
\end{tabular}

Table S10. Dairy - Milk

\begin{tabular}{|c|c|c|c|c|c|c|c|}
\hline & \multirow[b]{2}{*}{$\begin{array}{l}\text { Loss from retail/ } \\
\text { institutional to } \\
\text { consumer level }\end{array}$} & \multicolumn{2}{|c|}{ Loss at consumer level } & \multirow[b]{2}{*}{$\begin{array}{l}\text { Kilo calories } \\
\text { available daily }\end{array}$} & \multirow[b]{2}{*}{$\begin{array}{l}\text { Food pattern } \\
\text { equivalents } \\
\text { available daily }\end{array}$} & \multirow[b]{2}{*}{$\begin{array}{l}\text { Produced kilo } \\
\text { calories }\end{array}$} & \multirow[b]{2}{*}{ Calorific density } \\
\hline & & Nonedible share & $\begin{array}{l}\text { Other (cooking } \\
\text { loss and uneaten } \\
\text { food) }\end{array}$ & & & & \\
\hline Component & -- Percent -- & -- Percent -- & -- Percent -- & -- Number -- & Сир & kcal & $\begin{array}{l}\text { kcal produced/cup } \\
\text { consumed }\end{array}$ \\
\hline Plain whole milk & 12 & 0 & 20 & 28.079 & 0.188 & 39.885 & 211.648 \\
\hline 2 percent milk & 12 & 0 & 20 & 26.152 & 0.214 & 37.148 & 173.295 \\
\hline 1 percent milk & 12 & 0 & 20 & 8.044 & 0.079 & 11.426 & 144.886 \\
\hline Skim milk & 12 & 0 & 20 & 8.050 & 0.097 & 11.435 & 117.898 \\
\hline Whole flavored milk & 12 & 0 & 45 & 1.096 & 0.005 & 2.264 & 429.752 \\
\hline Low-fat flavored milk & 12 & 0 & 45 & 5.433 & 0.030 & 11.226 & 380.165 \\
\hline Buttermilk & 12 & 0 & 18 & 0.603 & 0.006 & 0.836 & 135.809 \\
\hline Ice cream & 12 & 0 & 24 & 24.519 & 0.060 & 36.661 & 613.038 \\
\hline Ice milk & 12 & 0 & 24 & 8.826 & 0.022 & 13.197 & 613.038 \\
\hline Other frozen & 12 & 0 & 33 & 4.895 & 0.021 & 8.303 & 388.399 \\
\hline Evap condensed canned whole milk & 12 & 0 & 15 & 15.950 & 0.094 & 21.323 & 225.936 \\
\hline Evap condensed bulk whole milk & 12 & 0 & 15 & 40.737 & 0.241 & 54.462 & 225.936 \\
\hline Evap condensed skim milk & 12 & 0 & 15 & 4.289 & 0.043 & 5.733 & 133.690 \\
\hline Dry whole milk & 1 & 0 & 41 & 0.562 & 0.004 & 0.962 & 272.214 \\
\hline Nonfat dry milk & 1 & 0 & 41 & 7.366 & 0.068 & 12.610 & 186.612 \\
\hline Dry buttermilk & 1 & 0 & 41 & 0.649 & 0.006 & 1.111 & 198.596 \\
\hline Eggnog & 12 & 0 & 51 & 0.044 & 0.000 & 0.101 & 347.866 \\
\hline & & & & & & Weighted Average & 228.156 \\
\hline
\end{tabular}

\begin{tabular}{|c|c|c|c|c|c|c|c|}
\hline \multicolumn{5}{|c|}{ ble S11. Dairy } & & & \\
\hline & $\begin{array}{l}\text { Loss from retail/ } \\
\text { institutional to } \\
\text { consumer level }\end{array}$ & Nonedible share & $\begin{array}{l}\text { Other (cooking } \\
\text { loss and uneaten } \\
\text { food) }\end{array}$ & $\begin{array}{l}\text { Kilo calories } \\
\text { available daily }\end{array}$ & $\begin{array}{l}\text { Food pattern } \\
\text { equivalents } \\
\text { available daily }\end{array}$ & $\begin{array}{l}\text { Produced kilo } \\
\text { calories }\end{array}$ & Calorific density \\
\hline Component & -- Percent -- & -- Percent -- & -- Percent -- & -- Number -- & Сир & kcal & $\begin{array}{l}\text { kcal produced/cup } \\
\text { consumed }\end{array}$ \\
\hline Yoghurt & 12 & 0 & 21 & 5.804 & 0.041 & 8.349 & 205.696 \\
\hline
\end{tabular}

\section{\begin{tabular}{|l}
\hline Table S12. Protein - Eggs \\
\hline
\end{tabular}}

\begin{tabular}{|c|c|c|c|c|c|c|c|}
\hline & \multirow[b]{2}{*}{$\begin{array}{l}\text { Loss from retail/ } \\
\text { institutional to } \\
\text { consumer level }\end{array}$} & \multicolumn{2}{|c|}{ Loss at consumer level } & \multirow[b]{2}{*}{$\begin{array}{l}\text { Kilo calories } \\
\text { available daily }{ }^{4}\end{array}$} & \multirow[b]{2}{*}{$\begin{array}{l}\text { Food pattern } \\
\text { equivalents } \\
\text { available daily }\end{array}$} & \multirow[b]{2}{*}{$\begin{array}{l}\text { Produced kilo } \\
\text { calories }\end{array}$} & \multirow[b]{2}{*}{ Calorific density } \\
\hline & & Nonedible share & $\begin{array}{l}\text { Other (cooking } \\
\text { loss and uneaten } \\
\text { food) }\end{array}$ & & & & \\
\hline Component & -- Percent -- & -- Percent -- & -- Percent -- & -- Number -- & $O z$ & kcal & $\begin{array}{c}\text { kcal produced/Oz } \\
\text { consumed }\end{array}$ \\
\hline Eggs & 9 & 12 & 13 & 37.843 & 0.485 & 63.978 & 131.868 \\
\hline
\end{tabular}




\begin{tabular}{|c|c|c|c|c|c|c|c|}
\hline & $\begin{array}{l}\text { institutional to } \\
\text { consumer level }\end{array}$ & Nonedible share & $\begin{array}{l}\text { Other (cooking } \\
\text { loss and uneaten } \\
\text { food) }\end{array}$ & available daily ${ }^{4}$ & $\begin{array}{c}\text { equivalents } \\
\text { available daily }\end{array}$ & calories & \\
\hline Component & -- Percent -- & -- Percent -- & -- Percent -- & -- Number -- & $O z$ & kcal & $\begin{array}{c}\text { kcal produced/Oz } \\
\text { consumed }\end{array}$ \\
\hline Fresh lima beans & 12 & 56 & 27 & 0.005 & 0.000 & 0.036 & 310.524 \\
\hline Dry Peas and lentils & 6 & 0 & 10 & 0.317 & 0.007 & 0.375 & 56.865 \\
\hline Dry black beans & 6 & 0 & 10 & 0.769 & 0.014 & 0.909 & 64.291 \\
\hline Dry great northern beans & 6 & 0 & 10 & 0.334 & 0.007 & 0.395 & 57.460 \\
\hline Dry navy beans & 6 & 0 & 10 & 1.450 & 0.025 & 1.714 & 68.246 \\
\hline Dry pinto beans & 6 & 0 & 10 & 4.078 & 0.069 & 4.821 & 69.708 \\
\hline Dry red kidney beans & 6 & 0 & 10 & 0.649 & 0.012 & 0.767 & 61.855 \\
\hline Other dry beans & 6 & 0 & 10 & 1.806 & 0.033 & 2.134 & 65.581 \\
\hline & & & & & & ghted Average & 66.797 \\
\hline
\end{tabular}

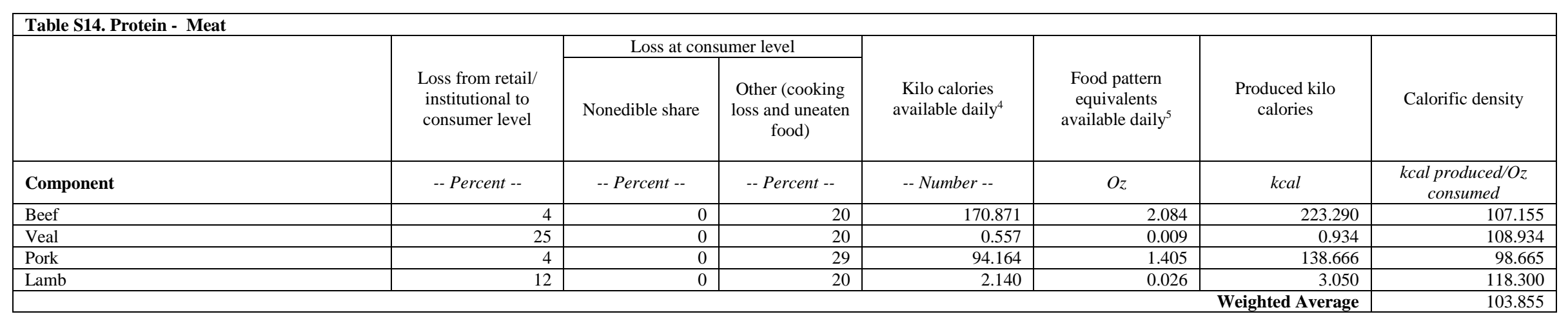

\begin{tabular}{|c|c|c|c|c|c|c|c|}
\hline & \multirow[b]{2}{*}{$\begin{array}{l}\text { Loss from retail/ } \\
\text { institutional to } \\
\text { consumer level }\end{array}$} & \multicolumn{2}{|c|}{ Loss at consumer level } & \multirow[b]{2}{*}{$\begin{array}{l}\text { Kilo calories } \\
\text { available daily }\end{array}$} & \multirow[b]{2}{*}{$\begin{array}{l}\text { Food pattern } \\
\text { equivalents } \\
\text { available daily }\end{array}$} & \multirow[b]{2}{*}{$\begin{array}{l}\text { Produced kilo } \\
\text { calories }\end{array}$} & \multirow[b]{2}{*}{ Calorific density } \\
\hline & & Nonedible share & $\begin{array}{l}\text { Other (cooking } \\
\text { loss and uneaten } \\
\text { food) }\end{array}$ & & & & \\
\hline Component & -- Percent -- & -- Percent -- & -- Percent -- & -- Number -- & $O z$ & kcal & $\begin{array}{l}\text { kcal produced/Oz } \\
\text { consumed }\end{array}$ \\
\hline Chicken & 4 & 0 & 15 & 141.470 & 2.143 & 173.318 & 80.858 \\
\hline Turkey & 3 & 0 & 35 & 20.498 & 0.380 & 32.666 & 86.055 \\
\hline
\end{tabular}

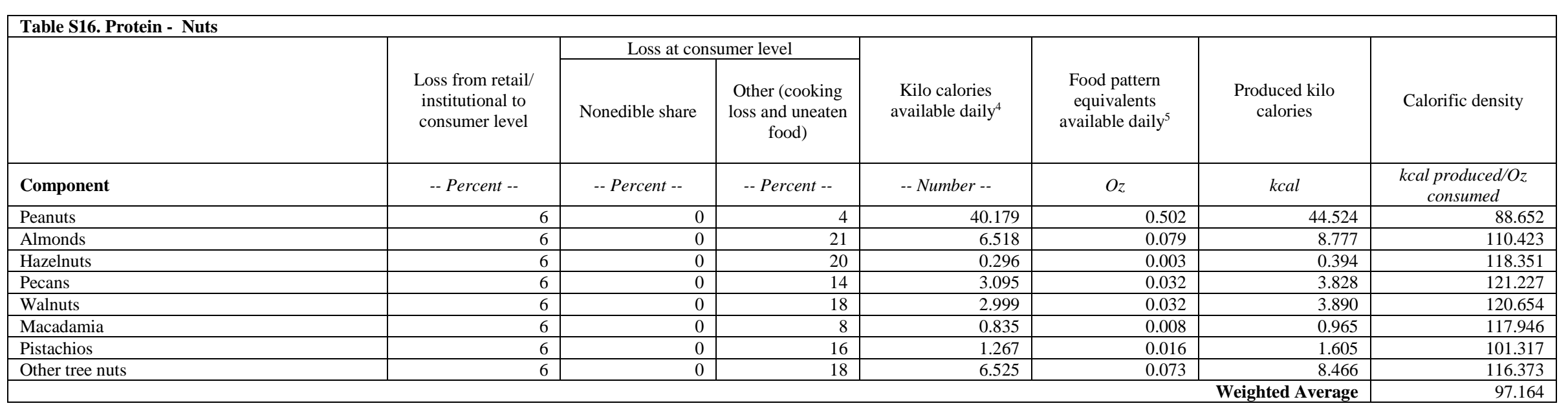

\begin{tabular}{|c|c|c|c|c|c|c|c|}
\hline & \multirow[b]{2}{*}{$\begin{array}{l}\text { Loss from retail/ } \\
\text { institutional to } \\
\text { consumer level }\end{array}$} & \multicolumn{2}{|c|}{ Loss at consumer level } & \multirow[b]{2}{*}{$\begin{array}{l}\text { Kilo calories } \\
\text { available daily }\end{array}$} & \multirow[b]{2}{*}{$\begin{array}{l}\text { Food pattern } \\
\text { equivalents } \\
\text { available daily }\end{array}$} & \multirow[b]{2}{*}{$\begin{array}{l}\text { Produced kilo } \\
\text { calories }\end{array}$} & \multirow[b]{2}{*}{ Calorific density } \\
\hline & & Nonedible share & $\begin{array}{l}\text { Other (cooking } \\
\text { loss and uneaten } \\
\text { food) }\end{array}$ & & & & \\
\hline Component & -- Percent -- & -- Percent -- & -- Percent -- & -- Number -- & $O z$ & kcal & $\begin{array}{l}\text { kcal produced/Oz } \\
\text { consumed }\end{array}$ \\
\hline Fresh and frozen fish & 9 & 0 & 40 & 5.598 & 0.158 & 10.218 & 64.802 \\
\hline Fresh and frozen shellfish & 9 & 0 & 40 & 3.265 & 0.131 & 5.998 & 45.925 \\
\hline Canned Salmon & 6 & 0 & 17 & 0.406 & 0.010 & 0.521 & 49.987 \\
\hline Canned Sardines & 6 & 0 & 36 & 0.304 & 0.005 & 0.505 & 98.072 \\
\hline Canned Tuna & 6 & 0 & 17 & 3.035 & 0.092 & 3.889 & 42.297 \\
\hline Canned shellfish & 6 & 0 & 17 & 0.419 & 0.015 & 0.536 & 35.888 \\
\hline Other canned fish & 6 & 0 & 17 & 0.402 & 0.010 & 0.515 & 49.987 \\
\hline Cured fish & 6 & 0 & 17 & 0.336 & 0.010 & 0.431 & 42.297 \\
\hline & & & & & & Weighted Average & 52.438 \\
\hline
\end{tabular}

\begin{tabular}{|c|c|c|c|c|c|c|c|}
\hline \multicolumn{8}{|l|}{ Table S18. Fats and Oils } \\
\hline & \multirow[b]{2}{*}{$\begin{array}{l}\text { Loss from retail/ } \\
\text { institutional to } \\
\text { consumer level }\end{array}$} & \multicolumn{2}{|c|}{ Loss at consumer level } & \multirow[b]{2}{*}{$\begin{array}{l}\text { Energy } \\
\text { content }\end{array}$} & \multirow[b]{2}{*}{$\begin{array}{l}\text { Per capita } \\
\text { availability adjusted } \\
\text { for loss }\end{array}$} & \multirow[b]{2}{*}{$\begin{array}{c}\text { Per capita } \\
\text { availability } \\
\text { adjusted for loss }\end{array}$} & \multirow[b]{2}{*}{ Calorific Density } \\
\hline & & Nonedible share & $\begin{array}{l}\text { Other (cooking } \\
\text { loss and uneaten } \\
\text { food) }\end{array}$ & & & & \\
\hline Component & -- Percent -- & -- Percent -- & -- Percent -- & $\mathrm{kcal} / \mathrm{g}$ & $\mathrm{g} / \mathrm{d}$ & $\mathrm{kcal} / \mathrm{d}$ & kcal produced/g consumed \\
\hline Butter & 7 & 0 & 35 & 9 & 2.815 & 25.333 & 14.888 \\
\hline Margarine & $\frac{1}{7}$ & 0 & $\frac{35}{35}$ & 9 & 2.037 & $\frac{18.3535}{18.335}$ & 14.0088 \\
\hline Lard & 50 & 0 & 35 & 9 & 0.651 & 5.858 & 27.692 \\
\hline Edible beef tallow & 50 & 0 & 35 & 9 & 1.189 & 10.705 & 27.692 \\
\hline Shortening & 21 & 0 & 35 & 9 & 13.368 & 120.316 & 17.527 \\
\hline Salad and cooking oils & 21 & 0 & 15 & 9 & 41.885 & 376.969 & 13.403 \\
\hline Other edible fats and oils & 5 & 0 & 25 & 9 & 1.488 & 13.388 & 12.632 \\
\hline Light cream & 12 & 0 & 12 & 9 & 1.556 & 14.000 & 11.622 \\
\hline Sour cream & 12 & 0 & 8 & 9 & 0.819 & 7.371 & 11.117 \\
\hline Cream cheese & 12 & 0 & 13 & 9 & 0.744 & 6.695 & 11.755 \\
\hline Eggnog & 12 & 0 & 51 & 9 & 0.011 & 0.098 & 20.872 \\
\hline
\end{tabular}




\begin{tabular}{|c|c|c|c|c|c|c|c|}
\hline \multicolumn{8}{|l|}{ Table S19. Sugars } \\
\hline & \multirow[b]{2}{*}{$\begin{array}{l}\text { Loss from retail/ } \\
\text { institutional to } \\
\text { consumer level }\end{array}$} & \multicolumn{2}{|c|}{ Loss at consumer level } & \multirow[b]{2}{*}{$\begin{array}{l}\text { Energy } \\
\text { content }\end{array}$} & \multirow[b]{2}{*}{$\begin{array}{c}\text { Per capita } \\
\text { availability } \\
\text { adjusted for loss }\end{array}$} & \multirow[b]{2}{*}{$\begin{array}{c}\text { Per capita } \\
\text { availability } \\
\text { adjusted for loss }\end{array}$} & \multirow[b]{2}{*}{ Calorific Density } \\
\hline & & $\begin{array}{l}\text { Nonedible } \\
\text { share }\end{array}$ & $\begin{array}{c}\text { Other } \\
\text { (cooking loss } \\
\text { and uneaten } \\
\text { food) }\end{array}$ & & & & \\
\hline Component & -- Percent -- & -- Percent -- & -- Percent -- & $\mathrm{kcal} / \mathrm{g}$ & $\mathrm{kcal} / \mathrm{d}$ & $\mathrm{kcal} / \mathrm{d}$ & kcal produced/kcal consumed \\
\hline Cane and beet sugar & 11 & 0 & 34 & - & 169.976 & 289.370 & 1.702 \\
\hline Edible syrups & 11 & 0 & 15 & - & 2.221 & 2.936 & 1.322 \\
\hline Honey & 11 & 0 & 15 & - & 3.340 & 4.415 & 1.322 \\
\hline High fructose corn sweetener & 11 & 0 & 34 & - & 155.278 & 264.349 & 1.702 \\
\hline Glucose & 11 & 0 & 34 & - & 38.070 & 64.811 & 1.702 \\
\hline Dextrose & 11 & 0 & 34 & - & 8.256 & 14.056 & 1.702 \\
\hline & & & & & & Veighted Average & 1.697 \\
\hline
\end{tabular}

We take the average US usual daily food intake for each nutritional group over the years 2007-2010 (in cup equivalents, ounce equivalents, etc) from the National Nutritional Health and Nutrition Examination Survey (NHANES) ${ }^{3}$ and multiply this by the population $(301,231,207)$ and kilo calories per serving nutritional component to arrive at the total calorific demand of the US population. Table S20 outlines these findings.

\begin{tabular}{|c|c|c|c|c|c|}
\hline Dietary Component & $\begin{array}{l}\text { NHANES Usual Daily } \\
\text { Intake }\end{array}$ & Unit & $\begin{array}{l}\text { kcal produced per } \\
\text { nutritional equivalent }\end{array}$ & Daily per capita kcal & $\begin{array}{l}\text { Annual per capita } \\
\text { kcal }\end{array}$ \\
\hline \multicolumn{6}{|l|}{ Vegetables } \\
\hline Dark Green & 0.1 & cup eq. & 43.61664 & 4.36166418 & 1592.007427 \\
\hline Red and Orange & 0.4 & cup eq. & 65.72036 & 26.2881426 & 9595.172043 \\
\hline Other & 0.5 & cup eq. & 88.60711 & 44.3035567 & 16170.79821 \\
\hline Starchy & 0.4 & cup eq. & 183.9475 & 73.5789929 & 26856.33242 \\
\hline \multicolumn{6}{|l|}{ Fruits } \\
\hline Citrus & 0.2 & cup eq. & 162.4571 & 32.4914191 & 11859.36796 \\
\hline Juice & 0.4 & cup eq. & 135.4902 & 54.1960631 & 19781.56303 \\
\hline Other & 0.5 & cup eq. & 183.6407 & 91.8203589 & 33514.431 \\
\hline \multicolumn{6}{|l|}{ Grains } \\
\hline Total & 6.3 & Oz eq. & 118.1499 & 744.344173 & 271685.6231 \\
\hline \multicolumn{6}{|l|}{ Dairy } \\
\hline Milk & 1 & cup eq. & 228.1564 & 228.156392 & 83277.083 \\
\hline Cheese & 0.7 & cup eq. & 214.2764 & 149.99351 & 54747.6311 \\
\hline Yoghurt & 0.1 & cup eq. & 205.6962 & 20.5696203 & 7507.911392 \\
\hline \multicolumn{6}{|l|}{ Protein } \\
\hline Meat & 2.5 & Oz eq. & 103.8546 & 259.636553 & 94767.34176 \\
\hline Poultry & 1.5 & Oz eq. & 81.64011 & 122.46017 & 44697.96204 \\
\hline Eggs & 0.5 & Oz eq. & 131.8681 & 65.9340659 & 24065.93407 \\
\hline Legumes & 0.5 & Oz eq. & 66.79709 & 33.3985472 & 12190.46974 \\
\hline Nuts & 0.6 & Oz eq. & 97.16419 & 58.2985134 & 21278.9574 \\
\hline Seafood & 0.5 & Oz eq. & 52.43769 & 26.2188457 & 9569.878694 \\
\hline \multicolumn{6}{|l|}{ Fats and Oils } \\
\hline Total & 56.8 & $\mathrm{~g}$ & 14.63028 & 830.99974 & 303314.905 \\
\hline \multicolumn{6}{|l|}{ Sugars } \\
\hline Total & 268 & kcal & 1.696806 & 454.744058 & 165981.5812 \\
\hline \multicolumn{6}{|l|}{ Beverages } \\
\hline Total ${ }^{*}$ & - & - & - & 447 & 163230 \\
\hline
\end{tabular}

* NHANES does not overtly track the kilo calories consumed through beverages. Estimated here as the difference between the US average total available kilo calories daily according to LAFA data for $2010^{2}$ (3769 kcal) and the sum of the food/juice intake estimated here.

Total GWP and land use impacts for US final demands were taken from the EXIOBASE v2.3 default final demand vector which represents consumption for the year 2007 (www.exiobase.eu). This only accounts for impacts for production, excluding final transport to the consumer. To account for transport impacts, the transport margins are taken from the EXIOBASE data for each product and multiplied by the final demands vector to generate the resulting final transport needs for each good in 2007 USD. The modal share is then taken from the United States Commodity Flow Survey for the year $2007^{4}$ using best judgement to link EXIOBASE products to the commodity groups covered in the survey. Table S21 displays the transport margins and modal share for each EXIOBASE product we include.

\begin{tabular}{|c|c|c|c|c|c|}
\hline 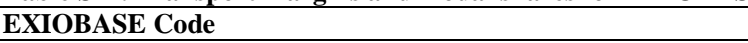 & Transport Margin & Modal Share - Road & Modal Share - Rail & Modal Share - Water & Modal Share - Air \\
\hline Paddy rice & 0.115998 & 0.488242 & 0.363942 & 0.147816 & 0 \\
\hline Wheat & 0.09643 & 0.488242 & 0.363942 & 0.147816 & 0 \\
\hline Cereal grains nec & 0.10418 & 0.488242 & 0.363942 & 0.147816 & 0 \\
\hline Vegetables, fruit, nuts & 0.131523 & 0.913812 & 0.043094 & 0.043094 & 0 \\
\hline Oil seeds & 0 & 0.913812 & 0.043094 & 0.043094 & 0 \\
\hline Sugar cane, sugar beet & 0 & 0.913812 & 0.043094 & 0.043094 & 0 \\
\hline Crops nec & 0 & 0.913812 & 0.043094 & 0.043094 & 0 \\
\hline Cattle & 0.006992 & 1 & 0 & 0 & 0 \\
\hline Pigs & 0 & 1 & 0 & 0 & 0 \\
\hline Poultry & 0.034421 & 1 & 0 & 0 & 0 \\
\hline Meat animals nec & 0 & 1 & 0 & 0 & 0 \\
\hline Animal products nec & 0.026805 & 0.991251 & 0.005661 & 0.003088 & 0.004117 \\
\hline Raw milk & 0 & 0.936752 & 0.063248 & 0 & 0 \\
\hline Fish and other fishing products; services incidental of fishing (05) & 0.061087 & 1 & 0 & 0 & 0 \\
\hline Products of meat cattle & 0.053237 & 1 & 0 & 0 & 0 \\
\hline Products of meat pigs & 0.063048 & 1 & 0 & 0 & 0 \\
\hline Products of meat poultry & 0.054744 & 1 & 0 & 0 & 0 \\
\hline Meat products nec & 0.077171 & 1 & 0 & 0 & 0 \\
\hline products of Vegetable oils and fats & 0.040329 & 0.972208 & 0.025695 & 0.002098 & 0.001049 \\
\hline Dairy products & 0.07116 & 0.936752 & 0.063248 & 0 & 0 \\
\hline Processed rice & 0.063115 & 0.969017 & 0.029915 & 0.001068 & 0 \\
\hline Sugar & 0.065962 & 0.972208 & 0.025695 & 0.002098 & 0.001049 \\
\hline Food products nec & 0.077061 & 0.972208 & 0.025695 & 0.002098 & 0.001049 \\
\hline Beverages & 0.101754 & 0.966173 & 0.033827 & 0 & 0 \\
\hline Fish products & 0.08422 & 1 & 0 & 0 & 0 \\
\hline
\end{tabular}

All transport is modeled using GWP and land use intensities for the US economy. The transport processes used here and their environmental intensities as taken from EXIOBASE are shown in Table S22.

Table S22. GWP and land use intensities for different transport modes 
Finally the MRIO calculations are performed; yielding the total production and transport related impacts related to US consumption for the year 2007. Table S23 outlines the results of the MRIO manipulations.

\begin{tabular}{|l|r|r|r|r|}
\hline \multicolumn{7}{|l|}{ Table S23. EXIOBASE results for 2007 US final consumption } \\
\hline
\end{tabular}

Allocating global warming potential (GWP) and land use impacts from the EXIOBASE is done through a concordance matrix matching nutritional groups to relevant product groups. Concordances are made based on the descriptions provided in the United Nations International Standard Industrial Classification of All Economic Activities classification codes ${ }^{5}$. The total impact from US final demand in 2007 in each relevant EXIOBASE product is divided amongst the kilo calories for all nutritional components ascribed to that product. Letting $I_{j}$ represent the total impacts (production and transport) from final demand for EXIOBASE food product $j$, and $C_{i}$ the total kilo calories produced of nutritional category $i$, then the impacts of the EXIOBASE product $j$ attributed to supplying a single kilo calorie of nutritional category $x, i_{x, j}$, is given by equation (1), where the denominator is the sum of kilo calories from all nutritional categories linked to that EXIOBASE product.

(1) $i_{x, j}=\frac{I_{j}}{\sum_{i=1}^{n} C_{i}}$

A single nutritional category could be matched to multiple EXIOBASE products, and hence, embodied impact per kilo calorie delivered in a nutritional category, $i_{x}$, is the sum of the components from each EXIOBASE product assigned to it, according to (2).

(2) $\quad i_{x}=\sum_{j=1}^{n} i_{x, j}$

Table S24 shows the concordance between different EXIOBASE products and the nutritional categories. Table S25 shows the embodied GWP and land use impacts per kilo calorie nutritional category produced.

\begin{tabular}{|l|l|}
\hline \multicolumn{2}{|l|}{ Table S24. Concordance matrix between EXIOBASE products and nutritional categories } \\
\hline EXIOBASE Code & USDA Nutritional Category \\
\hline Poultry & Poultry, Eggs \\
\hline Products of meat poultry & Poultry \\
\hline Cattlle & Meat \\
\hline Products of meat cattle & Meat \\
\hline Products of meat pigs & Meat \\
\hline Pigs & Meat \\
\hline Fish and other fishing products; services incidental of fishing & Fish and Seafood \\
\hline Fish products & Fish and Seafood \\
\hline Meat products nec & Meat \\
\hline Animal products nec & Poultry, Meat, Fish and Seafood, Milk, Cheese, Yoghurt, Eggs \\
\hline Dairy products & Milk, Cheese, Yoghurt \\
\hline Processed rice & Grains \\
\hline Paddy rice & Grains \\
\hline Cereal grains nec & Grains \\
\hline Wheat & Grains \\
\hline Products of Vegetable oils and fats & Fars and Oils \\
\hline Vegetables, fruit, nuts & Dark Green Vegetables, Red and Orange Vegetables, Starchy Vegetables, Citrus Fruits, Juice, Other Fruits, Nuts \\
\hline Sugar & Sugars \\
\hline Beverages & Beverages, Milk, Juice \\
\hline Crops nec & Other Vegetables, Starchy Vegetables \\
\hline Food products nec & Dark Green Vegetables, Red and Orange Vegetables, Starchy Vegetables, Citrus Fruits, Juice, Other Fruits, Nuts, \\
\hline
\end{tabular}

\begin{tabular}{|c|c|c|c|c|}
\hline USDA Nutritional Category & $\begin{array}{l}\text { GWP }\left(\mathrm{kg} \mathrm{CO}_{2} \mathrm{e} / \mathrm{kcal} \text { produced }\right) \text { - } \\
\text { production }\end{array}$ & $\begin{array}{l}\mathrm{GWP}\left(\mathrm{kg} \mathrm{CO}_{2} \mathrm{e} / \mathrm{kcal} \text { produced }\right) \text { - } \\
\text { transport }\end{array}$ & $\begin{array}{l}\text { Land Use (km²/ kcal produced) - } \\
\text { production }\end{array}$ & $\begin{array}{l}\text { Land Use (km²/ kcal produced) - } \\
\text { transport }\end{array}$ \\
\hline Poultry & 0.003409 & $9.90 \mathrm{E}-05$ & $1.09 \mathrm{E}-08$ & $3.39 \mathrm{E}-11$ \\
\hline Citrus, melons, berries & 0.001418 & 0.00012 & $1.12 \mathrm{E}-08$ & $4.18 \mathrm{E}-11$ \\
\hline Other Fruits & 0.001418 & 0.00012 & $1.12 \mathrm{E}-08$ & $4.18 \mathrm{E}-11$ \\
\hline Meat & 0.006777 & $9.32 \mathrm{E}-05$ & $2.23 \mathrm{E}-08$ & $3.20 \mathrm{E}-11$ \\
\hline Grains & 0.000731 & $2.57 \mathrm{E}-05$ & $3.27 \mathrm{E}-09$ & $9.43 \mathrm{E}-12$ \\
\hline Dark Greens & 0.001418 & 0.00012 & $1.12 \mathrm{E}-08$ & $4.18 \mathrm{E}-11$ \\
\hline Yoghurt & 0.001854 & $6.37 \mathrm{E}-05$ & $8.31 \mathrm{E}-09$ & $2.44 \mathrm{E}-11$ \\
\hline Red and Orange & 0.001418 & 0.00012 & $1.12 \mathrm{E}-08$ & $4.18 \mathrm{E}-11$ \\
\hline Sugars & 0.00071 & $2.55 \mathrm{E}-05$ & $3.08 \mathrm{E}-09$ & $9.19 \mathrm{E}-12$ \\
\hline Nuts & 0.001418 & 0.00012 & $1.12 \mathrm{E}-08$ & $4.18 \mathrm{E}-11$ \\
\hline Cheese & 0.001854 & $6.37 \mathrm{E}-05$ & $8.31 \mathrm{E}-09$ & $2.44 \mathrm{E}-11$ \\
\hline Fish and Seafood & 0.002473 & 0.000119 & $6.64 \mathrm{E}-09$ & $4.07 \mathrm{E}-11$ \\
\hline
\end{tabular}




\begin{tabular}{|c|c|c|c|c|}
\hline Juice & 0.002417 & 0.000188 & $1.40 \mathrm{E}-08$ & $6.70 \mathrm{E}-11$ \\
\hline Beverages & 0.001647 & 9.14E-05 & $5.72 \mathrm{E}-09$ & $3.36 \mathrm{E}-11$ \\
\hline Starchy & 0.001491 & 0.00012 & $1.57 \mathrm{E}-08$ & $4.18 \mathrm{E}-11$ \\
\hline Legumes and Soy & 0.000648 & $2.36 \mathrm{E}-05$ & $2.86 \mathrm{E}-09$ & $8.48 \mathrm{E}-12$ \\
\hline Other Vegetables & 0.001491 & 0.00012 & $1.57 \mathrm{E}-08$ & $4.18 \mathrm{E}-11$ \\
\hline Fats and Oils & 0.000745 & $2.48 \mathrm{E}-05$ & $3.56 \mathrm{E}-09$ & $8.92 \mathrm{E}-12$ \\
\hline Milk & 0.002853 & 0.000132 & $1.12 \mathrm{E}-08$ & 4.95E-11 \\
\hline Eggs & 0.000987 & $2.85 \mathrm{E}-05$ & $4.27 \mathrm{E}-09$ & $1.01 \mathrm{E}-11$ \\
\hline
\end{tabular}

\section{Determining food related GWP and land use impacts for Boston final consumption}

Embodied kilo calories per nutritional serving (Tables S1-19) can be connected with the GWP and land use impacts per kilo calorie nutritional category delivered to market (Table S25) to estimate environmental pressure exerted by different levels of food consumption. We use the NHANES 2007-2010 usual daily intake data for different demographics and US census data to estimate Boston's food related environmental burdens for the year 2010.

Table S26 shows the usual daily intake for different population segments based on sex and age, which when multiplied by 365 provide estimates of annual food demands for US citizens. It should be noted that NHANES, being self-reported, is plagued by underreporting by participants, particularly in foods that have negative health stigmas attached to them (red meat, sugar, highly processed foods, etc.) and is considered at the lower end of food consumption estimates ${ }^{6}$. Notwithstanding the above shortcoming, NHANES provides the most comprehensive and consistent data for US food consumption, with the added benefit of recording including important demographics data, and is therefore chosen here to model Boston's consumption.

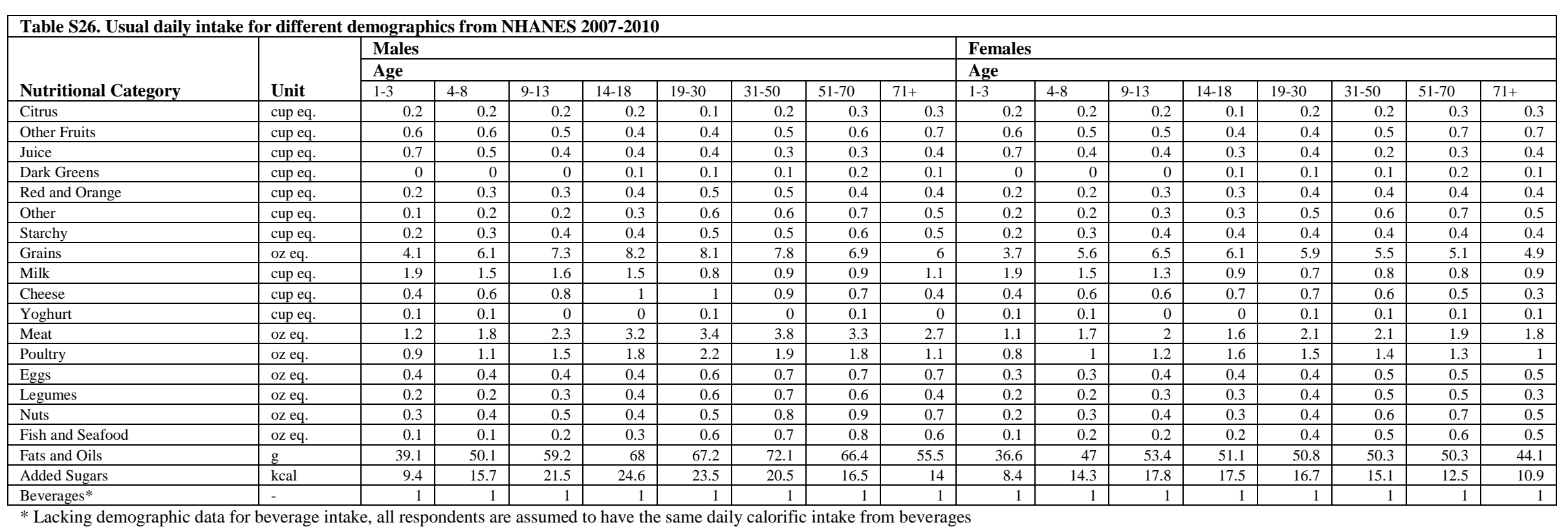

To move from NHANES usual daily intake to annual environmental impacts for a nutritional category, $Y_{x}$, the usual daily intake for nutritional component $x, U D I_{x}$, is combined with the produced kilo calories per nutritional unit, $k c a l_{x}$, and the impacts per kilo calorie supplied to the market, $i_{x}$, and corrected for the number of days in a year:

(3) $\quad Y_{x}=\left(U D I_{x} \times k c a l_{x} \times i_{x}\right) \times 365$

Tables S27 and S28 show food related GWP and land use impacts for different demographics, respectively.

\begin{tabular}{|c|c|c|c|c|c|c|c|c|c|c|c|c|c|c|c|c|}
\hline \multirow[b]{3}{*}{ Nutritional Category } & \multicolumn{8}{|l|}{ Males } & \multicolumn{8}{|l|}{ Females } \\
\hline & \multicolumn{8}{|l|}{ Age } & \multicolumn{8}{|l|}{ Age } \\
\hline & $1-3$ & $4-8$ & $9-13$ & $14-18$ & $19-30$ & $31-50$ & $51-70$ & $71+$ & $1-3$ & $4-8$ & $9-13$ & $14-18$ & 19-30 & $31-50$ & $51-70$ & $71+$ \\
\hline $\begin{array}{l}\text { Poultry } \\
\end{array}$ & 91.4 & 111.7 & 152.4 & 182.9 & 223.5 & 193.0 & 182.9 & 111.7 & 81.3 & 101.6 & 121.9 & 162.5 & 152.4 & 142.2 & 132.1 & 101.6 \\
\hline Citrus & 16.8 & 16.8 & 16.8 & 16.8 & 8.4 & 16.8 & 25.2 & 25.2 & 16.8 & 16.8 & 16.8 & 8.4 & 16.8 & 16.8 & 25.2 & 25.2 \\
\hline Fish and Seafood & 4.7 & 4.7 & 9.5 & 14.2 & 28.4 & 33.1 & 37.9 & 28.4 & 4.7 & 9.5 & 9.5 & 9.5 & 18.9 & 23.7 & 28.4 & 23.7 \\
\hline Other Fruits & 57.0 & 57.0 & 47.5 & 38.0 & 38.0 & 47.5 & 57.0 & 66.5 & 57.0 & 47.5 & 47.5 & 38.0 & 38.0 & 47.5 & 66.5 & 66.5 \\
\hline Meat & 308.3 & 462.4 & 590.9 & 822.1 & 873.5 & 976.2 & 847.8 & 693.6 & 282.6 & 436.7 & 513.8 & 411.0 & 539.5 & 539.5 & 488.1 & 462.4 \\
\hline Grains & 129.2 & 192.2 & 230.0 & 258.4 & 255.2 & 245.8 & 217.4 & 189.1 & 116.6 & 176.5 & 204.8 & 192.2 & 185.9 & 173.3 & 160.7 & 154.4 \\
\hline Dark Greens & 0.0 & 0.0 & 0.0 & 2.3 & 2.3 & 2.3 & 4.5 & 2.3 & 0.0 & 0.0 & 0.0 & 2.3 & 2.3 & 2.3 & 4.5 & 2.3 \\
\hline Red and Orange & 6.8 & 10.2 & 10.2 & 13.6 & 17.0 & 17.0 & 13.6 & 13.6 & 6.8 & 6.8 & 10.2 & 10.2 & 13.6 & 13.6 & 13.6 & 13.6 \\
\hline Sugars & 4.1 & 6.9 & 9.5 & 10.8 & 10.3 & 9.0 & 7.3 & 6.2 & 3.7 & 6.3 & 7.8 & 7.7 & 7.3 & 6.6 & 5.5 & 4.8 \\
\hline Nuts & 15.1 & 20.1 & 25.1 & 20.1 & 25.1 & 40.2 & 45.3 & 35.2 & 10.1 & 15.1 & 20.1 & 15.1 & 20.1 & 30.2 & 35.2 & 25.1 \\
\hline Milk & 451.4 & 356.4 & 380.1 & 356.4 & 190.1 & 213.8 & 213.8 & 261.3 & 451.4 & 356.4 & 308.8 & 213.8 & 166.3 & 190.1 & 190.1 & 213.8 \\
\hline Cheese & 58.0 & 87.0 & 116.0 & 145.0 & 145.0 & 130.5 & 101.5 & 58.0 & 58.0 & 87.0 & 87.0 & 101.5 & 101.5 & 87.0 & 72.5 & 43.5 \\
\hline Juice & 83.7 & 59.8 & 47.8 & 47.8 & 47.8 & 35.9 & 35.9 & 47.8 & 83.7 & 47.8 & 47.8 & 35.9 & 47.8 & 23.9 & 35.9 & 47.8 \\
\hline Beverages & 268.9 & 268.9 & 268.9 & 268.9 & 268.9 & 268.9 & 268.9 & 268.9 & 268.9 & 268.9 & 268.9 & 268.9 & 268.9 & 268.9 & 268.9 & 268.9 \\
\hline Fats and Oils & 155.5 & 199.2 & 235.4 & 270.4 & 267.2 & 286.7 & 264.0 & 220.7 & 145.5 & 186.9 & 212.3 & 203.2 & 202.0 & 200.0 & 200.0 & 175.4 \\
\hline Legumes and Soy & 3.2 & 3.2 & 4.7 & 6.3 & 9.5 & 11.1 & 9.5 & 6.3 & 3.2 & 3.2 & 4.7 & 4.7 & 6.3 & 7.9 & 7.9 & 4.7 \\
\hline Other Vegetables & 4.8 & 9.6 & 9.6 & 14.5 & 28.9 & 28.9 & 33.8 & 24.1 & 9.6 & 9.6 & 14.5 & 14.5 & 24.1 & 28.9 & 33.8 & 24.1 \\
\hline Yoghurt & 13.9 & 13.9 & 0.0 & 0.0 & 13.9 & 0.0 & 13.9 & 0.0 & 13.9 & 13.9 & 0.0 & 0.0 & 13.9 & 13.9 & 13.9 & 13.9 \\
\hline Starchy & 20.0 & 30.0 & 40.0 & 40.0 & 50.1 & 50.1 & 60.1 & 50.1 & 20.0 & 30.0 & 40.0 & 40.0 & 40.0 & 40.0 & 40.0 & 40.0 \\
\hline Eggs & 19.0 & 19.0 & 19.0 & 19.0 & 28.5 & 33.2 & 33.2 & 33.2 & 14.2 & 14.2 & 19.0 & 19.0 & 19.0 & 23.7 & 23.7 & 23.7 \\
\hline $\begin{array}{l}\text { Trassport } \\
\text { Trans }\end{array}$ & 72.5 & 75.7 & 82.6 & 88.7 & 86.2 & 89.4 & 88.3 & 80.4 & 70.9 & 71.7 & 74.6 & 67.1 & 70.2 & 70.6 & 73.1 & 69.7 \\
\hline Total & 1784.4 & 2004.8 & 2296.2 & 2636.2 & 2617.9 & 2729.5 & 2561.7 & 2222.7 & 1719.0 & 1906.4 & 2030.3 & 1825.6 & 1955.0 & 1950.8 & 1919.7 & 1805.3 \\
\hline
\end{tabular}

\begin{tabular}{|c|c|c|c|c|c|c|c|c|c|c|c|c|c|c|c|c|}
\hline \multirow{3}{*}{ Nutritional Category } & \multicolumn{8}{|l|}{ Males } & \multicolumn{8}{|l|}{ Females } \\
\hline & \multirow{2}{*}{\multicolumn{8}{|c|}{ Age }} & \\
\hline & & $4-8$ & $9-13$ & $14-18$ & $19-30$ & $31-50$ & $51-70$ & $71+$ & $1-3$ & $4-8$ & $9-13$ & $14-18$ & $19-30$ & $31-50$ & $51-70$ & $71+$ \\
\hline Poultry & 0.000301 & 0.000368 & 0.000501 & 0.000602 & 0.000735 & 0.000635 & 0.000602 & 0.000368 & 0.000267 & 0.000334 & 0.000401 & 0.000535 & 0.000501 & 0.000468 & 0.000435 & 0.000334 \\
\hline Citrus & 0.000156 & 0.000156 & 0.000156 & 0.000156 & $7.80 \mathrm{E}-05$ & 0.000156 & 0.000234 & 0.000234 & 0.000156 & 0.000156 & 0.000156 & $7.80 \mathrm{E}-05$ & 0.000156 & 0.000156 & 0.000234 & 0.000234 \\
\hline Fish and Seafood & $1.34 \mathrm{E}-05$ & $1.34 \mathrm{E}-05$ & $2.67 \mathrm{E}-05$ & $4.01 \mathrm{E}-05$ & $8.02 \mathrm{E}-05$ & $9.36 \mathrm{E}-05$ & 0.000107 & $8.02 \mathrm{E}-05$ & $1.34 \mathrm{E}-05$ & $2.67 \mathrm{E}-05$ & $2.67 \mathrm{E}-05$ & $2.67 \mathrm{E}-05$ & $5.35 \mathrm{E}-05$ & $6.68 \mathrm{E}-05$ & $8.02 \mathrm{E}-05$ & $6.68 \mathrm{E}-05$ \\
\hline Other Fruits & 0.000529 & 0.000529 & 0.000441 & 0.000353 & 0.000353 & 0.000441 & 0.000529 & 0.000617 & 0.000529 & 0.000441 & 0.000441 & 0.000353 & 0.000353 & 0.000441 & 0.000617 & 0.000617 \\
\hline Meat & & & & & 0.00 & 0.00 & 0.00 & 0.00 & 0.000923 & 0.001426 & 0.001678 & 0.001342 & & 0.001762 & 0.001594 & 0.00151 \\
\hline Grains & 0.000666 & 0.000991 & 0.001186 & 0.001332 & 0.001316 & 0.001267 & 0.001121 & 0.000975 & 0.000601 & 0.00091 & 0.001056 & 0.000991 & 0.000958 & 0.000893 & 0.000829 & 0.000796 \\
\hline Dark Greens & 0 & 0 & 0 & $2.09 \mathrm{E}-05$ & $2.09 \mathrm{E}-05$ & $2.09 \mathrm{E}-05$ & $4.19 \mathrm{E}-05$ & $2.09 \mathrm{E}-05$ & 0 & 0 & 0 & $2.09 \mathrm{E}-05$ & $2.09 \mathrm{E}-05$ & $2.09 \mathrm{E}-05$ & $4.19 \mathrm{E}-05$ & $2.09 \mathrm{E}-05$ \\
\hline Red and Orange & $6.31 \mathrm{E}-05$ & $9.47 \mathrm{E}-05$ & $9.47 \mathrm{E}-05$ & 0.000126 & 0.000158 & 0.000158 & 0.000126 & 0.000126 & $6.31 \mathrm{E}-05$ & $6.31 \mathrm{E}-05$ & $9.47 \mathrm{E}-05$ & $9.47 \mathrm{E}-05$ & 0.000126 & 0.000126 & 0.000126 & 0.000126 \\
\hline Sugars & $1.79 \mathrm{E}-05$ & $2.99 \mathrm{E}-05$ & $4.10 \mathrm{E}-05$ & $4.69 \mathrm{E}-05$ & $4.48 \mathrm{E}-05$ & $3.91 \mathrm{E}-05$ & $3.14 \mathrm{E}-05$ & $2.67 \mathrm{E}-05$ & $1.60 \mathrm{E}-05$ & $2.72 \mathrm{E}-05$ & $3.39 \mathrm{E}-05$ & $3.33 \mathrm{E}-05$ & $3.18 \mathrm{E}-05$ & $2.88 \mathrm{E}-05$ & $2.38 \mathrm{E}-05$ & $2.08 \mathrm{E}-05$ \\
\hline Nuts & $3.04 \mathrm{E}-05$ & $4.06 \mathrm{E}-1$ & 5.07E- 05 & 4.06E-05 & 5.07E-05 & $8.12 \mathrm{E}-05$ & 9.13E-05 & 7.10E-05 & $2.03 \mathrm{E}-05$ & $3.04 \mathrm{E}-05$ & $4.06 \mathrm{E}-05$ & $3.04 \mathrm{E}-05$ & $4.06 \mathrm{E}-05$ & $6.09 \mathrm{E}-05$ & 7.10E-05 & 5.07E- 05 \\
\hline Milk & 0.001767 & 0.001395 & 0.001488 & 0.001395 & 0.000744 & 0.000837 & 0.000837 & 0.001023 & 0.001767 & 0.001395 & 0.001209 & 0.000837 & 0.000651 & 0.000744 & 0.000744 & 0.000837 \\
\hline Cheese & 0.00026 & 0.00039 & 0.00052 & 0.00065 & 0.00065 & 0.000585 & 0.000455 & 0.00026 & 0.00026 & 0.00039 & 0.00039 & 0.000455 & 0.000455 & 0.00039 & 0.000325 & 0.000195 \\
\hline Juice & 0.000555 & 0.000396 & 0.000317 & 0.000317 & 0.000317 & 0.000238 & 0.000238 & 0.000317 & 0.000555 & 0.000317 & 0.000317 & 0.000238 & 0.000317 & 0.000158 & 0.000238 & 0.000317 \\
\hline Beverages & 0.000934 & 0.000934 & 0.000934 & 0.000934 & 0.000934 & 0.000934 & 0.000934 & 0.000934 & 0.000934 & 0.000934 & 0.000934 & 0.000934 & 0.000934 & 0.000934 & 0.000934 & 0.000934 \\
\hline
\end{tabular}




\begin{tabular}{|c|c|c|c|c|c|c|c|c|c|c|c|c|c|c|c|c|}
\hline Fats and Oils & 0.000742 & 0.000951 & 0.001124 & 0.001291 & 0.001276 & 0.001369 & 0.001261 & 0.001054 & 0.000695 & 0.000892 & 0.001014 & 0.00097 & 0.000964 & 0.000955 & 0.000955 & 0.000837 \\
\hline Legumes and Soy & 1.40E-05 & $1.40 \mathrm{E}-05$ & $2.09 \mathrm{E}-05$ & $2.79 \mathrm{E}-05$ & $4.19 \mathrm{E}-05$ & $4.88 \mathrm{E}-05$ & $4.19 \mathrm{E}-05$ & $2.79 \mathrm{E}-05$ & $1.40 \mathrm{E}-05$ & $1.40 \mathrm{E}-05$ & $2.09 \mathrm{E}-05$ & $2.09 \mathrm{E}-05$ & $2.79 \mathrm{E}-05$ & 3.49E-05 & $3.49 \mathrm{E}-05$ & $2.09 \mathrm{E}-05$ \\
\hline Other Vegetables & $4.26 \mathrm{E}-05$ & $8.51 \mathrm{E}-05$ & $8.51 \mathrm{E}-05$ & 0.000128 & 0.000255 & 0.000255 & 0.000298 & 0.000213 & $8.51 \mathrm{E}-05$ & $8.51 \mathrm{E}-05$ & 0.000128 & 0.000128 & 0.000213 & 0.000255 & 0.000298 & 0.000213 \\
\hline Yoghurt & $6.24 \mathrm{E}-05$ & $6.24 \mathrm{E}-05$ & 0 & 0 & $6.24 \mathrm{E}-05$ & 0 & $6.24 \mathrm{E}-05$ & 0 & $6.24 \mathrm{E}-05$ & $6.24 \mathrm{E}-05$ & 0 & 0 & $6.24 \mathrm{E}-05$ & $6.24 \mathrm{E}-05$ & $6.24 \mathrm{E}-05$ & $6.24 \mathrm{E}-05$ \\
\hline Starchy & 0.000177 & 0.000265 & 0.000353 & 0.000353 & 0.000442 & 0.000442 & 0.00053 & 0.000442 & 0.000177 & 0.000265 & 0.000353 & 0.000353 & 0.000353 & 0.000353 & 0.000353 & 0.000353 \\
\hline Eggs & $8.23 \mathrm{E}-05$ & $8.23 \mathrm{E}-05$ & $8.23 \mathrm{E}-05$ & $8.23 \mathrm{E}-05$ & 0.000123 & 0.000144 & 0.000144 & 0.000144 & $6.17 \mathrm{E}-05$ & $6.17 \mathrm{E}-05$ & $8.23 \mathrm{E}-05$ & $8.23 \mathrm{E}-05$ & $8.23 \mathrm{E}-05$ & 0.000103 & 0.000103 & 0.000103 \\
\hline Transport & $2.67 \mathrm{E}-05$ & $2.76 \mathrm{E}-05$ & $2.99 \mathrm{E}-05$ & $3.22 \mathrm{E}-05$ & $3.11 \mathrm{E}-05$ & $3.18 \mathrm{E}-05$ & $3.14 \mathrm{E}-05$ & $2.88 \mathrm{E}-05$ & $2.62 \mathrm{E}-05$ & $2.62 \mathrm{E}-05$ & $2.71 \mathrm{E}-05$ & $2.45 \mathrm{E}-05$ & $2.55 \mathrm{E}-05$ & $2.54 \mathrm{E}-05$ & $2.63 \mathrm{E}-05$ & $2.53 \mathrm{E}$ \\
\hline Total & 0.007446 & 0.008335 & 0.009382 & 0.010613 & 0.010566 & 0.010964 & 0.010484 & 0.009227 & 0.007226 & 0.007858 & 0.008403 & 0.007548 & 0.008089 & 0.00804 & 0.008125 & 0.007675 \\
\hline
\end{tabular}

Census data are taken from American Fact Finder at the block-group level ${ }^{7}$. These data provide population based on sex and age group. The age groups in the census data do not precisely align with those in NHANES, so concordance was made based on best judgement, as shown in Table S29. Census data is also adjusted for incarcerated population since their usual daily intakes are likely not well represented by NHANES. This means subtracting 1418 adults (taken from age groups based on proportion of unaltered population) from block group '250250801001' as it contains the Suffolk County Correctional Facility ${ }^{8}$.

\begin{tabular}{|c|c|}
\hline NHANES age group & Census age groups \\
\hline $1-3$ & 'under 5 years' \\
\hline $4-8$ & '5 to 9 years' \\
\hline $9-13$ & '10 to 14 years' \\
\hline $14-18$ & '15 to 17 years', ' 18 and 19 years' \\
\hline $19-30$ & '20 years', '21 years', '22-24 years', '25-29 years' \\
\hline $31-50$ & '30-34 years', '35-39 years', '40-44 years', '45-49 years' \\
\hline $51-70$ & '50-54 years', '55-59 years', ' 60 and 61 years', ' 62 to 64 years', ' 65 and 66 years', ' 67 to 69 years' \\
\hline $71+$ & '70 to 74 years', ' 75 to 79 years', ' 80 to 84 years', ' $85+$ years' \\
\hline
\end{tabular}

With the block-group demographics data in hand and estimated environmental burdens for the different age groups and sexes, Boston's food related environmental impacts are calculated for the 560 block-groups that comprise the city. Figures 1-2 show the estimated GWP impacts and land use for Boston's food consumption for the year 2010.

Figure S1. 2010 food-borne GHG emissions

for Boston block-groups

(white indicates uninhabited

block groups devoid of impacts)

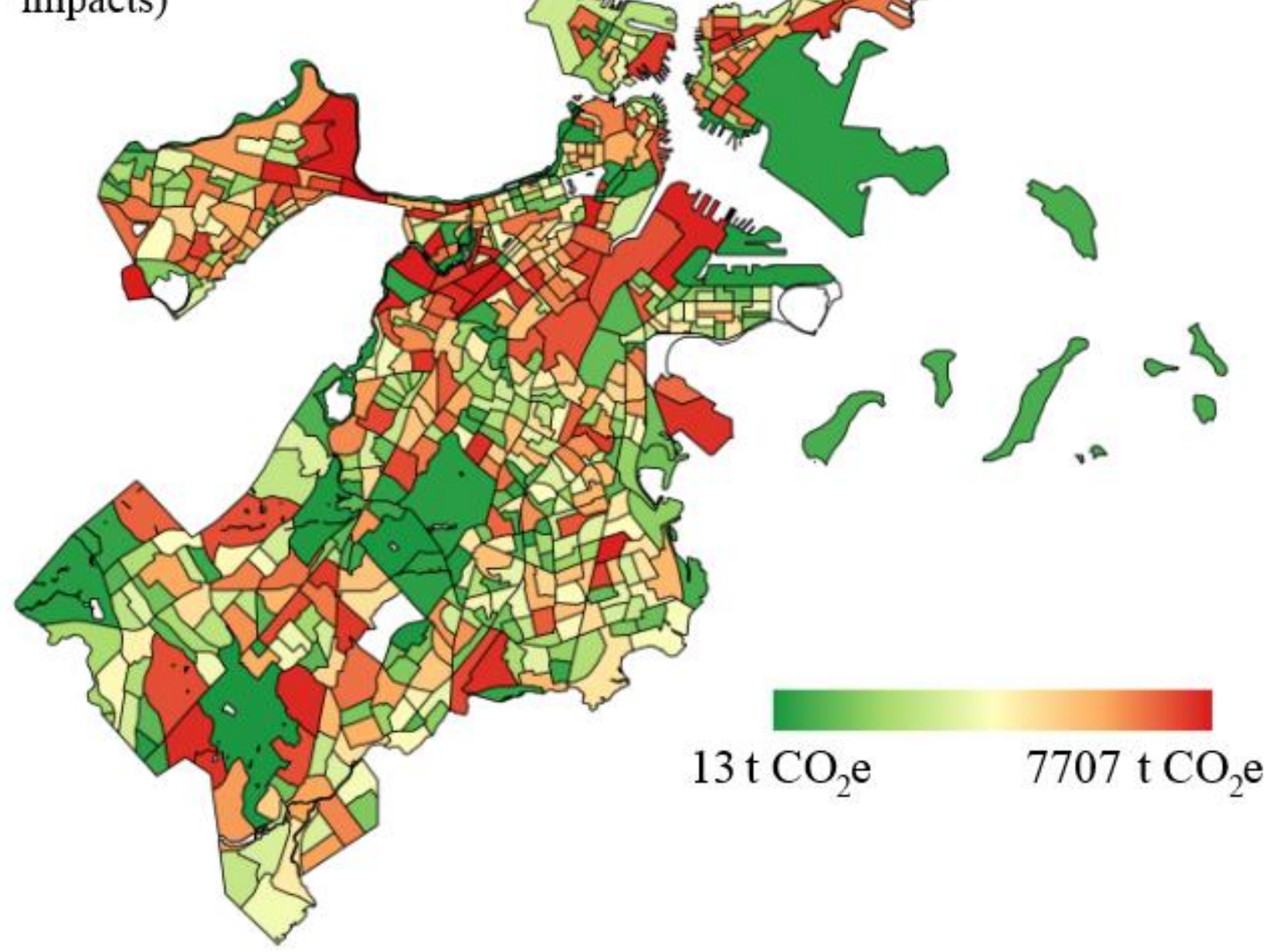


Figure S2. 2010 food-borne land use for Boston block-groups (white indicates uninhabited block-groups devoid of impacts)

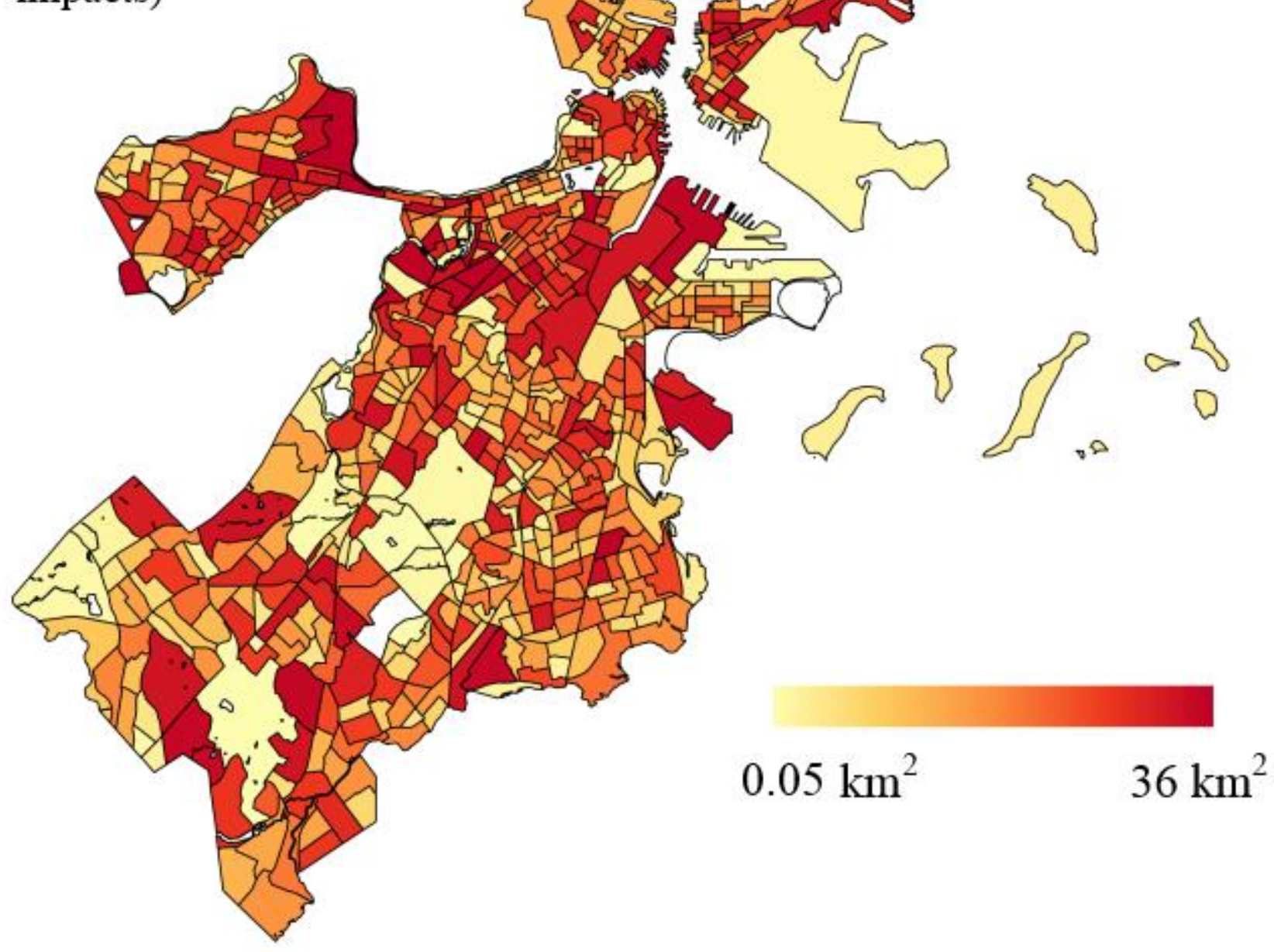

\section{Life Cycle Inventories and LCA for Urban Agriculture}

Life cycle inventories (LCI) for the urban farms build upon those from an earlier study of farms growing tomato and lettuce in Boston in New York City ${ }^{9}$. Of the six farms covering five UF forms from the earlier study, only three of the farms and two farm-types are used in this study, for a number of reasons:

- They produced the widest variety of crops, useful when modelling city-wide impacts of UF (difficult to model a city only consuming tomatoes)

- They represent the predominant UF forms in the study region at the time of publishing: open plots and rooftop farms (see Figure 3 for examples of each). See Goldstein et al. (2016) ${ }^{10}$ for more information about the nuances between UF types and their divergent environmental performance.

○ Open plots typically low-tech operations, growing crops directly in local overburden or raised beds

- Rooftop farms are identical in most respects to green roofs with the exception that they grow edible crops. Soil depth is typically equal to greater than 12 ", and hence, rooftop farms qualify as intensive green roofs.

- Have superior environmental performance than conventional agriculture for some foods and by some metrics, as opposed to the other forms which had higher environmental intensities compared to conventional UF'. Although this skews the results in UF's favor, it is useful in a hypothetical study of large scale urban design to quantify the potential best-case, hypothetical benefits of UF. Additionally, since UF is not universally preferable to conventional produce, this will still provide opportunities to discuss trade-offs when adapting UF.

Figure 3 - Open rooftop farm (left) and open lot farm (right). Authors own photographs.

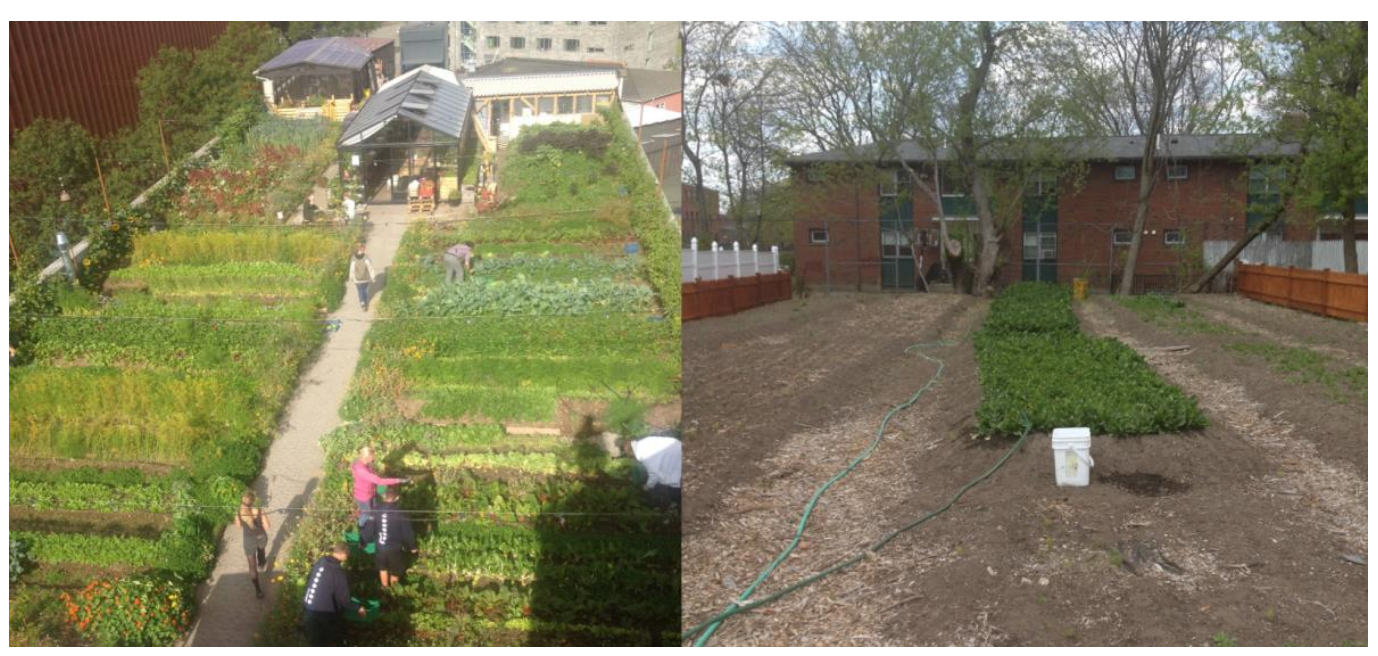

The attributes of the utilized UF systems are outline in Table S30.

\begin{tabular}{|l|l|l|l|l|l|}
\hline Table S30. Urban farm characteristics and crops \\
\hline Location & Farm & Farm Type & Area $\left(\mathbf{m}^{2}\right)$ & For profit? & Crops \\
\hline Boston, MA & 1 & open plot & 560 & No & tomato, bell pepper, eggplant, lettuce* \\
\hline Boston, MA & 2 & open rooftop & 1469 & Yes & $\begin{array}{l}\text { turnip, tomato, scallion, radish, bell pepper, lettuce, kale, } \\
\text { cucumber, carrot, green bean }\end{array}$ \\
\hline New York City & 3 & open plot & 1269 & No & $\begin{array}{l}\text { turnip, tomato, squash, scallions, bell pepper, lettuce, kale, } \\
\text { cucumber, collard greens, carrot, cabbage, beet, green bean }\end{array}$ \\
\hline
\end{tabular}
* Technically 'arugula' but assumed lettuce here since it performs the same function as lettuce (salad greens, sandwich topping, etc.)

Technically 'arugula' but assumed lettuce here since it performs the same function as lettuce (salad greens, sandwich topping, etc.) 
Process-based LCA methodology is applied here. The LCA scope is production of crops and distribution to final consumers - in line with the MRIO model used to assess city-wide impacts. Where by-products occur, system expansion is applied to credit the urban farm in accordance with the ISO 14040 family ${ }^{11}$. The ecoinvent database version 3.2 was used to provide data on background processes and to perform the life cycle impact assessment for the different foods. Primary data was collected over the 2015 growing season. Tables S31-33 outline the Life Cycle Inventories to produce 1 kilogram of different crops from the modeled farms.

\begin{tabular}{|c|c|c|c|c|c|}
\hline & Unit & Tomato & Bell Pepper & Eggplant & Arugula \\
\hline \multirow{2}{*}{\multicolumn{6}{|c|}{$\begin{array}{l}\text { Materials and Energy Inputs } \\
\text { Capital }\end{array}$}} \\
\hline & & & \multicolumn{3}{|c|}{ Capital } \\
\hline Concrete, normal $\{$ US-NPCC $\} \mid$ production $\mid$ Conseq, $\mathrm{U}$ & $\mathrm{m}^{3}$ & $2.07 \mathrm{E}-05$ & $3.64 \mathrm{E}-05$ & $3.35 \mathrm{E}-05$ & $1.25 \mathrm{E}-04$ \\
\hline Extrusion, plastic film \{US-NPCC $\} \mid$ production $\mid$ Conseq, $U$ & $\mathrm{~kg}$ & $1.24 \mathrm{E}-02$ & $2.19 \mathrm{E}-02$ & $2.02 \mathrm{E}-02$ & $7.50 \mathrm{E}-02$ \\
\hline Occupation, urban, continuously built & $\mathrm{m}^{2}$ & $2.53 \mathrm{E}-01$ & $4.46 \mathrm{E}-01$ & $4.10 \mathrm{E}-01$ & $1.52 \mathrm{E}+00$ \\
\hline Polyethylene, high density, granulate \{GLO\}| market for |Conseq, U & $\mathrm{kg}$ & $1.17 \mathrm{E}-03$ & $2.06 \mathrm{E}-03$ & $1.89 \mathrm{E}-03$ & $7.04 \mathrm{E}-03$ \\
\hline Sawnwood, hardwood, air dried, planed $\{$ RoW $\} \mid$ market for $\mid$ Conseq, U & $\mathrm{m}^{3}$ & $4.08 \mathrm{E}-05$ & $7.19 \mathrm{E}-05$ & $6.61 \mathrm{E}-05$ & $2.46 \mathrm{E}-04$ \\
\hline Steel, low-alloyed, hot rolled \{US-NPCC $\} \mid$ market for | Conseq, U & $\mathrm{kg}$ & $6.76 \mathrm{E}-04$ & $1.19 \mathrm{E}-03$ & $1.09 \mathrm{E}-03$ & $4.08 \mathrm{E}-03$ \\
\hline Synthetic rubber $\{$ GLO $\} \mid$ market for $\mid$ Conseq, U & $\mathrm{kg}$ & $8.35 \mathrm{E}-04$ & $1.47 \mathrm{E}-03$ & $1.35 \mathrm{E}-03$ & $5.04 \mathrm{E}-03$ \\
\hline Transport, freight, lorry >32 metric ton, EURO4 \{GLO\}| market for $\mid$ Conseq, U & $\mathrm{tkm}$ & $1.70 \mathrm{E}-01$ & $3.00 \mathrm{E}-01$ & $2.76 \mathrm{E}-01$ & $1.03 \mathrm{E}+00$ \\
\hline Wood chips, wet, measured as dry mass $\{$ RoW $\} \mid$ market for $\mid$ Conseq, $\mathrm{U}$ & $\mathrm{m}^{3}$ & $1.85 \mathrm{E}-01$ & $3.26 \mathrm{E}-01$ & $2.99 \mathrm{E}-01$ & $1.11 \mathrm{E}+00$ \\
\hline \multicolumn{6}{|l|}{ Operations } \\
\hline Polyethylene, low density, granulate $\{\mathrm{GLO}\} \mid$ market for $\mid$ Conseq, $\mathrm{U}$ & $\mathrm{kg}$ & $3.49 \mathrm{E}-03$ & $6.15 \mathrm{E}-03$ & $5.66 \mathrm{E}-03$ & $2.11 \mathrm{E}-02$ \\
\hline Polypropylene, granulate $\{\mathrm{GLO}\} \mid$ market for $\mid$ Conseq, $\mathrm{U}$ & $\mathrm{kg}$ & $6.95 \mathrm{E}-03$ & $1.23 \mathrm{E}-02$ & $1.13 \mathrm{E}-02$ & $4.19 \mathrm{E}-02$ \\
\hline Tap water \{US-Boston\}| market for |Conseq, U & $\mathrm{m}^{3}$ & $7.77 \mathrm{E}-02$ & $1.31 \mathrm{E}-01$ & $1.02 \mathrm{E}-01$ & $1.28 \mathrm{E}-01$ \\
\hline $\begin{array}{l}\text { Transport, passenger car, large size, petrol, EURO } 4 \text { \{RER }\} \mid \text { transport, passenger car, } \\
\text { large size, petrol, EURO } 4 \text { | Conseq, U }\end{array}$ & $\mathrm{km}$ & $4.91 \mathrm{E}-02$ & $8.66 \mathrm{E}-02$ & $7.96 \mathrm{E}-02$ & $2.96 \mathrm{E}-01$ \\
\hline \multicolumn{6}{|l|}{ Waste } \\
\hline Inert waste, for final disposal $\{\mathrm{GLO}\} \mid$ market for $\mid$ Conseq, $\mathrm{U}$ & $\mathrm{kg}$ & $1.27 \mathrm{E}-04$ & $2.24 \mathrm{E}-04$ & $2.06 \mathrm{E}-04$ & $7.65 \mathrm{E}-04$ \\
\hline Inert waste, for final disposal $\{$ US $\} \mid$ market for $\mid$ Conseq, $U$ & $\mathrm{~kg}$ & $1.36 \mathrm{E}-02$ & $2.39 \mathrm{E}-02$ & $2.20 \mathrm{E}-02$ & $8.19 \mathrm{E}-02$ \\
\hline $\mathrm{PE}$ (waste treatment) $\{\mathrm{US}-\mathrm{NPCC}\} \mid$ recycling of PE $\mid$ Conseq, $\mathrm{U}$ & $\mathrm{kg}$ & $3.64 \mathrm{E}-03$ & $6.41 \mathrm{E}-03$ & $5.89 \mathrm{E}-03$ & $2.19 \mathrm{E}-02$ \\
\hline Rubber (waste treatment) $\{$ US-NPCC $\} \mid$ recycling of rubber $\mid$ Conseq, $U$ & $\mathrm{~kg}$ & $7.73 \mathrm{E}-04$ & $1.36 \mathrm{E}-03$ & $1.25 \mathrm{E}-03$ & $4.66 \mathrm{E}-03$ \\
\hline Steel and iron (waste treatment) \{US-NPCC $\} \mid$ recycling of steel and iron $\mid$ Conseq, $\mathrm{U}$ & $\mathrm{kg}$ & $3.38 \mathrm{E}-04$ & $5.96 \mathrm{E}-04$ & $5.47 \mathrm{E}-04$ & $2.04 \mathrm{E}-03$ \\
\hline Waste concrete gravel \{US-NPCC $\} \mid$ treatment of, recycling | Conseq, U & $\mathrm{kg}$ & $4.57 \mathrm{E}-02$ & $8.05 \mathrm{E}-02$ & $7.40 \mathrm{E}-02$ & $2.75 \mathrm{E}-01$ \\
\hline Waste wood, post-consumer $\{\mathrm{GLO}\} \mid$ market for $\mid$ Conseq, $\mathrm{U}$ & $\mathrm{kg}$ & $1.30 \mathrm{E}-02$ & $2.29 \mathrm{E}-02$ & $2.10 \mathrm{E}-02$ & $7.83 \mathrm{E}-02$ \\
\hline
\end{tabular}

\begin{tabular}{|c|c|c|c|c|c|c|}
\hline & Unit & Turnip & Tomato & Scallion & Radish & Bell Pepper \\
\hline \multicolumn{7}{|l|}{ Materials and Energy Inputs } \\
\hline \multicolumn{7}{|l|}{ Capital } \\
\hline Aluminium, primary, ingot $\{\mathrm{US}\} \mid$ market for $\mid$ Conseq, $\mathrm{U}$ & $\mathrm{kg}$ & $2.0 \mathrm{E}-06$ & $7.8 \mathrm{E}-07$ & $3.9 \mathrm{E}-06$ & $1.2 \mathrm{E}-06$ & $1.5 \mathrm{E}-06$ \\
\hline Copper $\{\mathrm{GLO}\} \mid$ market for $\mid$ Conseq, $\mathrm{U}$ & $\mathrm{kg}$ & $6.4 \mathrm{E}-06$ & $2.5 \mathrm{E}-06$ & $1.3 \mathrm{E}-05$ & $3.8 \mathrm{E}-06$ & $4.9 \mathrm{E}-06$ \\
\hline Crushed gravel \{US-Boston\} $\mid$ market for $\mid$ conseq, $\mathrm{U}$ & $\mathrm{kg}$ & $3.2 \mathrm{E}-01$ & $1.2 \mathrm{E}-01$ & $6.3 \mathrm{E}-01$ & $1.9 \mathrm{E}-01$ & $2.4 \mathrm{E}-01$ \\
\hline Expanded clay $\{$ US-Boston $\} \mid$ Market for $\mid$ Conseq, $U$ & $\mathrm{~kg}$ & $2.0 \mathrm{E}+00$ & $7.7 \mathrm{E}-01$ & $3.9 \mathrm{E}+00$ & $1.2 \mathrm{E}+00$ & $1.5 \mathrm{E}+00$ \\
\hline Expanded shale \{US-Boston\} $\mid$ Market for $\mid$ Conseq, U & $\mathrm{kg}$ & $1.8 \mathrm{E}-01$ & $7.1 \mathrm{E}-02$ & $3.6 \mathrm{E}-01$ & $1.1 \mathrm{E}-01$ & $1.4 \mathrm{E}-01$ \\
\hline Extrusion, plastic film $\{$ US-MRO\}| production $\mid$ Conseq, $\mathrm{U}$ & $\mathrm{kg}$ & $4.1 \mathrm{E}-02$ & $1.6 \mathrm{E}-02$ & $8.1 \mathrm{E}-02$ & $2.4 \mathrm{E}-02$ & $3.1 \mathrm{E}-02$ \\
\hline Extrusion, plastic film $\{$ US-NPCC $\} \mid$ production $\mid$ Conseq, $U$ & $\mathrm{~kg}$ & $2.4 \mathrm{E}-02$ & $9.3 \mathrm{E}-03$ & $4.7 \mathrm{E}-02$ & $1.4 \mathrm{E}-02$ & $1.8 \mathrm{E}-02$ \\
\hline Extrusion, plastic pipes \{US-NPCC $\}$ production $\mid$ Conseq, $\mathrm{U}$ & $\mathrm{kg}$ & $1.0 \mathrm{E}-03$ & $4.1 \mathrm{E}-04$ & $2.1 \mathrm{E}-03$ & $6.1 \mathrm{E}-04$ & $7.9 \mathrm{E}-04$ \\
\hline $\begin{array}{l}\text { Glass, for liquid crystal display }\{\mathrm{GLO}\} \mid \text { production } \mid \text { Conseq, } \mathrm{U} \\
\end{array}$ & $\mathrm{kg}$ & $1.4 \mathrm{E}-07$ & $5.6 \mathrm{E}-08$ & $2.8 \mathrm{E}-07$ & $8.4 \mathrm{E}-08$ & $1.1 \mathrm{E}-07$ \\
\hline Nylon $6\{\mathrm{GLO}\} \mid$ market for $\mid$ Conseq, $\mathrm{U}$ & $\mathrm{kg}$ & $1.1 \mathrm{E}-05$ & $4.1 \mathrm{E}-06$ & $2.1 \mathrm{E}-05$ & $6.2 \mathrm{E}-06$ & $7.9 \mathrm{E}-06$ \\
\hline Polyethylene, high density, granulate $\{\mathrm{GLO}\} \mid$ market for $\mid$ Conseq, $\mathrm{U}$ & $\mathrm{kg}$ & $3.3 \mathrm{E}-02$ & $1.3 \mathrm{E}-02$ & $6.5 \mathrm{E}-02$ & $1.9 \mathrm{E}-02$ & $2.5 \mathrm{E}-02$ \\
\hline Polypropylene, granulate $\{\mathrm{GLO}\} \mid$ market for $\mid$ Conseq, $\mathrm{U}$ & $\mathrm{kg}$ & $1.1 \mathrm{E}-02$ & $4.2 \mathrm{E}-03$ & $2.1 \mathrm{E}-02$ & $6.4 \mathrm{E}-03$ & $8.2 \mathrm{E}-03$ \\
\hline Steel, low-alloyed, hot rolled $\{$ US-MRO $\} \mid$ market for $\mid$ Conseq, U & $\mathrm{kg}$ & $1.7 \mathrm{E}-03$ & $6.5 \mathrm{E}-04$ & $3.3 \mathrm{E}-03$ & $9.8 \mathrm{E}-04$ & $1.2 \mathrm{E}-03$ \\
\hline Steel, low-alloyed, hot rolled $\{$ US-NPCC $\} \mid$ market for $\mid$ Conseq, U & $\mathrm{kg}$ & $4.8 \mathrm{E}-01$ & $1.9 \mathrm{E}-01$ & $9.6 \mathrm{E}-01$ & $2.9 \mathrm{E}-01$ & $3.7 \mathrm{E}-01$ \\
\hline Steel, low-alloyed, hot rolled $\{$ US-WECC $\} \mid$ market for $\mid$ Conseq, U & $\mathrm{kg}$ & $9.7 \mathrm{E}-05$ & $3.8 \mathrm{E}-05$ & $1.9 \mathrm{E}-04$ & $5.7 \mathrm{E}-05$ & $7.3 \mathrm{E}-05$ \\
\hline Transport, freight, lorry $>32$ metric ton, EURO4 $\{\mathrm{GLO}\} \mid$ market for $\mid$ Conseq, $\mathrm{U}$ & $\mathrm{tkm}$ & $1.1 \mathrm{E}-01$ & $4.3 \mathrm{E}-02$ & $2.2 \mathrm{E}-01$ & $6.5 \mathrm{E}-02$ & $8.3 \mathrm{E}-02$ \\
\hline Transport, freight, lorry 16-32 metric ton, EURO4 $\{\mathrm{GLO}\} \mid$ market for $\mid$ Conseq, $\mathrm{U}$ & $\mathrm{tkm}$ & $1.9 \mathrm{E}-02$ & $7.5 \mathrm{E}-03$ & $3.8 \mathrm{E}-02$ & $1.1 \mathrm{E}-02$ & $1.4 \mathrm{E}-02$ \\
\hline Wire drawing, copper $\{$ US-WECC $\} \mid$ processing $\mid$ Conseq, $U$ & $\mathrm{~kg}$ & $6.4 \mathrm{E}-06$ & $2.5 \mathrm{E}-06$ & $1.3 \mathrm{E}-05$ & $3.8 \mathrm{E}-06$ & $4.9 \mathrm{E}-06$ \\
\hline \multicolumn{7}{|l|}{ Operations } \\
\hline Phosphate fertiliser, as P2O5 \{GLO $\} \mid$ market for $\mid$ Conseq, U & $\mathrm{kg}$ & $1.2 \mathrm{E}-03$ & $4.7 \mathrm{E}-04$ & $2.4 \mathrm{E}-03$ & $7.1 \mathrm{E}-04$ & $9.1 \mathrm{E}-04$ \\
\hline Ammonium nitrate, as $\mathrm{N}\{\mathrm{RER}\} \mid$ ammonium nitrate production $\mid$ Conseq, $\mathrm{U}$ & $\mathrm{kg}$ & $9.4 \mathrm{E}-04$ & $3.7 \mathrm{E}-04$ & $1.9 \mathrm{E}-03$ & $5.5 \mathrm{E}-04$ & $7.1 \mathrm{E}-04$ \\
\hline Potassium nitrate $\{\mathrm{GLO}\} \mid$ market for $\mid$ Conseq, $\mathrm{U}$ & $\mathrm{kg}$ & $6.5 \mathrm{E}-04$ & $2.5 \mathrm{E}-04$ & $1.3 \mathrm{E}-03$ & $3.8 \mathrm{E}-04$ & $4.9 \mathrm{E}-04$ \\
\hline Transport, passenger car, small size, petrol, EURO 5 \{GLO\}| market for | Conseq, U & $\mathrm{km}$ & $1.2 \mathrm{E}+00$ & $4.7 \mathrm{E}-01$ & $2.4 \mathrm{E}+00$ & $7.1 \mathrm{E}-01$ & $9.1 \mathrm{E}-01$ \\
\hline Electricity, low voltage, $2012-2040$ average $\{$ NPCC, US only $\} \mid$ market for $\mid$ Conseq, $U$ & MJ & $6.0 \mathrm{E}-02$ & $2.3 \mathrm{E}-02$ & $1.2 \mathrm{E}-01$ & $3.5 \mathrm{E}-02$ & $4.5 \mathrm{E}-02$ \\
\hline Basalt $\{\mathrm{GLO}\} \mid$ market for $\mid$ Conseq, $\mathrm{U}$ & $\mathrm{kg}$ & $5.0 \mathrm{E}-02$ & $2.0 \mathrm{E}-02$ & $1.0 \mathrm{E}-01$ & $3.0 \mathrm{E}-02$ & $3.8 \mathrm{E}-02$ \\
\hline compost $\{$ US-NPCC $\} \mid$ at farm $\mid$ conseq, $U$ & $\mathrm{~kg}$ & $4.3 \mathrm{E}-01$ & $1.7 \mathrm{E}-01$ & $8.4 \mathrm{E}-01$ & $2.5 \mathrm{E}-01$ & $3.2 \mathrm{E}-01$ \\
\hline garden waste treatment $\{$ US-NPCC $\} \mid$ at farm $\mid$ conseq, $U$ & $\mathrm{~kg}$ & $1.3 \mathrm{E}-01$ & $4.9 \mathrm{E}-02$ & $2.5 \mathrm{E}-01$ & $7.5 \mathrm{E}-02$ & $9.5 \mathrm{E}-02$ \\
\hline Tap water $\{$ US-Boston\} $\mid$ market for $\mid$ Conseq, $\mathrm{U}$ & $\mathrm{m}^{3}$ & $1.4 \mathrm{E}-02$ & $5.6 \mathrm{E}-03$ & $2.9 \mathrm{E}-02$ & $8.5 \mathrm{E}-03$ & $1.1 \mathrm{E}-02$ \\
\hline \multicolumn{7}{|l|}{ Waste } \\
\hline Aluminium (waste treatment) $\{$ US-NPCC $\} \mid$ recycling of aluminium $\mid$ Conseq, $U$ & $\mathrm{~kg}$ & $8.9 \mathrm{E}-07$ & $3.5 \mathrm{E}-07$ & $1.8 \mathrm{E}-06$ & $5.3 \mathrm{E}-07$ & $6.8 \mathrm{E}-07$ \\
\hline Copper (waste treatment) \{US-NPCC $\} \mid$ recycling of copper $\mid$ Conseq, $\mathrm{U}$ & $\mathrm{kg}$ & $8.9 \mathrm{E}-07$ & $3.5 \mathrm{E}-07$ & $1.8 \mathrm{E}-06$ & $5.3 \mathrm{E}-07$ & $6.8 \mathrm{E}-07$ \\
\hline Inert waste, for final disposal $\{$ US $\} \mid$ market for $\mid$ Conseq, $U$ & $\mathrm{~kg}$ & $2.5 \mathrm{E}+00$ & $9.7 \mathrm{E}-01$ & $4.9 \mathrm{E}+00$ & $1.5 \mathrm{E}+00$ & $1.9 \mathrm{E}+00$ \\
\hline PE (waste treatment) \{US-NPCC $\} \mid$ recycling of PE $\mid$ Conseq, U & $\mathrm{kg}$ & $5.2 \mathrm{E}-02$ & $2.0 \mathrm{E}-02$ & $1.0 \mathrm{E}-01$ & $3.1 \mathrm{E}-02$ & $3.9 \mathrm{E}-02$ \\
\hline $\mathrm{PP}($ waste treatment) \{US-NPCC$\} \mid$ recycling of PP $\mid$ Conseq, $\mathrm{U}$ & $\mathrm{kg}$ & $1.0 \mathrm{E}-02$ & $4.0 \mathrm{E}-03$ & $2.0 \mathrm{E}-02$ & $6.0 \mathrm{E}-03$ & $7.7 \mathrm{E}-03$ \\
\hline Steel and iron (waste treatment) $\{$ US-NPCC $\} \mid$ recycling of steel and iron $\mid$ Conseq, $U$ & $\mathrm{~kg}$ & $4.7 \mathrm{E}-01$ & $1.9 \mathrm{E}-01$ & $9.4 \mathrm{E}-01$ & $2.8 \mathrm{E}-01$ & $3.6 \mathrm{E}-01$ \\
\hline
\end{tabular}

\begin{tabular}{|l|l|l|l|l|l|}
\hline Table S32 contd. Life Cycle Inventories per kg crop from farm 2 \\
\hline
\end{tabular}


\begin{tabular}{|l}
\hline Phosphate fertiliser, as P2O5 \{GLO\}| market for $\mid$ Conseq, $\mathrm{U}$ \\
\hline
\end{tabular} Potassium nitrate \{GLO\}| market for $\mid$ Conseq, $\mathrm{U}$

Transport, passenger car, small size, petrol, EURO 5 \{GLO\}| market for $\mid$ Conseq, U

\begin{tabular}{|l|l}
\hline Waste & \\
\hline Cluminium (waste treatment) (US-NPCC)/ recycling of aluminium (Conseq, $U$
\end{tabular}

Copper (waste treatment) \{US-NPCC | recycling of copper | Conseg U

Inert waste, for final disposal $\{$ US $\} \mid$ market for $\mid$ Conseq, $U$

PE (waste treatment) \{US-NPCC $\} \mid$ recycling of PE $\mid$ Conseq, $U$

PP (waste treatment) $\{$ US-NPCC $\} \mid$ recycling of PP $\mid$ Conseq, $U$

\begin{tabular}{|l|l}
\hline Steel and iron (waste treatment) \{US-NPCC $\mid$ recycling of steel and iron $\mid$ Conseg $U$ & $\mathrm{~kg}$ \\
\hline
\end{tabular}

\begin{tabular}{|l|l|l|l|l|l|}
\hline $\mathrm{kg}$ & $1.6 \mathrm{E}-03$ & $1.8 \mathrm{E}-03$ & $5.5 \mathrm{E}-04$ & $1.4 \mathrm{E}-03$ & $2.0 \mathrm{E}-03$ \\
\hline $\mathrm{kg}$ & $8.6 \mathrm{E}-04$ & $9.5 \mathrm{E}-04$ & $3.0 \mathrm{E}-04$ & $7.6 \mathrm{E}-04$ & $1.1 \mathrm{E}-03$ \\
\hline $\mathrm{m}^{3}$ & $1.9 \mathrm{E}-02$ & $2.1 \mathrm{E}-02$ & $6.6 \mathrm{E}-03$ & $1.7 \mathrm{E}-02$ & $2.4 \mathrm{E}-02$ \\
\hline $\mathrm{km}$ & $1.6 \mathrm{E}+00$ & $1.8 \mathrm{E}+00$ & $5.5 \mathrm{E}-01$ & $1.4 \mathrm{E}+00$ & $2.0 \mathrm{E}+00$ \\
\hline & & & & & \\
$\mathrm{kg}$ & $1.2 \mathrm{E}-06$ & $1.3 \mathrm{E}-06$ & $4.1 \mathrm{E}-07$ & $1.0 \mathrm{E}-06$ & $1.5 \mathrm{E}-06$ \\
\hline $\mathrm{kg}$ & $1.2 \mathrm{E}-06$ & $1.3 \mathrm{E}-06$ & $4.1 \mathrm{E}-07$ & $1.0 \mathrm{E}-06$ & $1.5 \mathrm{E}-06$ \\
\hline $\mathrm{kg}$ & $3.3 \mathrm{E}+00$ & $3.6 \mathrm{E}+00$ & $1.1 \mathrm{E}+00$ & $2.9 \mathrm{E}+00$ & $4.1 \mathrm{E}+00$ \\
$\mathrm{~kg}$ & $6.9 \mathrm{E}-02$ & $7.6 \mathrm{E}-02$ & $2.4 \mathrm{E}-02$ & $6.0 \mathrm{E}-02$ & $8.5 \mathrm{E}-02$ \\
\hline $\mathrm{kg}$ & $1.4 \mathrm{E}-02$ & $1.5 \mathrm{E}-02$ & $4.7 \mathrm{E}-03$ & $1.2 \mathrm{E}-02$ & $1.7 \mathrm{E}-02$ \\
\hline $\mathrm{kg}$ & $6.3 \mathrm{E}-01$ & $6.9 \mathrm{E}-01$ & $2.2 \mathrm{E}-01$ & $5.5 \mathrm{E}-01$ & $7.8 \mathrm{E}-01$ \\
\hline
\end{tabular}

\begin{tabular}{|c|c|c|c|c|c|c|}
\hline & Unit & Turnip & Tomato & Squash & Bell Pepper & Lettuce \\
\hline \multicolumn{7}{|l|}{ Material and Energy Inputs } \\
\hline \multicolumn{7}{|l|}{ Capital } \\
\hline Concrete, normal $\{$ US-NPCC $\} \mid$ production $\mid$ Conseq, $\mathrm{U}$ & $\mathrm{m}^{3}$ & $1.2 \mathrm{E}-05$ & $9.5 \mathrm{E}-06$ & $1.6 \mathrm{E}-05$ & $1.6 \mathrm{E}-05$ & $5.0 \mathrm{E}-05$ \\
\hline Extrusion, plastic film \{US-NPCC $\} \mid$ production $\mid$ Conseq, $U$ & $\mathrm{~kg}$ & $5.1 \mathrm{E}-03$ & $4.1 \mathrm{E}-03$ & $7.0 \mathrm{E}-03$ & $7.0 \mathrm{E}-03$ & $2.2 \mathrm{E}-02$ \\
\hline Extrusion, plastic pipes \{US-NPCC\}| market for | Conseq, U & $\mathrm{kg}$ & $5.6 \mathrm{E}-03$ & $4.6 \mathrm{E}-03$ & $7.8 \mathrm{E}-03$ & $7.8 \mathrm{E}-03$ & $2.4 \mathrm{E}-02$ \\
\hline Polyethylene, low density, granulate $\{\mathrm{GLO}\} \mid$ market for $\mid$ Conseq, U & $\mathrm{kg}$ & $3.6 \mathrm{E}-03$ & $3.0 \mathrm{E}-03$ & $5.0 \mathrm{E}-03$ & $5.1 \mathrm{E}-03$ & $1.6 \mathrm{E}-02$ \\
\hline Polypropylene, granulate $\{\mathrm{GLO}\} \mid$ market for $\mid$ Conseq, $\mathrm{U}$ & $\mathrm{kg}$ & $4.3 \mathrm{E}-03$ & $3.5 \mathrm{E}-03$ & $5.9 \mathrm{E}-03$ & $6.0 \mathrm{E}-03$ & $1.9 \mathrm{E}-02$ \\
\hline Polyvinylchloride, bulk polymerised $\{\mathrm{GLO}\} \mid$ market for $\mid$ Conseq, $\mathrm{U}$ & $\mathrm{kg}$ & $2.7 \mathrm{E}-03$ & $2.2 \mathrm{E}-03$ & $3.7 \mathrm{E}-03$ & $3.7 \mathrm{E}-03$ & $1.1 \mathrm{E}-02$ \\
\hline Sawnwood, hardwood, air dried, planed $\{$ RoW $\} \mid$ market for $\mid$ Conseq, $U$ & $\mathrm{~m}^{3}$ & $4.1 \mathrm{E}-05$ & $3.4 \mathrm{E}-05$ & $5.7 \mathrm{E}-05$ & $5.7 \mathrm{E}-05$ & $1.8 \mathrm{E}-04$ \\
\hline Steel, low-alloyed $\{\mathrm{GLO}\} \mid$ market for $\mid$ Conseq, $\mathrm{U}$ & $\mathrm{kg}$ & $7.4 \mathrm{E}-04$ & $6.1 \mathrm{E}-04$ & $1.0 \mathrm{E}-03$ & $1.0 \mathrm{E}-03$ & $3.2 \mathrm{E}-03$ \\
\hline Steel, low-alloyed, hot rolled $\{$ US-NPCC $\} \mid$ market for $\mid$ Conseq, U & $\mathrm{kg}$ & $2.1 \mathrm{E}-03$ & $1.7 \mathrm{E}-03$ & $2.9 \mathrm{E}-03$ & $3.0 \mathrm{E}-03$ & $9.2 \mathrm{E}-03$ \\
\hline Straw $\{\mathrm{GLO}\} \mid$ market for $\mid$ Conseq, $\mathrm{U}$ & $\mathrm{kg}$ & $4.1 \mathrm{E}-02$ & $3.4 \mathrm{E}-02$ & $5.7 \mathrm{E}-02$ & $5.7 \mathrm{E}-02$ & $1.8 \mathrm{E}-01$ \\
\hline Synthetic rubber $\{$ GLO $\} \mid$ market for $\mid$ Conseq, $\mathrm{U}$ & $\mathrm{kg}$ & $1.2 \mathrm{E}-04$ & $9.7 \mathrm{E}-05$ & $1.6 \mathrm{E}-04$ & $1.6 \mathrm{E}-04$ & $5.1 \mathrm{E}-04$ \\
\hline Transport, freight, lorry > 32 metric ton, EURO4 \{GLO\}| market for $\mid$ Conseq, U & $\mathrm{tkm}$ & $6.5 \mathrm{E}-02$ & $5.3 \mathrm{E}-02$ & $8.9 \mathrm{E}-02$ & $9.0 \mathrm{E}-02$ & $2.8 \mathrm{E}-01$ \\
\hline \multicolumn{7}{|l|}{ Operations } \\
\hline Ammonium nitrate, as $\mathrm{N}\{\mathrm{RER}\} \mid$ ammonium nitrate production $\mid$ Conseq, $\mathrm{U}$ & $\mathrm{kg}$ & $8.2 \mathrm{E}-04$ & $6.7 \mathrm{E}-04$ & $1.1 \mathrm{E}-03$ & $1.1 \mathrm{E}-03$ & $3.5 \mathrm{E}-03$ \\
\hline Extrusion, plastic film \{US-NPCC $\} \mid$ production $\mid$ Conseq, U & $\mathrm{kg}$ & $7.0 \mathrm{E}-04$ & $7.0 \mathrm{E}-04$ & $7.0 \mathrm{E}-04$ & $7.1 \mathrm{E}-04$ & $6.0 \mathrm{E}-04$ \\
\hline Occupation, urban, continuously built & $\mathrm{m}^{2} \mathrm{a}$ & $2.9 \mathrm{E}-01$ & $2.4 \mathrm{E}-01$ & $4.0 \mathrm{E}-01$ & $4.0 \mathrm{E}-01$ & $1.2 \mathrm{E}+00$ \\
\hline Petrol, unleaded $\{$ RoW $\} \mid$ market for $\mid$ Conseq, $U$ & $\mathrm{~kg}$ & $7.4 \mathrm{E}-04$ & $6.1 \mathrm{E}-04$ & $1.0 \mathrm{E}-03$ & $1.0 \mathrm{E}-03$ & $3.2 \mathrm{E}-03$ \\
\hline Phosphate fertiliser, as P2O5 \{GLO $\} \mid$ market for $\mid$ Conseq, U & $\mathrm{kg}$ & $3.3 \mathrm{E}-04$ & $2.7 \mathrm{E}-04$ & $4.5 \mathrm{E}-04$ & $4.6 \mathrm{E}-04$ & $1.4 \mathrm{E}-03$ \\
\hline Polyethylene, high density, granulate $\{\mathrm{GLO}\} \mid$ market for $\mid$ Conseq, U & $\mathrm{kg}$ & $7.0 \mathrm{E}-04$ & $7.0 \mathrm{E}-04$ & $7.0 \mathrm{E}-04$ & $7.1 \mathrm{E}-04$ & $6.0 \mathrm{E}-04$ \\
\hline Potassium sulfate, as $\mathrm{K} 2 \mathrm{O}\{\mathrm{GLO}\} \mid$ market for $\mid$ Conseq, $\mathrm{U}$ & $\mathrm{kg}$ & $1.0 \mathrm{E}-03$ & $8.2 \mathrm{E}-04$ & $1.4 \mathrm{E}-03$ & $1.4 \mathrm{E}-03$ & $4.4 \mathrm{E}-03$ \\
\hline Tap water $\{$ US-Boston $\} \mid$ market for $\mid$ Conseq, $\mathrm{U}$ & ton & $3.4 \mathrm{E}-01$ & $1.6 \mathrm{E}-01$ & $3.3 \mathrm{E}-01$ & $3.3 \mathrm{E}-01$ & $3.1 \mathrm{E}-01$ \\
\hline Transport, freight, lorry $>32$ metric ton, EURO4 $\{\mathrm{GLO}\} \mid$ market for $\mid$ Conseq, $\mathrm{U}$ & $\mathrm{tkm}$ & $1.2 \mathrm{E}-02$ & $9.6 \mathrm{E}-03$ & $1.6 \mathrm{E}-02$ & $1.6 \mathrm{E}-02$ & $5.1 \mathrm{E}-02$ \\
\hline $\begin{array}{l}\text { Transport, passenger car, large size, petrol, EURO } 4\{\text { GLO }\} \mid \text { market for transport, } \\
\text { passenger car, large size, petol, EURO } 4 \text { | Conseq, U }\end{array}$ & $\mathrm{km}$ & $3.7 \mathrm{E}-02$ & $3.1 \mathrm{E}-02$ & $5.1 \mathrm{E}-02$ & $5.2 \mathrm{E}-02$ & $1.6 \mathrm{E}-01$ \\
\hline $\begin{array}{l}\text { Transport, passenger car, large size, petrol, EURO } 4 \text { \{RER\}| transport, passenger car, } \\
\text { large size, petrol, EURO } 4 \text { | Conseq, U }\end{array}$ & $\mathrm{km}$ & $2.5 \mathrm{E}-02$ & $2.5 \mathrm{E}-02$ & $2.5 \mathrm{E}-02$ & $2.5 \mathrm{E}-02$ & $2.1 \mathrm{E}-02$ \\
\hline \multicolumn{7}{|l|}{ Direct Emissions } \\
\hline Carbon dioxide, fossil & $\mathrm{kg}$ & $2.5 \mathrm{E}-03$ & $2.1 \mathrm{E}-03$ & $3.5 \mathrm{E}-03$ & $3.5 \mathrm{E}-03$ & $1.1 \mathrm{E}-02$ \\
\hline \multicolumn{7}{|l|}{ Waste } \\
\hline Inert waste, for final disposal $\{$ US $\} \mid$ market for $\mid$ Conseq, $U^{*}$ & $\mathrm{~kg}$ & $-1.8 \mathrm{E}-01$ & $-1.5 \mathrm{E}-01$ & $-2.5 \mathrm{E}-01$ & $-2.5 \mathrm{E}-01$ & $-7.7 \mathrm{E}-01$ \\
\hline PE (waste treatment) \{US-NPCC $\} \mid$ recycling of PE $\mid$ Conseq, U & $\mathrm{kg}$ & $4.1 \mathrm{E}-03$ & $3.5 \mathrm{E}-03$ & $5.4 \mathrm{E}-03$ & $5.4 \mathrm{E}-03$ & $1.5 \mathrm{E}-02$ \\
\hline PVC (waste treatment) \{US-NPCC $\} \mid$ recycling of PVC $\mid$ Conseq, U & $\mathrm{kg}$ & $2.5 \mathrm{E}-03$ & $2.1 \mathrm{E}-03$ & $3.5 \mathrm{E}-03$ & $3.5 \mathrm{E}-03$ & $1.1 \mathrm{E}-02$ \\
\hline Rubber (waste treatment) \{US-NPCC $\} \mid$ recycling of rubber $\mid$ Conseq, $U$ & $\mathrm{~kg}$ & $5.9 \mathrm{E}-05$ & $4.8 \mathrm{E}-05$ & $8.2 \mathrm{E}-05$ & $8.2 \mathrm{E}-05$ & $2.6 \mathrm{E}-04$ \\
\hline Steel and iron (waste treatment) $\{$ US-NPCC $\} \mid$ recycling of steel and iron $\mid$ Conseq, $\mathrm{U}$ & $\mathrm{kg}$ & $1.8 \mathrm{E}-03$ & $1.4 \mathrm{E}-03$ & $2.4 \mathrm{E}-03$ & $2.5 \mathrm{E}-03$ & $7.6 \mathrm{E}-03$ \\
\hline Waste concrete gravel $\{$ US-NPCC $\} \mid$ treatment of, recycling $\mid$ Conseq, $U$ & $\mathrm{~kg}$ & $2.6 \mathrm{E}-02$ & $2.1 \mathrm{E}-02$ & $3.5 \mathrm{E}-02$ & $3.6 \mathrm{E}-02$ & $1.1 \mathrm{E}-01$ \\
\hline Waste wood, post-consumer $\{\mathrm{GLO}\} \mid$ market for $\mid$ Conseq, $\mathrm{U}$ & $\mathrm{kg}$ & $1.9 \mathrm{E}-02$ & $1.6 \mathrm{E}-02$ & $2.6 \mathrm{E}-02$ & $2.7 \mathrm{E}-02$ & $8.3 \mathrm{E}-02$ \\
\hline
\end{tabular}

* Negative number due to avoided waste from the use of used jute bags for ground cover

\begin{tabular}{|c|c|c|c|c|c|c|}
\hline & Unit & Kale & Cucumber & Collard Greens & Carrot & Cobbage \\
\hline \multicolumn{7}{|l|}{ Material and Energy Inputs } \\
\hline \multicolumn{7}{|l|}{ Capital } \\
\hline Concrete, normal $\{$ US-NPCC $\} \mid$ production $\mid$ Conseq, $U$ & $\mathrm{~m}^{3}$ & $8.6 \mathrm{E}-06$ & $1.2 \mathrm{E}-05$ & $9.8 \mathrm{E}-05$ & $2.5 \mathrm{E}-05$ & $8.6 \mathrm{E}-06$ \\
\hline Extrusion, plastic film \{US-NPCC $\} \mid$ production $\mid$ Conseq, $U$ & $\mathrm{~kg}$ & $3.7 \mathrm{E}-03$ & $5.3 \mathrm{E}-03$ & $4.3 \mathrm{E}-02$ & $1.1 \mathrm{E}-02$ & $3.8 \mathrm{E}-03$ \\
\hline Extrusion, plastic pipes $\{$ US-NPCC $\} \mid$ market for $\mid$ Conseq, $\mathrm{U}$ & $\mathrm{kg}$ & $4.2 \mathrm{E}-03$ & $5.9 \mathrm{E}-03$ & $4.8 \mathrm{E}-02$ & $1.2 \mathrm{E}-02$ & $4.2 \mathrm{E}-03$ \\
\hline Polyethylene, low density, granulate $\{\mathrm{GLO}\} \mid$ market for $\mid$ Conseq, $\mathrm{U}$ & $\mathrm{kg}$ & $2.7 \mathrm{E}-03$ & $3.8 \mathrm{E}-03$ & $3.1 \mathrm{E}-02$ & $7.8 \mathrm{E}-03$ & $2.7 \mathrm{E}-03$ \\
\hline Polypropylene, granulate $\{\mathrm{GLO}\} \mid$ market for $\mid$ Conseq, $\mathrm{U}$ & $\mathrm{kg}$ & $3.2 \mathrm{E}-03$ & $4.5 \mathrm{E}-03$ & $3.6 \mathrm{E}-02$ & $9.2 \mathrm{E}-03$ & $3.2 \mathrm{E}-03$ \\
\hline Polyvinylchloride, bulk polymerised $\{\mathrm{GLO}\} \mid$ market for $\mid$ Conseq, U & $\mathrm{kg}$ & $2.0 \mathrm{E}-03$ & $2.8 \mathrm{E}-03$ & $2.2 \mathrm{E}-02$ & $5.7 \mathrm{E}-03$ & $2.0 \mathrm{E}-03$ \\
\hline Sawnwood, hardwood, air dried, planed $\{$ RoW $\} \mid$ market for $\mid$ Conseq, U & $\mathrm{m}^{3}$ & $3.1 \mathrm{E}-05$ & $4.3 \mathrm{E}-05$ & $3.5 \mathrm{E}-04$ & $8.9 \mathrm{E}-05$ & $3.1 \mathrm{E}-05$ \\
\hline Steel, low-alloyed $\{\mathrm{GLO}\} \mid$ market for $\mid$ Conseq, $\mathrm{U}$ & $\mathrm{kg}$ & $5.5 \mathrm{E}-04$ & $7.8 \mathrm{E}-04$ & $6.3 \mathrm{E}-03$ & $1.6 \mathrm{E}-03$ & $5.5 \mathrm{E}-04$ \\
\hline Steel, low-alloyed, hot rolled $\{$ US-NPCC $\} \mid$ market for $\mid$ Conseq, U & $\mathrm{kg}$ & $1.6 \mathrm{E}-03$ & $2.2 \mathrm{E}-03$ & $1.8 \mathrm{E}-02$ & $4.6 \mathrm{E}-03$ & $1.6 \mathrm{E}-03$ \\
\hline Straw $\{\mathrm{GLO}\} \mid$ market for $\mid$ Conseq, $\mathrm{U}$ & $\mathrm{kg}$ & $3.0 \mathrm{E}-02$ & $4.3 \mathrm{E}-02$ & $3.5 \mathrm{E}-01$ & $8.8 \mathrm{E}-02$ & $3.1 \mathrm{E}-02$ \\
\hline Synthetic rubber $\{\mathrm{GLO}\} \mid$ market for $\mid$ Conseq, $\mathrm{U}$ & $\mathrm{kg}$ & $8.8 \mathrm{E}-05$ & $1.2 \mathrm{E}-04$ & $1.0 \mathrm{E}-03$ & $2.5 \mathrm{E}-04$ & $8.8 \mathrm{E}-05$ \\
\hline Transport, freight, lorry $>32$ metric ton, EURO4 $\{\mathrm{GLO}\} \mid$ market for $\mid$ Conseq, $\mathrm{U}$ & tkm & $4.8 \mathrm{E}-02$ & $6.8 \mathrm{E}-02$ & $5.5 \mathrm{E}-01$ & $1.4 \mathrm{E}-01$ & $4.8 \mathrm{E}-02$ \\
\hline \multicolumn{7}{|l|}{ Operations } \\
\hline Ammonium nitrate, as $\mathrm{N}\{\mathrm{RER}\} \mid$ ammonium nitrate production $\mid$ Conseq, $\mathrm{U}$ & $\mathrm{kg}$ & $6.1 \mathrm{E}-04$ & $8.6 \mathrm{E}-04$ & $6.9 \mathrm{E}-03$ & $1.8 \mathrm{E}-03$ & $6.1 \mathrm{E}-04$ \\
\hline Extrusion, plastic film \{US-NPCC $\} \mid$ production $\mid$ Conseq, $\mathrm{U}$ & $\mathrm{kg}$ & $7.0 \mathrm{E}-04$ & $7.2 \mathrm{E}-04$ & $7.0 \mathrm{E}-04$ & $7.0 \mathrm{E}-04$ & $7.0 \mathrm{E}-04$ \\
\hline Occupation, urban, continuously built & $\mathrm{m}^{2} \mathrm{a}$ & $2.1 \mathrm{E}-01$ & $3.0 \mathrm{E}-01$ & $2.4 \mathrm{E}+00$ & $6.2 \mathrm{E}-01$ & $2.1 \mathrm{E}-01$ \\
\hline Petrol, unleaded $\{$ RoW $\} \mid$ market for $\mid$ Conseq, $U$ & $\mathrm{~kg}$ & $5.5 \mathrm{E}-04$ & $7.8 \mathrm{E}-04$ & $6.3 \mathrm{E}-03$ & $1.6 \mathrm{E}-03$ & $5.5 \mathrm{E}-04$ \\
\hline Phosphate fertiliser, as P2O5 \{GLO $\} \mid$ market for $\mid$ Conseq, U & $\mathrm{kg}$ & $2.4 \mathrm{E}-04$ & $3.4 \mathrm{E}-04$ & $2.8 \mathrm{E}-03$ & $7.1 \mathrm{E}-04$ & $2.4 \mathrm{E}-04$ \\
\hline Polyethylene, high density, granulate $\{\mathrm{GLO}\} \mid$ market for $\mid$ Conseq, $\mathrm{U}$ & $\mathrm{kg}$ & $7.0 \mathrm{E}-04$ & $7.2 \mathrm{E}-04$ & $7.0 \mathrm{E}-04$ & $7.0 \mathrm{E}-04$ & $7.0 \mathrm{E}-04$ \\
\hline Potassium sulfate, as K2O \{GLO\}| market for $\mid$ Conseq, $\mathrm{U}$ & $\mathrm{kg}$ & $7.4 \mathrm{E}-04$ & $1.1 \mathrm{E}-03$ & $8.5 \mathrm{E}-03$ & $2.2 \mathrm{E}-03$ & $7.5 \mathrm{E}-04$ \\
\hline Tap water \{US-Boston\}| market for $\mid$ Conseq, $\mathrm{U}$ & ton & $5.2 \mathrm{E}-02$ & $1.3 \mathrm{E}-01$ & $6.0 \mathrm{E}-01$ & $7.2 \mathrm{E}-01$ & $5.3 \mathrm{E}-02$ \\
\hline Transport, freight, lorry >32 metric ton, EURO4 \{GLO\}| market for $\mid$ Conseq, U & $\mathrm{tkm}$ & $8.7 \mathrm{E}-03$ & $1.2 \mathrm{E}-02$ & $9.9 \mathrm{E}-02$ & $2.5 \mathrm{E}-02$ & $8.7 \mathrm{E}-03$ \\
\hline $\begin{array}{l}\text { Transport, passenger car, large size, petrol, EURO } 4\{\text { GLO }\} \mid \text { market for transport, } \\
\text { passenger car, large size, petol, EURO } 4 \mid \text { Conseq, U }\end{array}$ & $\mathrm{km}$ & $2.8 \mathrm{E}-02$ & $3.9 \mathrm{E}-02$ & $3.2 \mathrm{E}-01$ & $8.0 \mathrm{E}-02$ & $2.8 \mathrm{E}-02$ \\
\hline $\begin{array}{l}\text { Transport, passenger car, large size, petrol, EURO } 4 \text { \{RER }\} \mid \text { transport, passenger car, } \\
\text { large size, petrol, EURO } 4 \text { | Conseq, U }\end{array}$ & $\mathrm{km}$ & $2.5 \mathrm{E}-02$ & $2.6 \mathrm{E}-02$ & $2.5 \mathrm{E}-02$ & $2.5 \mathrm{E}-02$ & $2.5 \mathrm{E}-02$ \\
\hline \multicolumn{7}{|l|}{ Direct Emissions } \\
\hline Carbon dioxide, fossil & $\mathrm{kg}$ & $1.9 \mathrm{E}-03$ & $2.6 \mathrm{E}-03$ & $2.1 \mathrm{E}-02$ & $5.4 \mathrm{E}-03$ & $1.9 \mathrm{E}-03$ \\
\hline \multicolumn{7}{|l|}{ Waste } \\
\hline Inert waste, for final disposal $\{$ US $\} \mid$ market for $\mid$ Conseq, $U^{*}$ & $\mathrm{~kg}$ & $-1.3 \mathrm{E}-01$ & $-1.9 \mathrm{E}-01$ & $-1.5 \mathrm{E}+00$ & $-3.8 \mathrm{E}-01$ & $-1.3 \mathrm{E}-01$ \\
\hline $\mathrm{PE}$ (waste treatment) \{US-NPCC$\} \mid$ recycling of PE $\mid$ Conseq, $\mathrm{U}$ & $\mathrm{kg}$ & $3.2 \mathrm{E}-03$ & $4.3 \mathrm{E}-03$ & $3.0 \mathrm{E}-02$ & $8.0 \mathrm{E}-03$ & $3.2 \mathrm{E}-03$ \\
\hline $\mathrm{PVC}$ (waste treatment) \{US-NPCC $\} \mid$ recycling of PVC $\mid$ Conseq, $\mathrm{U}$ & $\mathrm{kg}$ & $1.9 \mathrm{E}-03$ & $2.6 \mathrm{E}-03$ & $2.1 \mathrm{E}-02$ & $5.4 \mathrm{E}-03$ & $1.9 \mathrm{E}-03$ \\
\hline Rubber (waste treatment) \{US-NPCC\}| recycling of rubber | Conseq, U & $\mathrm{kg}$ & $4.4 \mathrm{E}-05$ & $6.2 \mathrm{E}-05$ & $5.0 \mathrm{E}-04$ & $1.3 \mathrm{E}-04$ & $4.4 \mathrm{E}-05$ \\
\hline Steel and iron (waste treatment) $\{$ US-NPCC $\} \mid$ recycling of steel and iron $\mid$ Conseq, $U$ & $\mathrm{~kg}$ & $1.3 \mathrm{E}-03$ & $1.8 \mathrm{E}-03$ & $1.5 \mathrm{E}-02$ & $3.8 \mathrm{E}-03$ & $1.3 \mathrm{E}-03$ \\
\hline Waste concrete gravel \{US-NPCC $\} \mid$ treatment of, recycling $\mid$ Conseq, $\mathrm{U}$ & $\mathrm{kg}$ & $1.9 \mathrm{E}-02$ & $2.7 \mathrm{E}-02$ & $2.2 \mathrm{E}-01$ & $5.5 \mathrm{E}-02$ & $1.9 \mathrm{E}-02$ \\
\hline Waste wood, post-consumer $\{\mathrm{GLO}\} \mid$ market for $\mid$ Conseq, $\mathrm{U}$ & $\mathrm{kg}$ & $1.4 \mathrm{E}-02$ & $2.0 \mathrm{E}-02$ & $1.6 \mathrm{E}-01$ & $4.1 \mathrm{E}-02$ & $1.4 \mathrm{E}-02$ \\
\hline
\end{tabular}

* Negative number due to avoided waste from the use of used jute bags for ground cover

\begin{tabular}{|l} 
Table S33 contd. Life cycle inventories per kg crop from farm 3 \\
\hline
\end{tabular}

\begin{tabular}{|c|c|c|c|c|}
\hline & Unit & Beet & Green Bean & Scallion \\
\hline \multicolumn{5}{|l|}{ Capital } \\
\hline Concrete, normal $\{$ US-NPCC $\} \mid$ production $\mid$ Conseq, $\mathrm{U}$ & $\mathrm{m}^{3}$ & $1.8 \mathrm{E}-05$ & $9.5 \mathrm{E}-06$ & $5.3 \mathrm{E}-05$ \\
\hline Extrusion, plastic film $\{$ US-NPCC $\} \mid$ production $\mid$ Conseq, $U$ & $\mathrm{~kg}$ & $7.8 \mathrm{E}-03$ & $4.1 \mathrm{E}-03$ & $2.3 \mathrm{E}-02$ \\
\hline Extrusion, plastic pipes $\{$ US-NPCC $\} \mid$ market for $\mid$ Conseq, $U$ & $\mathrm{~kg}$ & $8.7 \mathrm{E}-03$ & $4.6 \mathrm{E}-03$ & $2.6 \mathrm{E}-02$ \\
\hline Polyethylene, low density, granulate $\{\mathrm{GLO}\} \mid$ market for $\mid$ Conseq, $\mathrm{U}$ & $\mathrm{kg}$ & $5.6 \mathrm{E}-03$ & $3.0 \mathrm{E}-03$ & $1.7 \mathrm{E}-02$ \\
\hline Polypropylene, granulate $\{\mathrm{GLO}\} \mid$ market for $\mid$ Conseq, $\mathrm{U}$ & $\mathrm{kg}$ & $6.6 \mathrm{E}-03$ & $3.5 \mathrm{E}-03$ & $2.0 \mathrm{E}-02$ \\
\hline Polyvinylchloride, bulk polymerised $\{\mathrm{GLO}\} \mid$ market for $\mid$ Conseq, $\mathrm{U}$ & $\mathrm{kg}$ & $4.1 \mathrm{E}-03$ & $2.2 \mathrm{E}-03$ & $1.2 \mathrm{E}-02$ \\
\hline Sawnwood, hardwood, air dried, planed $\{$ RoW $\} \mid$ market for $\mid$ Conseq, $U$ & $\mathrm{~m}^{3}$ & $6.4 \mathrm{E}-05$ & $3.4 \mathrm{E}-05$ & $1.9 \mathrm{E}-04$ \\
\hline
\end{tabular}




\begin{tabular}{|c|c|c|c|c|}
\hline $\begin{array}{l}\text { Steel, low-alloyed }\{\mathrm{GLO}\} \mid \text { market for } \mid \text { Conseq, U } \\
\end{array}$ & $\mathrm{kg}$ & $1.1 \mathrm{E}-03$ & $6.1 \mathrm{E}-04$ & $3.4 \mathrm{E}-03$ \\
\hline Steel, low-alloyed, hot rolled $\{$ US-NPCC $\} \mid$ market for $\mid$ Conseq, U & $\mathrm{kg}$ & $3.3 \mathrm{E}-03$ & $1.7 \mathrm{E}-03$ & $9.8 \mathrm{E}-03$ \\
\hline Straw $\{$ GLO $\} \mid$ market for $\mid$ Conseq, $\mathrm{U}$ & $\mathrm{kg}$ & $6.4 \mathrm{E}-02$ & $3.4 \mathrm{E}-02$ & $1.9 \mathrm{E}-01$ \\
\hline Synthetic rubber $\{\mathrm{GLO}\} \mid$ market for $\mid$ Conseq, $\mathrm{U}$ & $\mathrm{kg}$ & $1.8 \mathrm{E}-04$ & $9.7 \mathrm{E}-05$ & $5.4 \mathrm{E}-04$ \\
\hline Transport, freight, lorry > 32 metric ton, EURO4 \{GLO\}| market for $\mid$ Conseq, U & $\mathrm{tkm}$ & $1.0 \mathrm{E}-01$ & $5.3 \mathrm{E}-02$ & $3.0 \mathrm{E}-01$ \\
\hline \multicolumn{5}{|l|}{ Operations } \\
\hline Ammonium nitrate, as $\mathrm{N}\{\mathrm{RER}\} \mid$ ammonium nitrate production $\mid$ Conseq, $\mathrm{U}$ & $\mathrm{kg}$ & $1.3 \mathrm{E}-03$ & $6.7 \mathrm{E}-04$ & $3.8 \mathrm{E}-03$ \\
\hline Extrusion, plastic film \{US-NPCC $\} \mid$ production $\mid$ Conseq, $\mathrm{U}$ & $\mathrm{kg}$ & $7.0 \mathrm{E}-04$ & $7.0 \mathrm{E}-04$ & $6.8 \mathrm{E}-04$ \\
\hline Occupation, urban, continuously built & $\mathrm{m}^{2} \mathrm{a}$ & $4.5 \mathrm{E}-01$ & $2.4 \mathrm{E}-01$ & $1.3 \mathrm{E}+00$ \\
\hline Petrol, unleaded $\{$ RoW $\} \mid$ market for $\mid$ Conseq, $U$ & $\mathrm{~kg}$ & $1.2 \mathrm{E}-03$ & $6.1 \mathrm{E}-04$ & $3.4 \mathrm{E}-03$ \\
\hline Phosphate fertiliser, as P2O5 \{GLO\}| market for $\mid$ Conseq, $\mathrm{U}$ & $\mathrm{kg}$ & $5.1 \mathrm{E}-04$ & $2.7 \mathrm{E}-04$ & $1.5 \mathrm{E}-03$ \\
\hline Polyethylene, high density, granulate $\{\mathrm{GLO}\} \mid$ market for $\mid$ Conseq, $\mathrm{U}$ & $\mathrm{kg}$ & $7.0 \mathrm{E}-04$ & $7.0 \mathrm{E}-04$ & $6.8 \mathrm{E}-04$ \\
\hline Potassium sulfate, as $\mathrm{K} 2 \mathrm{O}\{\mathrm{GLO}\} \mid$ market for $\mid$ Conseq, $\mathrm{U}$ & $\mathrm{kg}$ & $1.6 \mathrm{E}-03$ & $8.2 \mathrm{E}-04$ & $4.6 \mathrm{E}-03$ \\
\hline Tap water \{US-Boston\}| market for $\mid$ Conseq, U & ton & $5.2 \mathrm{E}-01$ & $2.7 \mathrm{E}-02$ & $4.5 \mathrm{E}-02$ \\
\hline Transport, freight, lorry $>32$ metric ton, EURO4 $\{\mathrm{GLO}\} \mid$ market for $\mid$ Conseq, $\mathrm{U}$ & $\mathrm{tkm}$ & $1.8 \mathrm{E}-02$ & $9.6 \mathrm{E}-03$ & $5.4 \mathrm{E}-02$ \\
\hline $\begin{array}{l}\text { Transport, passenger car, large size, petrol, EURO } 4\{\text { GLO }\} \mid \text { market for transport, } \\
\text { passenger car, large size, petol, EURO } 4 \mid \text { Conseq, U }\end{array}$ & $\mathrm{km}$ & $5.8 \mathrm{E}-02$ & $3.1 \mathrm{E}-02$ & $1.7 \mathrm{E}-01$ \\
\hline $\begin{array}{l}\text { Transport, passenger car, large size, petrol, EURO } 4 \text { \{RER }\} \mid \text { transport, passenger car, } \\
\text { large size, petrol, EURO } 4 \text { | Conseq, U }\end{array}$ & $\mathrm{km}$ & $2.5 \mathrm{E}-02$ & $2.5 \mathrm{E}-02$ & 2.4E-02 \\
\hline \multicolumn{5}{|l|}{ Direct Emissions } \\
\hline Carbon dioxide, fossil & $\mathrm{kg}$ & $3.9 \mathrm{E}-03$ & $2.1 \mathrm{E}-03$ & $1.2 \mathrm{E}-02$ \\
\hline \multicolumn{5}{|l|}{ Waste } \\
\hline Inert waste, for final disposal $\{$ US $\} \mid$ market for $\mid$ Conseq, $U^{*}$ & $\mathrm{~kg}$ & $-2.8 \mathrm{E}-01$ & $-1.5 \mathrm{E}-01$ & $-8.2 \mathrm{E}-01$ \\
\hline $\mathrm{PE}$ (waste treatment) $\{$ US-NPCC $\} \mid$ recycling of PE $\mid$ Conseq, $\mathrm{U}$ & $\mathrm{kg}$ & $6.0 \mathrm{E}-03$ & $3.5 \mathrm{E}-03$ & $1.6 \mathrm{E}-02$ \\
\hline PVC (waste treatment) \{US-NPCC $\} \mid$ recycling of PVC $\mid$ Conseq, $\mathrm{U}$ & $\mathrm{kg}$ & $3.9 \mathrm{E}-03$ & $2.1 \mathrm{E}-03$ & $1.2 \mathrm{E}-02$ \\
\hline Rubber (waste treatment) $\{$ US-NPCC $\} \mid$ recycling of rubber $\mid$ Conseq, $U$ & $\mathrm{~kg}$ & $9.2 \mathrm{E}-05$ & $4.8 \mathrm{E}-05$ & $2.7 \mathrm{E}-04$ \\
\hline Steel and iron (waste treatment) $\{$ US-NPCC $\} \mid$ recycling of steel and iron $\mid$ Conseq, $\mathrm{U}$ & $\mathrm{kg}$ & $2.7 \mathrm{E}-03$ & $1.4 \mathrm{E}-03$ & $8.1 \mathrm{E}-03$ \\
\hline Waste concrete gravel \{US-NPCC $\} \mid$ treatment of, recycling $\mid$ Conseq, $\mathrm{U}$ & $\mathrm{kg}$ & $4.0 \mathrm{E}-02$ & $2.1 \mathrm{E}-02$ & $1.2 \mathrm{E}-01$ \\
\hline Waste wood, post-consumer $\{\mathrm{GLO}\} \mid$ market for $\mid$ Conseq, $\mathrm{U}$ & $\mathrm{kg}$ & $3.0 \mathrm{E}-02$ & $1.6 \mathrm{E}-02$ & $8.8 \mathrm{E}-02$ \\
\hline
\end{tabular}

Two metrics are assessed in this LCA: GWP and land use. GWP is assessed using the IPCC 2013 methodology over a 100 year time horizon ${ }^{12}$. Land use is assessed using the ReCiPe LCIA methodology ${ }^{13}$, which is an un-weighted method for accounting land use (it is time weighted in that it measure area $\times$ time, but since the time component is equal to a single year for all UF operations here and the MRIO model, the time weighting is inconsequential here). ReCiPe does differentiate between urban and agricultural land occupation. Here we sum both land uses to account for total land use by UF, both indirect and direct. Table S34 outlines the impacts for each product from the UF operations for both GWP and land use.

\begin{tabular}{|c|c|c|c|}
\hline Crop & Farm & GWP $\left(\mathrm{kg} \mathrm{CO}^{2} \mathrm{e} / \mathrm{kg}\right.$ crop$)$ & Land use $\left(\mathrm{m}^{2} / \mathbf{k g}\right)$ \\
\hline Beet & 3 & 0.399 & 0.713 \\
\hline Bell Pepper & 1 & 0.156 & 1.542 \\
\hline Bell Pepper & 2 & 1.165 & 0.245 \\
\hline Bell Pepper & 3 & 0.304 & 0.638 \\
\hline Cabbage & 3 & 0.116 & 0.342 \\
\hline Carrot & 2 & 1.793 & 0.377 \\
\hline Carrot & 3 & 0.549 & 0.989 \\
\hline Collard Greens & 3 & 1.218 & 3.851 \\
\hline Cucumber & 2 & 0.706 & 0.149 \\
\hline Cucumber & 3 & 0.181 & 0.479 \\
\hline Eggplant & 1 & 0.127 & 1.417 \\
\hline Green Beans & 2 & 2.547 & 0.536 \\
\hline Green Beans & 3 & 0.114 & 0.374 \\
\hline Kale & 2 & 2.256 & 0.475 \\
\hline Kale & 3 & 0.153 & 0.518 \\
\hline Lettuce & 1 & 0.263 & 5.244 \\
\hline Lettuce & 2 & 2.088 & 0.437 \\
\hline Lettuce & 3 & 0.448 & 1.798 \\
\hline Radish & 2 & 0.915 & 0.193 \\
\hline Scallion & 2 & 3.062 & 0.644 \\
\hline Scallion & 3 & 0.551 & 2.092 \\
\hline Squash & 3 & 0.302 & 0.633 \\
\hline Tomato & 1 & 0.104 & 0.880 \\
\hline Tomato & 2 & 0.625 & 0.129 \\
\hline Tomato & 3 & 0.134 & 0.344 \\
\hline Turnip & 2 & 1.547 & 0.326 \\
\hline Turnip & 3 & 0.261 & 0.462 \\
\hline
\end{tabular}

\section{Comparative performance of UF and conventional agriculture}

GWP for the conventional food are taken from Heller and Keoleian's work on the GWP impacts of the US diet ${ }^{14}$. Their work includes a review of LCAs of different food products, including the range of reported findings and average across studies. Here we use their reported averages as a proxy for conventional agriculture. As their numbers are only for production, we add on transport impacts in accordance with Pirog and Benjamin's work on 'food miles' for conventional food products heading to Iowa (data for the US northeast remain in absentia) ${ }^{15}$. Transport impacts are taken as $9.7 * 10^{-5} \mathrm{~kg}$ $\mathrm{CO}_{2} \mathrm{e} / \mathrm{kgkm}$ (ecoinvent 3.2 process 'Transport, freight, lorry $>32$ metric ton, EURO5 \{RER \}| transport, freight, lorry >32 metric ton, EURO5 |Conseq, U'). Land use is taken as direct land occupation: calculated as the total 3 year average (2012-2014) annual US production divided by the total US cultivated area from the USDA annual vegetable statistics (beet, eggplant, kale, collards, turnip, scallion taken from 2002 survey) ${ }^{16,17}$. Direct land use is taken here as this is far and away the largest driver of this indicator for vegetal foods and should cover nearly $100 \%$ of land use. Values are corrected for food losses from the USDA LAFA statistics ${ }^{2}$. Table S35 outlines the impacts of the conventional goods for both GWP and land use.

\begin{tabular}{|c|c|c|c|c|c|c|}
\hline Product & $\begin{array}{l}\text { Transport } \\
(\mathrm{km})\end{array}$ & Losses (\%) & $\begin{array}{l}\text { GWP - production } \\
\left(\mathrm{kg} \mathrm{CO}_{2} \mathrm{e} / \mathrm{kg}\right)\end{array}$ & $\begin{array}{l}\mathrm{GWP}-\text { transport } \\
\left(\mathrm{kg} \mathrm{CO} \mathrm{O}_{2} \mathrm{e} / \mathrm{kg}\right)\end{array}$ & $\begin{array}{l}\text { GWP - total (kg } \\
\left.\mathrm{CO}_{2} \mathrm{e} / \mathrm{kg}\right)\end{array}$ & Land Use ( $\left.\mathbf{m}^{2} / \mathbf{k g}\right)$ \\
\hline Beet & 1759 & 6.5 & 0.33 & 0.28 & 0.65 & 0.40 \\
\hline Bell Pepper & 1589 & 7.8 & 0.88 & 0.25 & 1.23 & 0.29 \\
\hline Cabbage & 719 & 6.5 & 0.12 & 0.11 & 0.25 & 0.27 \\
\hline Carrot & 1838 & 5.1 & 0.53 & 0.29 & 0.86 & 0.28 \\
\hline Collard Greens & 1815 & 37.5 & 0.33 & 0.29 & 0.99 & 1.10 \\
\hline Cucumber & 1277 & 6.1 & 0.66 & 0.20 & 0.92 & 0.48 \\
\hline Eggplant & 1277 & 21.3 & 1.30 & 0.20 & 1.91 & 0.43 \\
\hline Green Beans & 1313 & 18.4 & 0.73 & 0.21 & 1.15 & 1.93 \\
\hline Kale & 1815 & 39.2 & 0.33 & 0.29 & 1.01 & 0.75 \\
\hline Lettuce & 1823 & 7.7 & 1.08 & 0.29 & 1.48 & 0.27 \\
\hline Radish & 1759 & 21 & 0.33 & 0.28 & 0.77 & 1.25 \\
\hline Scallion & 1759 & 9.8 & 0.33 & 0.28 & 0.67 & 0.18 \\
\hline Squash & 1277 & 12.5 & 0.09 & 0.20 & 0.33 & 0.64 \\
\hline Tomato & 1569 & 5.2 & 0.67 & 0.25 & 0.97 & 0.34 \\
\hline Turnip & 1815 & 6.5 & 0.33 & 0.29 & 0.66 & 0.80 \\
\hline
\end{tabular}


Combining primary data on yields from the UF operations, we calculate the marginal change in environmental performance of Boston per meter UF cultivating vegetable $x, \frac{d I_{x}}{d A}$, as:

(4) $\quad \frac{d I_{x}}{d A}=\frac{d m_{i}}{d A}\left(i_{x, U A}-i_{x, \text { conv }}\right)$

Where $\frac{d m_{i}}{d A}$ is the change in mass of vegetable per unit area in kilograms (annual yield), $i_{x, U A}$, the environmental impact from producing one kilogram of vegetable $x$ with UF, and $i_{x, \text { conv }}$ the environmental impact of producing one kilogram of vegetable $x$ with conventional agriculture (crediting for the substituted conventional crop). Table S36 outlines the predicted change in Boston's food-borne environmental impacts by implementing UF. It should be noted that the yield for UF includes 'dead space' on the farm where cultivation is not occurring (e.g. sheds, footpaths, etc.) and not just productive area. Where farms 1 and 3 produce the same product, the average yield and environmental burdens have been used.

\begin{tabular}{|c|c|c|c|c|}
\hline Crop & Farm(s) & Yield $\left(\mathbf{k g} / \mathbf{m}^{2}\right)$ & $\begin{array}{l}\text { Marginal GWP Shift }\left(\mathrm{kg} \mathrm{CO}_{2} \mathrm{e} / \mathrm{m}^{2}\right. \\
\text { UF) }\end{array}$ & Marginal Land Use Shift $\left(\mathrm{m}^{2} / \mathrm{m}^{2} \mathrm{UF}\right)$ \\
\hline Beet & 3 & 2.26 & -0.57 & 0.70 \\
\hline Bell Pepper & 1 and 3 & 2.30 & -2.29 & 1.84 \\
\hline Bell Pepper & 2 & 2.44 & -0.15 & -0.65 \\
\hline Cabbage & 3 & 4.70 & 0.43 & 0.36 \\
\hline Carrot & 2 & 1.59 & 1.47 & 0.16 \\
\hline Carrot & 3 & 1.63 & -0.51 & 1.16 \\
\hline Collard Greens & 3 & 0.41 & 0.10 & 1.14 \\
\hline Cucumber & 2 & 5.28 & -1.11 & -1.73 \\
\hline Cucumber & 3 & 3.34 & -2.46 & -0.01 \\
\hline Eggplant & 1 & 2.27 & -4.04 & 2.24 \\
\hline Green Beans & 3 & 4.27 & -3.06 & -4.36 \\
\hline Green Beans & 2 & 1.12 & 1.57 & -1.56 \\
\hline Kale & 2 & 1.26 & 1.57 & -0.35 \\
\hline Kale & 3 & 4.72 & -4.24 & -1.96 \\
\hline Lettuce & 1 and 3 & 0.80 & -0.90 & 2.61 \\
\hline Lettuce & 2 & 0.80 & 0.49 & 0.14 \\
\hline Radish & 2 & 3.11 & 0.45 & -3.29 \\
\hline Scallion & 2 & 0.93 & 2.22 & 0.43 \\
\hline Scallion & 3 & 0.76 & -0.25 & 1.42 \\
\hline Squash & 3 & 2.54 & -0.08 & -0.01 \\
\hline Tomato & 1 and 3 & 2.94 & -2.50 & 0.80 \\
\hline Tomato & 2 & 4.70 & -1.61 & -0.99 \\
\hline Turnip & 2 & 1.84 & 1.63 & -0.86 \\
\hline Turnip & 3 & 3.50 & -1.39 & -1.17 \\
\hline
\end{tabular}

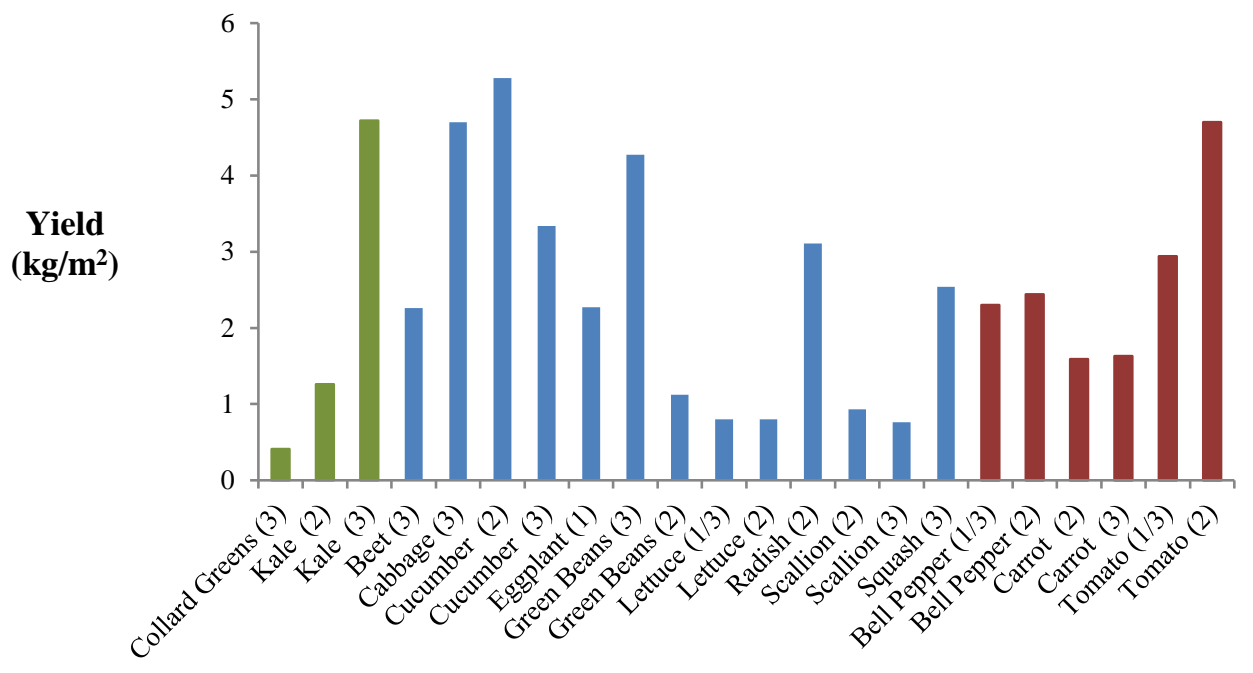

Figure 4. Yield for dark green (green), other (blue) and red and orange (red) vegetables. Farm(s) listed in brackets.

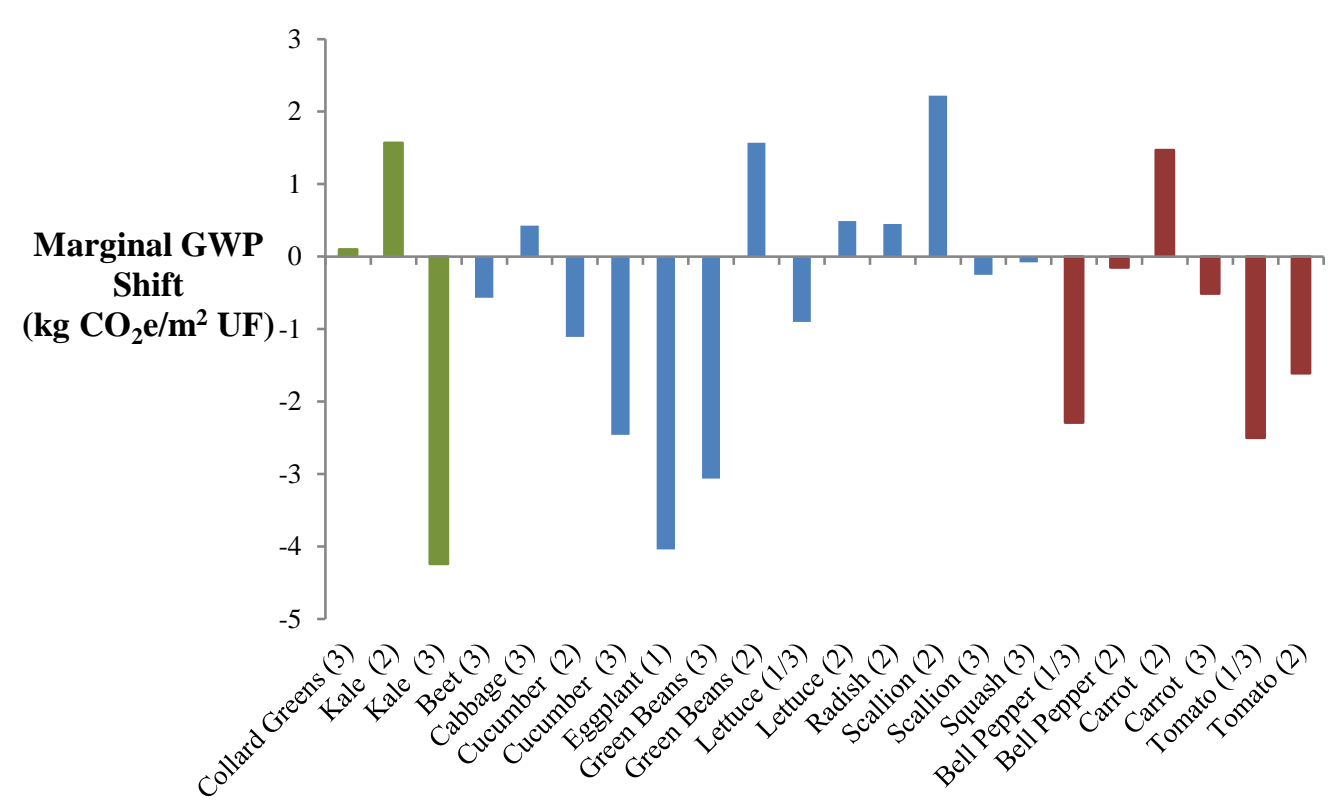

Figure 5. Marginal GWP shift per square meter UF grown for dark green (green), other (blue) and red and orange (red) vegetables. Farm(s) listed in bracets. 


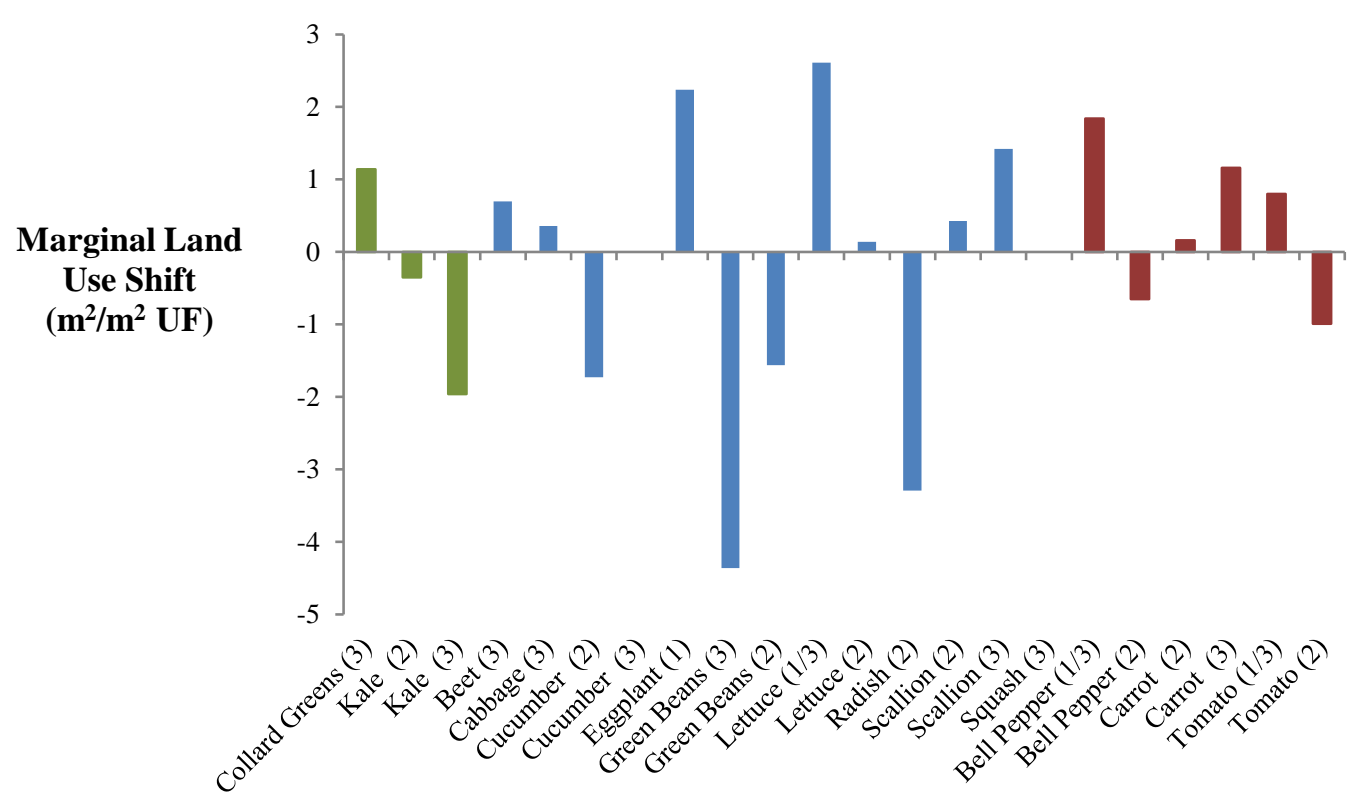

Figure 6. Marginal land use shift per square meter UF grown for dark green (green), other (blue) and red and orange (red) vegetables. Farm(s) listed in bracets.

\section{UF Space Availability}

Space for UF in Boston was estimated for ground and roof. Potential ground space was estimated by two methods: subtractive and additive. Roof space is performed in an additive manner. In this assessment, soil contamination is not considered when assessing the suitability of a piece of land for UF. Soil contamination is a major issue in US cities, particularly older cities with industrial heritage ${ }^{18}$. Moreover, shading effects from buildings are also ignored. As such, these estimates should be viewed as upper bounds for UF available space in Boston for both ground methods.

Ground Space - Additive

The additive approach for UF space starts with the assumption that the area of UF space in Boston is $0 \mathrm{~m}^{2}$. Then utilizing a variety of data sources, we look at individual pieces of land, assesse their suitability for UF and add them to amount of space suitable for UF. The data sources are the 2016 Tax Assessment Parcel and open space maps, sourced from the City of Boston's Open Data Initiative ${ }^{19}$ and the Massachusetts land use map from their geographic information system (GIS) data repository ${ }^{20}$.

Tax assessment parcels data for the year 2016 includes all tax assessment parcels in Boston $(166,248)$ including their land use according to the Massachusetts property classification system under the 'PTYPE' field in the raw data. Table S37 outlines the land uses we consider suitable for UF as they are not currently occupied by buildings or other productive land uses.

\begin{tabular}{|l|l|}
\hline \multicolumn{2}{|l|}{ Table S37. UF suitable property types from tax assessment data } \\
\hline Land Use Code ('PTYPE') & Description \\
\hline 130 & Residential land \\
\hline 131 & Residential land (secondary) \\
\hline 132 & Residential land (unusable) \\
\hline 390 & Commercial land \\
\hline 391 & Commercial land (secondary) \\
\hline 392 & Commercial land (unusable) \\
\hline 440 & Industrial land \\
\hline 441 & Industrial land (secondary) \\
\hline 442 & Industrial land (unusable) \\
\hline 337 & Parking lot \\
\hline 359 & Condo parking (commercial) \\
\hline 387 & Pay parking lot \\
\hline 108 & Condo parking (residential) \\
\hline 119 & Residential parking lot \\
\hline
\end{tabular}

Parking lots have been included here to test the impact of their inclusion on the results, since they could be considered transitional land uses. Moreover, some of the parking lots are subterranean, making them unsuitable for the UF forms considered here, though this is not indicated by the parcel assessment data. Results include assessments with and without parking included to gauge the sensitivity of the results to their inclusion.

Community garden data includes the locations of existing UF in the city as designated by the Open Space map in the city's data repository. We assume that all operating community gardens are valid for this assessment.

Lastly, the state land use map from 2005 is used to include the land uses outlined in table S38 as described by the field 'LUCODE' in the data.

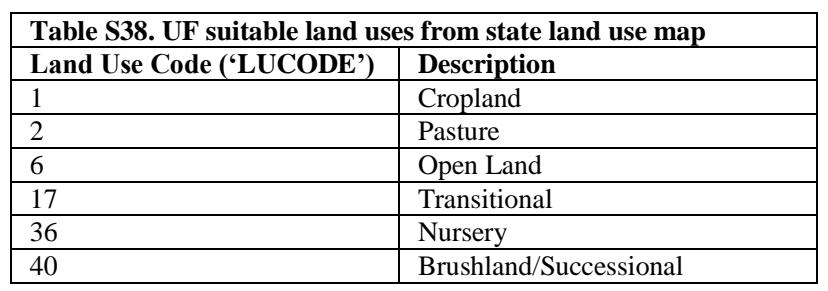

Data are imported into the GIS software QGIS 2.4.0 and corrected for two issues:

- Residential and condo parking lots are checked for double counting, since the same assessment parcel are listed multiple times if the different parking spots on the same piece of area are owned by different individuals

- Where UF suitable plots intersected, the overlapping portion is subtracted from one of the layers. See figure 7.

- Plots with average slopes greater than $10^{\circ}$, as determined from digital elevation models provided by the National Oceanic and Atmospheric Administration (NOAA) $)^{21}$, were deemed too steep for agriculture and excluded. 


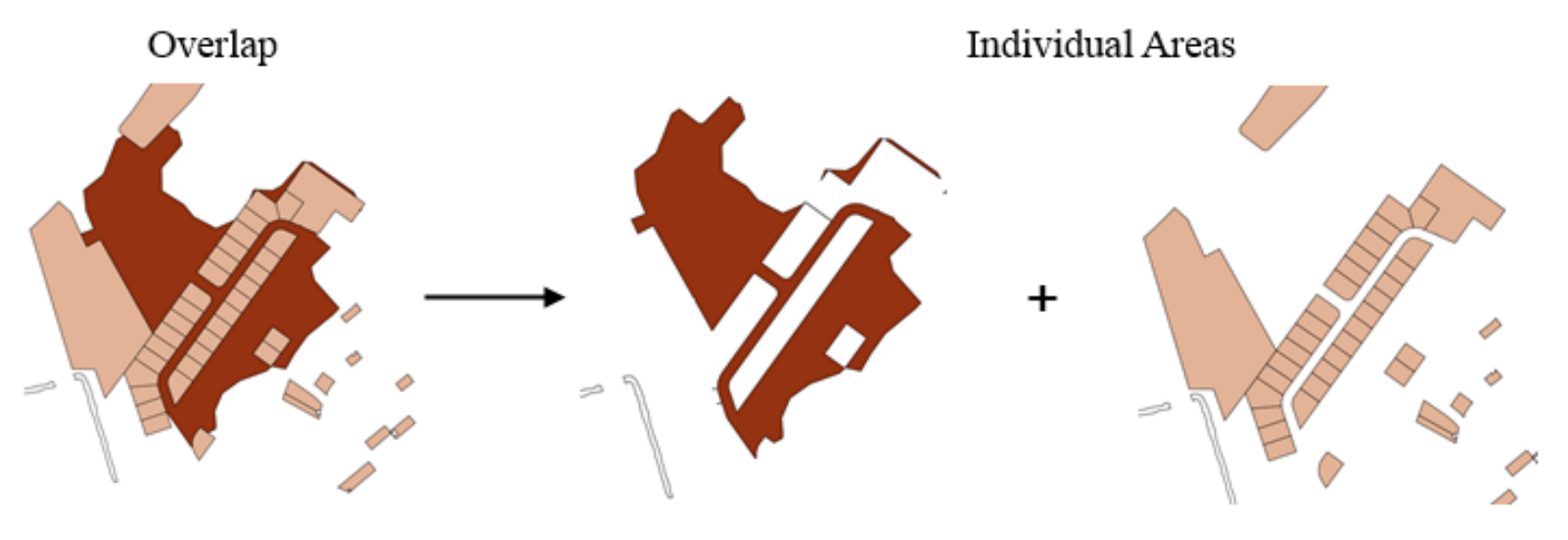

Figure 7. The disaggregation of overlapping areas in QGIS.

With a unique set of non-intersecting UF suitable plots, the area of each plot is calculated in QGIS and added to estimate total UF available space for Boston. Results are listed in table S39. QGIS also allows one to determine if an UF suitable plot lies within a block-group, providing area totals for each block-group (not shown here, but shown in Figures 2 a-b of the article).

\begin{tabular}{|c|c|c|c|c|}
\hline Land Use & $\begin{array}{l}\text { Number of UF } \\
\text { Suitable Sites }\end{array}$ & $\begin{array}{l}\text { Average Site Size (standard deviation) } \\
\mathbf{m}^{2}\end{array}$ & Total UF Space $\left(\mathbf{m}^{2}\right)$ & \begin{tabular}{|l} 
Total UF Space (acres) \\
\end{tabular} \\
\hline Vacant residential* & 5865 & $412(1637)$ & 2421080 & 599 \\
\hline Vacant commercial* & 1267 & $1026(3826)$ & 1300847 & 322 \\
\hline Vacant industrial* & 162 & $1375(3668)$ & 222860 & 55 \\
\hline Existing community gardens & 123 & $1122(2235)$ & 138063 & 34 \\
\hline Pasture & 1 & $13184(0)$ & 13184 & 3 \\
\hline Transitional & 34 & $10271(13569)$ & 349228 & 86 \\
\hline Nursery & 15 & $10127(11699)$ & 151906 & 38 \\
\hline Cropland & 14 & $6395(7739)$ & 89525 & 22 \\
\hline Brushland/Successional & 17 & $24979(53946)$ & 421542 & 104 \\
\hline Open Land & 159 & $13825(29049)$ & 2198200 & 544 \\
\hline Residential Parking* & 262 & $805(4080)$ & 210850 & 52 \\
\hline Commercial Parking* & 630 & $892(2698)$ & 562124 & 139 \\
\hline Boston Total & 8549 & $944(5740)$ & 8079409 & 2000 \\
\hline
\end{tabular}

Ground Space - Subtractive

Contrasting with the additive method, here we start with the assumption that $100 \%$ of Boston is suitable for UF and then subtract those areas deemed unsuitable for farming. As with the additive approach, overlapping areas are removed to avoid double counting. Table S40 lists the land types considered unsuitable for ground-based US, their areas and the total UF available land in Boston using the subtractive estimation method.

\begin{tabular}{|c|c|c|c|}
\hline Land Type & Data Source & Total Area (m2) & Total Area (acres) \\
\hline Steep areas & $\mathrm{NOAA}^{21}$ & 480475 & 119 \\
\hline Parks and sports fields & MassGIS ('OpenSpace' dataset)22 & 2818969 & 698 \\
\hline Protected open space & MassGIS ('OpenSpace' dataset) ${ }^{22}$ & 19461607 & 4817 \\
\hline Temporarily protected open space & MassGIS ('OpenSpace' dataset) ${ }^{22}$ & 11443 & 3 \\
\hline Cemeteries & MassGIS ('OpenSpace' dataset) ${ }^{22}$ & 3186303 & 789 \\
\hline Buildings & $\begin{array}{l}\text { Boston Open Data('Buildings' } \\
\text { dataset) }{ }^{19}\end{array}$ & 21946457 & 5433 \\
\hline $\begin{array}{l}\text { Impervious surfaces (roads, } \\
\text { sidewalks, etc.) - buildings } \\
\text { removed }\end{array}$ & $\begin{array}{l}\text { Boston Open Data('Impervious } \\
\text { Surfaces' dataset) }{ }^{19}\end{array}$ & 35926938 & 8893 \\
\hline Airport & Boston Open Data ${ }^{19}$ & 6172008 & 1528 \\
\hline Total & - & 90004200 & 22278 \\
\hline \multicolumn{4}{|l|}{ Total Boston Area } \\
\hline Boston Total Area & $\begin{array}{l}\text { Boston Open Data ('Boundary' } \\
\text { dataset) }\end{array}$ & 125095606 & 30964 \\
\hline \multirow{2}{*}{\multicolumn{4}{|c|}{ Total UF Area }} \\
\hline & & & 8846 \\
\hline
\end{tabular}

As with the additive scenario, QGIS is used to allocate available space to the block-groups in Boston.

Rooftop Space

The first step in estimating the amount of rooftop area available for UF in Boston is to get a clean data set of pertinent information of the Boston building stock. Davila and colleagues already outlined the process in detail ${ }^{23}$, but in a nutshell it involves combining three datasets: the Boston property tax assessment for the year $2014^{24}$, the 2016 tax parcel assessment data ${ }^{19}$ and the geospatial building data for Boston ${ }^{19}$.

The property tax assessment is required as it is the most up to date and detailed assessment of building attributes for the city and contains all buildings and sub-units within buildings. Because of the latter point, it contains double counting of buildings that contain multiple apartment units. Double counted units were removed using a Python 2.7 script which identifies buildings with multiple units based on the 'CM ID' field. While consolidating multiple units to a single entry, we also assign the heating and cooling type of the building based on the majority heat and cooling types for the units within the building. This initial data parsing reduces the tax records from 164,092 entries to 100,858 entries.

Although the tax records data contains the most detailed information, they contain no spatial data and cannot be mapped nor attributed to block-groups. To overcome this we link the 10 digit property ID key 'Parcel_ID' in the tax record with the synonymous 'PID_LONG' key in the spatially explicit tax assessment data. Minor mismatches between the datasets shave the number of entries down to 98,865. Table S41 outlines the various fields utilized in this process and their purposes.

\begin{tabular}{|l|l|l|}
\hline \multicolumn{2}{|l|}{ Table S41. Fields used in joining tax data sets } \\
\hline Field & Dataset & Purpose \\
\hline CM_ID & 2014 Tax records & Identify duplicate building entries \\
\hline U_Heat & 2014 Tax records & Identify the predominant heating type in multi-unit dwellings \\
\hline U_AC & 2014 Tax records & Identify if a majority of units have air conditioning in multi-unit dwellings \\
\hline Parcel_ID & 2014 Tax records & Join data tax record data with tax parcel assessment polygons \\
\hline PID_Long & Tax parcel assessment data & Join tax parcel assessment polygons with 2014 tax record data \\
\hline
\end{tabular}


Although this dataset is spatially explicit, it still contains numerous errors in terms of non-existent buildings, improper building footprints and building types that are not suitable for UF applications. Moreover, the tax data does not contain reliable estimates of building heights. To overcome this we join the generated parcel data set with the building data set, since the latter contains information on building types and can be used to more accurately calculate building footprints. The spatially explicit data is then mapped as polygons in QGIS, converted to centroids and spatially joined to building polygon data. Figure 5 illustrates this process in QGIS.

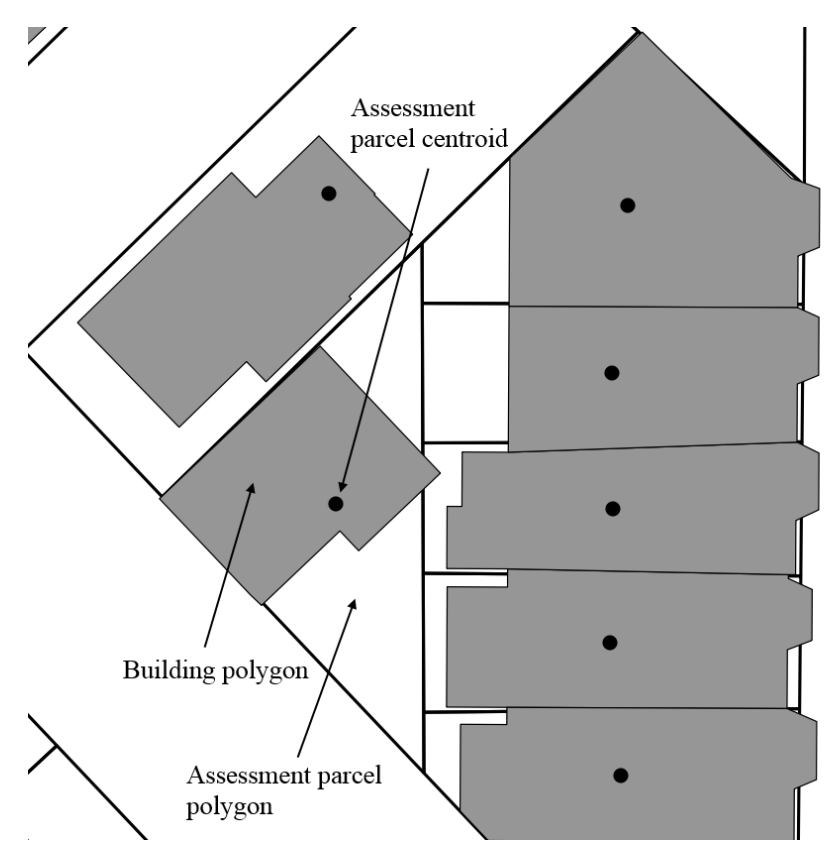

Figure 8. Joining the tax data with the building polygons

Building data is also spatially joined with data on historic preservation districts from the Boston Open Data Initiative ${ }^{19}$, as buildings in these districts are not permitted to make changes to their exterior appearance, and should be excluded from UF use. The four joined datasets contain the necessary information for estimating UF building space in Boston, outlined in table S42.

\begin{tabular}{|l|l|l|}
\hline \multicolumn{2}{|l|}{ Table S42. Properties used for calculating UF suitability } & Dataset \\
\hline Building Property & Key & 2014 Tax assessment data \\
\hline Year of construction & 'YR_BUILT' & 2014 Tax assessment data \\
\hline Number of floors & 'NUM_FLOORS' & 2014 Tax assessment data \\
\hline Roof type & 'R_ROOF_TYY' & 2014 Tax assessment data \\
\hline Heating type & 'R_HEAT_TYP' & 2014 Tax assessment data \\
\hline Presence of air conditioning & 'R_AC' & Building data \\
\hline Ground elevation & 'GROUND_ELE' & Building data \\
\hline Roof elevation & 'ROOF_LLE' & Building data \\
\hline Building Type & 'IEL_TYPE' & Building data \\
\hline Building Area & Calculated in QGIS & Historic Districts \\
\hline Presence in historic preservation district & Generated in QGIS with spatial join & \\
\hline
\end{tabular}

The final step in cleaning the building data is to remove buildings lacking information on year built, height (either no data on number of floors or incomplete elevation data) and unsuitable for UF. The latter is done using the 'IEL_TYPE' key of the building data by excluding ruins, foundations, etc. After all of the manipulations, the cleaned dataset of collated building and tax data contains 76,170 buildings $(69,857$ when historical buildings are excluded).

Actually determining the area of Boston's buildings available for UF is impossible since we lack structural analyses of the buildings that would allow us to determining their individual capacities for supporting the load of a rooftop farm. However, we can use three indicators to estimate UF rooftop space: building age, building height and roof type.

Building age is justified in the sense that the introduction of building codes and standards has led to the gradual infiltration of more structurally sound buildings through mandated snow loading capacity, etc. Thus, here we assume that older buildings are less likely to be suited for UF than new ones. This is a gross simplification, since old buildings, particularly older factories and cast iron buildings are designed to support significantly heavier loads than they are burdened with not in their post-industrial uses. As such, we run multiple scenarios building age is used as a cutoff for UF consideration. The cutoff construction years range from 1900 to 2000 in ten year intervals. This covers around 3/4 of Boston's building stock by both number of buildings and area. Figure 6 is a histogram showing the effects of different construction year cutoffs on the number and area of buildings considered.

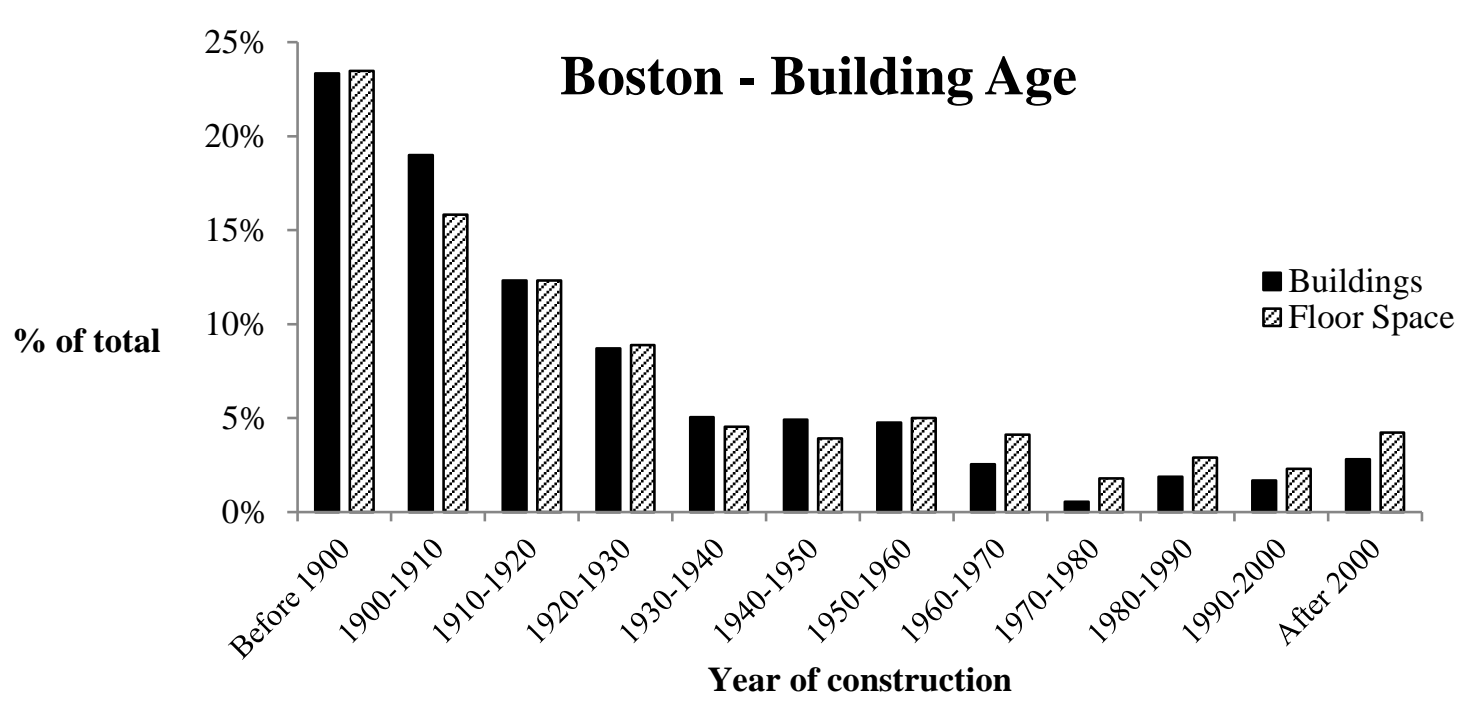

Figure 9. Histogram of building numbers and area within different intervals of construction years. 
Height is also a natural limiting factor on UF suitability, since stronger winds above certain heights not only pose a challenge to the stability of the growing medium, but also a safety issue to farm workers. Heights are taken as the difference of ground and roof elevations in the building data, and where these are lacking, the number of floors times the average floor height of $3.42 \mathrm{~m}$ as determined from those buildings within the building data set that contain both number of floors and elevation data. Looking at the histogram of number of buildings and building area with within different height ranges in Figure 7, it can be seen that only small fraction of Boston's building stock is over $30 \mathrm{~m}$ tall, and hence this is taken as the maximum allowable height for a building to be considered UF appropriate.

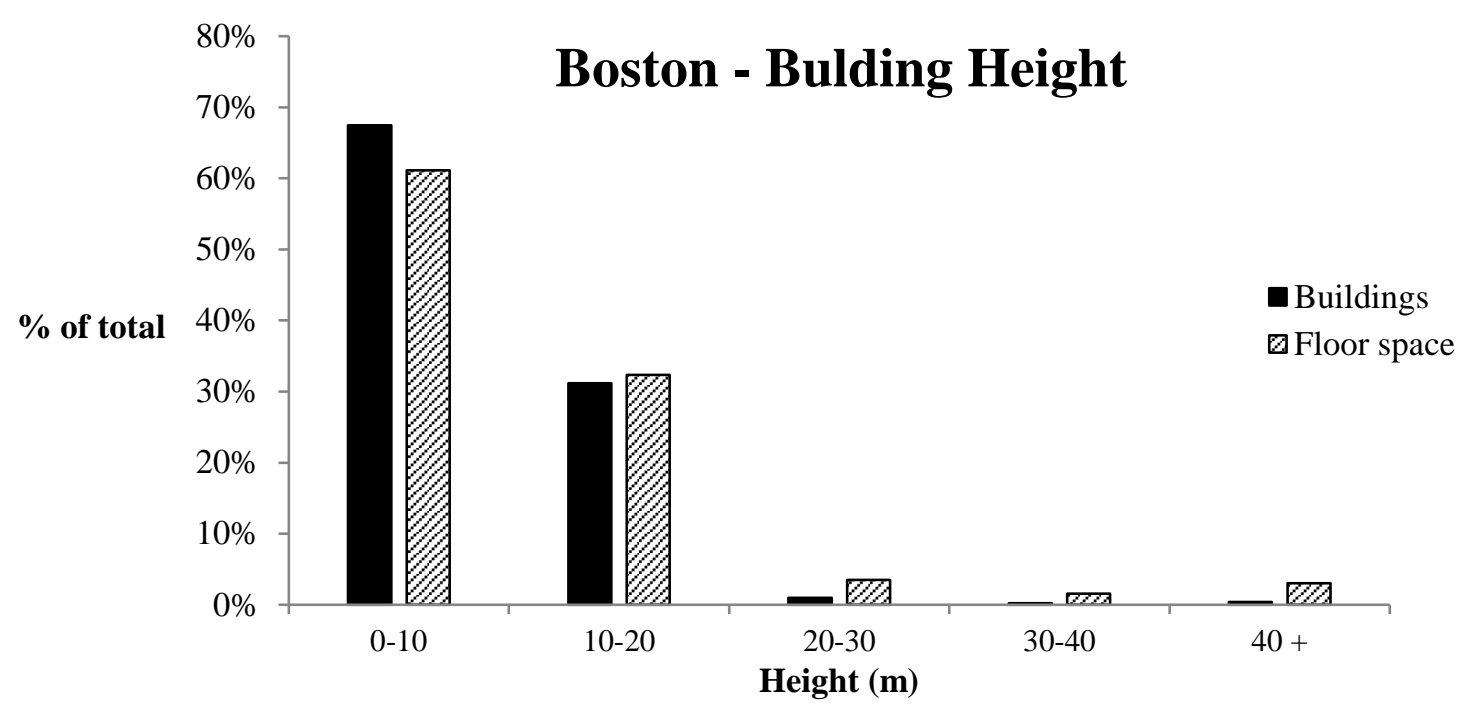

Figure 10. Number of buildings and building area in Boston for different building height ranges

Lastly, roof type is a natural indicator of UF suitability, since rooftop farms necessitate flat roofs. Only some of the tax data entries specify the roof type. Where no data on roof type was given, the roof type was assigned to a building probabilistically based on the representation of flat roofs in the general New England building stock. For commercial buildings this is a $25.2 \%$ probability according to the commercial building energy consumption survey ${ }^{25}$. As this data is lacking in the analogous residential survey, it is estimated as the percentage of buildings in our generated building data set with flat roofs, $21.3 \%$ (11735 of 55026 entries with roof data).

Table S43 shows the results of the different cutoff years, the height limit and the probabilistic assignment of roof top averaged over 100 runs.

\begin{tabular}{|c|c|c|c|c|}
\hline Cutoff Year & Building Space $\left(\mathbf{m}^{2}\right)$ & SD $\left(\mathbf{m}^{2}\right)$ & Building Space (acres) & SD (acres) \\
\hline 1900 & 1714149 & 34113 & o & \\
\hline 1910 & 942317 & 35025 & 233 & 9 \\
\hline 1920 & 672546 & 32088 & 166 & 8 \\
\hline 1930 & 512520 & 31076 & 127 & 8 \\
\hline 1940 & 440620 & 29107 & 109 & 7 \\
\hline 1950 & 406841 & 30041 & 101 & 7 \\
\hline 1960 & 334557 & 24713 & 83 & 6 \\
\hline 1970 & 253619 & 19861 & 63 & 5 \\
\hline 1980 & 215278 & 19309 & 53 & 5 \\
\hline 1990 & 156922 & 15967 & 39 & 4 \\
\hline 2000 & 104950 & 12094 & 26 & 3 \\
\hline
\end{tabular}

\section{Urban metabolism interactions}

This study accounts for three direct material/energy exchanges between farm and city: runoff retention, solid waste assimilation and building energy reductions.

\section{Runoff retention}

The engineering of the modern city has seen the channelizing, rerouting and burying of urban streams. The proliferation of impermeable surfaces throughout cities that prevent the penetration of the rainwater into the soil mean that much of this water is directed towards stormwater sewers, eventually bound for wastewater treatment instead of recharging groundwater aquifers or surface waters. The net effect is that when it rains, large volumes of water are unnecessarily sent for treatment or during intermittent heavy rain events, sewer capacity is exceeded and water from sewage pipes is vented to local surface waters ${ }^{26}$. If the stormwater is combined with sanitary water in a combined sewer, then heavy rain events can lead to the release of raw sewage when the sewers overflow in combined-sewer-overflow events $(\mathrm{CSO})^{27}$. Boston has over 235 miles of combined-sewers and $37 \mathrm{CSO}$ outfalls and is negatively impacted by CSO events during particularly intense or long rainfalls ${ }^{27}$. Since UF occasionally replaces impermeable area with soil that can either retain water for crop uptake or provide a hydraulic conduit between surface and groundwater it is important to model how the potential runoff mitigation provided by UF in Boston.

Here we consider to situations where UF implementation in Boston obviates runoff to the sewers: where UF replaces ground parking and where it is placed on buildings. We provide upper and lower bounds of runoff retention based on field studies of extensive green roofs. Lower and upper retention rates are taken as $50 \%^{28}$ and $74 \%{ }^{29}$, respectively. The same rates are applied to ground UF since they are also representative of runoff retention on permeable land ${ }^{26}$. This method ignores the heterogeneity of soil characteristics and resultant runoff retention, but as a basic estimate to gauge the impact of UF on Boston's hydrology it should suffice to identify whether the scale of these impacts are significant or miniscule. Moreover, this method ignores the ability for UF to reduce the prevalence of CSOs and toxic fallout from sewage releases. However, quantifying such impacts would require detailed information on CSO outfall locations and local pollution assimilation capacity that is beyond the scope of this exercise.

In assessing the GWP and land use impacts from avoided stormwater treatment, we use the ecoinvent 3.2 process 'Wastewater, unpolluted \{RoW\}| treatment of, capacity 5E91/year | Conseq, U' to model wastewater treatment in Boston. Using the aforementioned GWP and land use methods we calculate $0.293 \mathrm{~kg} \mathrm{CO}_{2} \mathrm{e}$ and $0.0260 \mathrm{~m}^{2}$ in avoided impacts per $\mathrm{m}^{3}$ avoided wastewater treatment.

Precipitation is taken as the 2000-2015 annual Boston average of $1.11 \mathrm{~m}^{30}$

Solid waste assimilation

We use primary data collected from the urban farms we have the following compost application rates: 
- $\quad$ Roof based UF: $2.8 \mathrm{~kg}$ compost $/ \mathrm{m}^{2}$

Ground based UF: $0.3 \mathrm{~kg}$ compost $/ \mathrm{m}^{2}$

Though the lower compost usage for roof based UF seems counterintuitive, it is a result of wind-related soil losses from green roofs and the need to supplement the expanded shale/concrete grow media with medium rich in nutrients and organic carbon. Ground-based UF is less affected by soil loss and tends to occur in a top-soil matrix rich in organic carbon and with greater nutrient sorption capacity, and hence, does not demand the same volume of nutrient/organic additions as the rooftop farms.

To convert from deposited compost to mass of avoided waste, we assume a mass loss of $32 \%$ from waste compost. This is a conservative estimate based on the open windrow composting of garden waste in the US ${ }^{31}$. Applying this factor we find that rooftop farms and ground-based UF can assimilate 4.1 and $0.4 \mathrm{~kg}$ organic waste $/ \mathrm{m}^{2}$, respectively. In modelling the environmental impacts of waste assimilation, we allocate the waste treatment and related avoided fertilizer production to the previous life-cycle of the waste, and the delivery of the waste to the UF site to the farms.

\section{Building Energy}

In modelling the potential interactions between a building's energy system and farm the following assumptions are made:

- No direct coupling of the building energy system and urban farm are made (e.g. no heat ventilation into the growing media to extend growing periods, etc.)

- Energy savings only apply to the floor directly below the roof. This will underestimate the energy savings to the entire building, since the attenuation of temperature shifts on the top floor will have a spillover effect on energy use on subsequent floors that diminishes with distance from the roof.

- We assume that the energy impacts of rooftop urban farming are similar to those from extensive green roofs.

- Effects at the city level are modeled in an additive manner, ignoring the multiplicative effect of large numbers of farms in proximity. This will underestimate total energy savings as reduced air conditioning use from an attenuated urban heat island effect are not counted here.

- Insulation values and heating fuels are assumed to be independent of other building characteristics (e.g. age, height, size, etc.) during the Monte Carlo simulations, as the building energy surveys lack data on relating these characteristics for the New England region.

Modeling building energy savings start first by characterizing the level of insulation on the building and the energy consumption per unit area for heating and cooling. Both of these parameters are taken from the residential and commercial building energy consumption surveys ${ }^{25,32}$. Heating and cooling energy intensities are assumed to be constant for all commercial and residential buildings in the city, while insulation levels are assigned probabilistically to each building at the start of each simulation. Likewise, the heating fuel and presence of air conditioning are assigned in the same manner to buildings that are lacking these data in the tax assessment survey. The prevalence of different heating types and air conditioning presence are also taken from the building energy surveys. Table S44 outlines these parameter values and their likelihood in the New England building stock.

\begin{tabular}{|c|c|c|c|c|}
\hline \multirow{2}{*}{ Parameter } & \multicolumn{2}{|l|}{ Residential } & \multicolumn{2}{|l|}{ Commercial } \\
\hline & Value & Probability & Value & Probability \\
\hline \multicolumn{5}{|c|}{ Energy Intensity } \\
\hline $\begin{array}{l}\text { Heating } \\
\text { Intensity }\end{array}$ & $352 \mathrm{MJ} / \mathrm{m}^{2} / \mathrm{a}^{*}$ & - & $465 \mathrm{MJ} / \mathrm{m}^{2} / \mathrm{a}$ & - \\
\hline $\begin{array}{c}\text { Cooling } \\
\text { Intensity }\end{array}$ & $6 \mathrm{MJ} / \mathrm{m}^{2} / \mathrm{a}^{*}$ & - & $51 \mathrm{MJ} / \mathrm{m}^{2} / \mathrm{a}$ & - \\
\hline \multicolumn{5}{|c|}{ Insulation Levels } \\
\hline Well & - & 0.36 & - & $0.36^{* * *}$ \\
\hline Adequate & - & 0.44 & - & $0.44^{* * *}$ \\
\hline Poor & - & 0.2 & - & $0.2^{* *}$ \\
\hline None & - & 0 & - & $0^{* * *}$ \\
\hline \multicolumn{5}{|c|}{ Air Conditioning Present } \\
\hline Yes & - & 0.76 & - & 0.62 \\
\hline No & - & 0.24 & - & 0.38 \\
\hline \multicolumn{5}{|c|}{ Heating Present } \\
\hline Yes & - & 1 & - & 0.87 \\
\hline No & - & 0 & - & 0.13 \\
\hline \multicolumn{5}{|l|}{ Heating Fuel } \\
\hline Electricity & - & 0.12 & - & 0.18 \\
\hline Natural Gas & - & 0.52 & - & 0.36 \\
\hline Fuel Oil & - & 0.32 & - & 0.46 \\
\hline Propane & - & 0.04 & - & 0 \\
\hline
\end{tabular}

(1)

** Not available in the commercial energy consumption survey. Assumed that same as residential values

To link UF with energy savings, a relation between insulation level and amount of cooling and heating attenuation is needed. Results from La Roche and Berardi's field work measuring energy savings of green roofs at different insulation thicknesses was useful in building this concordance ${ }^{34}$. In using their numbers we assume equivalent percentage savings for buildings in Chicago, US and Boston. Although Chicago has a continental climate with slightly warmer summers and cooler winters, the data adequate for the cursory analysis performed here. Table S45 outlines the concordance between the insulation levels here and the predicted energy savings from building-integrated UF.

\begin{tabular}{|l|l|l|l|}
\hline Table S45. Predicted energy savings at different insulation levels \\
\hline Insulation level from energy consumption survey & Insulation thickness from La Roche and Berardi $(\mathbf{m})^{34}$ & Heating attenuation (\%) & Cooling Attenuation (\%) \\
\hline Well & 0.20 & 0 & 7.5 \\
\hline Adequate & 0.10 & 0 & 7.5 \\
\hline Poor & 0.05 & 2.5 & 8 \\
\hline None & 0 & 7.5 & 15 \\
\hline
\end{tabular}

With these parameters in hand for each building, the UF related energy savings are estimated as the product of energy intensity, area and percentage attenuation. Embodied greenhouse gas impacts are taken from the Boston's own carbon footprint accounting since these represent the intensities for the local grid and fuel delivery systems ${ }^{35}$. Table $\mathrm{S} 46$ outlines emissions intensities for the different fuels used in Boston buildings for space conditioning.

\begin{tabular}{|l|l|}
\begin{tabular}{|l|l|}
\hline \multicolumn{2}{|l|}{ Table S46. Carbon intensities for different fuels in Boston } \\
\hline Energy source & kg CO2e/MJ supplied \\
\hline Electricity & 0.102 \\
\hline Natural Gas & 0.050 \\
\hline Fuel Oil & 0.070 \\
\hline Propane* & 0.050 \\
\hline
\end{tabular} Assumed equivalent to natural gas here. Minor role in energy system should not influence general results. \\
\hline
\end{tabular}




\section{City-wide optimization simulations}

In assessing the impacts of UF at the city level, all of the disparate pieces described in the preceding sections were tied together. A Python 2.7 script acts as a scaffolding with which to model the impacts of UF on Boston's food-borne GWP impacts and land use, and to model interactions between the urban farms and the city's energy and material metabolism. The script can optimize UF in Boston to maximize any one of three indicators at a time: GWP savings, land use savings and nutritional content. As many of the building parameters were assigned probabilistically, we run each optimization scenario 100 times in a Monte Carlo manner, randomly assigning UF suitability and building energy use characteristics. Despite the low number of runs, little variation is seen around the mean for the results, hinting at the suitability of our choice of simulation length. Requests for the script can be made through the corresponding author. Figure 11 outlines the algorithm.

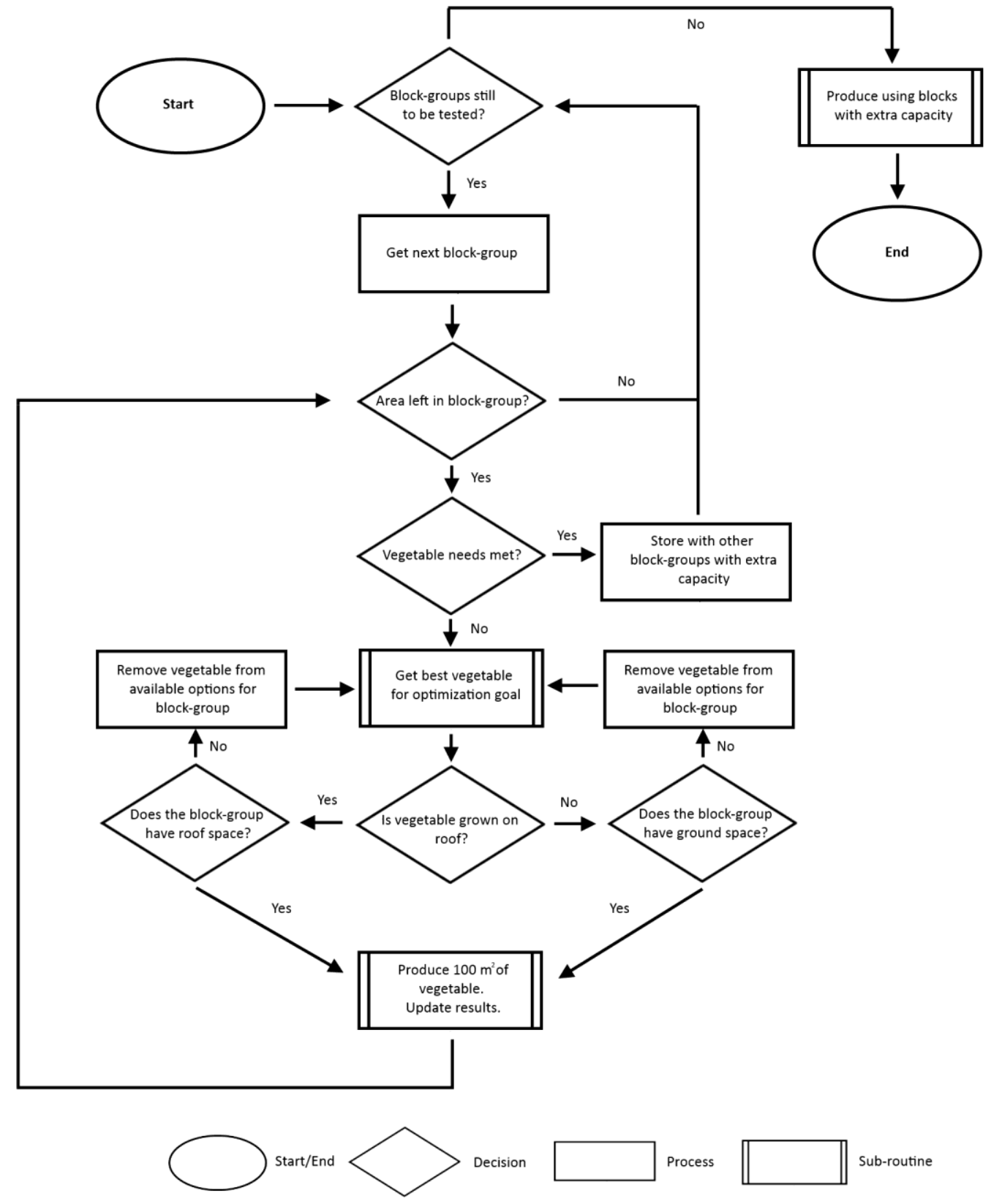

Figure 11. Optimization algorithm outline.

\section{GWP Optimization}

In optimizing the GWP impacts of the city's UF system, a 'greedy' algorithm is used. 'Greedy' algorithms work by picking items with the largest marginal benefit in terms of the parameter being optimized. In this case that means growing vegetables with the largest GWP impact reduction per unit area grown. Table S47 lists the UF produce with the largest reductions in GWP per area cultivated.

\begin{tabular}{|l|l|}
\hline \multicolumn{2}{|l|}{$\begin{array}{l}\text { Table S47. List of UF vegetables in order of decreasing } \\
\text { reductions in GWP impacts per } \mathbf{~}^{2} \text { planted }\end{array}$} \\
\hline Vegetable & Siting \\
\hline Kale & Ground \\
\hline Eggplant & Ground \\
\hline Green Beans & Ground \\
\hline Tomato & Ground \\
\hline Cucumber & Ground \\
\hline Bell Pepper & Ground \\
\hline Tomato & Roof \\
\hline Turnip & Ground \\
\hline Cucumber & Roof \\
\hline Lettuce & Ground \\
\hline Beet & Ground \\
\hline Carrot & Ground \\
\hline Scallion & Ground \\
\hline Bell Pepper & Roof \\
\hline Squash & Ground \\
\hline Collard Greens & Ground \\
\hline Cabbage & Ground \\
\hline Radish & Roof \\
\hline Lettuce & Roof \\
\hline Carrot & Roof \\
\hline Green Beans & Roof \\
\hline Kale & Roof \\
\hline Turnip & Roof \\
\hline Scallion & Roof \\
\hline
\end{tabular}

Each run of the algorithm cycles through all of Boston's block groups and performs the following sub-routine for each individual block group:

Block-group GWP optimizing sub-routine 
Is there area left in the block-group?

Yes: Are the block-group's needs met for all vegetables?

Yes: Store the block-group with others with extra capacity. End sub-routine.

No: Get the UF vegetable with the largest marginal GWP impact reduction.

Are all of the blocks needs met for this vegetable?

Yes: Remove vegetable from list of potential vegetables and get the next vegetable in the list.

No: Where is the vegetable grown?

Roof: Is there building space?

Yes: Produce $100 \mathrm{~m}^{2}$ of the vegetable (or remainder of roof space if less than $100 \mathrm{~m}^{2}$ left). Update results. Rerun sub-routine.

No: Remove vegetable from list of potential vegetables and attempt with next vegetable.

Ground: Is there ground space?

Yes: Produce $100 \mathrm{~m}^{2}$ of the vegetable (or remainder of ground space if less than $100 \mathrm{~m}^{2}$ left). Update results. Rerun sub-routine.

No: End sub-routine.

No: Remove vegetable from list of potential vegetables and attempt with next vegetable.

In this way each block-group will attempt to satisfy as much of its vegetable demands using those UF crops that minimize the GWP impacts of the blockgroup's residents. At the completion of a single cycle through all of Boston's block-groups, if there are block-groups that are able to satiate there vegetable demands while having surplus space, a separate sub-routine is run on those blocks:

\section{City GWP optimizing sub-routine}

\section{Is there area left in the block?}

Yes: Are all of the city's needs met for all vegetables?

Yes: End sub-routine.

No: Get the UF vegetable with the largest marginal GWP impact reduction.

Are all of the city's needs met for this vegetable?

Yes: Remove vegetable from list of potential vegetables and get the next vegetable in the list.

No: Where is the vegetable grown?

Roof: Is there building space?

Yes: Produce $100 \mathrm{~m}^{2}$ of the vegetable (or remainder of roof space if less than $100 \mathrm{~m}^{2}$ left). Update results. Rerun sub-routine.

No: Remove vegetable from list of potential vegetables and attempt with next vegetable.

Ground: Is there ground space?

Yes: Produce $100 \mathrm{~m}^{2}$ of the vegetable (or remainder of ground space if less than $100 \mathrm{~m}^{2}$ left). Update results. Rerun sub-routine.

No: End sub-routine.

No: Remove vegetable from list of potential vegetables and attempt with next vegetable.

This sub-routine is run on all of the block-groups with auxiliary space until all are exhausted or the city's vegetable needs are met. This algorithm can be run with additive or subtractive UF space estimates, including or excluding parking.

In determining the block-group and city-wide vegetable demands we use the 2010 LAFA data for average demands at the household prior to household wastage and multiply by the population for each block-group. This assumes that wastage from the urban farms is negligible, which was observed during in the field while working with the case farms. We do not attempt to satiate the needs for all vegetables listed in the USDA LAFA data ${ }^{2}$, but only those that UF produces or where UF crops act as reasonable substitutes. Table S48 shows the average intake of relevant vegetables from the LAFA data.

\begin{tabular}{|c|c|c|c|c|}
\hline Vegetable & Raw LAFA (lb/a) & Per capita demand of UF crop $(\mathrm{kg} / \mathrm{a})$ & Total Boston Demand (kg/a) & Fraction of total vegetables \\
\hline Beans & & 2.31 & 1424548.816 & 0.045155 \\
\hline Fresh & 1.44 & & & \\
\hline Canned & 2.07 & & & \\
\hline Frozen & 1.58 & & & \\
\hline Beet* & 0.24 & 0.11 & 68551.87793 & 0.002173 \\
\hline Bell Pepper & 8.77 & 3.98 & 2456900.259 & 0.077879 \\
\hline Cabbage & & 2.90 & 1786523.718 & 0.056629 \\
\hline Fresh & 5.96 & & & \\
\hline Canned & 0.41 & & & \\
\hline Carrots & & 3.83 & 2361388.616 & 0.074851 \\
\hline Fresh & 7.14 & & & \\
\hline Canned & 0.53 & & & \\
\hline Frozen & 0.76 & & & \\
\hline Collard Greens & 0.51 & 0.23 & 143684.5788 & 0.004555 \\
\hline Cucumbers & & 3.28 & 2023733.81 & 0.064148 \\
\hline Fresh & 5.81 & & & \\
\hline Canned & 1.41 & & & \\
\hline Eggplant & 0.53 & 0.24 & 147267.9982 & 0.004668 \\
\hline Kale & & 0.89 & 549605.5795 & 0.017421 \\
\hline Kale & 0.24 & & & \\
\hline Spinach & 1.27 & & & \\
\hline Frozen Spinach & 0.45 & & & \\
\hline Lettuce & & 10.52 & 6484207.704 & 0.205537 \\
\hline Leaf & 13.52 & & & \\
\hline Romaine & 9.62 & & & \\
\hline Radish & 0.38 & 0.17 & 106540.9844 & 0.003377 \\
\hline Scallion & 0.24 & 0.11 & 68551.87793 & 0.002173 \\
\hline Squash & & 3.13 & 1931088.178 & 0.061212 \\
\hline Squash & 3.40 & & & \\
\hline Pumpkin & 3.49 & & & \\
\hline Tomato & & 19.34 & 11926557.87 & 0.378048 \\
\hline Fresh & 15.2 & & & \\
\hline Canned & 27.4 & & & \\
\hline Turnip* & 0.24 & 0.11 & 68551.87793 & 0.002173 \\
\hline
\end{tabular}

Land use optimization 
This method is identical to the GWP impact algorithm except that UF crops are now listed in order of their ability to reduce land use. Table S49 shows the list of vegetables when ordered in this manner.

\begin{tabular}{|l|l|}
\hline \multicolumn{2}{|l|}{$\begin{array}{l}\text { Table S49. List of UF vegetables in order of decreasing } \\
\text { reductions in land use per } \mathbf{~}^{2} \text { planted }\end{array}$} \\
\hline Vegetable & Siting \\
\hline Green Beans & Ground \\
\hline Radish & Roof \\
\hline Kale & Ground \\
\hline Cucumber & Roof \\
\hline Green Beans & Roof \\
\hline Turnip & Ground \\
\hline Tomato & Roof \\
\hline Turnip & Roof \\
\hline Bell Pepper & Roof \\
\hline Kale & Roof \\
\hline Squash & Ground \\
\hline Cucumber & Ground \\
\hline Lettuce & Roof \\
\hline Carrot & Roof \\
\hline Cabbage & Ground \\
\hline Scallion & Roof \\
\hline Beet & Ground \\
\hline Tomato & Ground \\
\hline Collard Greens & Ground \\
\hline Carrot & Ground \\
\hline Scallion & Ground \\
\hline Bell Pepper & Ground \\
\hline Eggplant & Ground \\
\hline Lettuce & Ground \\
\hline
\end{tabular}

\section{Nutritional Optimization}

In optimizing for nutrition, the algorithm is moved away from a greedy mode. This is because the boundary for knowing when to stop producing the bestoption vegetable in the greedy mode is the demand for that crop at the block-group or city level. In the nutritional optimizing algorithm we are in fact attempting to change the boundary condition, that is, the amount of certain foods consumed, eliminating this indicators candidacy for this role. Instead we randomly pick vegetables in a manner that reflect the consumption patterns of the average consumer, whilst trying provide as much nutrition as possible, beyond their typical demands, aiming to satisfy the nutritional needs as outlined by the USDA guidelines ${ }^{1}$.

USDA guidelines provide recommended intakes for four vegetable types: starchy, dark green, red and orange and other. Here we focus on the last three since none of the vegetables in the first group are produced by any of the case farms. The nutritional algorithm has two variants. The first attempts to produce as much vegetables as possible and satiate the entire vegetable demands of the block-group (and city using surplus land). The second version attempts to close the gap between current consumption and USDA guidelines. In both versions the vegetable group with the largest distance to target is always prioritized, so that in the first variant it will end up producing nearly the same fraction of USDA guidelines, while the second may or may not end up satisfying all groups to the same extent. Importantly, the second variant models a situation where UF is not substituting conventional supply chains, but supplementing, and hence, no crediting for avoided conventional production is accounted for.

Vegetable demands are taken from the USDA guidelines for different sexes and age groups. The deficiency is taken as the difference between the USDA guidelines and usual daily intake from the NHANES data ${ }^{36}$. Individual nutritional demands and deficiencies are then combined with census demographics data for each block-group to get the total demands at block-group and city level. Table S50 shows the nutritional demands and deficits for different demographics.

\begin{tabular}{|c|c|c|c|c|c|c|c|c|c|c|c|c|c|c|c|c|c|}
\hline \multirow[b]{3}{*}{ Vegetable Category } & \multirow[b]{3}{*}{ Unit } & \multirow{2}{*}{\multicolumn{8}{|c|}{ Males }} & \multicolumn{8}{|l|}{ Females } \\
\hline & & Age & & & & & & & & Age & & & & & & & \\
\hline & & $1-3$ & $4-8$ & $9-13$ & $14-18$ & $19-30$ & $31-50$ & $51-70$ & $71+$ & $1-3$ & $4-8$ & $9-13$ & $14-18$ & $19-30$ & $31-50$ & $51-70$ & $71+$ \\
\hline $\begin{array}{l}\text { Dark Greens } \\
\end{array}$ & cup eq. & $0.5(0.5)$ & $1(1)$ & $1.5(1.5)$ & $2(1.3)$ & $2.5(1.8)$ & $2.5(1.8)$ & $2(0.6)$ & $2(1.3)$ & $1(1)$ & $1.5(1.5)$ & $1.5(1.5)$ & $1.5(0.8)$ & $\begin{array}{ll}1.5(0.8) \\
\end{array}$ & $1.5(0.8)$ & $1.5(0.1)$ & $1.5(0.8)$ \\
\hline Red and Orange & cup eq. & $2.5(1.1)$ & $3(0.9)$ & $5.5(3.4)$ & $6(3.6)$ & $7(3.5)$ & $7(3.5)$ & $6(3.2)$ & $6(3.2)$ & $\begin{array}{r}2.5 \\
(1.1)\end{array}$ & $3(1.6)$ & $5.5(3.4)$ & $5.5(3.4)$ & $5.5(2.7)$ & $5.5(2.7)$ & $5.5(2.7)$ & $5.5(2.7)$ \\
\hline Other & cup eq. & $1(1.3)$ & $3.5(1.4)$ & $5(3.6)$ & $6(3.9)$ & $7(3.8)$ & $7(3.8)$ & $6(0.6)$ & $6(2.5)$ & $2(0.6)$ & $3(1.6)$ & $5(2.9)$ & $5(2.9)$ & $5(1.5)$ & $5(0.8)$ & $5(0.1)$ & $5(1.5)$ \\
\hline
\end{tabular}

Taking the institution adjusted population of Boston of 616,602 in 2010 and the demographic spread, we estimate the city-wide nutritional demands as $5.68 \times 10^{7}, 1.83 \times 10^{8}$ and $1.75 \times 10^{8}$ cup eq. of dark green, red and orange, and other vegetables, respectively. City-wide nutritional deficits are estimated as $1.96 \times 10^{7}, 6.64 \times 10^{8}$ and $2.62 \times 10^{7}$ cup eq. for dark green, red and orange, and other vegetables, respectively.

Table S51 summarizes the UF crops in terms of their vegetable type and the amount of nutritional units supplied per area planted.

\begin{tabular}{|l|l|l|r|}
\hline \multicolumn{4}{|l|}{ Table S51. UF Crops and their nutritional properties } \\
\hline Vegetable & USDA Category & Siting & Cup eq./ $\mathbf{m}^{2}$ \\
\hline Beans & Other & Ground & 28 \\
\hline Beet & Other & Ground & 17 \\
\hline Bell Pepper & Red and Orange & Ground & 19 \\
\hline Cabbage & Other & Ground & 52 \\
\hline Carrots & Red and Orange & Ground & 13 \\
\hline Collard Greens & Dark Green & Ground & 6 \\
\hline Cucumbers & Other & Ground & 28 \\
\hline Eggplant & Other & Ground & 28 \\
\hline Kale & Dark Green & Ground & 36 \\
\hline Lettuce & Other & Ground & 7 \\
\hline Squash & Other & Ground & 22 \\
\hline Tomato & Red and Orange & Ground & 17 \\
\hline Turnip & Other & Ground & 27 \\
\hline Scallion & Other & Ground & 8 \\
\hline Beans & Other & Roof & 10 \\
\hline Bell Pepper & Red and Orange & Roof & 20 \\
\hline Carrots & Red and Orange & Roof & 13 \\
\hline Cucumbers & Other & Roof & 44 \\
\hline Kale & Dark Green & Roof & 10 \\
\hline Lettuce & Other & Roof & 7 \\
\hline Radish & Other & Roof & 25 \\
\hline Scallion & Other & Roof & 9 \\
\hline
\end{tabular}




\begin{tabular}{|l|l|l|r|}
\hline Tomato & Red and Orange & Roof & 28 \\
\hline Turnip & Other & Roof & 14 \\
\hline
\end{tabular}

Block-group nutritional optimization sub-routine

Is there area left in the block-group?

Yes: Are the block-group's nutritional demands (or deficit) met?

Yes: Store the block-group with others with extra capacity. End sub-routine.

No: Determine the vegetable type with largest distance to gap.

Is there building space?

Yes: Randomly choose vegetable from amongst those within the vegetable category that are grown on buildings, with probability based on usual intake rates. Produce $100 \mathrm{~m}^{2}$ of the vegetable (or remainder of roof space if less than $100 \mathrm{~m}^{2}$ left). Update results. Rerun sub-routine.

No: Randomly choose vegetable from amongst those within the vegetable category that are grown on the ground, with probability based on usual intake rates. Produce $100 \mathrm{~m}^{2}$ of the vegetable (or remainder of roof space if less than $100 \mathrm{~m}^{2}$

No: End sub-routine. left). Update results. Rerun sub-routine.

After all block-groups are given the chance to produce for themselves, those with surplus growing area attempt to produce to satisfy Boston's nutritional needs.

City nutritional optimization sub-routine

Is there area left in the block-group?

Yes: Are the city's nutritional demands (or deficit) met?

Yes: End sub-routine.

No: Determine the vegetable type with largest distance to gap at the city level.

Is there building space?

Yes: Randomly choose vegetable from amongst those within the vegetable category that are grown on buildings, with probability based on usual intake rates. Produce $100 \mathrm{~m}^{2}$ of the vegetable (or remainder of roof space if less than $100 \mathrm{~m}^{2}$ left). Update results. Rerun sub-routine.

No: Randomly choose vegetable from amongst those within the vegetable category that are grown on the ground, with probability based on usual intake rates. Produce $100 \mathrm{~m}^{2}$ of the vegetable (or remainder of roof space if less than $100 \mathrm{~m}^{2}$

No: End sub-routine.

eft). Update results. Rerun sub-routine.

In the same manner is the GWP and land use sub-routines, this algorithm can be used with additive and subtractive UF land use estimates.

\section{UF Revenue}

Crop prices are taken from consumer expenditure data (averaged over the available years) ${ }^{37}$ or from USDA retail reports on specialty crops ${ }^{38}$. Table 49 outlines the crop prices used here in current US dollars.

\begin{tabular}{|l|l|l|}
\hline \multicolumn{3}{|l|}{ Table S52. Crop prices } \\
\hline Vegetable & USD/kg & Source \\
\hline Beans & 3.20 & Consumer Expenditure \\
\hline Beet & 2.19 & USDA \\
\hline Cabbage & 1.42 & Consumer Expenditure \\
\hline Carrots & 1.72 & Consumer Expenditure \\
\hline Collard Greens & 2.13 & USDA \\
\hline Cucumbers & 2.85 & USDA \\
\hline Eggplant & 3.01 & USDA \\
\hline Iceberg & 2.28 & Consumer Expenditure \\
\hline Kale & 2.28 & USDA \\
\hline Peppers & 5.38 & Consumer Expenditure \\
\hline Radish & 3.51 & USDA \\
\hline Scallion & 1.22 & USDA \\
\hline Squash & 1.86 & USDA \\
\hline Tomato & 3.71 & Consumer Expenditure \\
\hline Turnip & 2.19 & USDA \\
\hline
\end{tabular}

When the city only produces for its residents, the above algorithms are unaltered, and the revenue from block-group trade is calculated and recorded along with all of the environmental and nutritional results.

The only shift to the algorithm is when the block group starts exporting the conurbation. A crude method would simply produce the most profitable crop, but this would actually lead to a glut of one or two crops on the market, leading to a crash in prices. To avoid this, the city's extra space is allocated to crops based on the usual demand for the crop according to the USDA LAFA data (see rightmost column of Table 45). The above algorithms remain unaltered from the above cases with the exception of a sub-routine that is run at the end on all block-groups with surplus land:

Is there area left in the block-group?

Yes: Randomly select vegetable based on usual intake probability.

Is there suitable UF space to grow the crop (either roof or ground)?

Yes: Grow $100 \mathrm{~m}^{2}$ (or available area) of that vegetable and update results. Rerun sub-routine.

No: End sub-routine. 
a)

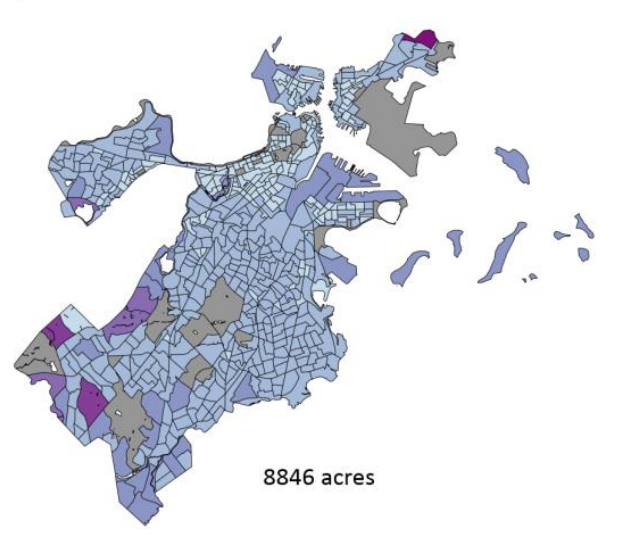

b)

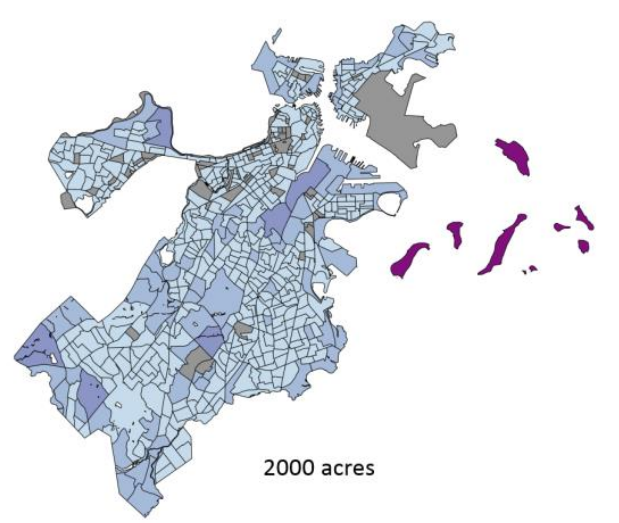

c)

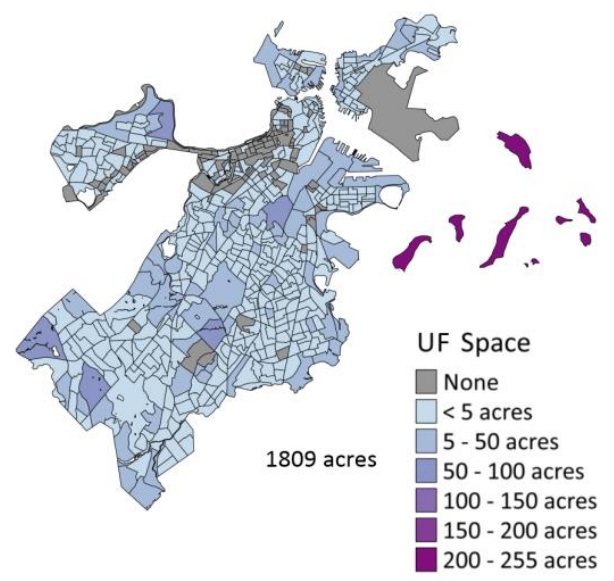

d)

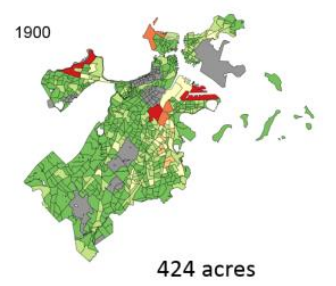

424 acres

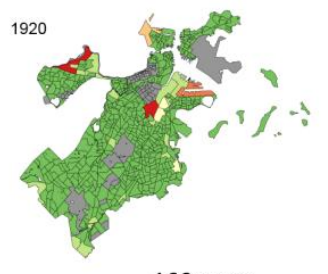

166 acres
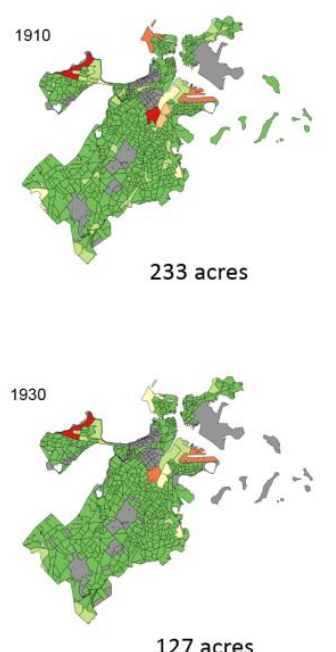

127 acres

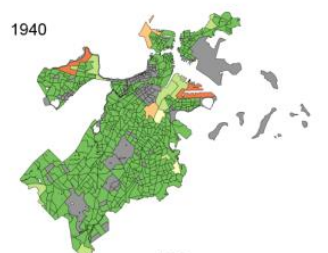

109 acres

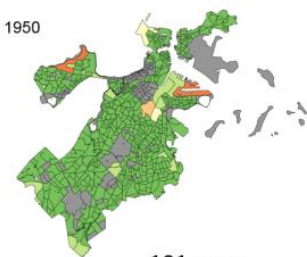

101 acres
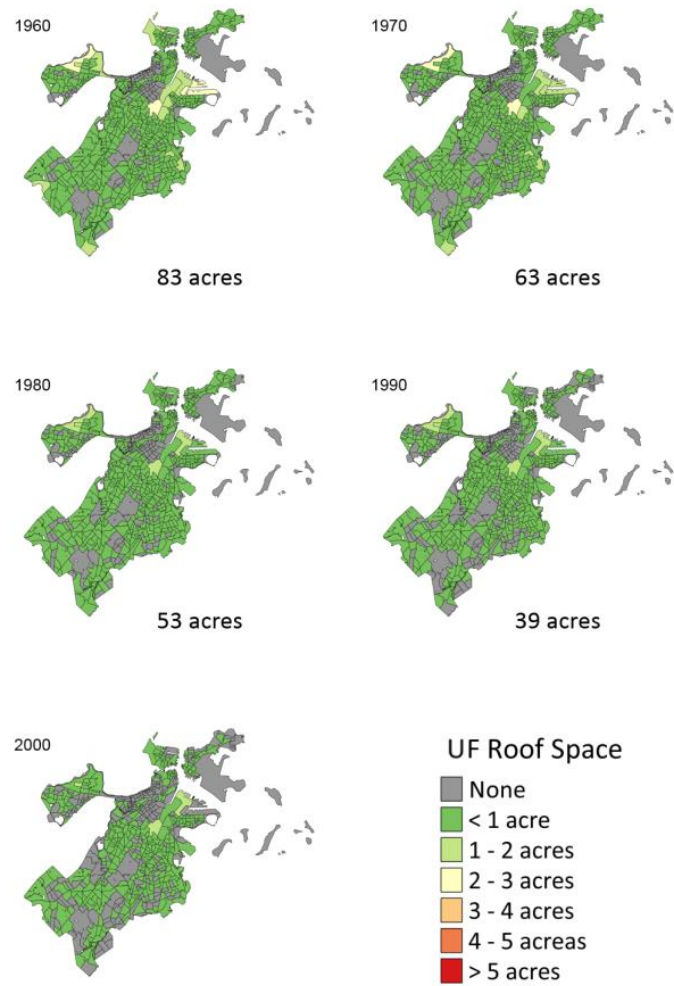

UF Roof Space

$\square$ None

$<1$ acre
$1-2$ acres

2 - 3 acres

$3-4$ acres

$4-5$ acrea

Figure 12. UF space results for (a) subtractive, (b) additive, (c) additive minus parking and (d) rooftop
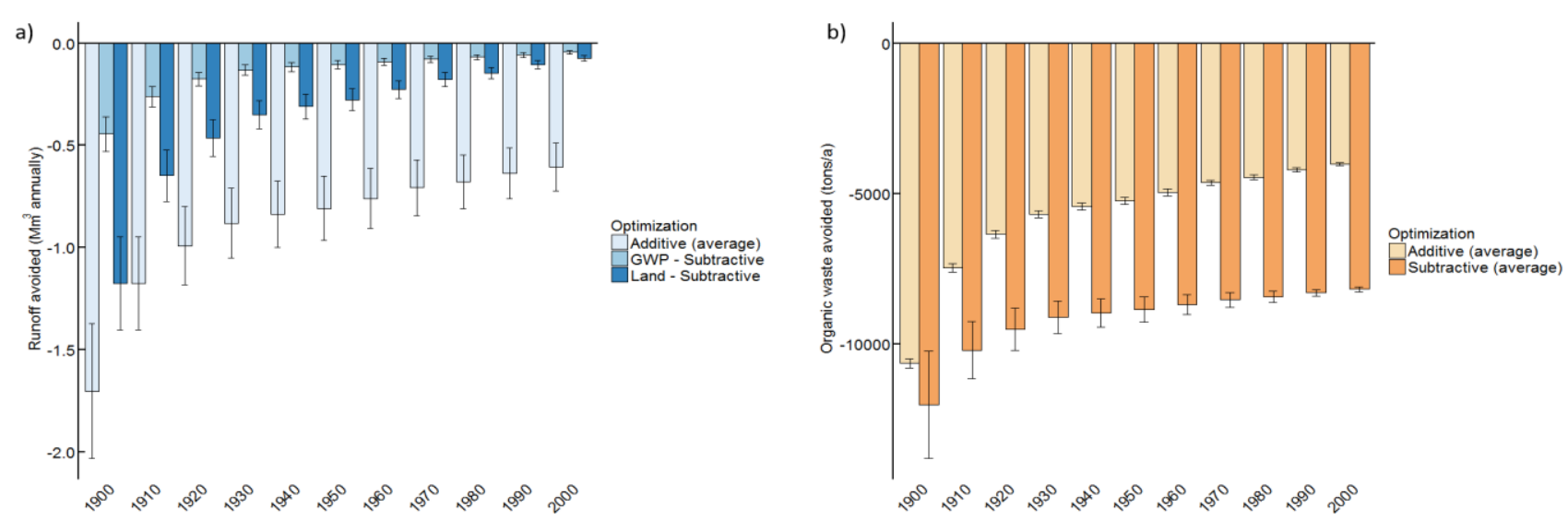

Figure 13. (a) avoided runoff results for the different scenarios and years . Additive results for both land and GWP are averaged due to similarity. (b) Organic waste uptake from UF averaged for both optimizations due to similarity. 
Raw Results

Table S53 - GWP optimization where UF space was estimated with an additive method.

Table S54 - GWP optimization where UF space was estimated with an additive method, but excluding parking.

Table S55 - GWP optimization where UF space was estimated with a subtractive method.

Table S56 - GWP optimization where UF space was estimated with a subtractive method with vegetable exporting

Table S57 - Land use optimization where UF space was estimated with an additive method.

Table S58 - Land use optimization where UF space was estimated with an additive method, but excluding parking.

Table S59 - Land use optimization where UF space was estimated with a subtractive method.

Table S60 - Land use optimization where UF space was estimated with a subtractive method with vegetable exporting

Table S61 - Nutritional needs optimization where UF space was estimated with an additive method.

Table S62 - Nutritional needs optimization where UF space was estimated with an additive method, but excluding parking.

Table S63 - Nutritional deficit optimization where UF space was estimated with an additive method, but excluding parking.

Table S64 - Nutritional needs optimization where UF space was estimated with a subtractive method.

Table S65 - Nutritional needs optimization where UF space was estimated with a subtractive method with vegetable exporting
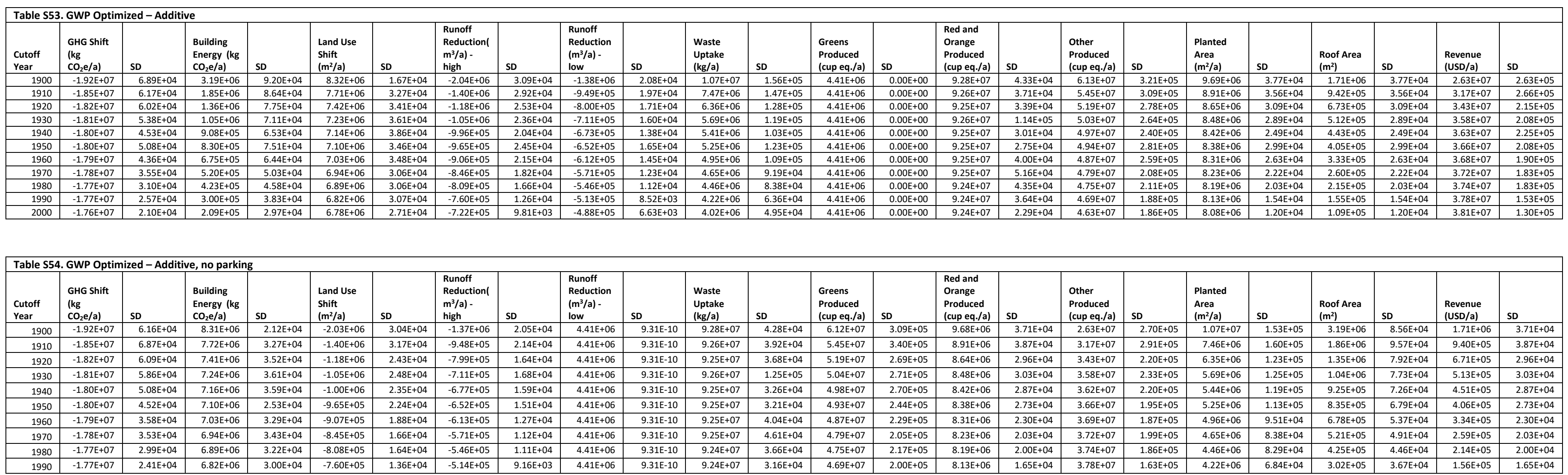


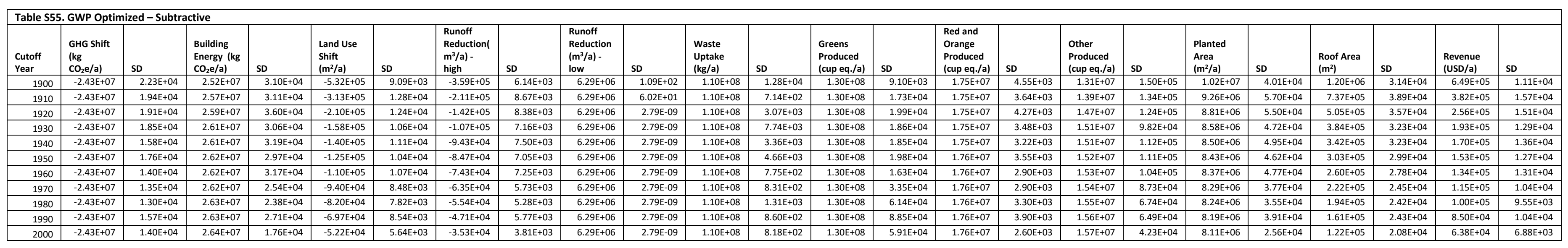

\begin{tabular}{|c|c|c|c|c|c|c|c|c|c|c|c|c|c|c|c|c|c|c|c|c|c|c|c|c|c|c|}
\hline & & & & & & & & & & & & & & & & & & & & & & & & & & \\
\hline $\begin{array}{l}\text { Cutoff } \\
\text { Year }\end{array}$ & $\begin{array}{l}\text { HGG Shift } \\
\text { (kg } \\
{ }^{0} \mathrm{O}_{2} / \mathrm{a}\end{array}$ & & $\begin{array}{l}\text { Building } \\
\text { Energy } \\
\text { (kg } \\
\text { cko } 2 \text { e/a) }\end{array}$ & & $\begin{array}{l}\text { land Use } \\
\text { Shitit } \\
\left(m^{2} / a\right)\end{array}$ & & $\begin{array}{l}\text { Runoff } \\
\text { Reductio } \\
\text { nnm } m \text { (m/a). } \\
\text { high }\end{array}$ & & $\begin{array}{l}\text { Runoff } \\
\text { Reductio } \\
n\left(m^{3} / 2\right)- \\
\text { low }\end{array}$ & SD & $\begin{array}{l}\text { Waste } \\
\text { Uptateke } \\
(\mathrm{k} / \mathrm{g} / \mathrm{a}\end{array}$ & & $\begin{array}{l}\text { Sreens } \\
\text { Produced } \\
\text { (cupp } \\
\text { eq./f) }\end{array}$ & & $\begin{array}{l}\text { Red and } \\
\text { orange } \\
\text { Produced } \\
\text { lcup } \\
\text { eq./a) }\end{array}$ & & \begin{tabular}{|l|} 
Other \\
Produced \\
(cup \\
eq./a) \\
\end{tabular} & & $\begin{array}{l}\text { Planted } \\
\text { Area } \\
(\mathrm{m} / 2 / 2\end{array}$ & & $\begin{array}{l}\text { Roof } \\
\text { Areaa }\end{array}$ & & $\begin{array}{l}\text { venuu } \\
\text { sDo(a) }\end{array}$ & & $\begin{array}{l}\text { Export } \\
\text { (UUDD/a) }\end{array}$ & \\
\hline & $-5.93 \mathrm{E}+07$ & $1.13 \mathrm{E}+05$ & $4.10 \mathrm{~F}+07$ & $6.92 E+04$ & $-1.40 E+06$ & $2.72 E+04$ & \begin{tabular}{|l|}
$-9.49 E+05$ \\
\end{tabular} & $1.84 \mathrm{E}+04$ & $1.90 \mathrm{E}+07$ & $1.911+05$ & $2.966+08$ & \begin{tabular}{|l|}
$7.53 \mathrm{E}+05$ \\
\end{tabular} & $3.08 E+08$ & $7.06 \mathrm{E}$ & $3.74 E+07$ & $3.32 \mathrm{E}+04$ & $1.69 \mathrm{E}+08$ & $4.36 E+05$ & $2.31 \mathrm{E}+07$ & \begin{tabular}{|l|}
$1.37 \mathrm{E}+05$ \\
\end{tabular} & $3.19 E+06$ & $8.09 E+04$ & $1.71 E+06$ & \begin{tabular}{|c|c|}
$3.32 \mathrm{E}+04$ \\
\end{tabular} & $1.56 E+08$ & $4.60 \mathrm{E}+05$ \\
\hline 1910 & $-5.77 \leftleftarrows+07$ & $1.14 \mathrm{E}+05$ & $\begin{array}{l}4.17 \mathrm{E}+07 \\
\end{array}$ & $6.35 E+04$ & \begin{tabular}{|l|l|}
$-7.699+05$ \\
\end{tabular} & $2.97 F+04$ & $\begin{array}{ll}-5.20 \mathrm{E}+05 \\
\end{array}$ & $2.00 E+04$ & \begin{tabular}{|l|l}
$1.88 \mathrm{E}+07$ \\
\end{tabular} & \begin{tabular}{|l|}
$2.00 E+05$ \\
\end{tabular} & $2.86 \mathrm{E}+08$ & $8.29 E+05$ & $3.02 E+08$ & $7.07 \mathrm{E}+05$ & \begin{tabular}{|l|l|}
$3.67 \mathrm{E}+07$ \\
\end{tabular} & $3.62 E+04$ & $1.63 E+08$ & $\begin{array}{l}4.68 E \mathrm{E}+05 \\
\end{array}$ & $1.99 E+07$ & $1.50 \mathrm{E}+05$ & $1.85 E+06$ & \begin{tabular}{ll|l|l}
$8.78 E+04$ & \\
\end{tabular} & \begin{tabular}{|l|}
$9.399 \mathrm{E}+05$ \\
\end{tabular} & \begin{tabular}{|l|l|l}
$3.62 E+004$ \\
\end{tabular} & $1.49 E+08$ & $\begin{array}{l}4.74 E+05 \\
\end{array}$ \\
\hline 1920 & $-5.72 E+07$ & $1.07 E+05$ & $4.19 \mathrm{E}+07$ & $8.24 E+04$ & $-5.52 E+05$ & $2.41 E+04$ & $\begin{array}{l}-3.73 \mathrm{E}+05 \\
\end{array}$ & $\begin{array}{ll}1.63 \mathrm{E}+04 \\
\end{array}$ & \begin{tabular}{|l|l|}
$1.86 E+07$ \\
\end{tabular} & $1.95 E+05$ & $2.83 \mathrm{E}+08$ & \begin{tabular}{ll|}
$7.011+05$ \\
\end{tabular} & $3.011 \mathrm{E}+08$ & $7.98 \varepsilon+05$ & \begin{tabular}{|l|l|}
$3.64 E \mathrm{E}+07$ \\
\end{tabular} & $2.95 E+04$ & 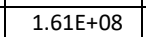 & $4.23 \mathrm{E}+05$ & $\begin{array}{l}1.88 E+07 \\
\end{array}$ & \begin{tabular}{ll|l}
$1.22 E+05$ \\
\end{tabular} & $\begin{array}{ll}1.36 \mathrm{E}+06 \\
\end{array}$ & $7.38 \mathrm{E}+04$ & $6.74 E+05$ & \begin{tabular}{ll|}
$2.955+04$ \\
\end{tabular} & $1.46 E+08$ & $4.38 E+05$ \\
\hline 1930 & $-5.68 E+07$ & $1.04 E+05$ & $4.20 E+07$ & $7.35 E+04$ & $-4.222 E+05$ & $2.52 E+04$ & $-2.855+05$ & $1.70 E+04$ & $1.86 \mathrm{E}+07$ & +05 & $2.81 E+08$ & $6.89 \mathrm{C}+05$ & $2.996+08$ & $7.88 \varepsilon+05$ & \begin{tabular}{|l|l|}
$3.63 E+07$ \\
\end{tabular} & $3.08 E+04$ & \begin{tabular}{|l|l|l|l|}
$1.599 \mp+88$ \\
\end{tabular} & $4.42 E+05$ & $1.82 E+07$ & $1.27 \mathrm{E}+05$ & $1.05 E+06$ & $7.39 \mathrm{E}+04$ & $5.15 E+05$ & $3.08 \mathrm{E}+04$ & $1.44 E+08$ & $4.51 E+05$ \\
\hline & $-5.67 \mathrm{E}+07$ & $1.06 E+05$ & 4.2. & $7.36 E+04$ & $-3.655+05$ & $2.46 E+04$ & $\begin{array}{l}-2.47 飞+05 \\
\end{array}$ & $1.66 \mathrm{E}+04$ & \begin{tabular}{|l|l}
$1.85 E+07$ \\
\end{tabular} & $2.24 E+05$ & $2.80 E+08$ & $7.055+05$ & $2.99 \varepsilon+08$ & \begin{tabular}{|l|l|}
$7.38 E+05$ \\
\end{tabular} & \begin{tabular}{|l|}
$3.62 E+07$ \\
\end{tabular} & $3.00 \mathrm{E}+04$ & \begin{tabular}{|l|l|}
$1.599+08$ \\
\end{tabular} & $4.34 E+05$ & $1.795+07$ & $1.24 E+05$ & \begin{tabular}{|l|l|}
$9.14 E+05$ \\
\end{tabular} & $6.90 E+04$ & $4.46 E+05$ & $3.00 E+04$ & $1.44 E+08$ & $4.33 E+05$ \\
\hline & $-5.65 E+07$ & $1.05 E+05$ & & $6.10 €+04$ & $-3.29 E+05$ & $2.59 E+04$ & $-2.22 E+05$ & $1.75 E+04$ & $1.85 \mathrm{E}+07$ & $2.13 E+05$ & $2.79 E+08$ & $7.13 \mathrm{E}+05$ & $2.98 E+08$ & $7.68 \mathrm{~F}+05$ & $3.611+07$ & $3.16 \mathrm{E}+04$ & \begin{tabular}{|c|}
$1.58 \mathrm{E}+08$ \\
\end{tabular} & $4.03 E+05$ & $1.77 \leftleftarrows+07$ & \begin{tabular}{|l|}
$1.31 E+05$ \\
\end{tabular} & $8.17 €+05$ & $7.97 €+04$ & $4.02 E+05$ & $3.16 €+04$ & $1.43 E+08$ & $4.111+05$ \\
\hline 196 & $-5.64 E+07$ & 8.1 & & $7.32 E+04$ & $-2.77 \mathrm{~F}+05$ & $2.00 E+04$ & $-1.87 \mathrm{~F}+05$ & $1.35 E+04$ & $1.85 \mathrm{E}+07$ & 05 & $2.78 \mathrm{E}+08$ & 5.73 & $2.98 E+08$ & $7.796+05$ & $3.61 \mathrm{E}+07$ & $2.44 \mathrm{E}+04$ & \begin{tabular}{|c|}
$1.58 \mathrm{E}+08$ \\
\end{tabular} & $3.511+05$ & $1.74 \mathrm{E}+07$ & \begin{tabular}{|l|}
$1.01 E+05$ \\
\end{tabular} & $6.83 E+05$ & $5.60 €+04$ & $3.38 E+05$ & $2.44 E+04$ & $1.43 E+08$ & $3.411+05$ \\
\hline 1970 & $-5.62 \mathrm{E}$ & 8.4 & 4.2 & 5.63 & -2.12 & $1.92 E+04$ & -1.43 & 1.3 & $1.84 \mathrm{E}$ & 05 & $2.77 F+08$ & 5.4 & $2.98 E+08$ & $6.966+05$ & $3.60 \mathrm{E}+07$ & $2.34 \mathrm{E}+04$ & \begin{tabular}{|c|}
$1.57 E+08$ \\
\end{tabular} & $3.22 E+05$ & $1.71 E+07$ & \begin{tabular}{|c|}
$9.69 \mathrm{E}+04$ \\
\end{tabular} & $5.18 E+05$ & $5.32 \leftleftarrows+04$ & $2.59 E+05$ & $2.34 E+04$ & $1.42 E+08$ & $3.211+05$ \\
\hline 1980 & -5.61 & 7.74 & 4.2 & $6.5 \mathrm{C}$ & -1.7 & 1.55 & -1.1 .1 & 1.0 & 1.84 & 5 & 2.7 & 4. & 2. & 6.74 & 3.66 & $1.89 \mathrm{E}+04$ & \begin{tabular}{|c|}
$1.57 \mathrm{E}+08$ \\
\end{tabular} & $2.75 E+05$ & $1.69 \mathrm{E}+07$ & \begin{tabular}{|l|}
$7.80 E+04$ \\
\end{tabular} & $4.29 E+05$ & $4.48 E+04$ & $2.15 E+05$ & $1.89 €+04$ & $1.41 E+08$ & $2.83 E+05$ \\
\hline 1990 & $-5.60 €+07$ & $6.73 E+04$ & $4.23 E+07$ & $6.30 €+04$ & $-1.29 E+05$ & $1.33 E+04$ & $-8.73 E+04$ & $8.96 E+03$ & $1.84 \mathrm{E}+07$ & $2.10 E+05$ & $2.76 E+08$ & $5.02 €+05$ & $2.97 \mathrm{E}+08$ & $6.30 \mathrm{E}+05$ & $3.599+07$ & $1.62 E+04$ & $1.56 €+08$ & $2.56 \mathrm{E}+05$ & $1.67 €+07$ & \begin{tabular}{|l|}
$6.70 E+04$ \\
\end{tabular} & $3.05 E+05$ & $3.68 \mathrm{E}+04$ & $1.58 E+05$ & $1.62 €+04$ & $1.41 巨+08$ & $2.53 E+05$ \\
\hline 2000 & $-5.58 E+07$ & $6.39 E+04$ & $4.24 E+07$ & $6.58 E+04$ & $-8.79 E+04$ & $1.13 E+04$ & $-5.94 \mathrm{E}+04$ & $7.65 \mathrm{E}+03$ & $1.84 \mathrm{E}+07$ & $2.28 E+05$ & $2.75+08$ & $4.45 E+05$ & $2.96 \mathrm{E}+08$ & $7.05 E+05$ & $3.58 \mathrm{E}+07$ & $1.38 \mathrm{E}+04$ & $1.56 €+08$ & $2.33 E+05$ & $1.65 \mathrm{E}+07$ & $5.72 E+04$ & $2.04 E+05$ & $3.20 €+04$ & $1.07 E+05$ & $1.38 E+04$ & $1.40 \mathrm{E}+08$ & $2.32 E+05$ \\
\hline
\end{tabular}

\begin{tabular}{|c|c|c|c|c|c|c|c|c|c|c|c|c|c|c|c|c|c|c|c|c|c|c|c|c|}
\hline \multicolumn{25}{|c|}{ Table S57. Land Optimized - Additive } \\
\hline $\begin{array}{l}\text { Cutoff } \\
\text { Year }\end{array}$ & $\begin{array}{l}\text { GHG Shift } \\
(\mathrm{kg} \\
\left.\mathrm{CO}_{2}{ }^{2} / \mathrm{a}\right)\end{array}$ & SD & \begin{tabular}{|l|l} 
Building \\
Energy (kg \\
$\mathrm{CO}_{2} /$ /a)
\end{tabular} & SD & $\begin{array}{l}\text { Land Use } \\
\text { Snitt } \\
\left(\mathrm{m}^{2} / \mathrm{a}\right)\end{array}$ & & \begin{tabular}{|l|} 
Runoff \\
Reduction \\
$m^{3}$ /al) \\
high
\end{tabular} & SD & $\begin{array}{l}\text { Runoff } \\
\text { Reduction } \\
\left(m^{m} / 3\right) \text { - } \\
\text { low }\end{array}$ & SD & $\begin{array}{l}\text { Waste } \\
\text { Uptake } \\
(\mathrm{kg} / \mathrm{a})\end{array}$ & SD & \begin{tabular}{|l} 
Greens \\
Produced \\
(cup eq./a)
\end{tabular} & SD & $\begin{array}{l}\text { Red and } \\
\text { Orange } \\
\text { Produced } \\
\text { (cup eq./a) }\end{array}$ & so & $\begin{array}{l}\text { Other } \\
\text { Produced } \\
\text { (cup eq./a) }\end{array}$ & SD & \begin{tabular}{|l} 
Planted \\
Araea \\
$\left(\mathrm{m}^{2} / \mathrm{a}\right)$
\end{tabular} & SD & \begin{tabular}{|l}
$\begin{array}{l}\text { Roof Area } \\
\left(\mathrm{m}^{2}\right)\end{array}$ \\
\end{tabular} & SD & $\begin{array}{l}\text { Revenue } \\
\text { (USD/a) }\end{array}$ & SD \\
\hline & $-1.67 \mathrm{E}+07$ & $9.73 \mathrm{E}+04$ & $3.17 \mathrm{E}+06$ & $8.20 \mathrm{E}+04$ & $4.44 \mathrm{E}+06$ & $2.31 \mathrm{E}+04$ & $-2.03 \mathrm{E}+06$ & $2.81 \mathrm{E}+04$ & $-1.37 \mathrm{E}+06$ & $1.90 E+04$ & $1.06 \mathrm{E}+07$ & $1.42 E+05$ & \begin{tabular}{|l|l|} 
cipu eq./2) \\
$6.29 \mathrm{E}+06$ \\
\end{tabular} & $9.83 E+02$ & $\begin{array}{l}\text { (c)peq eq/a) } \\
1.08 E+08 \\
\end{array}$ & $7.34 \mathrm{E}+05$ & & $1.42 E+05$ & 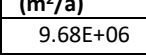 & \begin{tabular}{|c|c|}
50 & $3.43 E+04$ \\
\end{tabular} & \begin{tabular}{|l|l|}
$1 \mathrm{~m})$ & $1.71 \mathrm{E}+06$ \\
\end{tabular} & $3.43 E+04$ & $\frac{105 / \mathrm{a})}{3.13 \mathrm{E}+07}$ & $2.63 \mathrm{E}+05$ \\
\hline 1910 & $-1.51 E+07$ & $5.24 €+04$ & $1.86 \mathrm{E}+06$ & $8.62 E+04$ & $4.12 \mathrm{E}+06$ & $2.16 \mathrm{E}+04$ & $-1.41 E+06$ & $2.89 \mathrm{E}+04$ & $-9.50 \mathrm{E}+05$ & $1.95 E+04$ & $7.48 E+06$ & $1.46 \mathrm{E}+05$ & $6.29 E+06$ & $1.23 \mathrm{E}+03$ & $9.19 E+07$ & $5.22 E+05$ & $7.59 \mathrm{E}+07$ & $1.46 \mathrm{E}+05$ & $8.92 E+06$ & $3.53 \mathrm{E}+04$ & $9.44 \mathrm{E}+05$ & $3.53 \mathrm{E}+04$ & $3.00 £+07$ & $2.15 E+05$ \\
\hline 1920 & $-1.50 \mathrm{C}+\mathbf{7}$ & $5.33 E+04$ & $1.36 E+06$ & $8.44 E+04$ & $4.14 \mathrm{E}+06$ & $2.26 E+04$ & $-1.18 E+06$ & $2.72 E+04$ & $-7.99 E+05$ & $1.845+04$ & $6.35 E+06$ & $1.37 E+05$ & $6.29 E+06$ & $6.25 E+02$ & $8.72 E+07$ & $5.611+05$ & $7.55 E+07$ & $1.07 E+05$ & $8.645+06$ & $3.32 E+04$ & $6.71 E+05$ & $3.32 E+04$ & $3.18 E+07$ & $2.006+05$ \\
\hline & $-1.49 E+07$ & $5.12 E+04$ & $1.04 E+06$ & $8.04 E+04$ & $4.14 \mathrm{E}+06$ & $2.211+04$ & $-1.05 E+06$ & $2.60 E+04$ & $-7.10 E+05$ & $1.76 E+04$ & $5.695+06$ & $1.33 E+05$ & $6.29 E+06$ & $1.87 \mp+02$ & $8.44 E+07$ & $5.38 E+05$ & $7.53 E+07$ & $1.84 E+05$ & $8.48 E+06$ & $3.18 E+04$ & $5.11 E+05$ & $3.188+04$ & $3.28 E+07$ & $2.03 E+05$ \\
\hline 1940 & $-1.49 E+07$ & $4.58 E+04$ & $9.14 E+05$ & $7.70 E+04$ & $4.15 E+06$ & $1.86 E+04$ & $-1.00 E+06$ & $2.60 E+04$ & $-6.77 E+05$ & $1.766+04$ & $5.44 E+06$ & $1.31 E+05$ & $6.29 E+06$ & $2.10 E+02$ & $8.344+07$ & $5.50 E+05$ & $7.52 E+07$ & $1.1996+05$ & $8.422 E+06$ & $3.18 E+04$ & $4.511+05$ & $3.18 E+04$ & $3.32 E+07$ & $2.26 E+05$ \\
\hline 1950 & $-1.49 E+07$ & $4.11 \mathrm{E}+04$ & $8.32 E+05$ & $6.94 E+04$ & $4.15 E+06$ & $1.62 E+04$ & $-9.65 E+05$ & $2.30 \mathrm{E}+04$ & $-6.52 E+05$ & $1.55 E+04$ & $5.25 E+06$ & $1.16 E+05$ & $6.298+06$ & $1.83 E+02$ & $8.26 E+07$ & $5.144+05$ & $7.51 E+07$ & $8.906+04$ & $8.38 E+06$ & $2.805+04$ & $4.05 E+05$ & $2.805+04$ & $3.34 E+07$ & $2.03 E+05$ \\
\hline & $-1.48 E+07$ & $3.47 E+04$ & $6.81 E+05$ & 6.4 & $4.166+06$ & $1.12 E+04$ & $-9.09 E+05$ & $2.11 \mathrm{E}+04$ & $-6.14 \mathrm{E}+05$ & $1.43 E+04$ & $4.97 \mathrm{E}+06$ & $1.07 E+05$ & $6.29 E+06$ & $1.77 E+02$ & $8.15 E+07$ & $4.88 E+05$ & $7.50 \mathrm{E}+07$ & $5.44 E+04$ & $8.31 E+06$ & $2.58 E+04$ & $3.37 E+05$ & $2.58 E+04$ & $3.37 \mathrm{E}+07$ & $2.08 \mathrm{E}+05$ \\
\hline 1970 & $-1.48 E+07$ & 2.2 & +05 & 4.8 & $4.166+06$ & $9.50 \mathrm{E}+03$ & $-8.43 E+05$ & $1.77 E+04$ & $-5.70 \mathrm{E}+05$ & $1.20 E+04$ & $4.64 \mathrm{E}+06$ & $8.94 E+04$ & $6.29 E+06$ & $1.57 \mathrm{E}+02$ & $8.00 E+07$ & $4.25 \mathrm{E}+05$ & $7.49 E+07$ & $4.28 E+04$ & $8.23 E+06$ & $2.16 \mathrm{E}+04$ & $2.57 \mathrm{E}+05$ & $2.16 \mathrm{E}+04$ & $3.41 E+07$ & $1.62 \mathrm{E}+05$ \\
\hline 1980 & $-1.4 \varepsilon$ & 2. & 4.2 & $4.19 E+04$ & +06 & 8.10 & -8.0 & $1.43 E+04$ & $-5.47 E+05$ & $9.64 \mathrm{E}+03$ & $4.46 \mathrm{E}+06$ & $7.20 E+04$ & $6.29 E+06$ & $1.82 E+02$ & $7.92 E+07$ & $3.46 E+05$ & $7.49 E+07$ & $4.64 E+04$ & $8.19 \mathrm{E}+06$ & $1.74 \mathrm{E}+04$ & $2.15 E+05$ & $1.74 \mathrm{E}+04$ & $3.44 E+07$ & $1.59 E+05$ \\
\hline 1990 & $-1.48 E+07$ & $1.78 E+04$ & $3.01 E+05$ & $3.511+04$ & $4.14 E+06$ & $1.50 E+04$ & $-7.60 E+05$ & $1.31 E+04$ & $-5.14 E+05$ & $8.84 \mathrm{E}+03$ & $4.22 E+06$ & $6.60 E+04$ & $6.29 E+06$ & $1.77 E+02$ & $7.80 E+07$ & $3.13 E+05$ & $7.49 \mathrm{E}+07$ & $9.91 E+04$ & $8.13 \mathrm{E}+06$ & $1.60 E+04$ & $1.55 E+05$ & $1.60 \mathrm{E}+04$ & $3.48 E+07$ & $1.511+05$ \\
\hline 2000 & $-1.48 \mathrm{E}+07$ & $1.43 E+04$ & $2.10 E+05$ & $3.11 E+04$ & $4.14 E+06$ & $1.13 \mathrm{E}+04$ & $-7.23 E+00$ & $1.09 E+04$ & $-4.88 E+05$ & $7.34 \mathrm{E}+03$ & $4.03 \mathrm{E}+00$ & $5.48 \mathrm{E}+04$ & $6.28 E+06$ & $1.65 E+04$ & $7.71 E+07$ & $2.21 E+05$ & $7.49 \mathrm{E}+07$ & $8.46 E+04$ & $8.08 E+06$ & $1.33 E+04$ & $1.10 E+05$ & $1.33 E+00$ & $3.51 E+07$ & $1.10 E+05$ \\
\hline
\end{tabular}




\begin{tabular}{|c|c|c|c|c|c|c|c|c|c|c|c|c|c|c|c|c|c|c|c|c|c|c|c|c|}
\hline \multicolumn{25}{|c|}{ Table 558. Land Optimized - Additive, no parking } \\
\hline $\begin{array}{l}\text { Cutoff } \\
\text { Year }\end{array}$ & $\begin{array}{l}\text { GHG Shirt } \\
\text { (Kg }\end{array}$ & SD & $\begin{array}{l}\text { Building } \\
\text { Energy (kg } \\
\text { cooge/a) }\end{array}$ & SD & \begin{tabular}{|l|} 
Land Use \\
shitt \\
$\left(\mathrm{m}^{2} / \mathrm{a}\right)$
\end{tabular} & SD & 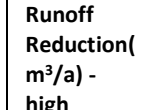 & SD & 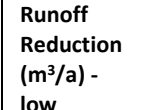 & & $\begin{array}{l}\text { Waste } \\
\text { votake } \\
\text { kt/al }\end{array}$ & & \begin{tabular}{|l} 
Greens \\
Produced \\
lcupea
\end{tabular} & SD & 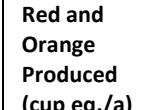 & S5 & $\begin{array}{l}\text { other } \\
\text { Produced } \\
\text { chum eo }\end{array}$ & & \begin{tabular}{|l|} 
Planted \\
Area \\
$\left(m^{2} / 2\right)$
\end{tabular} & SD & 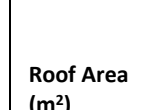 & SD & \begin{tabular}{|l} 
Revenue \\
UusDoal
\end{tabular} & SD \\
\hline 1900 & $\mid-1.67 \mathrm{E}+07$ & $\frac{30.10 E+05}{1.05}$ & $\frac{\mathrm{C}_{2} / \mathrm{da}}{4.44 \mathrm{E}+06}$ & $2.07 \mathrm{E}+04$ & 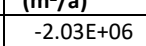 & $3.15 \mathrm{E}+04$ & \begin{tabular}{|l|l|} 
mign & $-1.37 €+06$ \\
\end{tabular} & $2.13 \mathrm{E}+04$ & $6.29 \mathrm{E}+06$ & $1.02 \mathrm{E}+03$ & $1.08 \mathrm{EE}+08$ & $8.17 \mathrm{E}+05$ & 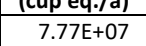 & $1.23 \mathrm{E}+05$ & 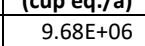 & \begin{tabular}{|l|l|}
30 & $3.84 \mathrm{E}+04$ \\
\end{tabular} & \begin{tabular}{|l|l|} 
(ccup eq./2) \\
$3.13 \mathrm{E}+07$ \\
\end{tabular} & 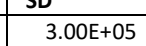 & \begin{tabular}{|l|l|}
$1 m+7 d)$ \\
$1.06 E+07$
\end{tabular} & 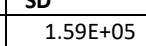 & 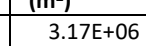 & \begin{tabular}{|l|l|}
$0.28 E+04$ \\
\end{tabular} & \begin{tabular}{|l|l|} 
(USD/a) \\
$1.71 \mathrm{E}+06$ \\
\end{tabular} & $3.84 \mathrm{E}+04$ \\
\hline$\frac{1900}{1910}$ & $-1.51 \mathrm{E}+07$ & $4.476+04$ & $4.12 \mathrm{E}+06$ & $2.22 E+04$ & - $-1.40 \mathrm{EE}+06$ & $2.58 E+04$ & $-9.48 E+05$ & $\frac{1.155+244}{1.75+04}$ & $6.29 \mathrm{EE}+06$ & $1.07+03$ & $\begin{array}{lll}9.18 \mathrm{E}+07 \\
\end{array}$ & $5.096+05$ & $7.596+07$ & $\frac{1.25+105+05}{1.105}$ & $8.91 \mathrm{E}+06$ & $3.154+04+04$ & $2.99 \mathrm{LE}+07$ & $2.29 E+05$ & $7.46 \mathrm{E}+06$ & $\frac{1.53+30 \mathrm{E}+05}{105}$ & $1.86 \mathrm{E}+06$ & 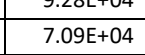 & $9.40 \mathrm{E}+05$ & 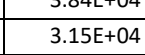 \\
\hline 1920 & $-1.50 \mathrm{E}+07$ & $6.00 E+04$ & $4.14 \mathrm{E}+06$ & $1.80 E+04$ & $-1.19 \mathrm{E}+06$ & $2.90 E+04$ & $-8.01 E+05$ & $1.96 \mathrm{E}+04$ & $6.29 \mathrm{E}+06$ & $5.98 \mathrm{E}+02$ & $8.73 E+07$ & $5.78 \mathrm{E}+05$ & $7.55 E+07$ & $1.16 \mathrm{E}+05$ & $8.65 E+06$ & $3.54 \mathrm{E}+04$ & $3.17 \mathrm{E}+07$ & $1.92 E+05$ & $6.37 \mathrm{E}+06$ & $1.46 E+05$ & $1.37 E+06$ & $8.83 E+04$ & $6.75 E+0$ & $3.54 \mathrm{E}+04$ \\
\hline 1930 & $-1.49 \mathrm{EE}+07$ & $5.04 E+04$ & $4.15 E+06$ & $2.03 E+04$ & $-1.05 E+06$ & $2.62 E+04$ & $-7.096+00$ & $1.776+0$ & $6.29 \varepsilon+00$ & $2.32 E+02$ & $8.44 E+07$ & $5.266+05$ & $7.53 E+07$ & $1.55 E+05$ & $8.48 E+00$ & $3.206+0$ & $3.28 E+07$ & $1.896+0$ & $5.68 \mathrm{E}+0 \mathrm{C}$ & $1.32 E+05$ & $1.04+506$ & $7.896+04$ & $5.09 \varepsilon+00$ & $3.206+04$ \\
\hline 1940 & $-1.49 E+07$ & $4.62 \mathrm{E}+04$ & $4.15 E+06$ & $1.93 E+04$ & $-1.00 E+06$ & $2.47 E+04$ & $-6.77++05$ & $1.67 \mathrm{E}+04$ & $6.29 \mathrm{E}+06$ & $1.73 \mathrm{E}+02$ & $8.34 \mathrm{E}+07$ & $5.22 E+05$ & $7.53 E+07$ & $1.40 E+05$ & $8.42 E+06$ & $3.02 E+04$ & $3.31 E+07$ & $2.03 E+05$ & $5.44 E+06$ & $1.25 E+05$ & $9.24 E+05$ & $7.04 E+04$ & $4.51 E+05$ & $3.02 E+04$ \\
\hline 1950 & $-1.49 \mathrm{E}+07$ & $4.19 \mathrm{E}+04$ & $4.15 E+06$ & $1.78 E+04$ & $-9.62 \mathrm{E}+05$ & $2.56 E+04$ & $-6.50 \mathrm{E}+05$ & $1.73 \mathrm{E}+04$ & $6.29 \mathrm{E}+06$ & $1.80 E+02$ & $8.25 E+07$ & $6.00 E+05$ & $7.51 E+07$ & $7.42 E+04$ & $8.37 \mathrm{~F}+06$ & $3.12 E+04$ & $3.34 E+07$ & $2.20 E+05$ & $5.24 E+06$ & $1.29 E+05$ & $8.20 \mathrm{E}+05$ & $7.34 E+04$ & $4.02 E+05$ & $3.12 E+04$ \\
\hline 1960 & $-1.48 E+07$ & $3.34 \mathrm{E}+04$ & $4.16 E+06$ & $1.31 E+04$ & $-9.06 E+05$ & $2.11 E+04$ & $-6.12 E+05$ & $1.43 \mathrm{E}+04$ & $6.29 \mathrm{E}+06$ & $1.58 \mathrm{E}+02$ & $8.14 \mathrm{E}+07$ & $4.94 E+05$ & $7.50 E+07$ & $6.31 E+04$ & $8.31 \mathrm{E}+06$ & $2.58 \mathrm{E}+04$ & $3.36 \mathrm{E}+07$ & $2.21 E+05$ & $4.95 E+06$ & $1.07 E+05$ & $6.73 \mathrm{E}+05$ & $6.23 \mathrm{E}+04$ & $3.34 E+05$ & $2.58 E+04$ \\
\hline 1970 & $-1.48 E+07$ & $2.88 E+04$ & $4.16 E+06$ & $1.03 E+04$ & $-8.40 E+05$ & $1.95 E+04$ & $-5.58 E+05$ & $1.32 \mathrm{E}+04$ & $6.29 \mathrm{E}+06$ & $1.72 \mathrm{E}+02$ & $7.99 E+07$ & $4.70 E+05$ & $7.50 E+07$ & $5.29 E+04$ & $8.23 E+06$ & $2.39 E+04$ & $3.41 E+07$ & $2.05 E+05$ & $4.62 E+06$ & $9.87 \mathrm{E}+04$ & $5.07 E+05$ & $5.86 \mathrm{E}+04$ & $2.53 E+05$ & $2.39 E+04$ \\
\hline 1980 & $-1.48 E+07$ & $2.19 \mathrm{E}+04$ & $4.15 E+06$ & $9.68 E+03$ & $-8.11 E+05$ & $1.59 E+04$ & $-5.48 E+05$ & $1.08 E+04$ & $6.29 \mathrm{E}+06$ & $1.73 \mathrm{E}+02$ & $7.92 E+07$ & $3.97 E+05$ & $7.49 E+07$ & $5.43 E+04$ & $8.19 E+06$ & $1.95 E+04$ & $3.44 E+07$ & $1.69 E+05$ & $4.47 E+06$ & $8.05 E+04$ & $4.30 \mathrm{E}+05$ & $4.64 E+04$ & $2.17 E+05$ & $1.95 E+04$ \\
\hline 1990 & $-1.48 E+07$ & $1.87 \mathrm{E}+04$ & $4.14 E+06$ & $1.30 E+04$ & $-7.59 E+05$ & $1.32 E+04$ & $-5.13 \mathrm{E}+05$ & $8.90 \mathrm{E}+03$ & $6.29 \mathrm{E}+06$ & $1.56 \mathrm{E}+02$ & $7.80 \mathrm{E}+07$ & $3.27 E+05$ & $7.49 E+07$ & $8.90 E+04$ & $8.13 E+06$ & $1.61 \mathrm{E}+04$ & $3.48 E+07$ & $1.49 \mathrm{E}+05$ & $4.21 \mathrm{E}+06$ & $6.65 \mathrm{E}+04$ & $2.99 E+05$ & $4.00 E+04$ & $1.54 E+05$ & $1.61 \mathrm{E}+04$ \\
\hline 2000 & $-1.48 E+07$ & $1.711+04$ & $4.14 E+06$ & $1.19 E+04$ & $-7.22 E+05$ & $1.02 E+04$ & $-4.88 E+05$ & $6.88 E+03$ & $6.28 E+06$ & $1.73 \mathrm{E}+04$ & $7.71 E+07$ & $2.10 E+05$ & $7.49 E+07$ & $9.36 E+04$ & $8.08 E+06$ & $1.24 \mathrm{E}+04$ & $3.52 E+07$ & $1.06 E+05$ & $4.02 E+06$ & $5.14 \mathrm{E}+04$ & $2.10 \mathrm{E}+05$ & $2.86 \mathrm{E}+04$ & $1.08 E+05$ & $1.24 \mathrm{E}+04$ \\
\hline
\end{tabular}

\begin{tabular}{|c|c|c|c|c|c|c|c|c|c|c|c|c|c|c|c|c|c|c|c|c|c|c|c|c|}
\hline \multicolumn{25}{|c|}{ Table S59. Land Optimized - Subtractive } \\
\hline $\begin{array}{l}\text { Cutoff } \\
\text { vear }\end{array}$ & $\begin{array}{l}\mathrm{GHG} \text { Shift } \\
(\mathrm{kg} h \mathrm{~g} \\
\left.\mathrm{Co}_{2} / \mathrm{a}\right)\end{array}$ & 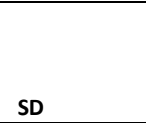 & \begin{tabular}{|l} 
Building \\
Energy (kg \\
Co $_{2} / 2 / \mathrm{ag}$ )
\end{tabular} & SD & $\begin{array}{l}\text { Land Use } \\
\mathrm{shhift}^{\left(\mathrm{m}^{2} / \mathrm{a}\right.}\end{array}$ & SD & \begin{tabular}{|l|} 
Runoff \\
Reduction( \\
$m^{3}$ 'al) \\
high
\end{tabular} & SD & \begin{tabular}{|l|} 
Runoff \\
Reduction \\
$\left(m^{3} / 2\right)-$ \\
low
\end{tabular} & SD & $\begin{array}{l}\text { Waste } \\
\text { Optake } \\
\text { (kkt/a) }\end{array}$ & SD & \begin{tabular}{|l}
$\begin{array}{l}\text { Preens } \\
\text { Produced } \\
\text { (cupe eq./a) }\end{array}$ \\
\end{tabular} & & \begin{tabular}{|l|} 
Red and \\
Orange \\
Produced \\
(cupeq//a)
\end{tabular} & SD & 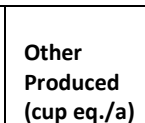 & SD & $\begin{array}{l}\text { 位anted } \\
\text { Area } \\
\text { (m/2/a) }\end{array}$ & & \begin{tabular}{|l|l} 
Roof Area \\
$\left(\mathrm{m}^{2}\right)$
\end{tabular} & SD & $\begin{array}{l}\begin{array}{l}\text { Revenue } \\
\text { (USD/a) }\end{array} \\
\text { (n) }\end{array}$ & SD \\
\hline 1900 & $-2.34 \mathrm{E}+07$ & $3.44 \mathrm{E}+04$ & $2.28 \mathrm{E}+07$ & $7.38 \mathrm{E}+04$ & $-1.40 \mathrm{E}+06$ & $2.66 E+04$ & $-9.48 \mathrm{E}+05$ & $1.79 \mathrm{E}+04$ & $6.29 \mathrm{E}+06$ & $6.79 \mathrm{E}+02$ & $1.10 \mathrm{E}+08$ & $8.72 \mathrm{E}+02$ & \begin{tabular}{|l|l|}
$1.30 E+08$ \\
\end{tabular} & $4.84 \mathrm{E}+04$ & \begin{tabular}{|l|l|l|}
$1.67 \mathrm{E}+07$ \\
\end{tabular} & \begin{tabular}{|l|l|}
$0.40 E+04$ \\
\end{tabular} & \begin{tabular}{|r|}
$1.24 \mathrm{E}+07$ \\
\end{tabular} & $\frac{30.59 E+05}{2.05}-30$ & $1.38 \mathrm{E}+07$ & $1.15 \mathrm{E}+05$ & $3.19 \mathrm{E}+06$ & $7.59 E+04$ & $1.71 \mathrm{E}+06$ & $3.24 \mathrm{E}+04$ \\
\hline 1910 & $-2.38 \mathrm{E}+07$ & $3.91 E+04$ & $2.45 E+07$ & $7.91 \mathrm{E}+04$ & $-7.75 \mathrm{E}+05$ & $2.87 \mathrm{E}+04$ & $-5.23 \mathrm{E}+05$ & $1.94 \mathrm{E}+04$ & $6.29 E+06$ & $1.79 E+02$ & $1.10 \mathrm{E}+08$ & $7.98 \mathrm{E}+02$ & $1.30 \mathrm{E}+08$ & $6.47 \mathrm{E}+04$ & $1.71 \mathrm{E}+07$ & $1.61 \mathrm{E}+04$ & $1.37 \mathrm{E}+07$ & $2.75 \mathrm{E}+05$ & $1.11 \mathrm{E}+07$ & $1.24 E+05$ & $1.86 \mathrm{E}+06$ & $8.44 \mathrm{E}+04$ & $9.45 E+05$ & $3.50 \mathrm{E}+04$ \\
\hline 1920 & $-2.40 E+07$ & $3.87 €+04$ & $2.51 \mathrm{E}+07$ & $6.93 \mathrm{E}+04$ & $-5.53 E+05$ & $2.59 E+04$ & $-3.74 E+05$ & $1.75 E+04$ & $6.29 E+06$ & $1.62 E+02$ & $1.10 E+08$ & $8.84 E+02$ & $1.30 \mathrm{E}+08$ & $5.58 E+04$ & $1.72 E+07$ & $1.59 E+04$ & $1.46 \mathrm{E}+07$ & $2.52 E+05$ & $1.02 E+07$ & $1.111 E+05$ & $1.37 E+06$ & $8.14 \mathrm{E}+04$ & $6.75 E+05$ & $3.16 \mathrm{E}+04$ \\
\hline 1930 & $-2.40 E+07$ & $3.76 E+04$ & $2.55 \mathrm{E}+07$ & $7.03 E+04$ & $-4.19 E+05$ & $2.52 E+04$ & $-2.83 \mathrm{E}+05$ & $1.70 \mathrm{E}+04$ & $6.29 \mathrm{E}+06$ & $1.57 \mathrm{E}+02$ & $1.10 E+08$ & $7.88 \mathrm{E}+02$ & $1.30 \mathrm{E}+08$ & $8.19 E+04$ & $1.73 E+07$ & $1.55 E+04$ & $1.50 E+07$ & $2.61 \mathrm{E}+05$ & $9.65 E+06$ & $1.08 E+05$ & $1.044+06$ & $7.25 E+04$ & $5.111+05$ & $3.08 E+04$ \\
\hline 1940 & $-2.41 E+07$ & $3.33 \mathrm{E}+04$ & $2.56 €+07$ & $7.23 E+04$ & $-3.71 E+05$ & $2.66 \mathrm{E}+04$ & $-2.50 \mathrm{E}+05$ & $1.80 \mathrm{E}+04$ & $6.29 E+06$ & $1.63 \mathrm{E}+02$ & $1.10 E+08$ & $8.79 E+02$ & $1.30 \mathrm{E}+08$ & $7.35 E+04$ & $1.73 \mathrm{E}+07$ & $1.53 \mathrm{E}+04$ & $1.52 E+07$ & $2.25 \mathrm{E}+05$ & $9.45 E+06$ & $1.14 \mathrm{E}+05$ & $9.29 E+05$ & $7.53 €+04$ & $4.52 E+05$ & $3.25 \mathrm{E}+04$ \\
\hline 1950 & $-2.41 E+07$ & $3.65 \mathrm{E}+04$ & $2.57 £+07$ & $6.86 \mathrm{E}+04$ & $-3.30 \mathrm{E}+05$ & $2.55 \mathrm{E}+04$ & $-2.23 \mathrm{E}+00$ & $1.73 \mathrm{E}+04$ & $6.29 E+06$ & $1.50 \mathrm{E}+02$ & $1.10 E+08$ & $8.32 E+02$ & $1.30 \mathrm{E}+08$ & $9.21 E+04$ & $1.74 E+07$ & $1.50 \mathrm{E}+0$ & $1.53 \mathrm{E}+07$ & $2.48 \mathrm{E}+05$ & $9.27 €+06$ & $1.09 E+00$ & $8.25 E+05$ & $8.02 E+04$ & $4.03 E+05$ & $3.12 E+04$ \\
\hline 1960 & $-2.41 E+07$ & $3.22 E+04$ & $2.58 \mathrm{E}+07$ & $5.59 €+04$ & $-2.72 E+05$ & $2.08 E+04$ & $-1.84 E+00$ & $1.40 E+04$ & $6.29 E+06$ & $1.67 \mathrm{E}+02$ & $1.10 \mathrm{E}+08$ & $7.60 \mathrm{E}+02$ & $1.30 E+08$ & $1.09 E+05$ & $1.74 \mathrm{E}+07$ & $1.49 \mathrm{E}+04$ & $1.55 \mathrm{E}+07$ & $1.82 \mathrm{E}+05$ & $9.03 E+00$ & $8.74 E+04$ & $6.68 E+05$ & $6.58 E+04$ & $3.32 E+05$ & $2.54 E+04$ \\
\hline 1970 & $-2.41 E+07$ & $2.47 €+04$ & $2.60 \mathrm{E}+07$ & $4.41 E+04$ & $-2.13 E+05$ & $1.70 €+04$ & $-1.44 E+00$ & $1.15 E+04$ & $6.29 \mathrm{E}+06$ & $1.75 E+02$ & $1.10 \mathrm{E}+08$ & $8.79 \varepsilon+02$ & $1.30 E+08$ & $4.95 E+04$ & $1.74 \mathrm{E}+07$ & $1.16 \mathrm{E}+0$ & $1.58 \mathrm{E}+07$ & $1.56 \mathrm{E}+05$ & $8.78 E+06$ & $7.14 E+04$ & $5.24 \mathrm{E}+05$ & $5.22 \mathrm{5} E+04$ & $2.60 E+05$ & $2.07 E+04$ \\
\hline 1980 & $-2.41 E+07$ & $2.28 E+04$ & $2.61 \mathrm{E}+07$ & $3.57 \mathrm{~F}+04$ & $-1.75 E+05$ & $1.45 E+04$ & $-1.18 E+00$ & $9.79 E+03$ & $6.29 \mathrm{E}+06$ & $1.75 E+02$ & $1.10 \mathrm{E}+08$ & $8.75 E+02$ & $1.30 E+08$ & $5.95 E+04$ & $1.75 E+07$ & $1.09 E+04$ & $1.61 E+07$ & $1.066+05$ & $8.62 E+06$ & $6.06 \mathrm{E}+04$ & $4.23 E+05$ & $4.155+04$ & $2.14 \mathrm{E}+05$ & $1.77 \mathrm{E}+04$ \\
\hline 1990 & $-2.42 E+07$ & $2.06 E+04$ & $2.62 E+07$ & $3.16 \mathrm{E}+04$ & $-1.27 \varepsilon+05$ & $1.29 \mathrm{E}+04$ & $-8.59 \mathrm{E}+04$ & $8.72 \mathrm{E}+03$ & $6.29 \mathrm{E}+06$ & $1.55 \mathrm{E}+02$ & $1.10 \mathrm{E}+08$ & $8.68 \mathrm{E}+02$ & $1.30 \mathrm{E}+08$ & $1.06 E+05$ & $1.75 E+07$ & $1.07 E+04$ & $1.63 \mathrm{E}+07$ & $7.39 \mathrm{E}+04$ & $8.42 E+06$ & $5.44 E+04$ & $2.98 E+05$ & $3.71 \mathrm{E}+04$ & $1.55 \mathrm{E}+05$ & $1.58 \mathrm{E}+04$ \\
\hline 2000 & $-2.42 E+07$ & $1.66 E+04$ & $2.63 E+07$ & $2.75 E+04$ & $-8.95 E+04$ & $1.08 E+04$ & $-6.05 E+04$ & $7.27 \varepsilon+03$ & $6.29 E+06$ & $1.80 E+02$ & $1.10 \mathrm{E}+08$ & $6.83 \mathrm{E}+02$ & $1.30 \mathrm{E}+08$ & $9.02 E+04$ & $1.75 E+07$ & $6.87 \mathrm{6}+03$ & $1.64 E+07$ & $4.17 E+04$ & $8.26 E+06$ & $4.645+04$ & $2.122 \notin+05$ & $3.27 E+04$ & $1.09 E+05$ & $1.311+04$ \\
\hline
\end{tabular}

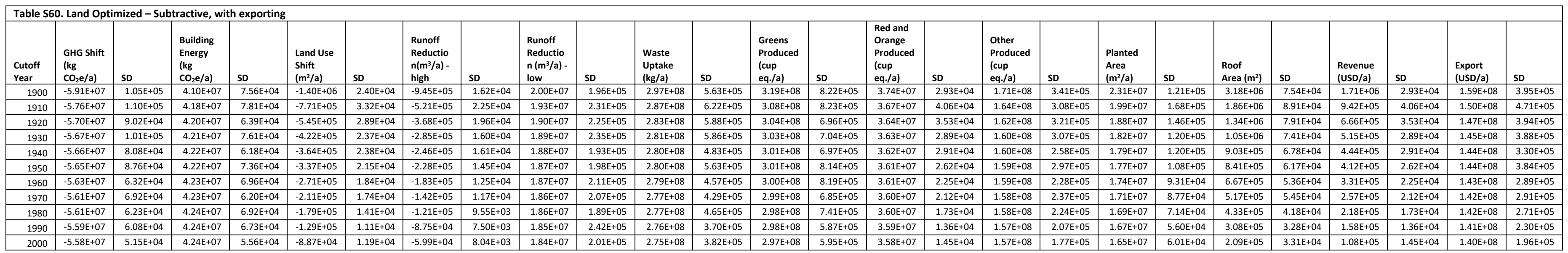




\begin{tabular}{|c|c|c|c|c|c|c|c|c|c|c|c|c|c|c|c|c|c|c|c|c|c|c|c|c|}
\hline \multicolumn{25}{|c|}{ Table S61. Nutrient Demand Optimized - Addi } \\
\hline $\begin{array}{l}\text { Cutoff } \\
\text { vear }\end{array}$ & $\begin{array}{l}\mathrm{GHG} \text { Shift } \\
(\mathrm{kg} \\
\left.\mathrm{Co}_{2} / \mathrm{a} / \mathrm{a}\right)\end{array}$ & SD & 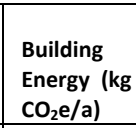 & SD & \begin{tabular}{|l} 
Land Use \\
Shift \\
$\left(\mathrm{m}^{2} / \mathrm{a}\right)$
\end{tabular} & SD & \begin{tabular}{|l|} 
Runoff \\
Reduction( \\
$\mathfrak{m}^{3}$ 'al) \\
high
\end{tabular} & SD & \begin{tabular}{|l|} 
Runofft \\
Reduction \\
(m/3/a)- \\
low
\end{tabular} & SD & $\begin{array}{l}\text { Waste } \\
\text { Uptake } \\
(\mathrm{kg} / \mathrm{a})\end{array}$ & & \begin{tabular}{|l} 
Greens \\
Produced \\
(cup eq./a)
\end{tabular} & & \begin{tabular}{|l|} 
Red and \\
Orange \\
Produced \\
Pcup eq./a) \\
\end{tabular} & SD & \begin{tabular}{|l} 
Other \\
Produced \\
(cup eq./a)
\end{tabular} & SD & \begin{tabular}{|l} 
Planted \\
Araea \\
$\left(\mathrm{m}^{2} / \mathrm{a}\right)$
\end{tabular} & SD & \begin{tabular}{|l} 
Roof Area \\
$\left(\mathrm{m}^{2}\right)$
\end{tabular} & SD & $\begin{array}{l}\begin{array}{l}\text { Revenue } \\
\text { (USD/a) }\end{array} \\
\text { P }\end{array}$ & SD \\
\hline 1900 & 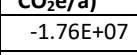 & $1.03 E+05$ & $\begin{aligned} & 2.217 \\
& 3.19 E+06 \\
&\end{aligned}$ & $9.10 \mathrm{E}+04$ & $4.82 \mathrm{E}+06$ & $5.08 E+04$ & \begin{tabular}{|l|l|}
$\operatorname{lign}$ & $-204 \mathrm{E}+06$ \\
\end{tabular} & $2.90 \mathrm{E}+04$ & $-1.38 \mathrm{E}+06$ & $1.96 \mathrm{E}+04$ & $1.07 \mathrm{E}+07$ & $\begin{aligned} 30 \\
1.46 \mathrm{E}+05 \\
\end{aligned}$ & $\begin{aligned} 2.54 E+07 \\
\end{aligned}$ & $1.15 \mathrm{E}+05$ & $\begin{aligned} 8.19 \mathrm{E}+07 \\
\end{aligned}$ & $3.84 \mathrm{E}+05$ & \begin{tabular}{|l} 
\\
$7.81 E+07$ \\
\end{tabular} & $3.73 E+05$ & $9.68 \mathrm{E}+06$ & $\begin{aligned} 30.54 \mathrm{E}+04 \\
\end{aligned}$ & \begin{tabular}{|l}
$1.71 \mathrm{E}+06$ \\
\end{tabular} & $3.54 \mathrm{se+04}$ & $2.85 \mathrm{E}+07$ & $2.71 E+05$ \\
\hline 1910 & $-1.65 E+07$ & $9.41 E+04$ & $1.85 \mathrm{E}+06$ & $8.84 \mathrm{E}+04$ & $5.25 \mathrm{E}+06$ & $5.17 \mathrm{E}+04$ & $-1.40 \mathrm{E}+06$ & $2.73 E+04$ & $-9.48 E+05$ & $1.84 E+04$ & $7.46 \mathrm{E}+06$ & $1.38 E+05$ & $2.36 E+07$ & $1.05 E+05$ & $7.62 E+07$ & $3.32 \mathrm{E}+05$ & $7.27 \varepsilon+07$ & $3.22 E+05$ & $8.911+06$ & $3.33 E+04$ & $9.39 \mathrm{E}+05$ & $3.33 E+04$ & $2.83 E+07$ & $2.32 \mathrm{E}+05$ \\
\hline 1920 & $-1.61 E+07$ & $9.64 \mathrm{E}+04$ & $1.37 \mathrm{~F}+06$ & $8.63 \mathrm{E}+04$ & $5.39 E+06$ & $5.19 \mathrm{E}+04$ & $-1.19 \mathrm{E}+06$ & $2.68 \mathrm{E}+04$ & $-8.02 E+05$ & $1.81 E+04$ & $6.37 \mathrm{E}+06$ & $1.35 E+05$ & $2.30 E+07$ & $1.03 E+05$ & $7.43 \mathrm{E}+07$ & $3.37 \mathrm{E}+05$ & $7.09 E+07$ & $3.08 E+05$ & $8.65 E+06$ & $3.27 \mathrm{E}+04$ & $6.76 \mathrm{E}+05$ & $3.27 E+04$ & $2.81 \mp+07$ & $2.60 \mathrm{E}+05$ \\
\hline 1930 & $-1.57 \mathrm{E}+07$ & $7.86 E+04$ & $1.05 \mathrm{E}+06$ & $7.10 \mathrm{E}+04$ & $5.49 E+06$ & $4.62 E+04$ & $-1.05 E+06$ & $2.51 \mathrm{E}+04$ & $-7.13 \mathrm{E}+05$ & $1.70 E+04$ & $5.70 \mathrm{E}+06$ & $1.27 \mathrm{E}+05$ & $2.26 \mathrm{E}+07$ & $1.08 E+05$ & $7.30 \mathrm{E}+07$ & $3.43 E+05$ & $6.97 \mathrm{E}+07$ & $3.27 \mathrm{E}+05$ & $8.49 E+06$ & $3.06 E+04$ & $5.15 E+05$ & $3.06 \mathrm{E}+04$ & $2.795+07$ & $2.85 \mathrm{E}+05$ \\
\hline 1940 & $-1.56 \mathrm{E}+07$ & $8.37 \mathrm{E}+04$ & $9.13 \mathrm{E}+05$ & $7.21 E+04$ & $5.53 \mathrm{E}+06$ & $5.04 E+04$ & $-1.00 E+06$ & $2.54 \mathrm{E}+04$ & $-6.76 E+05$ & $1.72 E+04$ & $5.43 E+06$ & $1.28 \mathrm{E}+05$ & $2.25 \mathrm{E}+07$ & $9.72 E+04$ & $7.26 \mathrm{E}+07$ & $3.56 E+05$ & $6.92 \mathrm{E}+07$ & $2.90 \mathrm{E}+05$ & $8.42 E+06$ & $3.10 E+04$ & $4.48 E+05$ & $3.10 \mathrm{E}+04$ & $2.78 E+07$ & $2.77 E+05$ \\
\hline & $-1.56 \mathrm{E}+07$ & $7.81 E+04$ & $8.33 E+05$ & $7.10 \mathrm{E}+04$ & $5.57 \mathrm{~F}+06$ & $4.68 \mathrm{E}+04$ & $-9.66 E+05$ & $2.34 E+04$ & $-6.53 \mathrm{E}+05$ & $1.58 E+004$ & $5.26 \mathrm{E}+00$ & $1.18 E+05$ & $2.24 \mathrm{E}+07$ & $8.02 E+04$ & $7.22 E+07$ & $2.87 E+05$ & $6.89 E+07$ & $2.72 \mathrm{E}+05$ & $8.38 E+06$ & $2.86 E+004$ & $4.07 E+05$ & $2.86 \mathrm{E}+04$ & $2.78 E+07$ & $2.37 E+05$ \\
\hline 1960 & $-1.54 \mathrm{E}+07$ & $7.61 \mathrm{E}+04$ & $6.69 \mathrm{E}+05$ & $6.53 \mathrm{E}+04$ & $5.60 \mathrm{E}+06$ & $4.90 \mathrm{E}+04$ & $-9.05 E+05$ & $2.10 E+04$ & $-6.11 \mathrm{E}+05$ & $1.42 E+04$ & $4.95 E+00$ & $1.06 E+05$ & $2.22 E+07$ & $9.80 E+04$ & $7.17 \mathrm{E}+07$ & $3.16 E+05$ & $6.83 \mathrm{E}+07$ & $2.97 \mathrm{E}+05$ & $8.30 E+06$ & $2.57 \mathrm{E}+04$ & $3.32 E+05$ & $2.57 \mathrm{E}+04$ & $2.76 \mathrm{E}+07$ & $2.14 E+05$ \\
\hline 1970 & $-1.53 E+07$ & $6.69 \mathrm{E}+04$ & $5.17 \mathrm{E}+05$ & $5.55 E+04$ & $5.65 \mathrm{E}+06$ & $4.63 E+04$ & $-8.44 E+00$ & $1.99 E+04$ & $-5.770 \mathrm{E}+05$ & $1.34 E+04$ & $4.64 \mathrm{E}+06$ & $1.00 E+05$ & $2.20 \mathrm{E}+07$ & $7.71 E+04$ & $7.11 E+07$ & $3.09 E+05$ & $6.78 E+07$ & $2.66 \mathrm{E}+05$ & $8.23 E+06$ & $2.43 E+04$ & $2.57 \mathrm{E}+05$ & $2.43 E+04$ & $2.74 E+07$ & $2.03 E+05$ \\
\hline 1980 & $-1.52 E+07$ & $5.64 \mathrm{E}+04$ & $4.27 \mathrm{f}+05$ & $4.16 E+04$ & $5.67 \mathrm{E}+06$ & $4.62 E+04$ & $-8.08 E+005$ & $1.46 E+04$ & $-5.46 \mathrm{E}+05$ & $9.86 \mathrm{E}+03$ & $4.46 E+006$ & $7.37 \mathrm{E}+04$ & $2.19 E+07$ & $7.30 E+04$ & $7.07 E+07$ & $2.35 E+05$ & $6.75 E+07$ & $2.26 \mathrm{E}+05$ & $8.19 E+06$ & $1.78 E+04$ & $2.14 \mathrm{E}+05$ & $1.78 \mathrm{E}+04$ & $2.72 E+07$ & $1.70 E+05$ \\
\hline 1990 & $-1.51 E+07$ & $5.06 E+04$ & $2.977+05$ & $3.62 E+04$ & $5.71 E+06$ & $4.60 \mathrm{E}+04$ & $-7.59 E+00$ & $1.32 E+04$ & $-5.13 E+05$ & $8.90 E+03$ & $4.21 E+00$ & $6.65 \mathrm{E}+04$ & $2.17 €+07$ & $6.23 E+04$ & $7.02 E+07$ & $2.01 E+0$ & $6.70 €+07$ & $1.911+05$ & $8.13 \mathrm{E}+06$ & $1.61 \mathrm{E}+04$ & $1.53 \mathrm{E}+0$ & $1.61 E+04$ & $2.71 £+07$ & $1.50 E+05$ \\
\hline 2000 & $-1.50 E+07$ & $4.46 E+04$ & $2.14 \mathrm{E}+05$ & $3.06 \mathrm{E}+04$ & $5.74 E+06$ & $4.19 E+0$ & $-7.23 E+00$ & $1.06 \mathrm{E}+04$ & $-4.89 E+05$ & $7.16 \mathrm{E}+03$ & $4.03 E+00$ & $5.35 E+04$ & $2.16 \mathrm{E}+07$ & $5.60 \mathrm{E}+0$ & $6.99 \mathrm{E}+07$ & $1.80 E+0$ & $6.66 \mathrm{E}+07$ & $1.72 E+05$ & $8.08 E+06$ & $1.29 E+04$ & $1.11 E+05$ & $1.29 E+04$ & $2.70 E+07$ & $1.16 \mathrm{E}+05$ \\
\hline
\end{tabular}

\begin{tabular}{|c|c|c|c|c|c|c|c|c|c|c|c|c|c|c|c|c|c|c|c|c|c|c|c|c|}
\hline \multicolumn{25}{|c|}{ Table 562. Nutrient Demand Optimized - Additive, no parking } \\
\hline $\begin{array}{l}\text { cutoff } \\
\text { Year }\end{array}$ & $\begin{array}{l}\text { GHG Shift } \\
\text { (kg } \\
\text { co } \text { o }_{2} / \text { a) }\end{array}$ & sD & \begin{tabular}{|l|l} 
Building \\
Energy (kg \\
coerala)
\end{tabular} & & $\begin{array}{l}\text { Land Use } \\
\text { Shitit } \\
\left(\mathrm{m}^{2} / \mathrm{a}\right)\end{array}$ & & 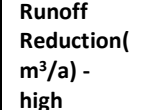 & SD & 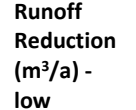 & SD & $\begin{array}{c}\text { Waste } \\
\text { optake } \\
\text { (ktagla) }\end{array}$ & SD & \begin{tabular}{|l} 
Greens \\
Produced \\
scup eq./al)
\end{tabular} & & 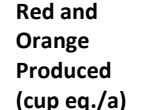 & SD & 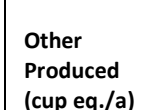 & & 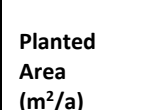 & SD & 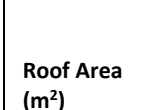 & SD & $\begin{array}{l}\text { Revenue } \\
\text { (USD/a) }\end{array}$ & \\
\hline 1900 & $-1.76 \mathrm{E}+07$ & $9.63 \mathrm{E}+04$ & $4.82 \mathrm{E}+06$ & $5.70 E+04$ & $-2.04 E+06$ & $2.65 \mathrm{E}+04$ & $-1.38 \mathrm{E}+06$ & $1.79 \mathrm{E}+04$ & $2.54 \mathrm{E}+07$ & $1.04 E+05$ & $8.19 \mathrm{E}+07$ & 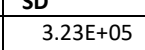 & 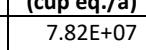 & $3.58 E+05$ & 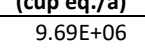 & $3.24 \mathrm{E}+04$ & 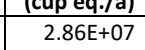 & $2.55 E+05$ & $\frac{\mid(m-7)}{1.07 E+07}$ & $\frac{1.34 \mathrm{E}+05}{105}$ & $3.20 \mathrm{E}+06$ & $8.51 \mathrm{E}+04$ & $\frac{105 / \mathrm{a})}{1.72 \mathrm{E}+06}$ & $3.24 E+04$ \\
\hline 1910 & $-1.65 E+07$ & $9.53 E+04$ & $5.26 E+06$ & $5.05 E+04$ & $-1.411 E+06$ & $2.975+04$ & $-9.51 E+05$ & $2.01 E+04$ & $2.36 \mathrm{E}+07$ & $1.12 E+05$ & $7.63 E+07$ & $3.63 \mathrm{E}+05$ & $7.27 E+07$ & $3.32 E+05$ & $8.92 E+06$ & $3.63 \mathrm{E}+04$ & $2.83 E+07$ & $2.46 E+05$ & $7.48 E+06$ & $1.50 E+05$ & $1.87 \mathrm{E}+06$ & $8.69 \mathrm{E}+04$ & $9.45 E+05$ & $3.63 E+04$ \\
\hline 1920 & $-1.60 \mathrm{E}+07$ & $9.26 E+04$ & $5.40 E+06$ & $5.62 E+04$ & $-1.18 E+06$ & $2.76 E+04$ & $-8.00 E+05$ & $1.87 \mathrm{E}+04$ & $2.30 E+07$ & $1.19 \mathrm{E}+05$ & $7.42 E+07$ & $3.91 \mathrm{E}+05$ & $7.08 E+07$ & $3.40 E+05$ & $8.64 E+06$ & $3.37 \mathrm{E}+04$ & $2.80 \mathrm{E}+07$ & $2.76 E+05$ & $6.36 E+06$ & $1.39 E+05$ & $1.36 E+06$ & $7.89 \mathrm{E}+04$ & $6.73 E+05$ & $3.37 \mathrm{E}+04$ \\
\hline 1930 & $-1.57 \mathrm{E}+07$ & $7.90 E+04$ & $5.50 \mathrm{E}+06$ & $5.17 €+04$ & $-1.05 E+06$ & $2.67 €+04$ & $-7.10 E+05$ & $1.81 \mathrm{E}+04$ & $2.26 \mathrm{E}+07$ & $1.04 \mathrm{E}+05$ & $7.30 E+07$ & $3.50 \mathrm{E}+05$ & $6.96 E+07$ & $3.177+05$ & $8.48 E+06$ & $3.26 E+04$ & $2.79 E+07$ & $2.32 E+05$ & $5.69 \varepsilon+06$ & $1.35 E+05$ & $1.04 E+06$ & $7,7.47+04$ & $5.10 E+05$ & $3.26 E+04$ \\
\hline 1940 & $-1.56 \mathrm{E}+07$ & $8.78 E+04$ & $5.53 \mathrm{E}+06$ & $6.13 \mathrm{E}+04$ & $-9.99 E+05$ & $2.50 €+04$ & $-6.75 E+00$ & $1.69 \mathrm{E}+04$ & $2.24 E+07$ & $1.00 \leftleftarrows+05$ & $7.25 E+07$ & $3.43 E+05$ & $6.92 E+07$ & $3.12 E+05$ & $8.42 E+06$ & $3.05 E+04$ & $2.78 E+07$ & $2.53 E+05$ & $5.42 E+06$ & $1.26 E+05$ & $9.171+05$ & $7.55 E+04$ & $4.477+05$ & $3.05 E+04$ \\
\hline & $-1.55 E+07$ & $9.25 E+04$ & $5.56 \mathrm{E}+06$ & $5.49 \Subset+04$ & $-9.64 €+05$ & $2.58 E+04$ & $-6.51 E+00$ & $1.74 E+04$ & $2.23 \mathrm{E}+07$ & $1.05 E+05$ & $7.22 E+07$ & $3.62 E+05$ & $6.89 \mathrm{E}+07$ & $3.68 E+00$ & $8.38 E+06$ & $3.15 E+04$ & $2.77 \leftleftarrows+07$ & $2.77 €+0$ & $5.25 E+0$ & $1.30 €+00$ & $8.22 E+05$ & $7.57 \boxminus+04$ & $4.04 E+00$ & $3.15 E+04$ \\
\hline & $-1.54 \mathrm{E}+07$ & $6.88 E+04$ & $5.60 E+06$ & $5.15 €+04$ & $-9.05 E+05$ & $1.86 €+04$ & $-6.12 E+00$ & $1.25 \mathrm{E}+04$ & $2.22 E+07$ & $8.72 £+04$ & $7.17 €+07$ & $2.96 \mathrm{E}+05$ & $6.84 \mathrm{E}+07$ & $2.50 €+05$ & $8.30 €+06$ & $2.27 €+04$ & $2.75 E+07$ & $2.25 E+05$ & $4.95 E+06$ & $9.38 €+04$ & $6.75 E+05$ & $5.48 E+04$ & $3.33 E+05$ & $2.27 €+04$ \\
\hline 1970 & $-1.53 E+07$ & $6.33 E+04$ & $5.65 E+06$ & $4.42 \leftleftarrows+04$ & $-8.42 E+05$ & $1.64 \leftleftarrows+04$ & $-5.69 E+00$ & $1.11 E+04$ & $2.20 E+07$ & $8.30 €+04$ & $7.10 E+07$ & $2.67 €+05$ & $6.77 \leftleftarrows+07$ & $2.43 E+05$ & $8.23 E+06$ & $2.00 E+04$ & $2.74 E+07$ & $1.82 E+0$ & 4.63E+06 & $8.26 \mathrm{E}+04$ & $5.13 \mathrm{E}+0$ & $5.01 E+04$ & $2.55 E+00$ & $2.00 E+04$ \\
\hline 1980 & -1.5 & 04 & $5.6 \varepsilon$ & 4.5 & $-8.09 E+05$ & 1.3 & $-5.47 E+0$ & $8.94 E+03$ & +07 & $6.37 £+04$ & $7.07 E+07$ & $2.07 €+05$ & $6.75 E+07$ & $1.92 £+05$ & $8.19 E+06$ & $1.62 \mathrm{E}+04$ & $2.72 E+07$ & $1.57 \mathrm{E}+05$ & $4.46 E+06$ & $6.68 E+04$ & $4.27 E+05$ & $3.87 \mathrm{E}+04$ & $2.15 E+05$ & $1.62 \mathrm{E}+04$ \\
\hline 1990 & $-1.51 E+07$ & $4.90 €+04$ & $5.71 \mathrm{E}+06$ & $3.93 \leftleftarrows+04$ & $-7.61 £+05$ & $1.37 £+04$ & $-5.14 E+0$ & $9.28 \mathrm{E}+03$ & $2.17 €+07$ & $6.20 €+04$ & $7.02 E+07$ & $2.00 E+05$ & $6.70 \mathrm{E}+07$ & $1.91 E+00$ & $8.13 \mathrm{E}+00$ & $1.68 \mathrm{E}+04$ & $2.71 E+07$ & $1.43 E+05$ & $4.22 E+06$ & $6.93 \mathrm{E}+04$ & $3.02 E+05$ & $3.64 \mathrm{E}+04$ & $1.56 \mathrm{E}+0$ & $1.68 E+04$ \\
\hline 2000 & $-1.50 E+07$ & $4.55 €+04$ & $5.75 E+06$ & $3.91 €+04$ & $-7.21 E+00$ & $1.13 \mathrm{E}+04$ & $-4.87 €+05$ & $7.66 \mathrm{E}+03$ & $2.16 \mathrm{E}+07$ & $5.95 €+04$ & $6.98 E+07$ & $1.93 E+05$ & $6.66 \mathrm{E}+07$ & $1.84 €+05$ & $8.08 E+06$ & $1.38 E+04$ & $2.70 \mathrm{E}+07$ & $1.23 E+05$ & $4.02 E+06$ & $5.73 \mathrm{E}+04$ & $2.05 E+0$ & $3.09 E+04$ & $1.07 \notin+05$ & $1.38 E+04$ \\
\hline
\end{tabular}

\begin{tabular}{|c|c|c|c|c|c|c|c|c|c|c|c|c|c|c|c|c|c|c|c|c|c|c|c|c|}
\hline \multicolumn{25}{|c|}{ Table S63. Nutrient Deficit Optimized - Additiv } \\
\hline $\begin{array}{l}\text { Cutoff } \\
\text { Year }\end{array}$ & $\begin{array}{l}\text { GHG Shift } \\
(\mathrm{kg} \\
\mathrm{CO}_{2} / \mathrm{a} / \mathrm{a}\end{array}$ & SD & $\begin{array}{l}\text { Building } \\
\text { Energy (kg } \\
\left.c_{2} / 2 / 2\right)\end{array}$ & SD & $\begin{array}{l}\text { aland Use } \\
\text { Shitt } \\
\left(\mathrm{m}^{2} / \text { a) }\right.\end{array}$ & SD & $\begin{array}{l}\text { Runoff } \\
\text { Reductionl } \\
m^{3} \text { /a) } \\
\text { high }\end{array}$ & SD & $\begin{array}{l}\text { Runoff } \\
\text { Reduction } \\
\left(\mathrm{m}^{3} / \mathrm{a}\right) . \\
\text { low }\end{array}$ & SD & $\begin{array}{l}\text { Waste } \\
\text { Outake } \\
(\mathrm{kg} / \mathrm{a})\end{array}$ & SD & \begin{tabular}{|l} 
Greens \\
Produced \\
(cup eq./a)
\end{tabular} & SD & \begin{tabular}{|l|} 
Red and \\
Orange \\
Produced \\
(cupeq//a)
\end{tabular} & SD & $\begin{array}{l}\text { Oother } \\
\text { Produced } \\
\text { (cup eq./a) }\end{array}$ & SD & \begin{tabular}{|l} 
Planted \\
Araea \\
$\left(\mathrm{m}^{2} / \mathrm{a}\right)$
\end{tabular} & SD & \begin{tabular}{|l|l} 
Roof Area \\
$\left(\mathrm{m}^{2}\right)$
\end{tabular} & SD & $\begin{array}{l}\text { Revenue } \\
\text { (USD/a) }\end{array}$ & SD \\
\hline 1900 & $3.36 \mathrm{E}+06$ & $2.27 \mathrm{~F}+04$ & & $4.33 E+04$ & $-1.36 \mathrm{E}+06$ & $1.91 \mathrm{E}+04$ & $-9.16 E+05$ & $1.29 E+04$ & $1.96 E+07$ & $9.66 \mathrm{E}+02$ & $6.64+07$ & $4.86 \mathrm{E}+02$ & & $1.36 \mathrm{E}+03$ & $5.87 \mathrm{E}+06$ & $1.66 \mathrm{E}+04$ & $1.68 \mathrm{E}+07$ & $2.21 \mathrm{E}+05$ & $6.98 \mathrm{E}+06$ & $8.64+04$ & $2.15 E+06$ & $5.15 E+04$ & $\frac{1.185+06}{1.18 \mathrm{E}+06}$ & $2.31 \mathrm{E}+04$ \\
\hline 1910 & $3.05 E+06$ & $2.766+04$ & $1.02 E+07$ & $3.80 E+04$ & $-9.50 €+05$ & $1.45 E+04$ & $-6.42 E+05$ & $9.78 \mathrm{E}+03$ & $\begin{array}{lll}1.96 E+07 \\
\end{array}$ & $1.22 E+04$ & $6.64 \mathrm{E}+07$ & $2.92 E+04$ & $2.62 E+07$ & $2.95 E+04$ & $5.82 \mathrm{E}+06$ & $1.67 \mathrm{E}+04$ & $2.07 E+07$ & $2.00 E+05$ & $5.06 \mathrm{E}+06$ & $7.17 E+04$ & $1.29 E+06$ & $4.45 E+04$ & $6.66 \mathrm{E}+05$ & $1.88 E+04$ \\
\hline 1920 & $2.95 E+06$ & $2.60 E+04$ & $1.04 E+07$ & $4.30 E+04$ & $-7.92 E+05$ & $1.23 E+04$ & $-5.35 E+05$ & $8.29 E+03$ & $1.96 E+07$ & $3.95 \mathrm{E}+03$ & $6.64 \mathrm{E}+07$ & $1.03 E+04$ & $2.62 E+07$ & $5.36 \mathrm{E}+03$ & $5.79 \mathrm{E}+06$ & $1.61 \mathrm{E}+04$ & $2.21 E+07$ & $2.33 \mathrm{E}+05$ & $4.27 E+06$ & $7.40 \mathrm{E}+04$ & $9.00 E+05$ & $4.60 \mathrm{E}+04$ & $4.54 \mathrm{E}+05$ & $1.99+04$ \\
\hline 1930 & $2.92 E+06$ & $2.75 E+04$ & $1.06 E+07$ & $4.28 E+04$ & $-7.15 E+05$ & $1.58 \mathrm{E}+04$ & $-4.83 E+05$ & $1.07 E+04$ & $1.96 E+07$ & $2.81 E+03$ & $6.64 \mathrm{E}+07$ & $2.56 \mathrm{E}+03$ & $2.62 \leftleftarrows+07$ & $3.37 E+04$ & $5.77 \mathrm{E}+06$ & $1.67 \mathrm{E}+04$ & $2.30 E+07$ & $1.80 \mathrm{E}+05$ & $3.86 E+06$ & $7.69 \mathrm{E}+04$ & $6.89 E+05$ & $4.64 \mathrm{E}+04$ & $3.44 \mathrm{E}+05$ & $2.055+04$ \\
\hline 1940 & $2.90 \mathrm{E}+06$ & $2.25 \mathrm{2}+04$ & $1.06 E+07$ & $3.73 \mathrm{E}+04$ & $-6.88 E+05$ & $1.14 E+04$ & $-4.65 E+05$ & $7.67 E+03$ & $1.96 E+07$ & $1.19 E+04$ & $6.64 E+07$ & $3.09 E+04$ & $2.62 \leftleftarrows+07$ & $2.67 \mathrm{E}+04$ & $5.77 €+06$ & $1.56 \mathrm{E}+04$ & $2.33 E+07$ & $1.59 E+05$ & $3.69 E+06$ & $6.24 \mathrm{E}+04$ & $6.05 E+05$ & $3.97 E+04$ & $2.99 E+05$ & $1.63 \mathrm{E}+04$ \\
\hline 1950 & $2.90 E+06$ & $2.677+04$ & $1.06 E+07$ & & $-6.695+05$ & $1.41 E+04$ & $-4.52 \leftleftarrows+05$ & $9.55 E+03$ & $1.96 E+07$ & $9.25 E+03$ & $6.64 E+07$ & $6.86 \mathrm{E}+03$ & $2.62 \leftleftarrows+07$ & $4.07 E+04$ & $5.77 €+06$ & $1.56 \mathrm{E}+04$ & $2.35 E+07$ & $1.84 E+05$ & $3.59 E+06$ & $7.03 E+04$ & $5.46 E+05$ & $4.66 \mathrm{E}+04$ & $2.71 E+05$ & $1.87 \mathrm{~F}+04$ \\
\hline 1960 & $2.89 E+06$ & $2.077+04$ & $1.07 \mathrm{E}$ & & $-6.39 E+05$ & $1.50 E+04$ & $-4.32 \leftleftarrows+05$ & $1.01 E+04$ & $1.966+07$ & $1.06 E+03$ & $6.64 E+07$ & $1.02 E+03$ & $2.62 \leftleftarrows+07$ & $2.29 E+03$ & $5.77 €+06$ & $1.55 \mathrm{E}+04$ & $2.37 €+07$ & $1.67 €+05$ & $3.44 E+06$ & $7.15 E+04$ & $4.66 E+05$ & $4.34 \mathrm{E}+04$ & $2.33 E+05$ & $1.911+04$ \\
\hline 1970 & $2.88 E+06$ & 2.31 & $1.07 \mathrm{E}$ & & $-6.03 E+05$ & $1.36 E+04$ & $-4.08 E+05$ & $9.19 \mathrm{E}+03$ & $1.966+07$ & $1.02 E+03$ & $6.64 E+07$ & $8.08 E+02$ & $2.62 \leftleftarrows+07$ & $1.42 E+03$ & $5.76 E+06$ & $1.36 \mathrm{E}+04$ & $2.40 E+07$ & $1.78 E+05$ & $3.27 E+06$ & $6.51 \mathrm{E}+04$ & $3.69 E+05$ & $4.25 E+04$ & $1.87 €+05$ & $1.76 E+04$ \\
\hline 1980 & $2.88 \mathrm{E}+06$ & 1.95 & 1.0 & & $-5.83 E+05$ & $1.27 E+04$ & $-3.94 \mathrm{-}+05$ & $8.56 E+03$ & $1.966+07$ & $1.09 E+03$ & $6.64 E+07$ & $6.30 €+02$ & $2.62 E+07$ & $3.53 \mathrm{E}+03$ & $5.76 \mathrm{E}+06$ & $1.37 \mathrm{~F}+04$ & $2.41 E+07$ & $1.64 \mathrm{E}+0$ & $3.17 €+06$ & $5.97 \mathrm{E}+04$ & $3.18 E+05$ & $3.98 \mathrm{E}+0$ & $1.60 €+05$ & $1.62 \mathrm{E}+04$ \\
\hline 1990 & $2.87 €+06$ & $1.51 E+04$ & $1.08 E+07$ & $3.38 E+04$ & $-5.49 E+05$ & $1.05 E+04$ & $-3.711+05$ & $7.111+03$ & $1.966+07$ & $1.14 \mathrm{E}+03$ & $6.64 E+07$ & $6.08 E+03$ & $2.62 \leftleftarrows+07$ & $2.695+03$ & $6 E+06$ & $1.15 E+04$ & $2.44 E+07$ & $1.46 E+05$ & $3.02 E+06$ & 4.61E +04 & $2.28 E+05$ & $2.95 E+04$ & $1.17 €+05$ & $1.26 \mathrm{E}+04$ \\
\hline 2000 & $2.86 E+06$ & $1.38 E+04$ & $1.09 E+707$ & $3.35 E+04$ & $-5.196+05$ & $9.27 \mathrm{9}+03$ & $-3.511+05$ & $6.26 \mathrm{6E}+00$ & $1.96 E+07$ & $1.13 \mathrm{C}+03$ & $6.64 E+07$ & $4.37 \mathrm{E}+03$ & $2.62 E+07$ & $1.42 E+03$ & $5.766+06$ & 1.19EE+04 & $2.46 E+07$ & $1.34 E+05$ & $2.88 E+06$ & 4.13EE+04 & $1.56 E+05$ & $2.82 E+04$ & $8.11 E+04$ & 1.1.3E +04 \\
\hline
\end{tabular}




\begin{tabular}{|c|c|c|c|c|c|c|c|c|c|c|c|c|c|c|c|c|c|c|c|c|c|c|c|c|}
\hline \multicolumn{25}{|c|}{ Table S64. Nutrient Deficit Optimized - Additive, no parking } \\
\hline $\begin{array}{l}\text { Uutooff } \\
\text { Year }\end{array}$ & $\begin{array}{l}\text { GHG Shift } \\
\mathrm{Kkg}^{2} \\
\left.\mathrm{CO}_{2} \mathrm{e} / \mathrm{a}\right)\end{array}$ & SD & \begin{tabular}{|l|l} 
Bunilding \\
Energy (kg \\
$\mathrm{CO}_{2} \mathrm{e} / \mathrm{a}$ )
\end{tabular} & SD & $\begin{array}{l}\text { Land suse } \\
\text { Shit } \\
\left(\mathrm{m}^{2} / 2\right)\end{array}$ & SD & \begin{tabular}{|l|} 
Runoff \\
Reduction \\
$m^{3} /$ /a) \\
high
\end{tabular} & SD & $\begin{array}{l}\text { Runoff } \\
\text { Reduction } \\
\left(m^{3} / / 2\right)- \\
\text { low } \\
\end{array}$ & SD & $\begin{array}{l}\text { Uoste } \\
\text { Untake } \\
(\mathrm{kg} / \mathrm{a})\end{array}$ & SD & \begin{tabular}{|l} 
的eens \\
Produced \\
(cup eq./2)
\end{tabular} & 30 & $\begin{array}{l}\text { Red and } \\
\text { Orange } \\
\text { Produced } \\
\text { (cup eq./a) }\end{array}$ & 30 & \begin{tabular}{|l} 
Other \\
Produced \\
(cup eq./a)
\end{tabular} & & $\begin{array}{l}\text { Planted } \\
\text { Area } \\
\text { (m } / 2 \text { al }\end{array}$ & SD & $\begin{array}{l}\text { Rooot area } \\
\left(\mathrm{m}^{2}\right)\end{array}$ & SD & $\begin{array}{l}\text { (USD/a) } \\
\text { (USula }\end{array}$ & 0 \\
\hline 1900 & & $4.99 E+04$ & & $7.41 E+04$ & & $1.63 \mathrm{E}+04$ & & $1.10 \mathrm{E}+04$ & & $2.13 \mathrm{E}+03$ & $1.83 \mathrm{E}+08$ & $2.87 \mathrm{E}+03$ & & $1.39++03$ & & $2.85 \mathrm{E}+04$ & & $1.89 \mathrm{E}+05$ & & $7.73 \mathrm{E}+04$ & $1.85 \mathrm{E}+06$ & \begin{tabular}{ll|l}
$4.81 E+04$ \\
\end{tabular} & $1.00 E+06$ & $1.99 \mathrm{E}+04$ \\
\hline 1910 & $-3.94 \mathrm{E}+07$ & & & $7.85 \mathrm{E}+04$ & $-4.46 \mathrm{E}+05$ & $1.73 E+04$ & $-3.02 E+05$ & $1.17 \mathrm{E}+04$ & $5.68 \mathrm{E}+07$ & $1.14 \mathrm{E}+03$ & $1.83 \mathrm{E}+08$ & $4.19 \mathrm{E}+03$ & $1.75 \mathrm{E}+08$ & $7.36 \mathrm{E}+03$ & & $2.44 E+04$ & $4.81 \mathrm{E}+07$ & $1.65 E+05$ & $1.16 E+07$ & $8.12 E+04$ & $1.07 \mathrm{E}+06$ & $5.045+04$ & $5.45 E+05$ & $2.11 \mathrm{E}+04$ \\
\hline 1920 & $-3.93 E+07$ & & & $8.39 \mathrm{E}+04$ & $-3.07 E+05$ & $1.44 E+04$ & $-2.08 E+05$ & $9.75 \mathrm{E}+03$ & $5.68 \mathrm{E}+07$ & $1.03 E+03$ & $1.83 E+08$ & $1.87 \mathrm{E}+03$ & $1.75 E+08$ & & & $2.81 E+04$ & $4.88 \mathrm{E}+07$ & $1.73 E+05$ & $1.09 E+07$ & $6.645+04$ & $7.50 \mathrm{E}+05$ & $4.04 \mathrm{E}+04$ & $3.75 E+05$ & $1.76 \mathrm{~F}+04$ \\
\hline 1930 & $-3.93 \mathrm{E}+07$ & & & & $-2.32 \mathrm{E}+05$ & $1.52 \mathrm{E}+04$ & $-1.57 \mathrm{-1.+05}$ & $1.03 \mathrm{E}+04$ & $5.68 \mathrm{E}+07$ & $1.06 \mathrm{E}+03$ & $1.83 \mathrm{E}+08$ & & & & & $2.64 E+04$ & $4.92 E+77$ & $1.49 E+05$ & $1.06 E+07$ & $7.03 E+04$ & $5.711+05$ & & $2.83 E+05$ & $1.86 \mathrm{1.84}+04$ \\
\hline 1940 & & & & & $-1.996+05$ & $1.47 E+04$ & $\begin{array}{l}-1.35 E+05 \\
\end{array}$ & $9.911+03$ & $5.68 \mathrm{5E}+77$ & $1.122 E+03$ & $1.83 E+08$ & & & & & $2.64 E+04$ & 4.93E +07 & $1.26 E+05$ & $1.04 E+07$ & $6.71 E+04$ & 4.94E +05 & & $2.43 E+05$ & $1.79 E+04$ \\
\hline 1950 & & & & & $-1.81 \mathrm{E}+05$ & $1.60 \mathrm{E}+04$ & & $1.08 \mathrm{E}+04$ & $5.68 \mathrm{E}+07$ & $1.10 \mathrm{E}+03$ & $1.83 \mathrm{E}+08$ & 02 & $1.75 \mathrm{E}+08$ & & & $2.47 E+04$ & 4.94E+07 & $1.74 \mathrm{E}+05$ & $1.03 E+07$ & 7. $.18 \mathrm{E}+04$ & 4.46E+05 & & $2.21 \mathrm{E}+05$ & 1.95E .04 \\
\hline 1960 & & 04 & & & $-1.52 \mathrm{E}+05$ & $1.33 E+04$ & $-1.02 E+05$ & $8.98 \mathrm{E}+03$ & $5.68 \mathrm{E}+07$ & $1.14 \mathrm{E}+03$ & $1.83 E+08$ & +02 & $\begin{array}{l}1.75 E+08 \\
1.75-50\end{array}$ & $1.14 \mathrm{E}+03$ & $2.12 \mathrm{E}+07$ & $2.57 €+04$ & 4.95E+07 & $1.25 E+05$ & $1.02 E+07$ & $6.13 \mathrm{E}+04$ & $3.68 E+05$ & $3.89 \mathrm{E}+04$ & $1.85 \leftarrow+05$ & 1.62E 1.04 \\
\hline 1970 & & 04 & & & $-1.19 巨+05$ & $\begin{array}{l}1.18 E+04 \\
\end{array}$ & $-8.02 E+04$ & $7.98 \mathrm{E}+03$ & $5.68 \mathrm{E}+07$ & $1.12 E+03$ & $1.83 E+08$ & $\mathrm{DE}+02$ & $\begin{array}{l}1.75 E+08 \\
.175-50\end{array}$ & $1.41 E+03$ & t07 & $2.50 \mathrm{E}+04$ & 4.97F+07 & $1.57 €+05$ & $1.01 E+07$ & $5.44 \mathrm{E}+04$ & $2.85 E+05$ & 3.49E+04 & $1.45 E+05$ & 1. $.44 E+04$ \\
\hline 1980 & -3.4 & 04 & & & $\begin{aligned}-1.03 E+05 \\
\end{aligned}$ & $77 E+03$ & $-6.97 \mathrm{E}+04$ & $5.93 E+03$ & $8 \mathrm{EE}+07$ & $1.04 E+03$ & $1.83 E+08$ & +02 & 1.75E+08 & $1.36 E+03$ & t07 & $2.59 \mathrm{C}+04$ & $\begin{array}{l}4.97 \mathrm{E}+07 \\
.900\end{array}$ & $1.31 \mathrm{1}+05$ & $9.98 E+06$ & 4.04E+04 & $\begin{array}{l}2.46 E+05 \\
1.70505\end{array}$ & 2.50Е+04 & $1.266+05$ & $\begin{array}{l}1.07 E+04 \\
1.050\end{array}$ \\
\hline 1990 & $\begin{array}{l}-3.933+07 \\
-302520.07\end{array}$ & $5.19 \mathrm{E}+04$ & $\begin{array}{c}1.546+07 \\
155 E+07\end{array}$ & $6.96 \mathrm{6}+04$ & $\frac{-7.66 \mathrm{E}+04}{5.525 \mathrm{C}}$ & $8.50 \mathrm{E}+03$ & $-5.18 E+04$ & $5.74 E+03$ & $5.68 \mathrm{5}+07$ & $1.09 E+03$ & $1.83 E+08$ & $5.21 \mathrm{E}+02$ & $\begin{array}{c}1.75 E+08 \\
1.75-50\end{array}$ & $1.21 E+03$ & $2.122+07$ & $2.06 E+04$ & $\begin{array}{l}4.98 E+07 \\
-500107\end{array}$ & $1.33 E+05$ & $9.86 \mathrm{~g}+06$ & $\begin{array}{l}3.77 E+04 \\
7.50-5\end{array}$ & $1.78 E+05$ & $2.46 \mathrm{EE}+04$ & $9.35 \mathrm{E}+04$ & $1.04 E+04$ \\
\hline 2000 & $-3.93 E+07$ & 4.08E+04 & $1.54 E+07$ & $7.82 E+04$ & $-5.35 \mathrm{E}+04$ & 7.96E+03 & $-3.62 \mathrm{E}+04$ & $5.38 E+03$ & $5.68 E+07$ & $1.09 \mathrm{E}+03$ & $1.83 E+08$ & $4.80 E+02$ & $1.75 E+08$ & 1.42E+03 & $2.12 E+07$ & $2.04 E+04$ & $5.00 E+07$ & 1.144E+00 & $9.76 \mathrm{E}+06$ & 3.69E +04 & $1.26 E+05$ & 2.32E +04 & $6.53 \mathrm{E}+04$ & $9.72 E+03$ \\
\hline
\end{tabular}

\begin{tabular}{|c|c|c|c|c|c|c|c|c|c|c|c|c|c|c|c|c|c|c|c|c|c|c|c|c|c|c|}
\hline \multicolumn{27}{|c|}{ Table 565. Nutrition Optimized - Subtractive, with exporting } \\
\hline $\begin{array}{l}\text { Cutoff } \\
\text { Year }\end{array}$ & 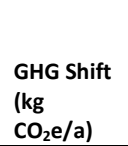 & & $\begin{array}{l}\text { Building } \\
\text { Energy } \\
\text { (kg } \\
\text { Co }_{2} / \text { ala) }\end{array}$ & & $\begin{array}{l}\text { Land Use } \\
\text { shitt } \\
\left(\mathrm{m}^{2} / \mathrm{a}\right)\end{array}$ & & \begin{tabular}{|l|l} 
Runoff \\
Reductio \\
$n\left(m^{3} /\right.$ al) \\
high
\end{tabular} & & $\begin{array}{l}\text { Runoff } \\
\text { Reductio } \\
n\left(m^{3} / a\right)- \\
\text { low }\end{array}$ & SD & $\begin{array}{l}\text { Waste } \\
\text { Uptake } \\
(\mathrm{kg} / \mathrm{a})\end{array}$ & & \begin{tabular}{|l} 
Greens \\
Produced \\
(cup \\
eq./a)
\end{tabular} & & $\begin{array}{l}\text { Red and } \\
\text { orange } \\
\text { Produced } \\
\text { (cup } \\
\text { eq./a) }\end{array}$ & & \begin{tabular}{|l|} 
Other \\
Produced \\
(cup \\
eq./a) \\
\end{tabular} & & $\begin{array}{l}\text { Planted } \\
\text { Area } \\
\left(\mathrm{m}^{2} / \mathrm{a}\right)\end{array}$ & & $\begin{array}{l}\text { Roof } \\
\text { Area }\left(\mathrm{m}^{2}\right)\end{array}$ & & $\begin{array}{l}\text { Revenue } \\
\text { (USD/a) }\end{array}$ & & USD/a) & \\
\hline 1900 & $-3.93 E+07$ & $4.99 \mathrm{E}+04$ & $1.43++07$ & $7.41 E+04$ & $-8.21 \mathrm{E}+05$ & $1.63 E+04$ & $-5.55 E+05$ & $1.10 \mathrm{E}+04$ & $5.68 \mathrm{E}+07$ & $2.13 \mathrm{E}+03$ & $1.83 \mathrm{E}+08$ & \begin{tabular}{|l|l|}
$2.87 \mathrm{E}+03$ \\
\end{tabular} & $1.75 \mathrm{E}+08$ & $1.39 \mathrm{E}+03$ & $2.14 E+07$ & $2.85 \mathrm{E}+04$ & $4.60 \mathrm{E}+07$ & $1.89 \mathrm{E}+05$ & $1.33 \mathrm{E}+07$ & \begin{tabular}{|l|l|}
$7.73 E+04$ \\
\end{tabular} & $1.85 \mathrm{E}+06$ & $4.81 E+04$ & $1.00 \mathrm{E}+06$ & $1.99 E+04$ & $-3.936+07$ & $4.99 \mathrm{E}+04$ \\
\hline 1910 & $-3.944+07$ & $5.29 E+04$ & $1.48 E+07$ & $7.85 E+04$ & $-4.46 E+05$ & $1.73 E+04$ & $-3.02 E+05$ & & $5.68 \mathrm{E}+07$ & $1.14 \mathrm{E}+03$ & $1.83 \mathrm{E}+08$ & & $1.75 €+08$ & $7.36 E+03$ & $2.13 \mathrm{E}+07$ & $2.44 \mathrm{E}+04$ & $4.81 \mathrm{E}+07$ & $1.65 \mathrm{E}+05$ & $1.16 \mathrm{E}+07$ & & $1.07 E+06$ & & $5.45 E+05$ & & & $5.29 \mathrm{E}+04$ \\
\hline 1920 & -3.93 & & & & & & & & 5.68 & & 1.83 & & & & & & & & & & & & & & & $5.33 E+04$ \\
\hline 1930 & -3.93 & & & & & & & & 5.68 & & 1.83 & & & & & $2.64 \mathrm{E}+04$ & & & $1.06 E+07$ & & & & & & & \\
\hline 1940 & & & & & & & & & $5.68 E+07$ & & 1.83 & & & & & $2.64 \mathrm{E}+04$ & 4.93 & & $1.04 \mathrm{E}+07$ & & $4.94 \mathrm{E}+05$ & & & & & $4.80 \mathrm{~A}+04$ \\
\hline 1950 & -3.93 & & & & & & & & $5.68 E+07$ & & $1.83 \mathrm{E}+08$ & & & 1.10 & & $2.47 E+04$ & & 1.74 & 1.03 & & $4.46 \mathrm{E}+05$ & & & & & $\begin{array}{l}4.78 E+04 \\
.98\end{array}$ \\
\hline 1960 & -3.93 & & & & & & & & 5.68 & & $1.83 E+08$ & & $1.75 \leftarrow+08$ & 1.14 & & $2.57 E+04$ & 4.95 & \begin{tabular}{|l|l}
1.25 \\
\end{tabular} & 1.02 & & $3.68 \mathrm{E}+05$ & 3.85 & & & & $\begin{array}{l}4.78 E+04 \\
.98\end{array}$ \\
\hline 1970 & & & & & & & & & 5.68 & & $1.83 E+08$ & & & & & 2.50 & 4.97 & \begin{tabular}{|l|l}
1.57 \\
\end{tabular} & & & $2.85 E+05$ & 3.49 & & & & $\begin{array}{l}4.68 E+04 \\
.98\end{array}$ \\
\hline 1980 & & & & & & & & & & & & & & & & & & 1.3 & & & 2.46 & & & & & \\
\hline 1990 & & & & & & & -5.18 & & $5.68 \mathrm{E}$ & & $1.83 \mathrm{E}$ & 5.211 & & 1.21 & & & 4.98 & 1.33 & 9.86 & 3.77 & 1.78 & & & 1.06 & & \\
\hline 2000 & $-3.93 E+07$ & $4.08 E+04$ & $1.54 \mathrm{E}+07$ & $7.82 E+04$ & 5E+04 & $7.966+03$ & $-3.62 \mathrm{E}+04$ & $5.38 E+03$ & $5.68 \mathrm{~s}+00$ & $1.09 E+03$ & $1.83 E+08$ & $4.80 E+02$ & $1.75 E+08$ & $1.42 E+03$ & 2.12E +07 & $2.04 \mathrm{E}+04$ & $5.00 E+07$ & $1.14 \mathrm{E}+05$ & $9.76 \mathrm{~F}+06$ & $3.69 \mathrm{E}+04$ & $1.26 \mathrm{E}+05$ & $2.32 \mathrm{E}+04$ & $6.53 \mathrm{E}+04$ & $9.72 E+03$ & $-3.93 E+077$ & 4.08E+04 \\
\hline
\end{tabular}




\section{Bibliography}

(1) U.S. Department of Health and Human Services and U.S. Department of Agriculture. 2015-2020 Dietary Guidelines for Americans. 8th Edition; 2015.

(2) USDA. Food Availability (Per Capita) Data System http://www.ers.usda.gov/data-products/foodavailability-(per-capita)-data-system/.aspx (accessed Sep 23, 2016).

(3) National Cancer Institute. Usual Dietary Intakes: Food Intakes, U.S. Population, 2007-10 http://epi.grants.cancer.gov/diet/usualintakes/pop/2007-10/ (accessed Nov 1, 2016).

(4) U.S. Department of Transportation; U.S Department of Commerce. 2007 Commodity Flow Survey; 2010.

(5) United Nations Statistics Division. Detailed Structure and Explanatory Notes - ISIC Rev.3 http://unstats.un.org/unsd/cr/registry/regcst.asp?Cl=2 (accessed Aug 21, 2016).

(6) Archer, E.; Hand, G. A.; Blair, S. N. Validity of U.S. Nutritional Surveillance: National Health and Nutrition Examination Survey Caloric Energy Intake Data, 1971-2010. PLoS One 2013, 8 (10).

(7) United States Census Bureau. American Fact Finder http://factfinder.census.gov/faces/nav/jsf/pages/index.xhtml (accessed Jul 20, 2016).

(8) Massachusetts Department of Correction. Quarterly Report on the Status of Prison Overcrowding, First Quarter 2015; 2015.

(9) Goldstein, B.; Hauschild, M.; Fernández, J.; Birkved, M. Testing the environmental performance of urban agriculture as a food supply in northern climates. J. Clean. Prod. 2016, 135, 984-994.

(10) Goldstein, B.; Hauschild, M.; Fernández, J.; Birkved, M. Urban versus conventional agriculture, taxonomy of resource profiles: a review. Agronomy for Sustainable Development. 2016, pp 1-19.

(11) ISO. 14044: Environmental management—Life cycle assessment—Requirements and guidelines. 2006.

(12) IPCC. Summary for Policy Makers. In Climate Change 2014: Impacts, Adaptation and Vulnerability Contributions of the Working Group II to the Fifth Assessment Report; 2014; pp 1-32.

(13) Goedkoop, M.; Heijungs, R.; Huijbregts, M.; Schryver, A. De; Struijs, J.; Zelm, R. Van. ReCiPe 2008. 2009.

(14) Heller, M. C.; Keoleian, G. a. Greenhouse Gas Emission Estimates of U.S. Dietary Choices and Food Loss. J. Ind. Ecol. 2014, 0 (0), n/a-n/a.

(15) Pirog, R.; Andrew, B. Checking the food odometer: Comparing food miles for local versus conventional produce sales to Iowa institutions; Ames, 2003.

(16) United States Department of Agriculture - National Agricultural Statistics Services. Vegetables 2014 Summary; 2015.

(17) United States Department of Agriculture - National Agricultural Statistics Services. Vegetables 2001 Summary; 2002. 
(18) Clark, H. F.; Brabander, D. J.; Erdil, R. M. Sources, sinks, and exposure pathways of lead in urban garden soil. J. Environ. Qual. 2006, 35 (6), 2066-2074.

(19) City of Boston. BostonMaps: Open Data http://bostonopendata.boston.opendata.arcgis.com/ (accessed Jun 20, 2016).

(20) Government of Massachusetts. MassGIS Data - Land Use (2005) http://www.mass.gov/anf/research-andtech/it-serv-and-support/application-serv/office-of-geographic-informationmassgis/datalayers/lus2005.html (accessed May 30, 2016).

(21) Government of Massachusetts. MassGIS Data - LiDAR Terrain Data http://www.mass.gov/anf/researchand-tech/it-serv-and-support/application-serv/office-of-geographic-informationmassgis/datalayers/lidar.html (accessed Jul 11, 2016).

(22) Government of Massachusetts. MassGIS Data - Protected and Recreational OpenSpace http://www.mass.gov/anf/research-and-tech/it-serv-and-support/application-serv/office-of-geographicinformation-massgis/datalayers/osp.html (accessed Jul 10, 2016).

(23) Davila, C.; Reinhart, C.; Bemis, J. Modeling Boston: A workflow for the generation of complete urban building energy demand models from existing urban geospatial datasets; Cambridge, US, 2016.

(24) City of Boston. Property Assessment 2014 https://data.cityofboston.gov/dataset/Property-Assessment2014/qz7u-kb7x/data (accessed Sep 3, 2016).

(25) U.S. Energy Information Administration. Commercial buildings energy consumption survey http://www.eia.gov/consumption/commercial/ (accessed Aug 4, 2016).

(26) Forman, R. Urban Ecology: Science of Cities; Cambridge University Press: Cambridge, UK, 2014.

(27) Boston Sewer and Water Commission. Combined sewer systems \& outfall maps http://www.bwsc.org/about_bwsc/systems/outfall_maps/outfall_maps.asp (accessed Aug 2, 2016).

(28) Uhl, M.; Schiedt, L. Green Roof Storm Water Retention -Monitoring Results. In 11th International Conference on Urban Drainage; Edinburgh, 2008.

(29) Morgan, S.; Celik, S.; Retzlaff, W. Green Roof Storm-Water Runoff Quantity and Quality. J. Environ. Eng. 2013, 139 (2), 471-478.

(30) Boston Sewer and Water Commission. Monthly Rainfall http://bit.ly/1SDXTxH (accessed Aug 27, 2015).

(31) Boldrin, A.; Christensen, T.; Korner, I.; Krogmann, U. Composting: Mass Balances and Product Quality. In Solid Waste Technology and Management, Volume 1 \& 2; Christensen, T., Ed.; John Wiley \& Sons: Chichester, UK, 2010.

(32) U.S. Energy Information Administration. Residential energy consumption survey https://www.eia.gov/consumption/residential/ (accessed Aug 4, 2016).

(33) U.S. Energy Information Administration. Household energy use in Massachusetts - A closer look at residential energy consumption; 2014.

(34) La Roche, P.; Berardi, U. Comfort and energy savings with active green roofs. Energy Build. 2014, 82, 492-504. 
(35) City of Boston. Boston Community Greenhouse Gas Inventories; 2013.

(36) Centers for Disease Control and Prevention. About the National Health and Nutrition Examination Survey http://www.cdc.gov/nchs/nhanes/about_nhanes.htm.

(37) U.S. Bureau of Labor Statistics. Average Retail Food and Energy Prices, U.S. city average and Midwest Region http://www.bls.gov/regions/mid-

atlantic/data/AverageRetailFoodAndEnergyPrices_USandMidwest_Table.htm (accessed Sep 30, 2016).

(38) United States Department of Agriculture - Agricultural Marketing Service. National Retail Report Specialty Crops: Volume X - Number 40; 2016. 